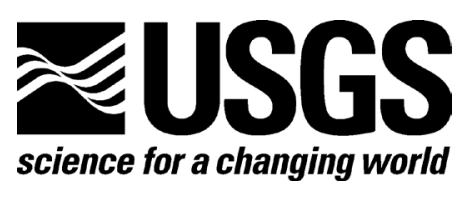

\title{
HAWAIIAN VOLCANO OBSERVATORY 1976 Annual Administrative Report
}

INTRODUCTORY NOTE BY THOMAS L. WRIGHT AND JENNIFER S. NAKATA

COMPILED BY JENNIFER S. NAKATA

SUMMARY 76

JANUARY TO DECEMBER 1976

By Robert Y. Koyanagi, Karen Meagher,

Fred W. KLEIN, AND Gary S. Puniwai

CHRONOLOGICAL SUMMARY

BY PETER W. LIPMAN

OPEN-FILE REPORT 2007-1336

U.S. DEPARTMENT OF THE INTERIOR

U.S. GEOLOGICAL SURVEY 


\section{U.S. Department of the Interior DIRK KEMPTHORNE, Secretary \\ U.S. Geological Survey Mark D. Myers, Director}

\section{U.S. Geological Survey, Reston, Virginia 2007}

For product and ordering information:

World Wide Web: http://www.usgs.gov/pubprod

Telephone: 1-888-ASK-USGS

For more information on the USGS - the Federal source for science about the Earth, its natural and living resources, natural hazards, and the environment: World Wide Web: http://www.usgs.gov

Telephone: 1-888-ASK-USGS

Any use of trade, product, or firm names is for descriptive purposes only and does not imply endorsement by the U.S. Government.

Although this report is in the public domain, permission must be secured from the individual copyright owners to reproduce any copyrighted material contained within this report. 


\section{INTRODUCTORY NOTE}

The Hawaiian Volcano Observatory Summaries have been published in the current format since 1956. The Quarterly Summaries (1956 through 1973) and the Annual Summaries (1974 through 1985) were originally published as Administrative Reports. These reports have been compiled and published as U.S. Geological Survey Open-File Reports. The quarterly reports have been combined and published as one annual summary. All the summaries from 1956 to the present are now available as .pdf files at http://www.usgs.gov/pubprod.

The earthquake summary data are presented as a listing of origin time, depth, magnitude, and other location parameters. Network instrumentation, field station sites, and location algorithms are described. Tilt and other deformation data are included until Summary 77, January to December 1977. From 1978, the seismic and deformation data are published separately, due to differing schedules of data reduction.

There are eight quarters - from the fourth quarter of 1959 to the third quarter of 1961 — that were never published. Two of these ( $4^{\text {th }}$ quarter $1959,1^{\text {st }}$ quarter 1960) have now been published, using handwritten notes of Jerry Eaton (HVO seismologist at the time) and his colleagues. The seismic records for the remaining six summaries went back to California in 1961 with Jerry Eaton. Other responsibilities intervened, and the seismic summaries were never prepared.

\section{Chronology}

The following Kìlauea eruption chronology covers the two recent reports and the six missing quarters:

\begin{tabular}{|l|l|l|l|}
\hline Location & Beginning Date & Ending Date & Comment \\
\hline Kìlauea Iki crater (Kîlauea's summit) & $11 / 14 / 1959$ & $12 / 20 / 1959$ & 19 eruptive episodes \\
\hline Kapoho (lower east rift zone) & $1 / 13 / 1960$ & $2 / 18 / 1960$ & 4 eruption stages \\
\hline Halemaumau (Kīlauea's summit) & $2 / 24 / 1961$ & $2 / 24 / 1961$ & $\begin{array}{l}\text { Intermittent activity during } \\
\text { uninterrupted inflation fol- } \\
\text { lowing the 1960 eruption }\end{array}$ \\
\hline Halemaumau (Kīlauea's summit) & $3 / 22 / 1961$ & $3 / 25 / 1961$ & Same as above. \\
\hline Halemaumau (Kīlauea's summit) & $7 / 10 / 1961$ & $7 / 17 / 1961$ & Same as above. \\
\hline Heiheiahulu (middle east rift zone) & $9 / 22 / 1961$ & $9 / 25 / 1961$ & $\begin{array}{l}\text { First historical east rift erup- } \\
\text { tion at this location }\end{array}$ \\
\hline
\end{tabular}

The 1959-1960 eruptions were among two of the most spectacular Kilauea eruptions. The HVO staff was kept busy with acquisition of unusually high quantities of instrumental data and observations of the two sequences, which were separated by less than one month. Even with a year's interval before the beginning of the summit-east rift sequence in 1961, the staff never caught up, and the seismic records were set aside for later study.

A total of 1,672 earthquakes-1,106 for 1960 and 566 for 1961-are part of HVO's cataloged database. The annual listings have been appended to the $1^{\text {st }}$ Quarter Report of 1960 and to the $4^{\text {th }}$ Quarter Report for 1961 . The number of earthquakes is probably low, biased toward the larger magnitudes. The entire HVO catalog, including 1960 and 1961, is accessible from the ANSS CATALOG SEARCH site at http://www.ncedc.org/anss/catalog-search. 


\section{UNITED STATES \\ DEPARTMENT OF THE INTERIOR \\ GEOLOGICAL SURVEY}

HAWAIIAN VOLCANO OBSERVATORY

SUMMARY 76

JANUARY TO DECEMBER 1976

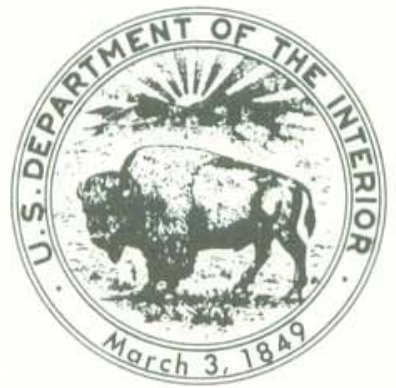

This report is preliminary and has not been edited or reviewed for conformity with

Geological Survey standards and nomenclature

Menlo Park, California 


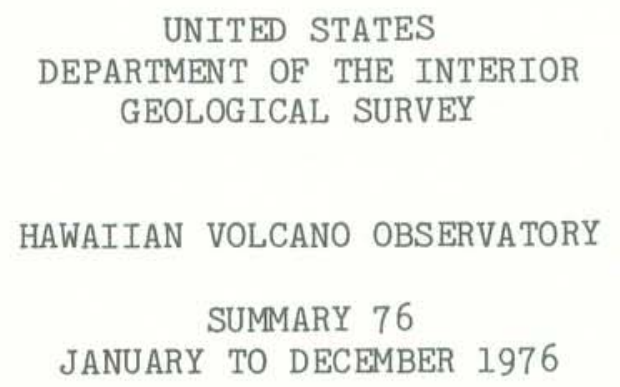

UNITED STATES

DEPARTMENT OF THE INTERIOR

GEOLOGICAL SURVEY

HAWAIIAN VOLCANO OBSERVATORY

SUMMARY 76

JANUARY TO DECEMBER 1976

BY

ROBERT Y. KOYANAGI, KAREN MEAGHER*, FRED W. KLEIN*, AND GARY S. PUNIWAI

CHRONOLOGICAL SUMMARY

BY

PETER W. LIPMAN

OBSERVATORY STAFF

GEOLOGY

GORDON P. EATON (SCIENTIST-IN-CHARGE) REGINALD T. OKAMURA

PETER W. LIPMAN

JOHN P. LOCKWOOD

ROBERT I. TILLING (SCIENTIST-IN-CHARGE)

GEOPHYSICS

LENNART A. ANDERSON

KENNETH T. HONMA

GEORGE KOJIMA

ROBERT Y. KOYANAGI

ERWIN MCPHERSON, JR.

ARNOLD T. OKAMURA

JENNIFER S. NAKATA

GARY S. PUNIWAI
GEOCHEMISTRY
SUPPORT

JOHN C. FORBES

WILLIAM E. FRANCIS

JACK E. HARRIS

MARIE S. ONOUYE

MAURICE K. SAKO

AKIRA YAMAMOTO

KENNETH M. YAMASHITA

*OFFICE OF EARTHQUAKE STUDIES, MENLO PARK, CALIFORNIA 
CONTENTS

Page

Introduction. . . . . . . . 1

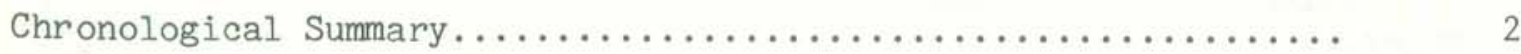

Seismic Instrumentation. ............................

Figure 1 Map of Hawaii showing geographic and geologic

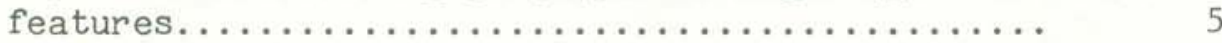

Figure 2 Map of Hawaii showing active seismic stations..... 6

Table 1 Seismic stations on Hawaii operated by the USGS... 7

Table 2 Seismic instrumentation types in use by HVO...... 8

Figure 3 System response curve of the four basic seismograph types in use by HVO............... 9

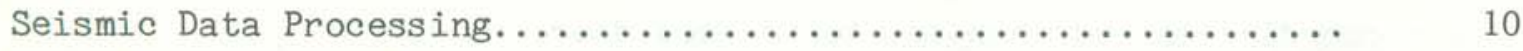

Figure 4 Relationship between duration time and local

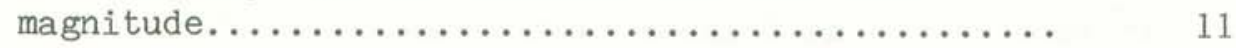

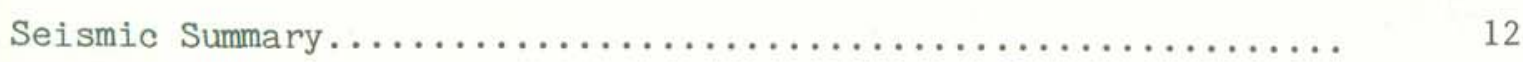

Table 3 Number of earthquakes and minutes of tremor recorded on seismographs around Kilauea..........

Figure 5 Epicenter map of Hawaii showing only magnitudes

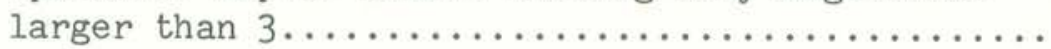

Figure 6 Epicenter map of Hawaii showing all earthquakes...

Figure 7 Epicenter map of the Kilauea/Mauna Loa region showing only magnitudes larger than $3 \ldots \ldots \ldots \ldots$.

Figure 8 Epicenter map of the Kilauea/Mauna Loa region

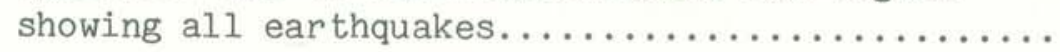

Figure 9 Map of Hawaii showing geographic codes used for classifying earthquakes by region.............

Table 4 Coordinates of named regions used for classifying

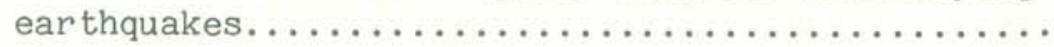

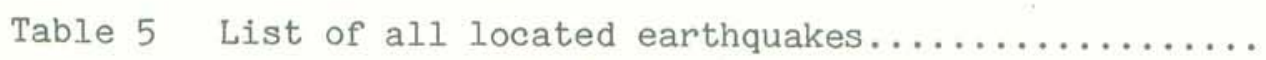


CONTENTS (continued)

$\underline{\text { Page }}$

Table 6 List of located earthquakes magnitude 3.5 and

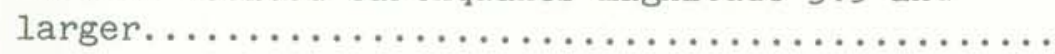

Tilt Instrumentation. . . . . . . . . . 60

Tilting of the ground around Kilauea Caldera............... 61

Table 7 Weekly tilt coordinates at Uwekahuna............ 61

Table 8 Water-tube tilt stations in Hawaii ........... 63

Table 9 Tilt coordinates and changes, December 1975-

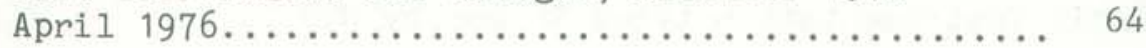

Figure 10 Tilt vectors around Kilauea, December 1975-

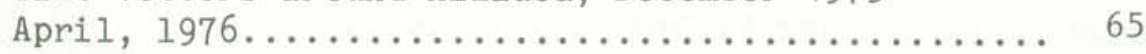

Table 10 Tilt coordinates and changes, April-September,

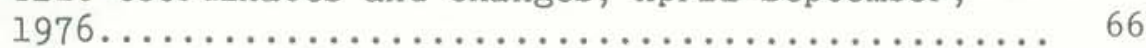

Figure 11 Tilt vectors around Kilauea, April-September, 1976 .

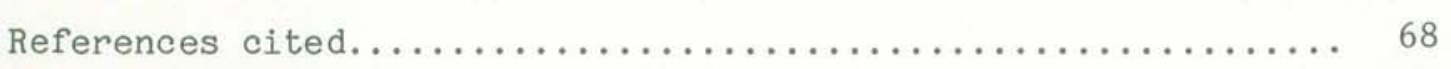




\section{INTRODUCTION}

The Hawaiian Volcano Observatory (HVO) summaries present data gathered during the year together with a chronological narrative intended to describe in geologic terms the volcanic activity associated with the seismic events and tilt data included. The seismic, tilt, and chronological summaries are offered without interpretation as a source of preliminary data. The seismic summary is complete in the sense that all data routinely gathered by the observatory are included. The emphasis in collection of tilt and deformation data has recently shifted from quarterly measurements at a few water-tube tilt stations ("wet" tilt) to a larger number of continuously recording borehole tiltmeters and repeated measurements at numerous spirit-level tilt stations ("dry" tilt). To maintain continuity with past summaries, we will continue to publish weekly data from the Uwekahuna vault tiltmeter (Kilauea summit) and from water-tube tilt stations as they are reoccupied. A comprehensive summary of the numerous and varied tilt and deformation data now gathered is beyond the scope of this publication.

The HVO summaries have been published in various formats since 1956. Summaries prior to 1974 were issued quarterly, but cost, convenience of preparation and distribution, and the large quantities of data dictated an annual format beginning with summary 74 (Koyanagi, et al.) for the year 1974. Summary 74 includes an extensive description of the seismic instrumentation, calibration and processing used in recent years. The present summary includes enough background information on the seismic network and processing to use the data and understand the essentials of how it was gathered.

Publication of the summary represents a group effort by the staffs of the Hawaiian Volcano Observatory and the National Center for Earthquake Research in Menlo Park, California. 


\section{CHRONOLOGICAL SUMMARY}

No eruptive activity occurred at either Kilauea or Mauna Loa during 1976 -- the first such quiet year on the Island of Hawaii since 1966. Perhaps the most

impressive feature recorded at HVO was the continued high seismicity along Kilauea's south flank, reflecting aftershock activity from the November $1975 \mathrm{M}=$ 7.2 earthquake.

The large deflation at the summit of Kilauea related to this earthquake finally bottomed out in February, with a total southeasterly tilt of 241 microradians at Uwekahuna Vault. Extensive Geodimeter and leveling studies of the entire Kilauea network, carried out during the winter and spring, showed that the Kilauea summit area subsided 1.7 meters, and that along the coast between Halape and Keauhou Landing subsidence was as much as 3.5 meters (Tilling et al., 1976). The entire Kilauea area moved seaward in a horizontal component, including areas mauka of the two main rift zones. Preliminary displacement solutions indicate a maximum horizontal displacement vector of 8-9 meters southward at Kalue.

Through the remainder of 1976 the Kilauea summit area showed a distinctive pattern of ground deformation, different from that observed for the past 10 years, that appears to reflect continued adjustment to effects of the 1975 earthquake and related aftershocks. Weak summit inflation--only a few microradians per month--was accompanied by sizable continued seaward horizontal motions of the entire summit area that tended to overwhelm the effect of the inflation. On June 21 and again on July 14 this pattern was interrupted by an abrupt 5-10 microradians of summit deflation accompanied by swarms of sharp earthquakes along the upper east rift zone. Both events were interpreted as marking draining of lava from the summit area into the the east rift zone, with accumulation of lava occurring mainly along upper parts of the rift system. Some connection with lower parts of the east rift zone was also indicated, however, by strikingly increased steaming, centered on the south flank of the prehistoric Heiheihulu cone, that broke out in the spring, and by sizable extensions along geodimeter lines in the same area.

An unusual espisode of large prolonged aseismic deflation at the summit of Kilauea occurred in August and September. Deflation continued at a rate of almost 2 microradians per day, totaling about 40 microradians and finally stopping at about the same level reached during the major subsidence associated with the November, 1975 earthquake. During the deflation episode levels of seismic activity were in no way anomalous, and this deflation episode thus appears unique for the 20-year period for which modern tilt data are available. Previous deflations of similar magnitude have been associated either with a flank eruption or with pronounced local seismicity that marked the locus of shallow intrusive activity. At year's end Kilauea was again in a pattern of steady slow inflation.

Mauna Loa was characterized throughout 1976 by continued summit inflation, accompanied by a surprisingly low level of seismic activity. Earthquake frequency, which was at a high level for 14 months before the July 1975 summit eruption, decreased to near-background levels by late fall 1975 and fluctuated little during the following year. Dry-tilt and Geodimeter measurements showed, however, that the summit area had begun to reinflate immediately after the 1975 eruption, and inflation continued at a high rate through 1976. Tilt 
occurred at a rate of 3-5 microradians per month, and Geodimeter lines across Mokuaweoweo extended by as much at $15 \mathrm{~mm} / \mathrm{month}$. Measurements at Mauna Loa's summit for the time interval August-December 1976 suggested a slight slowing in the rate of inflation, but the changes were too small to be interpreted with confidence. Much HVO staff effort during 1976 was directed to increasing the geodetic baseline control for Mauna Loa, especially Geodimeter and drytilt nets.

Reoccupation for the first time of the "super triangle" Geodimeter lines between Mauna Loa, Mauna Kea, and Hualalai, showed an outward directed displacement vector for the north rim of Mokuaweoweo consistent with summit inflation, and permitted for first time tying to a stable base line the geometry of summit inflation. Both these results and the dry-tilt data demonstrated that the center of Mauna Loa inflation is asymmetric with respect to Mokuaweoweo caldera, lying near the southeast margin of the caldera. This asymmetry is strikingly similar to that long and well documented at Kilauea.

The continued summit inflation of Mauna Loa, as well as the historic record for this volcano in which flank eruptions have typically followed summit activity within a few years, led to the forecast by HV of a probable Mauna Loa rift eruption within three years after the July 1975 summit activity (Lockwood, et al., 1976). Considerable staff activity was directed toward briefing civil defense and other state and local government officials about contingency planning, should Mauna Loa erupt along its northeast rift and threaten Hilo. Additional planning and several field exercises were devoted to enhancing techniques of lava diversion. At the end of the year the HVO staff was becoming increasingly uncertain about the Mauna Loa prediction, however, because of the continued seismic lull as well as the obvious fact that the 25-year period of inactivity at Mauna Loa between 1950 and 1975 was itself atypical in terms of the historic record for this volcano. One obvious major need, for both Mauna Loa and Kilauea, is the reliable geologic understanding of recurrence intervals for different types of activity, that would extend our frame of reference back beyond the 150 years of written history for these volcanoes. 


\section{SEISMIC INSTRUMENTATION}

The network. The Hawaiian Volcano Observatory has installed and maintains an extensive telemetering seismometer network on the island of Hawaii. In January 1976 the seismometer network consisted of 40 stations spread over an area with a diameter of 125 kilometres on the island of Hawaii (Figs. 1 and 2). Of these 40 stations, two are low-gain multicomponent stations (optical), six are two-component, three are three-component, and twenty-nine are vertical only. The coverage is most complete on and around the main center of seismic and volcanic activity, Kilauea Volcano. Other stations in the network are part of a larger net located on other volcanoes of the island of Hawaii. With the exception of HIL, all seismometer signals from the short period network are telemetered to the observatory for recording. During 1976, station DAN on the southeast rift of Mauna Loa was added, and station SCA was moved a short distance and renamed WIL.

Figure 1 is a map of selected geographic and geologic features, and Figure 2 shows the seismic stations which were operated or added during the year. Table 1 lists all seismic stations operated by the U.S. Geological Survey in Hawaii during 1976. Listed are station name, three letter code, coordinates in degrees and minutes, elevation in meters, and other data described below.

Instrumentation and recording. Each telemetering station has a voltage controlled oscillator (VCO) for FM multiplex transmission to HVO via either hardwire or VHF radio. The VCO frequencies are listed in Table 1. These telemetering stations are now all of Type 1, the NCER standard system used in USGS seismic networks (see Table 2 for details). After discrimination, the analog signals from thirty-two stations are recorded on two Develocorders using $16 \mathrm{~mm}$ microfilm. Beginning in late 1975, FM signals from the telemetering network were recorded directly on one inch magnetic tape. Selected larger events are copied onto condensed library tapes which are currently archived in Menlo Park.

Develocorder records are read on a film viewer with 20x magnification. Arrivals are read to the nearest 0.05 second. The recorded arrival times, amplitudes (where readable), and other key data are routinely sent to N.C.E.R. in Menlo Park for computer processing.

In addition to the standard stations, optical seismographs are maintained at Uwekahuna (HVO), Hilo, Maui, and on Oahu (Kipapa station operated by Honolulu Observatory). The less sensitive short period records are used primarily for $\mathrm{S}$ data and amplitude measurements for magnitude calculations to supplement readings from $16 \mathrm{~mm}$ film. Optical seismographs listed in Table 1 are of four types. Types numbered three and four are electro-mechanical systems of high and low gain respectively. Hilo and Haleakala are each equipped with two low-gain Wood-Anderson torsion seismographs. Long period Press-Ewing seismographs record in three components in the Uwekahuna vault. The paper (optical) records as well as the $16 \mathrm{~mm}$ develocorder microfilm are archived at HVO.

Seismograph response and calibration. Displacement response curves for the four short-period seismograph types in use are given in Figure 3. Types three and four are electro-mechanical systems recorded on paper records. The Type 1 curve gives the displacement magnifcation of the standard NCER system from ground motion at the seismometer to the seismic trace as seen on a $20 \mathrm{x}$ Develocorder film viewer. The curves plot the unit response which should be multiplied by the factors CAL listed in Table 1 to get the response for an 
individual station. Individual CAL factors for Type 1 seismographs are equal to the peak-to-peak amplitude measured in $\mathrm{mm}$ on the 20x Develocorder viewer of a 10 microvolt $5 \mathrm{~Hz}$ signal introduced to the preamp/VCO in place of the geophone. Calibration is normally done each time a station is visited, and major changes in attenuation or recalibrations are listed in Table 1 along with the dates they took place. Minor changes in CAL factors may not be listed if they are less than $20 \%$.

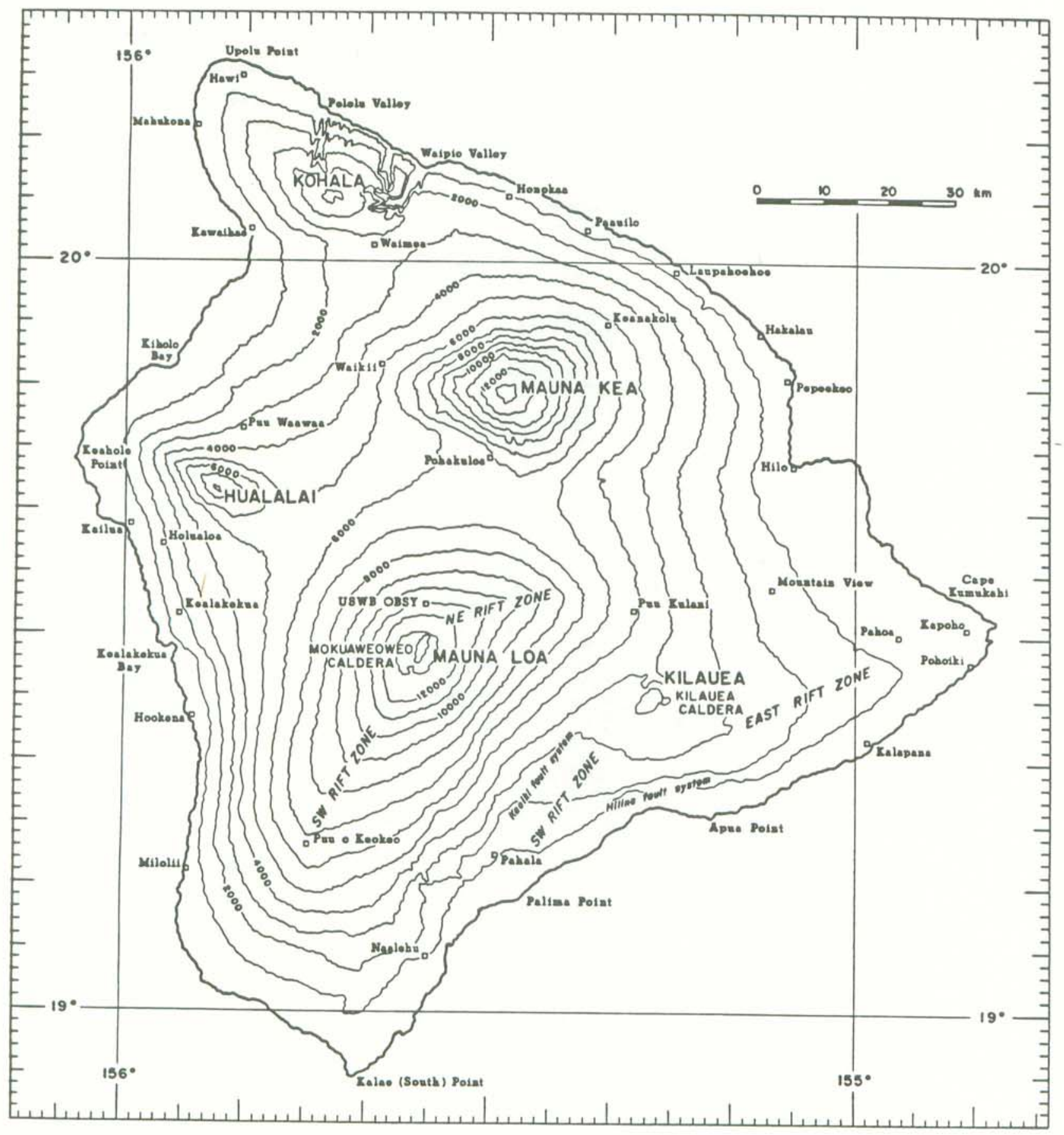

Figure 1 Map of the island of Hawaii showing principal settlements and selected geographic and geologic features. 


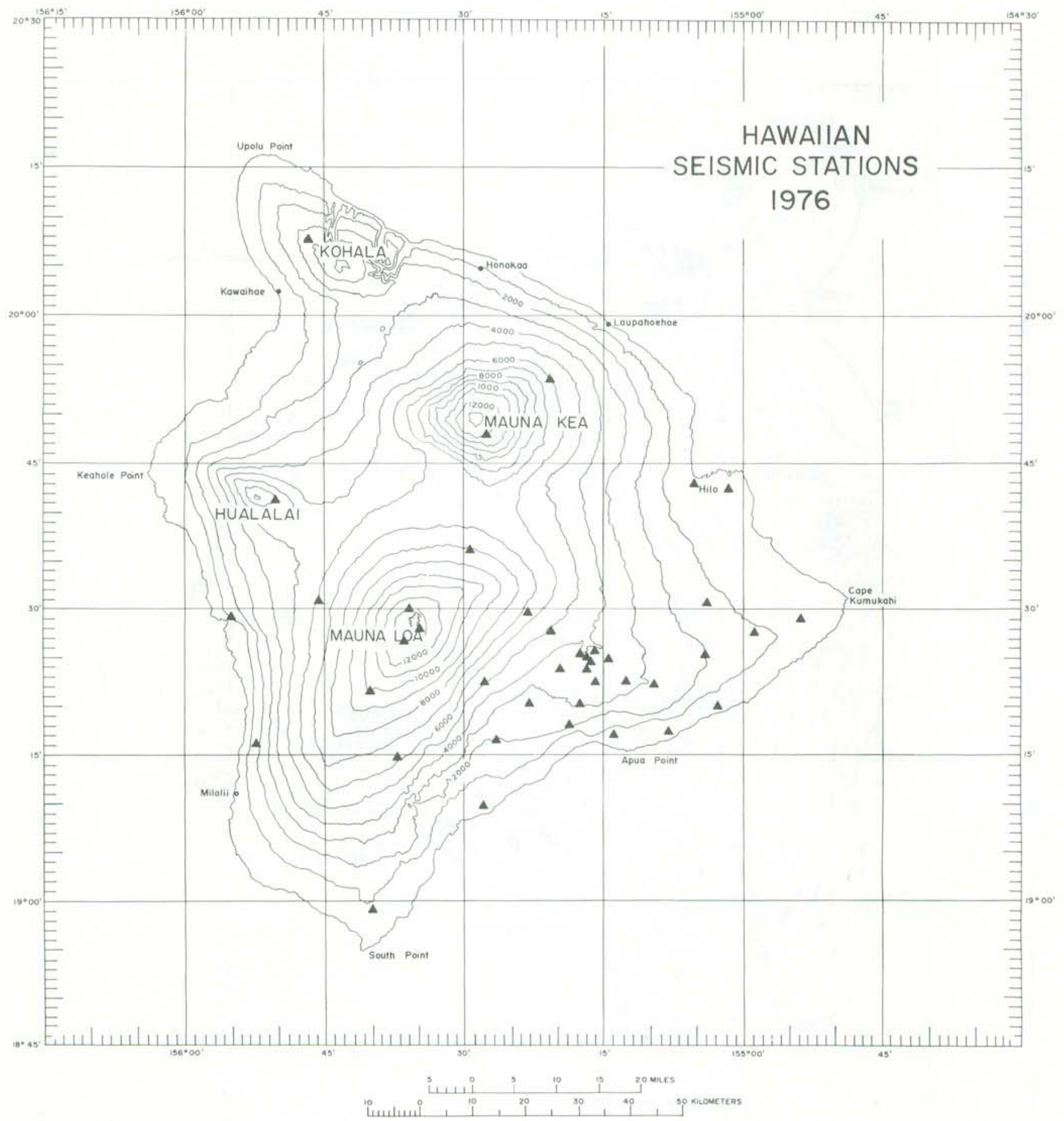

Figure 2 Map of the island of Hawaii showing seismic stations operated by the U.S. Geological Survey. 
TABLE 1.Seismometer stations in Hawaii operated by the U. S. Geological Survey, 1976.

\begin{tabular}{|c|c|c|c|c|c|c|c|c|c|c|c|c|}
\hline Station Name & Code & \multicolumn{2}{|c|}{ LAT-N } & \multicolumn{2}{|c|}{ LON-W } & Delay & ELEV & VCO & $\begin{array}{c}\text { 01d } \\
\text { Type/Cal }\end{array}$ & $\begin{array}{l}\text { Date of } \\
\text { Change }\end{array}$ & \multicolumn{2}{|c|}{$\begin{array}{c}\text { New } \\
\text { Type/Ca1 }\end{array}$} \\
\hline AHUA & $\mathrm{AHU}$ & 19 & 22.40 & 155 & 15.90 & .06 & 1070 & 2380 & 3.8 & $76 / 09 / 29$ & 1 & 4.3 \\
\hline \multirow{3}{*}{ AINAPO } & AIN & 19 & 22.50 & 155 & 27.62 & .33 & 1524 & 1020 & 8.5 & & & \\
\hline & AINE & & & & & & & 2380 & 1 & & & \\
\hline & AINN & & & & & & & 2720 & 1 & & & \\
\hline CAPTAIN COOK & CAC & 19 & 29.29 & 155 & 55.09 & .15 & 323 & 1360 & 3.3 & $76 / 01 / 22$ & 1 & 5.4 \\
\hline \multirow[t]{2}{*}{ CONE PEAK } & $\mathrm{CPK}$ & 19 & 23.70 & 155 & 19.70 & -.04 & 1038 & 1700 & 4.0 & & & \\
\hline & CPKH & & & & & & & 1020 & 1 & & & \\
\hline DANDELION & DAN & 19 & 21.42 & 155 & 40.04 & -.07 & 3003 & 2380 & none & $76 / 05 / 10$ & 1 & 4.8 \\
\hline DESERT & DES & 19 & 20.20 & 155 & 23.30 & -.10 & 815 & 680 & 14.8 & $76 / 03 / 16$ & 1 & 4.2 \\
\hline ESCAPE ROAD & ESR & 19 & 24.68 & 155 & 14.33 & .01 & 1177 & 1360 & 1.7 & $76 / 09 / 21$ & 1 & 2.0 \\
\hline HALE POHAKU & HPU & 19 & 46.85 & 155 & 27.50 & .42 & 3396 & 2720 & 4.5 & $76 / 10 / 02$ & 1 & 4.7 \\
\hline HILINA PALI & HLP & 19 & 17.96 & 155 & 18.63 & .18 & 707 & 2040 & 5.0 & $76 / 01 / 05$ & 1 & 4.0 \\
\hline HUALALAI & HUA & 19 & 41.25 & 155 & 50.32 & .58 & 2189 & 1700 & 2.2 & $76 / 01 / 27$ & 1 & 2.6 \\
\hline \multirow[t]{3}{*}{ HUMUULA } & HSS & 19 & 36.31 & 155 & 29.13 & .35 & 2445 & 1700 & 6.2 & & & \\
\hline & HSSE & & & & & & & 680 & 1 & & & \\
\hline & HSSN & & & & & & & 2720 & 1 & & & \\
\hline KAAPUNA & $\mathrm{KAA}$ & 19 & 15.98 & 155 & 52.28 & .00 & 524 & 1020 & 4.9 & $76 / 12 / 01$ & 1 & 5.2 \\
\hline KAENA & $\mathrm{KAE}$ & 19 & 17.35 & 155 & 7.95 & .15 & 37 & 2380 & 2.2 & $76 / 10 / 27$ & 1 & 1.3 \\
\hline KAHUKU & $\mathrm{KHU}$ & 19 & 14.90 & 155 & 37.10 & .08 & 1939 & 1700 & 3.5 & & & \\
\hline KALALUA & LUA & 19 & 24.55 & 155 & 04.25 & -.02 & 622 & 1020 & 1 & & & \\
\hline \multirow[t]{3}{*}{ KANEKII } & KII & 19 & 30.56 & 155 & 45.90 & .18 & 1841 & 1700 & 7.1 & & & \\
\hline & KIIE & & & & & & & 1020 & 1 & & & \\
\hline & KIIN & & & & & & & 1360 & 1 & & & \\
\hline KAPAPALA RANCH & KPR & 19 & 16.40 & 155 & 26.70 & .05 & 610 & 1700 & 4.33 & $76 / 01 / 26$ & 1 & 6.0 \\
\hline KEANAKOLU & KKU & 19 & 53.39 & 155 & 20.58 & .86 & 1863 & 2380 & 2.3 & & & \\
\hline KIPUKA NENE & KPN & 19 & 20.10 & 155 & 17.40 & .07 & 924 & 1360 & 5.0 & $76 / 08 / 16$ & 1 & 6.4 \\
\hline KOHALA & $\mathrm{KOH}$ & 20 & 7.69 & 155 & 46.77 & .21 & 1166 & 2380 & 12.4 & & & \\
\hline \multirow{2}{*}{ MAUNA LOA } & MLO & 19 & 29.80 & 155 & 23.30 & .24 & 2010 & 1360 & 110.5 & $76 / 01 / 29$ & 1. & 10.0 \\
\hline & $\mathrm{MLOH}$ & & & & & & & 2040 & 1 & & & \\
\hline MAUNA LOA $\mathrm{X}$ & MLX & 19 & 27.60 & 155 & 20.70 & .27 & 1474 & 1360 & 2.1 & & & \\
\hline & MLXH & & & & & & & 2720 & 1 & & & \\
\hline MAKAOPUHI & MPR & 19 & 22.07 & 155 & 9.85 & -.01 & 881 & 2720 & 2.1 & $76 / 01 / 12$ & 1 & 3.3 \\
\hline MOKUAWEOWEO & MOK & 19 & 29.28 & 155 & 35.98 & .28 & 4104 & 2040 & 7.5 & $76 / 09 / 23$ & 1 & 5.4 \\
\hline MOUNTAIN VIEW & MTV & 19 & 30.25 & 155 & 3.75 & .17 & 409 & 680 & 3.2 & $76 / 11 / 18$ & 1 & 7.8 \\
\hline NATIONAL GUARD & NAG & 19 & 42.12 & 155 & 1.72 & .63 & 18 & 1360 & 8.5 & $76 / 02 / 11$ & 1. & 10.2 \\
\hline NORTH PIT & NPT & 19 & 24.90 & 155 & 17.00 & -.06 & 1115 & 680 & 9.0 & $76 / 08 / 17$ & 1 & 4.4 \\
\hline OUTLET & OTL & 19 & 23.40 & 155 & 16.80 & .02 & 1084 & 1360 & 4.15 & $76 / 09 / 22$ & 1 & 4.9 \\
\hline & OTLH & & & & & & & 2040 & 1 & & & \\
\hline PAU & PAU & 19 & 22.62 & 155 & 13.10 & -.06 & 994 & 2040 & 3.8 & & & \\
\hline & PAUH & & & & & & & 1020 & 1 & & & \\
\hline POLIOKEAWE PALI & POL & 19 & 17.02 & 155 & 13.47 & .10 & 169 & 2720 & 4.8 & & & \\
\hline PUU HOHUAULA & PHO & 19 & 28.90 & 154 & 53.40 & .03 & 215 & 2720 & 4.8 & $76 / 01 / 06$ & 1 & 1.9 \\
\hline PUU PILI & PPL & 19 & 9.50 & 155 & 27.87 & .24 & 35 & 1360 & 2.2 & & & \\
\hline RIM & RIM & 19 & 23.90 & 155 & 16.60 & .02 & 1128 & 1020 & 7.3 & & & \\
\hline & RIMH & & & & & & & 2040 & 1 & & & \\
\hline SOUTH POINT & SPT & 18 & 58.91 & 155 & 39.92 & -.07 & 244 & 2040 & 3.6 & & & \\
\hline SOUTHWEST RIFT & SWR & 19 & 27.26 & 155 & 36.30 & .14 & 4048 & 1020 & 12.4 & & & \\
\hline SUMMIT CABIN & SCA & 19 & 28.20 & 155 & 35.08 & .30 & 4048 & 1700 & 111.5 & $76 / 12$ & of: & $f$ \\
\hline TANGERINE & TAN & 19 & 27.79 & 154 & 58.51 & .02 & 351 & 1020 & 1 & $76 / 03 / 17$ & 1 & 8.0 \\
\hline WAHAULA & WHA & 19 & 19.90 & 155 & 2.92 & .06 & 29 & 680 & 3.66 & $76 / 10 / 27$ & 1 & 2.3 \\
\hline WALDRON LEDGE & WLG & 19 & 25.49 & 155 & 15.69 & -.02 & 1067 & 2380 & 12.2 & & & \\
\hline WILKINS & WIL & 19 & 28.15 & 155 & 35.02 & .30 & 4037 & & none & $76 / 12$ & 1 & $5 / 1$ \\
\hline
\end{tabular}


TABLE 1. (continued)

Optical Seismographs

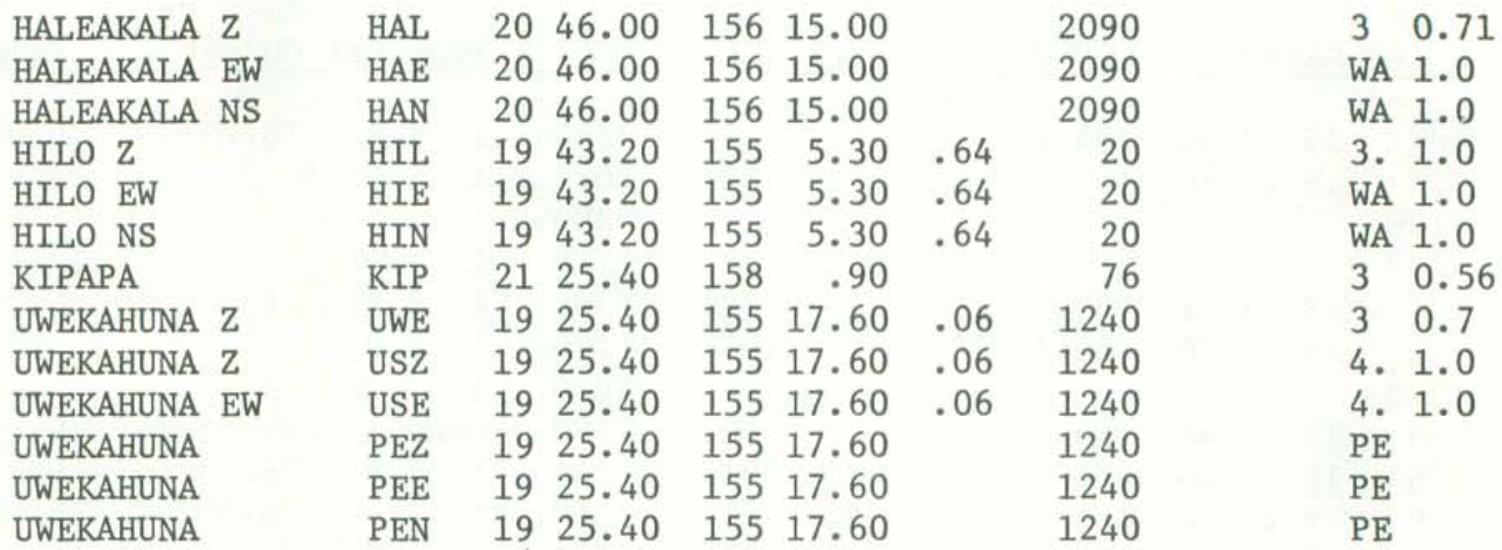

Table 2. -- Seismic Instrumentation Types

Type 1. Consists of:

a) EV-17 - Electrotech EV-17 1.0 sec. period moving magnet vertical component seismometer or horizontal component adjusted for an output of 0.5 volts $/ \mathrm{cm} / \mathrm{sec}$. and 0.8 critically damped.

b) Preamp/VCO

Develco Model 6202 voltage controlled oscillator or a USGS/NCER Model JE202. 3 db points for bandpass filter at $0.1 \mathrm{~Hz}$ and $30 \mathrm{~Hz}$. Signals are transmitted on audio FM carrier over cable or FM radio link to HVO. USGS Model J302 was introducted in 1974.

Type 3. Consists of:

a) EV-17 - Electrotech EV-17 (as described above), Hall-Sears HS-10 0.5 sec. period moving coil seismometer or Observatory-built 0.8 sec. period moving coil seismometer with HVO-built solid state seismic preamplifier (voltage gain, 2000X), or Observatory-built electronmagnetic seismometer approximately 40,000 at $4 \mathrm{~Hz}$.

Type 4. Consists of:

Sprengnether short period vertical and horizontal seismometers (E-W) with $1.5 \mathrm{sec}$ galvanometers, coupling factor $=0.25$, $2 \mathrm{X}$ critically damped. Peak magnitication approximately $1500 \mathrm{X}$ at $2 \mathrm{~Hz}$.

Experimental type amplifier systems are not given type numbers. Type 2 instruments have been discontinued. 


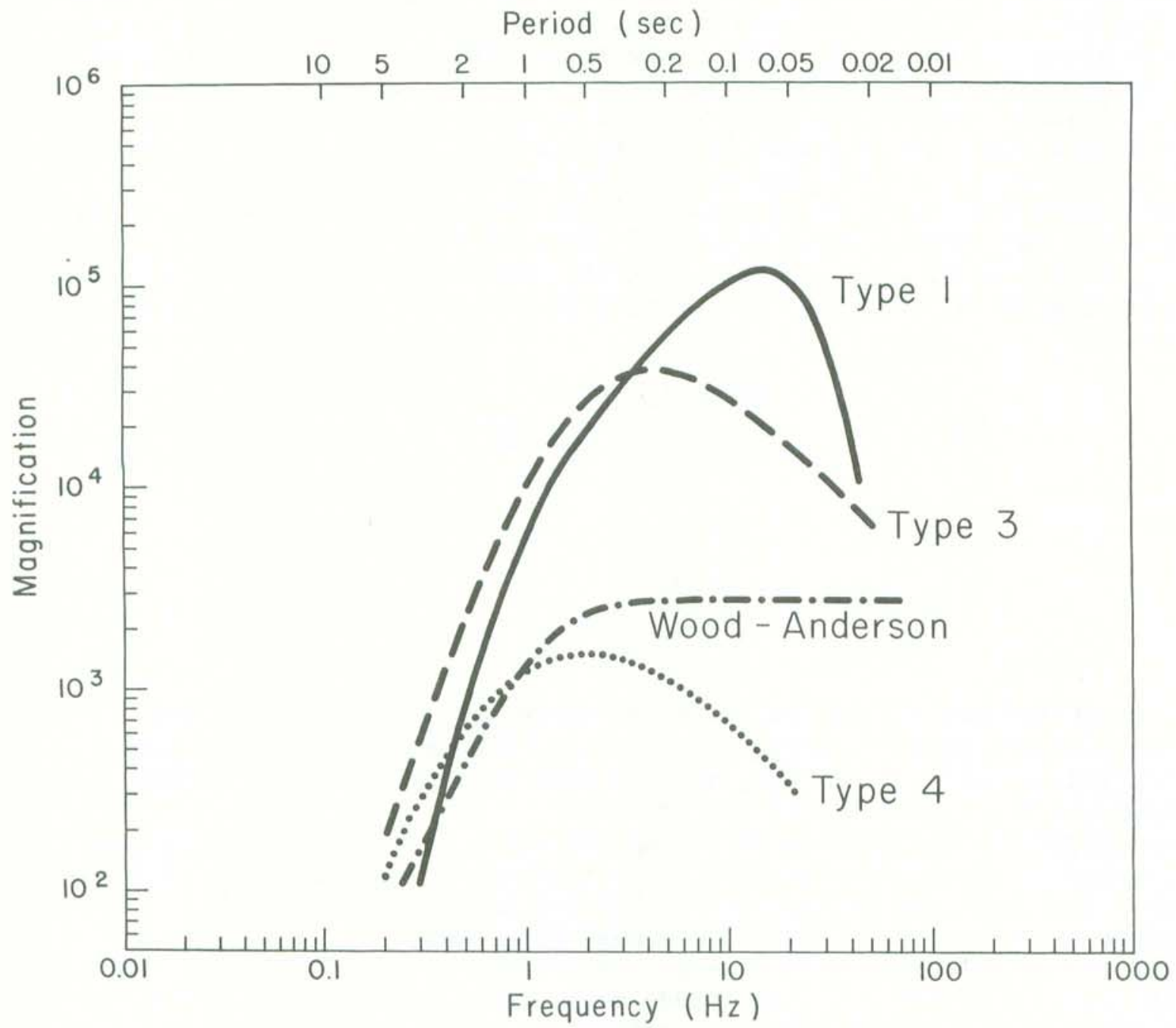

Figure 3. System response curves for the Wood-Anderson torsion seismograph and for the 3 different types of seismometers in use by the Hawaiian Volcano Observatory. Types 3 and 4 are electro-mechanical seismographs recorded optically on photographic paper. Type 1 is the standard NCER seismometer recorded on Develocorder film. The curve for Type 1 includes response of the geophone, all electronics including telemetry, Develocorder galvanometer, and projection of film by a $20 \mathrm{X}$ viewer. The curves plot the unit response which should be multiplied by the factors CAL listed in Table 1 to get the response for an individual station. 


\section{SEISMIC DATA PROCESSING}

Earthquakes are located by the combined efforts of HVO and NCER in Menlo Park. Develocorder films are read at HVO, and lists of $\mathrm{P}$ and $\mathrm{S}$ arrival times, event amplitude and duration, clock correction, etc. are sent to Menlo Park. Data are then keypunched, computer locations are made using the program HYPOELLIPSE (Lahr, et al., in preparation), and problem events are reread at HVO and rerun. Card and magnetic tape copies of all arrival time (phase) and output summary data (one card per event), are kept in Menlo Park. All computer output (including first motion plots) are on microfiche, and copies are available for inspection at HVO and in Menlo Park.

The crustal model used consists of flat, homogeneous layers and contains a embedded low velocity zone. It is a modified version of Crosson's (1976) model and is:

$\begin{array}{ccc}\text { LAYER } & \begin{array}{c}\text { VELOCITY } \\ (\mathrm{km} / \mathrm{sec})\end{array} & \text { DEPTH TO TOP }(\mathrm{km}) \\ 2 & 3.00 & 0.0 \\ 2 & 3.25 & 0.8 \\ 3 & 6.00 & 2.0 \\ 4 & 6.40 & 5.5 \\ 5 & 5.30 & 9.5 \\ 6 & 8.30 & 13.0\end{array}$

An empirical set of station delays or corrections were used in the locations, and are given in Table 1. They have been adjusted so the mean delay of Kilauea stations is zero, and are most appropriate for locating earthquakes on the south side of the island.

Magnitudes for most events were computed using both recorded amplitudes on calibrated stations and signal or coda duration on short-period vertical stations. Amplitude magnitudes used by $\mathrm{HVO}$ are based on readings from Wood-Anderson seismographs. Amplitudes read from other instruments are corrected to an equivalent Wood-Anderson amplitude using the curves of Figure 3 and CAL factors of Table 1. Amplitude magnitudes larger than 2.5 are generally based on the Wood-Anderson instruments in Hilo or Type 4 seismographs at Uwekahuna. Smaller events may occasionally include amplitude readings from stations AHU, KAA, OTL, or PPL.

Duration magnitudes are determined from the length of signal in seconds read from the Develocorder viewer. This time, also called the "F-P time" is measured from the first $P$ arrival to the point where the earthquake signal has decayed to about twice the noise level, or to about $1 \mathrm{~cm}$ peak-to-peak on the Develocorder viewer. A plot of log (F-P time) versus local (amplitude) magnitude appears in Figure 4. The bilinear relation shown in the figure is an appropriate fit to the data sample and is used to compute all duration magnitudes. Duration times are only read from Type 1 seismographs. Because duration magnitudes are relatively insensitive to station response and can be determined using the high-gain short-period stations, it is felt that duration magnitudes are more accurate and complete at the lower magnitudes (below 2). 


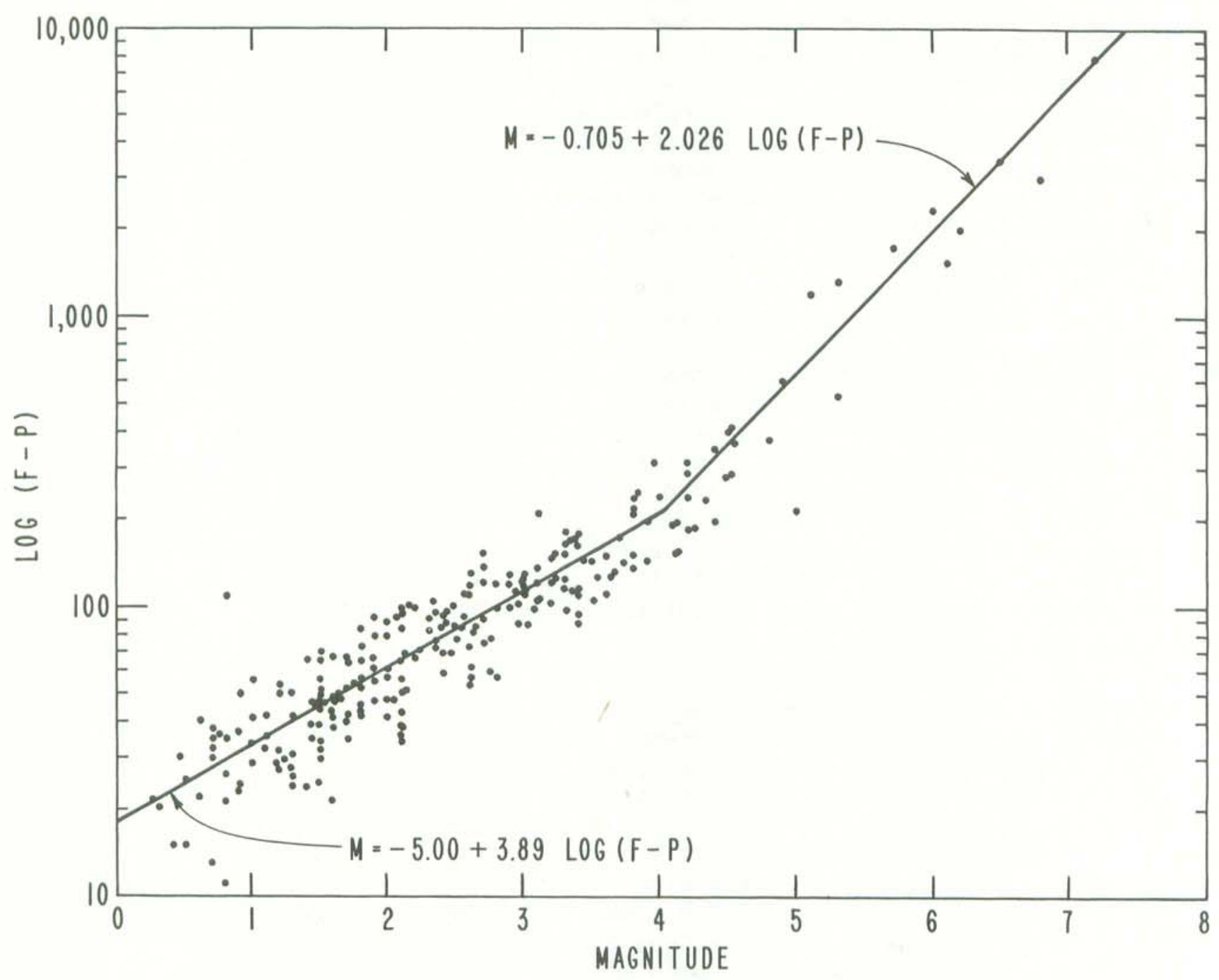

Figure 4. Relationship between signal duration (F-P time) and local magnitude for a large number of earthquakes which occurred during 1975 and 1976. Local magnitude is determined from amplitudes read on Wood-Anderson and other calibrated seismographs. The dual linear relationship between magnitude and $\log (\mathrm{F}-\mathrm{P})$ appears to hold over a magnitude range of 7 units. 


\section{SEISMIC SUMMARY}

The emphasis in both station coverage and detailed data analysis is on the highly active south flank of the island of Hawaii. Hundreds of earthquakes too small to locate are counted daily, and the set of located earthquakes in the Kilauea region is nearly complete above magnitude about 2.0 to 2.5. Many smaller events are located also. Substantial effort is made to locate earthquakes elsewhere on the island and within about $150 \mathrm{~km}$ of the island. Such coverage cannot be as complete as on the south flank, but nearly all events above magnitude 3.0 to 3.5 are located.

Data presented in the seismic summary is in four parts. Table 3 gives duration of harmonic tremor and numbers of earthquakes (most too small to locate) from several source regions around Kilauea. The source region is determined visually from signal character and pattern of arrival times at key stations. Maps showing computer located epicenters of all depths are given in Figures 5, 6, 7 and 8. The epicenter maps are on two scales, and show both all located earthquakes and large events only.

The list of computer locations constitutes the bulk of this summary, and is given in Table 5. Each earthquake in the list is assigned a three-letter code based on its location and in some cases on its depth. Figure 9 is a map of the regions used to assign the location codes. The latitude and longitude limits of rectangular regions are listed in Table 4 . When the listed coordinates imply an overlap, precedence is given according to Figure 9. Table 6 relists the events in Table 5 for which either duration or amplitude magnitude is 3.5 or larger. It is felt that this list is a more objective measure of large earthquakes than a list of felt earthquakes.

Table 3. Number of earthquakes and minutes of tremor recorded on seismographs around Kilauea. Tremor is separated into three categories: Deep, Intermediate, and Shallow, on the basis of relative amplitude on seismographs in the summit region. Unless otherwise stated, tremor is presumed to be associated with movement of magma within the central complex of Kilauea Volcano. Earthquake categories are: Kilauea Summit $30 \mathrm{~km}$, earthquakes from about $30 \mathrm{~km}$ beneath the summit region; Kilauea Summit long-period, earthquakes characterized by low-frequency waves from intermediate depths roughly 5-10 km beneath the summit region: Kilauea Summit Shallow, earthquakes from within a few $\mathrm{km}$ beneath the caldera region; SW Rift and Kaoiki, earthquakes along the southwest rift zone of Kilauea and the adjacent portions of the Kaoiki fault system; Upper East Rift, earthquakes from the upper east rift zone of Kilauea; Koae, earthquake from along the northeast-trending Koae fault system south of the caldera; Lower East Rift, earthquakes from the lower east rift zone of Kilauea; South Flank, faults on the south flank of Kilauea; Mauna Loa L-P, earthquakes characterized by low-frequency waves from Mauna Loa volcano; Mauna Loa S-P earthquakes from within a few kilometers beneath the summit of Mauna Loa; Offshore PPL, earthquakes from mostly offshore areas south of Puu Pili station. 


\begin{tabular}{|c|c|c|c|c|c|c|c|c|c|c|c|c|c|c|}
\hline \multirow{3}{*}{$\begin{array}{l}\text { Date } \\
(1976)\end{array}$} & \multirow{2}{*}{\multicolumn{3}{|c|}{$\begin{array}{c}\text { Tremor } \\
(\mathrm{m}=\text { minutes } \\
\mathrm{h}=\text { hours })\end{array}$}} & \multicolumn{11}{|c|}{ Earthquakes } \\
\hline & & & & \multicolumn{3}{|c|}{ Kilauea Summit } & \multicolumn{4}{|c|}{ Kilauea Flank } & \multicolumn{2}{|c|}{ Mauna Loa } & \multirow[b]{2}{*}{$\begin{array}{l}\text { Off- } \\
\text { shore } \\
\text { PPL }\end{array}$} & \multirow[b]{2}{*}{$\begin{array}{c}\text { Remarks and Events } \\
\text { of Interest }\end{array}$} \\
\hline & Deep & $\begin{array}{l}\text { Inter- } \\
\text { mediate }\end{array}$ & Shallow & $30 \mathrm{KM}$ & L-P & $S-P$ & $\begin{array}{l}\text { SW Rift } \\
\text { and } \\
\text { Kaoiki }\end{array}$ & $\mid \begin{array}{l}\text { Upper } \\
\text { East } \\
\text { Rift }\end{array}$ & Koae & $\begin{array}{l}\text { Lower } \\
\text { East } \\
\text { Rift }\end{array}$ & $L-P$ & $S-P$ & & \\
\hline $\begin{aligned} & \text { Feb } 1 \\
& 2 \\
& 3 \\
& 3 \\
& 4 \\
& 5 \\
& 6 \\
& 6 \\
& 7 \\
& 8 \\
& 9 \\
& 10 \\
& 11 \\
& 12 \\
& 13 \\
& 14 \\
& 15 \\
& 16 \\
& 17 \\
& 18 \\
& 19 \\
& 20 \\
& \\
& 21 \\
& 22 \\
& 23 \\
& 24 \\
& 25 \\
& 26 \\
& 27 \\
& 28 \\
&\end{aligned}$ & $\begin{array}{r}4 \mathrm{~m} \\
2 \mathrm{~m} \\
36 \mathrm{~m} \\
5 \mathrm{~m} \\
9 \mathrm{~m}\end{array}$ & $\begin{array}{l}5 \mathrm{~m} \\
3 \mathrm{~m} 1 /\end{array}$ & $\begin{array}{l}2 \mathrm{ml} / \\
10 \mathrm{~m} \\
10 \mathrm{~m}\end{array}$ & $\begin{array}{l}1 \\
1 \\
3 \\
4 \\
\\
2 \\
1\end{array}$ & $\begin{array}{r}11 \\
23 \\
23 \\
13 \\
3 \\
15 \\
11 \\
8 \\
2 \\
24 \\
6 \\
7 \\
1 \\
\\
\\
7 \\
7 \\
9 \\
10 \\
22 \\
8 \\
23 \\
1 \\
6 \\
26 \\
14 \\
8 \\
12 \\
25\end{array}$ & $\begin{array}{r}123 \\
146 \\
141 \\
192 \\
72 \\
155 \\
87 \\
100 \\
86 \\
326 \\
208 \\
146 \\
67 \\
104 \\
71 \\
122 \\
150 \\
183 \\
202 \\
141 \\
\\
98 \\
118 \\
64 \\
120 \\
511 \\
263 \\
143\end{array}$ & $\begin{array}{r}326 \\
246 \\
261 \\
167 \\
242 \\
237 \\
216 \\
228 \\
213 \\
183 \\
231 \\
175 \\
41 \\
174 \\
31 \\
123 \\
150 \\
165 \\
219 \\
145 \\
\\
202 \\
221 \\
126 \\
121 \\
119 \\
64 \\
52 \\
65 \\
177 \\
\end{array}$ & $\begin{array}{l}934 \\
995 \\
985 \\
790 \\
582 \\
691 \\
752 \\
776 \\
807 \\
903 \\
874 \\
600 \\
222 \\
530 \\
253 \\
612 \\
646 \\
732 \\
779 \\
650 \\
\\
551 \\
593 \\
717 \\
655 \\
750 \\
627 \\
858 \\
835 \\
857\end{array}$ & $\begin{array}{r}134 \\
143 \\
151 \\
69 \\
87 \\
38 \\
86 \\
90 \\
92 \\
161 \\
150 \\
97 \\
25 \\
54 \\
43 \\
68 \\
68 \\
90 \\
74 \\
41 \\
\\
43 \\
41 \\
56 \\
65 \\
27 \\
15 \\
20 \\
25 \\
74\end{array}$ & $\begin{array}{r}110 \\
215 \\
241 \\
65 \\
107 \\
91 \\
126 \\
74 \\
71 \\
70 \\
72 \\
67 \\
70 \\
93 \\
59 \\
50 \\
62 \\
60 \\
48 \\
79 \\
\\
73 \\
59 \\
34 \\
31 \\
99 \\
51 \\
99 \\
98 \\
97\end{array}$ & $\begin{array}{l}3 \\
1 \\
1 \\
2 \\
3 \\
4 \\
4 \\
1 \\
\\
2 \\
2 \\
8 \\
3 \\
3 \\
2 \\
4 \\
5 \\
1 \\
2 \\
2 \\
4 \\
6\end{array}$ & $\begin{array}{r}1 \\
3 \\
7 \\
4 \\
5 \\
11 \\
7 \\
1 \\
4 \\
4 \\
10 \\
4 \\
8 \\
5 \\
6 \\
\\
8 \\
12 \\
2 \\
3 \\
1\end{array}$ & $\begin{array}{l}1 \\
9 \\
1 \\
3\end{array}$ & $\begin{array}{l}\text { Mauna Kea-1, Kona-2 } \\
\text { LPD-1 } \\
\text { Heavy rains, LPD-1 } \\
\text { Electrical storm } \\
15 \text { km-1, LPD-1 } \\
\text { Kona-2 } \\
\text { LPD-1 } \\
\text { NER-2, Kona-1 } \\
\text { LPD-2, Kona-1 } \\
\text { High winds } \\
\text { Heavy rains } \\
\text { High winds } \\
\text { Heavy rains, Kona-1 } \\
\text { Kona-1 } \\
\text { Kohala-1, LPD-1, } \\
\text { Kona-3 } \\
\text { LPD-2 } \\
\text { Kona-1 } \\
\text { NER-2 } \\
\text { LPD-1, NER-5 } \\
\text { NER-4, Kona-1 } \\
\text { NER-3 } \\
\text { NER (2), Kona (2), } \\
\text { Electrical storm }\end{array}$ \\
\hline
\end{tabular}

1/MOK-Tremor beneath Mauna Loa Volcano 


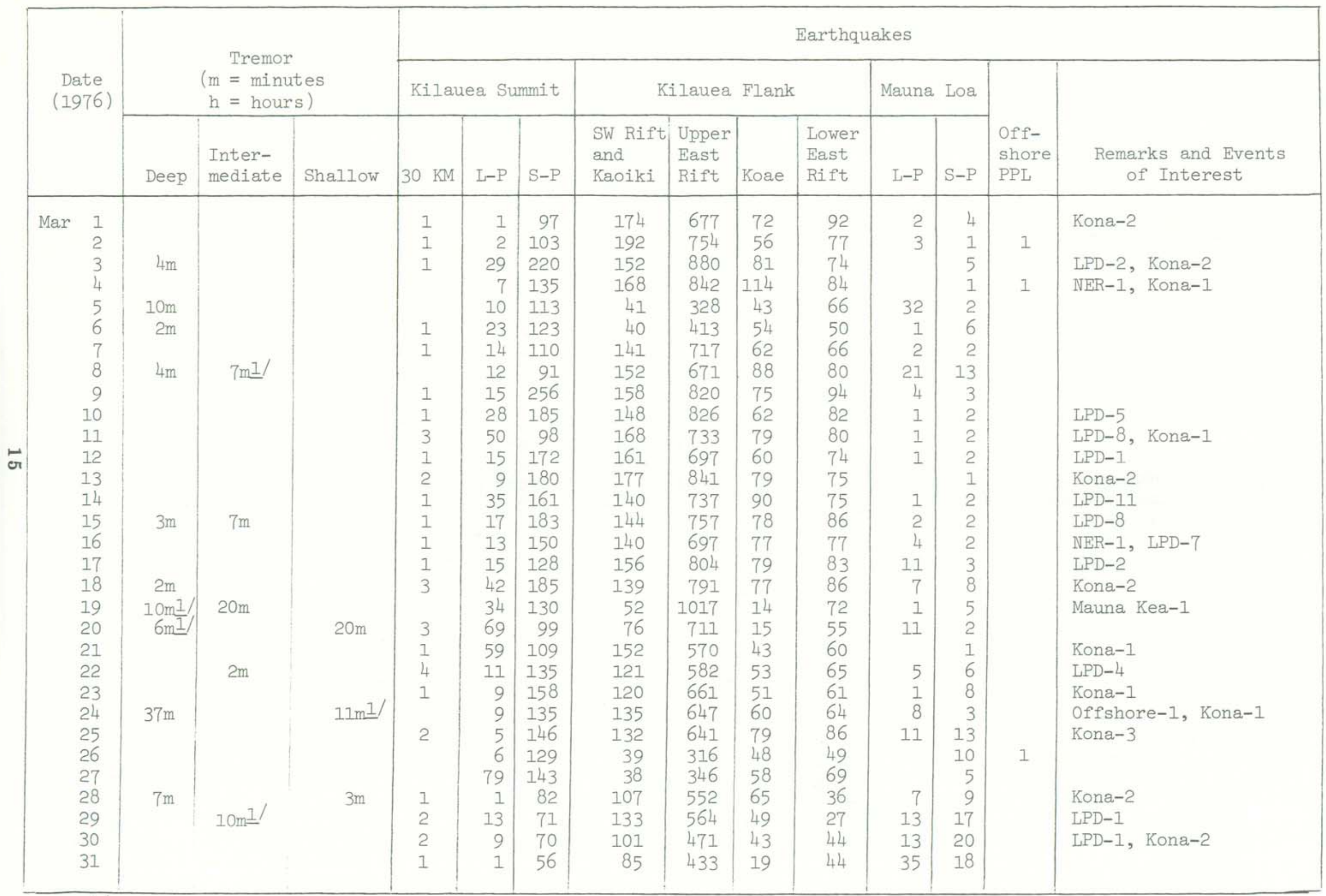

1/MOK-tremor beneath Mauna Loa Volcano 


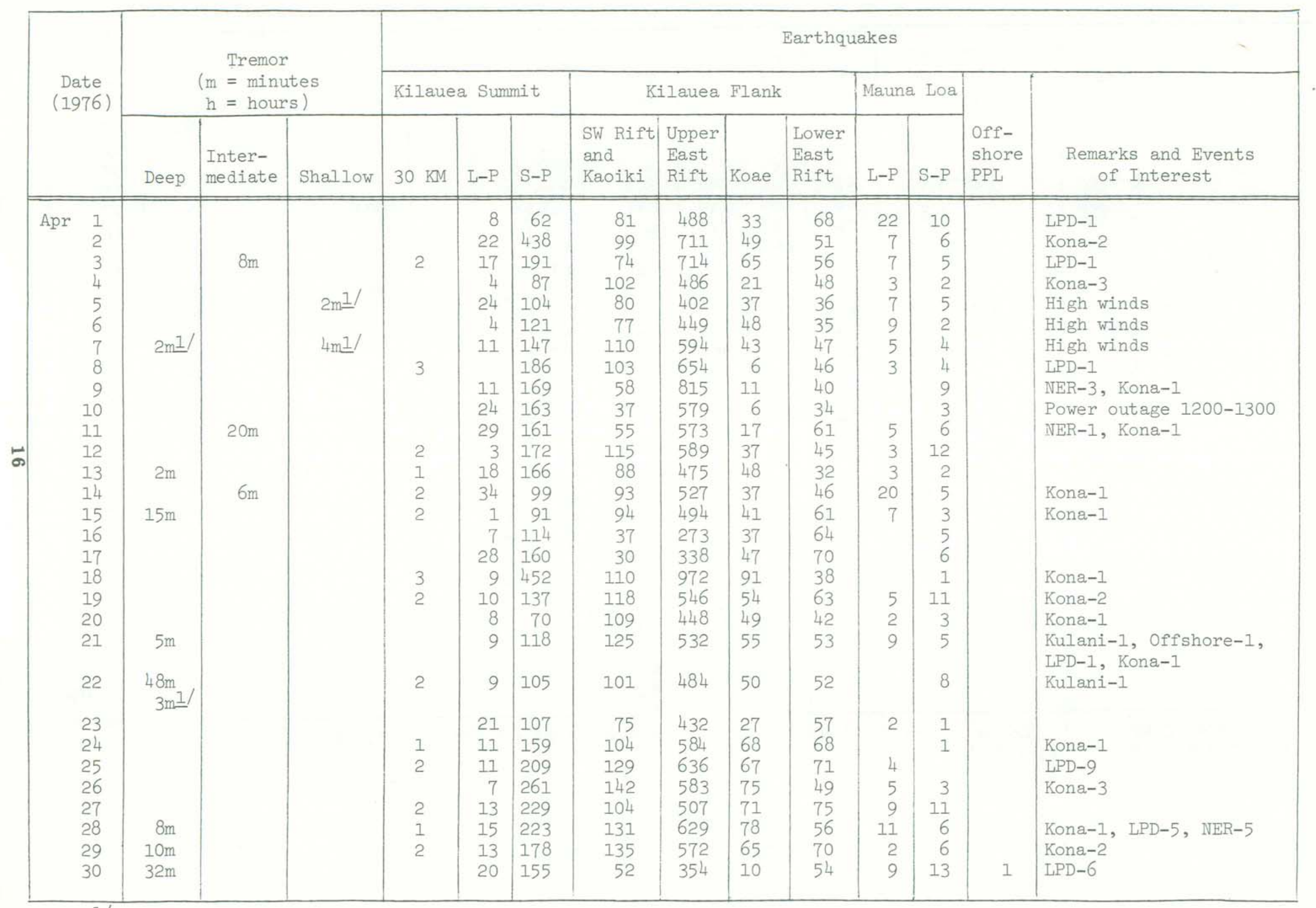




\begin{tabular}{|c|c|c|c|c|c|c|c|c|c|c|c|c|c|c|}
\hline \multirow{3}{*}{$\begin{array}{l}\text { Date } \\
(1976)\end{array}$} & \multirow{2}{*}{\multicolumn{3}{|c|}{$\begin{array}{c}\text { Tremor } \\
(\mathrm{m}=\text { minutes } \\
\mathrm{h}=\text { hours })\end{array}$}} & \multicolumn{11}{|c|}{ Earthquakes } \\
\hline & & & & \multicolumn{3}{|c|}{ Kilauea Summit } & \multicolumn{4}{|c|}{ Kilauea Flank } & \multicolumn{2}{|c|}{ Mauna Loa } & \multirow[b]{2}{*}{$\begin{array}{l}\text { Off- } \\
\text { shore } \\
\text { PPL }\end{array}$} & \multirow[b]{2}{*}{$\begin{array}{l}\text { Remarks and Events } \\
\text { of Interest }\end{array}$} \\
\hline & Deep & $\begin{array}{l}\text { Inter- } \\
\text { mediate }\end{array}$ & Shallow & $30 \mathrm{KM}$ & L-P & $\mathrm{S}-\mathrm{P}$ & $\begin{array}{l}\text { SW Rift. } \\
\text { and } \\
\text { Kaoiki }\end{array}$ & $\begin{array}{l}\text { Upper } \\
\text { East } \\
\text { Rift }\end{array}$ & Koae & $\begin{array}{l}\text { Lower } \\
\text { East } \\
\text { Rift }\end{array}$ & $\mathrm{I}-\mathrm{P}$ & $S-P$ & & \\
\hline $\begin{array}{r}\text { May } \\
1 \\
2 \\
3 \\
4 \\
5 \\
\\
6 \\
7 \\
8 \\
9 \\
\\
10 \\
11 \\
12 \\
13 \\
14 \\
15 \\
16 \\
17 \\
18 \\
19 \\
20 \\
21 \\
22 \\
23 \\
24 \\
25 \\
26 \\
27 \\
28 \\
29 \\
30 \\
31\end{array}$ & $\begin{array}{l}69 \mathrm{~m} \\
174 \mathrm{~m} \\
22 \mathrm{~m}\end{array}$ & $\begin{array}{l}10 \mathrm{~m} \\
2 \mathrm{~m} 1 /\end{array}$ & $10 \mathrm{~m} \perp /$ & $\begin{array}{l}3 \\
1 \\
1 \\
2\end{array}$ & $\begin{array}{r}23 \\
9 \\
12 \\
14 \\
5 \\
4 \\
1 \\
\\
2 \\
\\
8 \\
32 \\
37 \\
437 \\
50 \\
148 \\
47 \\
49 \\
8 \\
1 \\
3 \\
6 \\
1 \\
5 \\
2 \\
9 \\
2 \\
1 \\
2 \\
1 \\
13\end{array}$ & \begin{tabular}{|r}
177 \\
190 \\
152 \\
161 \\
172 \\
196 \\
160 \\
182 \\
117 \\
157 \\
182 \\
173 \\
136 \\
149 \\
226 \\
94 \\
199 \\
126 \\
222 \\
234 \\
204 \\
219 \\
244 \\
237 \\
274 \\
286 \\
257 \\
382 \\
326 \\
386 \\
290
\end{tabular} & $\begin{array}{r}132 \\
133 \\
106 \\
87 \\
87 \\
\\
85 \\
20 \\
29 \\
94 \\
\\
86 \\
86 \\
27 \\
23 \\
35 \\
46 \\
97 \\
106 \\
97 \\
88 \\
71 \\
86 \\
86 \\
61 \\
75 \\
82 \\
88 \\
85 \\
32 \\
32 \\
57 \\
113\end{array}$ & $\begin{array}{l}424 \\
\\
458 \\
543 \\
504 \\
633 \\
\\
503 \\
249 \\
258 \\
471 \\
\\
557 \\
474 \\
237 \\
233 \\
389 \\
429 \\
486 \\
462 \\
397 \\
588 \\
317 \\
373 \\
350 \\
405 \\
388 \\
446 \\
457 \\
396 \\
227 \\
385 \\
408 \\
525\end{array}$ & $\begin{array}{l}54 \\
77 \\
61 \\
42 \\
50 \\
\\
64 \\
51 \\
53 \\
36 \\
\\
64 \\
53 \\
10 \\
4 \\
7 \\
5 \\
44 \\
67 \\
70 \\
62 \\
32 \\
45 \\
34 \\
47 \\
38 \\
37 \\
56 \\
42 \\
50 \\
66 \\
77 \\
55\end{array}$ & $\begin{array}{r}65 \\
104 \\
83 \\
66 \\
72 \\
\\
86 \\
49 \\
79 \\
64 \\
\\
76 \\
81 \\
20 \\
52 \\
67 \\
37 \\
77 \\
97 \\
87 \\
87 \\
66 \\
59 \\
42 \\
50 \\
63 \\
75 \\
95 \\
61 \\
58 \\
36 \\
38 \\
22\end{array}$ & $\begin{array}{r}26 \\
4 \\
10 \\
20 \\
5 \\
19 \\
10 \\
18 \\
17 \\
8 \\
1 \\
6 \\
4 \\
32 \\
12 \\
11 \\
17 \\
11 \\
10 \\
12 \\
7 \\
15 \\
11 \\
9 \\
5 \\
1 \\
1\end{array}$ & $\begin{array}{r}9 \\
3 \\
9 \\
3 \\
6 \\
33 \\
43 \\
26 \\
10 \\
\\
8 \\
8 \\
16 \\
2 \\
2 \\
2 \\
14 \\
9 \\
3 \\
87 \\
6 \\
3 \\
4 \\
8 \\
4 \\
2 \\
2 \\
5 \\
3 \\
6 \\
1\end{array}$ & 1 & $\begin{array}{l}\text { Kona-1, Mauna Kea-1 } \\
\text { LPD-53, NER-1 } \\
\text { Kona-3, LPD-12, NER-1 } \\
\text { Kona-3, LPD-19, NER-5 } \\
\text { LPD-10, Kona-2 } \\
\text { Kulani-3, LPD-4, NER-6, } \\
\text { Kona-3 } \\
\text { NER-1, Kona-1 } \\
\text { Mauna Kea-1, NER-2, } \\
\text { Kona-3 } \\
\text { NER-2, Kona-1 } \\
\text { NER-4 } \\
\text { Kona-1, Mauna Kea-1 } \\
\\
\text { Kona-2 } \\
\text { LPD-5, Kona-1 } \\
\text { Mauna Kea-1, 15 km-1 } \\
\text { NER-1, Kona-1 } \\
\text { LPD-7, Kona-1 } \\
\text { LPD-4 } \\
\text { NER-1 } \\
\text { NER-1 } \\
\text { LPD-6 } \\
\text { Kohala-7 }\end{array}$ \\
\hline
\end{tabular}




\begin{tabular}{|c|c|c|c|c|c|c|c|c|c|c|c|c|c|c|}
\hline \multirow{3}{*}{$\begin{array}{l}\text { Date } \\
\text { (1976) }\end{array}$} & \multirow{2}{*}{\multicolumn{3}{|c|}{$\begin{array}{c}\text { Tremor } \\
(\mathrm{m}=\text { minutes } \\
\mathrm{h}=\text { hours })\end{array}$}} & \multicolumn{11}{|c|}{ Earthquakes } \\
\hline & & & & \multicolumn{3}{|c|}{ Kilauea Summit } & \multicolumn{4}{|c|}{ Kilauea Flank } & \multicolumn{2}{|c|}{ Mauna Loa } & \multirow[b]{2}{*}{$\begin{array}{l}\text { Off- } \\
\text { shore } \\
\text { PPL }\end{array}$} & \multirow[b]{2}{*}{$\begin{array}{c}\text { Remarks and Events } \\
\text { of Interest }\end{array}$} \\
\hline & Deep & $\begin{array}{l}\text { Inter- } \\
\text { mediate }\end{array}$ & Shallow & $30 \mathrm{KM}$ & $\mathrm{L}-\mathrm{P}$ & S-P & $\begin{array}{l}\text { SW Rift } \\
\text { and } \\
\text { Kaoiki }\end{array}$ & $\mid$\begin{tabular}{|l|} 
Upper \\
East \\
Rift
\end{tabular} & Koae & $\begin{array}{l}\text { Lower } \\
\text { East } \\
\text { Rift }\end{array}$ & $\mathrm{L}-\mathrm{P}$ & $S-P$ & & \\
\hline $\begin{array}{r}1 \\
2 \\
3 \\
4 \\
4 \\
5 \\
6 \\
7 \\
8 \\
9 \\
10 \\
11 \\
12 \\
13 \\
14 \\
15 \\
16 \\
17 \\
18 \\
19 \\
20 \\
21 \\
22 \\
23 \\
24 \\
25 \\
26 \\
27 \\
28 \\
29 \\
30\end{array}$ & $\begin{array}{r}5 \mathrm{~m} \\
2 \mathrm{~m} \\
22 \mathrm{~m} \\
16 \mathrm{~m} \\
10 \mathrm{~m} \\
2 \mathrm{~m} \\
11 \mathrm{~m} \\
5 \mathrm{~m} \\
3 \mathrm{~m} \\
\\
5 \mathrm{~m} \\
6 \mathrm{~m}\end{array}$ & $\begin{array}{c}7 \mathrm{~m} 1 / \\
4 \mathrm{~m} 1 / \\
\\
\\
15 \mathrm{~m} \\
8 \mathrm{~m}\end{array}$ & $5 \mathrm{~m} 1 /$ & $\begin{array}{l}4 \\
7 \\
1 \\
3 \\
1 \\
1 \\
1 \\
1 \\
1\end{array}$ & $\begin{array}{r}7 \\
56 \\
78 \\
91 \\
302 \\
107 \\
10 \\
10 \\
14 \\
79 \\
98 \\
44 \\
4 \\
6 \\
5 \\
3 \\
3 \\
6 \\
5 \\
4 \\
6 \\
27 \\
14 \\
20 \\
15\end{array}$ & $\begin{array}{l}325 \\
308 \\
271 \\
332 \\
299 \\
341 \\
276 \\
312 \\
326 \\
335 \\
294 \\
347 \\
344 \\
241 \\
210 \\
144 \\
207 \\
205 \\
286 \\
352 \\
142 \\
133 \\
126 \\
163 \\
218 \\
298 \\
277 \\
231 \\
263 \\
245\end{array}$ & $\begin{array}{r}86 \\
84 \\
109 \\
105 \\
91 \\
127 \\
74 \\
85 \\
52 \\
56 \\
28 \\
23 \\
86 \\
86 \\
71 \\
63 \\
52 \\
53 \\
54 \\
67 \\
39 \\
73 \\
87 \\
71 \\
20 \\
30 \\
97 \\
72 \\
78 \\
73\end{array}$ & $\begin{array}{l}509 \\
590 \\
552 \\
686 \\
498 \\
427 \\
393 \\
448 \\
579 \\
544 \\
429 \\
445 \\
519 \\
373 \\
460 \\
299 \\
319 \\
387 \\
479 \\
423 \\
3134 \\
828 \\
660 \\
382 \\
253 \\
257 \\
531 \\
440 \\
432 \\
396\end{array}$ & $\begin{array}{l}49 \\
68 \\
62 \\
76 \\
47 \\
52 \\
51 \\
42 \\
30 \\
78 \\
18 \\
14 \\
55 \\
44 \\
47 \\
34 \\
36 \\
42 \\
73 \\
53 \\
22 \\
59 \\
23 \\
11 \\
54 \\
64 \\
34 \\
20 \\
25 \\
19\end{array}$ & $\begin{array}{l}31 \\
60 \\
71 \\
52 \\
34 \\
58 \\
47 \\
73 \\
78 \\
61 \\
39 \\
3 \\
56 \\
38 \\
33 \\
36 \\
43 \\
39 \\
35 \\
34 \\
29 \\
56 \\
51 \\
53 \\
31 \\
31 \\
52 \\
32 \\
34 \\
44\end{array}$ & $\begin{array}{r}7 \\
7 \\
1 \\
3 \\
2 \\
5 \\
3 \\
2 \\
2 \\
7 \\
5 \\
1 \\
3 \\
2 \\
6 \\
6 \\
22 \\
12 \\
8 \\
10 \\
29 \\
14 \\
6 \\
2 \\
7 \\
17 \\
2 \\
31\end{array}$ & $\begin{array}{r}1 \\
3 \\
1 \\
1 \\
2 \\
1 \\
1 \\
1 \\
1 \\
1 \\
\\
3 \\
2 \\
2 \\
2 \\
2 \\
5 \\
6 \\
17 \\
6 \\
8 \\
8 \\
10 \\
8 \\
2 \\
7 \\
9 \\
2 \\
5 \\
3\end{array}$ & & $\begin{array}{l}\text { LPD-4, Kona-1 } \\
\text { NER-1 } \\
\text { Kona-1 } \\
\text { Kona-2 } \\
\text { Kohala-1, Kona-1 } \\
\text { Kohala-5 } \\
\text { Kona-1 } \\
\text { Mauna Kea-1 } \\
\text { Offshore-1, NER-8 } \\
\text { NER-4 } \\
\text { NER-2, Kona-1 } \\
\text { Kona-1 } \\
\text { NER-1, Kohala-1 } \\
\text { Kona-1 } \\
\text { Kohala-1 } \\
\text { NER-1 } \\
\text { Mauna Kea-1 } \\
\text { East Rift swarm } \\
\text { NER-5, Kona-2 } \\
\text { NER-2 } \\
\text { NER-1, Kona-1 } \\
\text { Kohala-1, Kona-1 } \\
\text { Kona-1 }\end{array}$ \\
\hline
\end{tabular}

1/MOK-tremor beneath Mauna Loa Volcano 


\begin{tabular}{|c|c|c|c|c|c|c|c|c|c|c|c|c|c|c|}
\hline \multirow{3}{*}{$\begin{array}{l}\text { Date } \\
(1976)\end{array}$} & \multirow{2}{*}{\multicolumn{3}{|c|}{$\begin{array}{c}\text { Tremor } \\
(\mathrm{m}=\text { minutes } \\
\mathrm{h}=\text { hours })\end{array}$}} & \multicolumn{11}{|c|}{ Earthquakes } \\
\hline & & & & \multicolumn{3}{|c|}{ Kilauea Summit } & \multicolumn{4}{|c|}{ Kilauea Flank } & \multicolumn{2}{|c|}{ Mauna Loa } & \multirow[b]{2}{*}{$\begin{array}{l}\text { Dff- } \\
\text { shore } \\
\text { PPL }\end{array}$} & \multirow[b]{2}{*}{$\begin{array}{l}\text { Remarks and Events } \\
\text { of Interest }\end{array}$} \\
\hline & Deep & $\begin{array}{l}\text { Inter- } \\
\text { mediate }\end{array}$ & Shallow & $30 \mathrm{KM}$ & $\mathrm{L}-\mathrm{P}$ & $\mathrm{S}-\mathrm{P}$ & $\begin{array}{l}\text { SW Rift } \\
\text { and } \\
\text { Kaoiki }\end{array}$ & $\begin{array}{l}\text { Upper } \\
\text { East } \\
\text { Rift }\end{array}$ & Koae & $\begin{array}{l}\text { Lower } \\
\text { East } \\
\text { Rift }\end{array}$ & $L-P$ & $S-P$ & & \\
\hline $\begin{array}{r}1 \\
2 \\
3 \\
4 \\
5 \\
6 \\
7 \\
8 \\
\\
9 \\
10 \\
11 \\
12 \\
13 \\
\\
14 \\
15 \\
16 \\
17 \\
18 \\
19 \\
20 \\
21 \\
22 \\
23 \\
24 \\
25 \\
26 \\
27 \\
28 \\
29 \\
30 \\
31\end{array}$ & $\begin{array}{c}6 \mathrm{~m} \\
24 \mathrm{~m} \\
15 \mathrm{~m} 1 / \\
8 \mathrm{~m} I /\end{array}$ & $\begin{array}{l}7 \mathrm{~m} \\
6 \mathrm{~m}\end{array}$ & $3 \mathrm{~m}^{1 /}$ & $\begin{array}{l}1 \\
2 \\
4 \\
2 \\
2 \\
2 \\
2 \\
3\end{array}$ & $\begin{array}{r}57 \\
17 \\
8 \\
16 \\
7 \\
9 \\
34 \\
28 \\
7 \\
5 \\
2 \\
27 \\
12 \\
9 \\
2 \\
3 \\
9 \\
20 \\
13 \\
16 \\
14\end{array}$ & $\begin{array}{r}228 \\
268 \\
282 \\
88 \\
349 \\
277 \\
117 \\
125 \\
\\
96 \\
150 \\
140 \\
119 \\
126 \\
\\
320 \\
105 \\
106 \\
132 \\
138 \\
167 \\
164 \\
114 \\
160 \\
329 \\
319 \\
193 \\
341 \\
221 \\
254 \\
245 \\
296 \\
302\end{array}$ & $\begin{array}{r}69 \\
72 \\
75 \\
6 \\
49 \\
59 \\
38 \\
46 \\
\\
22 \\
25 \\
47 \\
40 \\
42 \\
\\
62 \\
90 \\
55 \\
51 \\
46 \\
57 \\
62 \\
41 \\
57 \\
19 \\
49 \\
45 \\
63 \\
59 \\
64 \\
64 \\
70 \\
51\end{array}$ & $\begin{array}{r}380 \\
364 \\
401 \\
105 \\
395 \\
451 \\
188 \\
352 \\
\\
161 \\
315 \\
613 \\
636 \\
767 \\
\\
1677 \\
591 \\
534 \\
548 \\
435 \\
497 \\
376 \\
470 \\
478 \\
525 \\
732 \\
439 \\
422 \\
587 \\
560 \\
435 \\
568 \\
635\end{array}$ & $\begin{array}{r}21 \\
44 \\
33 \\
2 \\
31 \\
21 \\
17 \\
19 \\
\\
46 \\
39 \\
16 \\
28 \\
46 \\
\\
26 \\
41 \\
39 \\
41 \\
40 \\
48 \\
34 \\
30 \\
48 \\
19 \\
9 \\
33 \\
38 \\
23 \\
34 \\
53 \\
39 \\
51\end{array}$ & $\begin{array}{l}59 \\
45 \\
33 \\
10 \\
39 \\
42 \\
21 \\
26 \\
\\
32 \\
38 \\
36 \\
32 \\
32 \\
41 \\
\\
42 \\
44 \\
49 \\
47 \\
46 \\
29 \\
17 \\
31 \\
26 \\
21 \\
21 \\
29 \\
45 \\
28 \\
34 \\
34 \\
29 \\
30 \\
39 \\
29\end{array}$ & $\begin{array}{r}1 \\
4 \\
3 \\
3 \\
4 \\
2 \\
2 \\
1 \\
2 \\
2 \\
4 \\
2 \\
1 \\
9 \\
28 \\
6 \\
7\end{array}$ & $\begin{array}{l}5 \\
2 \\
1 \\
2 \\
2 \\
\\
2 \\
\\
37 \\
2 \\
9 \\
2 \\
2 \\
4 \\
2 \\
5 \\
4 \\
3 \\
1 \\
1 \\
1 \\
6 \\
3 \\
3 \\
3 \\
3 \\
1 \\
3 \\
3\end{array}$ & $\begin{array}{l}2 \\
1\end{array}$ & $\begin{array}{l}\text { LPD-2 } \\
\text { High winds } \\
\text { Kona-1 } \\
\text { Kona-1 } \\
\text { Power outage 2000-0700 } \\
\text { Power outage 0100-0730, } \\
\text { Mauna Kea-1 } \\
\text { Kona-1 } \\
\text { KOH-1, LPD-3 } \\
\text { Kona-1, KOH-1, LPD-1, } \\
\text { NER-3 } \\
\text { NER-1, Kona-2 } \\
\text { Kona-1, LPD-1, NER-1 } \\
\text { Kona-4, LPD-1, NER-1 } \\
\text { Kona-3, LPD-1, NER-5 } \\
\text { LPD-1, NER-4 } \\
\text { NER-4, LPD-1, Kona-1 } \\
\text { LPD-2 } \\
\text { LPD-1, NER-1 } \\
\text { LPD-1, Kona-1 } \\
\text { LPD-1 } \\
\text { Kona-1 } \\
\text { IPD-4 } \\
\text { LPD-2 } \\
\text { LPD-1, Kona-1 } \\
\text { LPD-2, Kona-1, NER-4 } \\
\text { LPD-1 }\end{array}$ \\
\hline
\end{tabular}




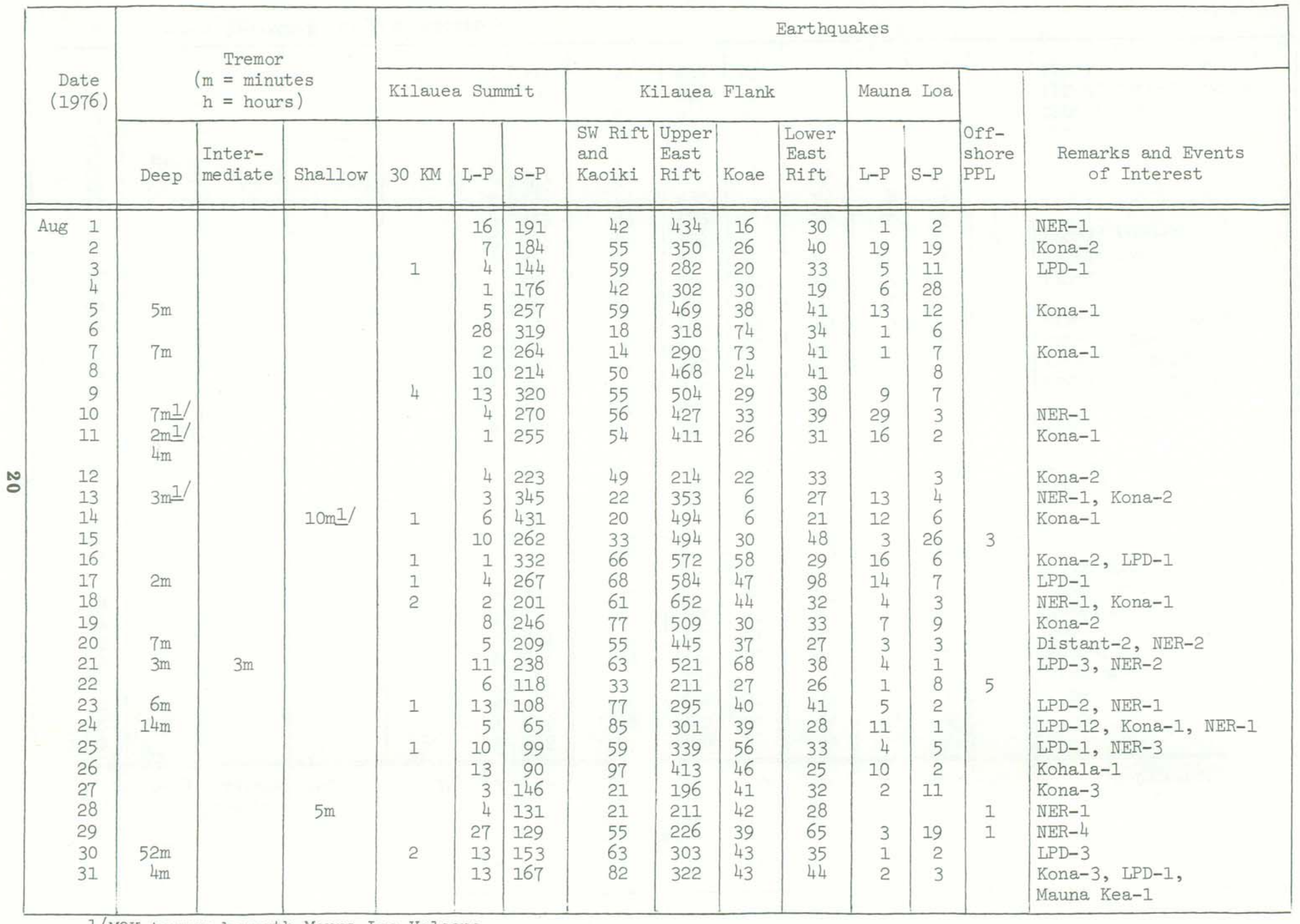




\begin{tabular}{|c|c|c|c|c|c|c|c|c|c|c|c|c|c|c|}
\hline \multirow{3}{*}{$\begin{array}{c}\text { Date } \\
(1976)\end{array}$} & \multirow{2}{*}{\multicolumn{3}{|c|}{$\begin{array}{c}\text { Tremor } \\
(\mathrm{m}=\text { minutes } \\
\mathrm{h}=\text { hours })\end{array}$}} & \multicolumn{11}{|c|}{ Earthquake } \\
\hline & & & & \multicolumn{3}{|c|}{ Kilauea Summit } & \multicolumn{4}{|c|}{ Kilauea Flank } & \multicolumn{2}{|c|}{ Mauna Loa } & \multirow[b]{2}{*}{$\begin{array}{l}\text { Off- } \\
\text { shore } \\
\text { PPL }\end{array}$} & \multirow[b]{2}{*}{$\begin{array}{l}\text { Remarks and Events } \\
\text { of Interest }\end{array}$} \\
\hline & Deep & $\begin{array}{l}\text { Inter- } \\
\text { mediate }\end{array}$ & Shallow & $30 \mathrm{KM}$ & $L-P$ & S-P & $\begin{array}{l}\text { SW Rift } \\
\text { and } \\
\text { Kaoiki }\end{array}$ & $\begin{array}{l}\text { Upper } \\
\text { East } \\
\text { Rift }\end{array}$ & Koae & $\begin{array}{l}\text { Lower } \\
\text { East } \\
\text { Rift }\end{array}$ & $\mathrm{L}-\mathrm{P}$ & S-P & & \\
\hline 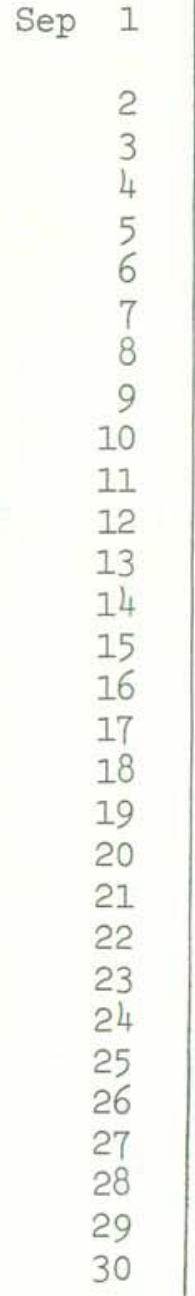 & $\begin{array}{l}4 \mathrm{~m}= \\
10 \mathrm{~m}\end{array}$ & $\begin{array}{c}9 \mathrm{~m} \\
3 \mathrm{~m} \\
20 \mathrm{~m} \\
4 \mathrm{~m} \\
11 \mathrm{~m} \\
5 \mathrm{~m} \\
3 \mathrm{~m}\end{array}$ & $\begin{array}{l}10 \mathrm{ml} / \\
15 \mathrm{~m}= \\
2 \mathrm{~m}\end{array}$ & $\begin{array}{l}2 \\
2 \\
2 \\
3 \\
\\
2 \\
1 \\
\\
2 \\
2 \\
1 \\
3 \\
1 \\
2\end{array}$ & $\begin{array}{r}11 \\
25 \\
35 \\
28 \\
25 \\
9 \\
20 \\
10 \\
7 \\
11 \\
11 \\
21 \\
7 \\
5 \\
3 \\
17 \\
7 \\
55 \\
16 \\
15 \\
3 \\
11 \\
27 \\
22 \\
127 \\
53 \\
32 \\
25 \\
23 \\
53\end{array}$ & $\begin{array}{r}117 \\
106 \\
125 \\
77 \\
83 \\
102 \\
83 \\
52 \\
72 \\
271 \\
363 \\
211 \\
171 \\
99 \\
75 \\
78 \\
82 \\
209 \\
99 \\
65 \\
52 \\
79 \\
87 \\
70 \\
81 \\
69 \\
70 \\
75 \\
88 \\
84\end{array}$ & $\begin{array}{l}76 \\
54 \\
43 \\
65 \\
69 \\
63 \\
79 \\
71 \\
56 \\
28 \\
12 \\
76 \\
54 \\
60 \\
39 \\
80 \\
74 \\
75 \\
72 \\
76 \\
75 \\
67 \\
76 \\
24 \\
28 \\
68 \\
75 \\
87 \\
81\end{array}$ & $\begin{array}{l}359 \\
375 \\
300 \\
172 \\
258 \\
365 \\
384 \\
261 \\
225 \\
286 \\
291 \\
334 \\
320 \\
197 \\
212 \\
299 \\
330 \\
339 \\
340 \\
287 \\
278 \\
352 \\
342 \\
155 \\
131 \\
348 \\
321 \\
394 \\
382 \\
421\end{array}$ & $\begin{array}{r}40 \\
59 \\
64 \\
44 \\
52 \\
43 \\
52 \\
15 \\
23 \\
3 \\
1 \\
35 \\
34 \\
5 \\
8 \\
26 \\
49 \\
40 \\
38 \\
26 \\
49 \\
38 \\
40 \\
42 \\
38 \\
29 \\
26 \\
47 \\
40\end{array}$ & $\begin{array}{r}27 \\
36 \\
84 \\
48 \\
45 \\
33 \\
31 \\
29 \\
33 \\
56 \\
171 \\
200 \\
116 \\
71 \\
62 \\
83 \\
80 \\
56 \\
82 \\
69 \\
36 \\
41 \\
47 \\
45 \\
35 \\
46 \\
44 \\
31 \\
42 \\
30\end{array}$ & $\begin{array}{r}2 \\
29 \\
40 \\
4 \\
4 \\
4 \\
4 \\
3 \\
10 \\
10 \\
5 \\
13 \\
17 \\
10 \\
6 \\
4 \\
12 \\
29 \\
31 \\
16 \\
15 \\
16 \\
2 \\
14 \\
5 \\
3 \\
1 \\
10\end{array}$ & $\begin{array}{r}6 \\
15 \\
10 \\
5 \\
2 \\
3 \\
\\
1 \\
10 \\
1 \\
\\
4 \\
2 \\
4 \\
3 \\
1 \\
2 \\
1 \\
1 \\
2 \\
9 \\
7 \\
3 \\
2 \\
2 \\
4\end{array}$ & $\begin{array}{l}1 \\
2 \\
1\end{array}$ & $\begin{array}{l}\text { NER-5, Mauna Kea-1, } \\
\text { Kona-1 } \\
\text { Mauna Kea-1, NER-4 } \\
\text { Kona-4, NER-1 } \\
\text { NER-2, Kona-1 } \\
\text { LPD-6, NER-1 } \\
\text { Mauna Kea-2 } \\
\text { Kona-1, LPD-1, NER-1 } \\
\text { LPD-1, Kona-2 } \\
\text { Kona-1 } \\
\text { LPD-2, NER-3 } \\
\text { Kohala-1 } \\
\text { LPD-2, Kona-3 } \\
\text { Mauna Kea-4 } \\
\text { Offshore-1, Kona-1 } \\
\text { LPD-2, Kohala-1 } \\
\text { NER-1 } \\
\text { LPD-4, Kona-3 } \\
\text { Kona-3 } \\
\text { NER-5, Kona-1 } \\
\text { Kona-1 } \\
\text { Kona-2, LPD-2, NER-1 } \\
\text { NER-1 } \\
\text { Kona-1 } \\
\text { Kona-1 }\end{array}$ \\
\hline
\end{tabular}

I/MOK-tremor beneath Mauna Loa Volcano 


\begin{tabular}{|c|c|c|c|c|c|c|c|c|c|c|c|c|c|c|c|}
\hline \multirow{3}{*}{\multicolumn{2}{|c|}{$\begin{array}{l}\text { Date } \\
(1976)\end{array}$}} & \multirow{2}{*}{\multicolumn{3}{|c|}{$\begin{array}{c}\text { Tremor } \\
(\mathrm{m}=\text { minutes } \\
\mathrm{h}=\text { hours })\end{array}$}} & \multicolumn{11}{|c|}{ Earthquakes } \\
\hline & & & & & \multicolumn{3}{|c|}{ Kilauea Summit } & \multicolumn{4}{|c|}{ Kilauea Flank } & \multicolumn{2}{|c|}{ Mauna Loa } & \multirow[b]{2}{*}{$\begin{array}{l}\text { Off- } \\
\text { shore } \\
\text { PPL }\end{array}$} & \multirow[b]{2}{*}{$\begin{array}{c}\text { Remarks and Events } \\
\text { of Interest }\end{array}$} \\
\hline & & Deep & $\begin{array}{l}\text { Inter- } \\
\text { mediate }\end{array}$ & Shallow & $30 \mathrm{KM}$ & $\mathrm{I}-\mathrm{P}$ & $S-P$ & $\begin{array}{l}\text { SW Rift } \\
\text { and } \\
\text { Kaoiki }\end{array}$ & $\begin{array}{l}\text { Upper } \\
\text { East } \\
\text { Rift }\end{array}$ & Koae & $\begin{array}{l}\text { Lower } \\
\text { East } \\
\text { Rift }\end{array}$ & L-P & $S-P$ & & \\
\hline \multicolumn{2}{|c|}{$\begin{array}{r}\text { Oct } \\
2 \\
2 \\
3 \\
3 \\
4 \\
5 \\
6 \\
7 \\
8 \\
9 \\
10 \\
11 \\
12 \\
13 \\
14 \\
15 \\
16 \\
17 \\
18 \\
19 \\
20 \\
21 \\
22 \\
23 \\
24 \\
25 \\
26 \\
27 \\
28 \\
29 \\
30 \\
31\end{array}$} & $\begin{array}{l}4 \mathrm{~m} \\
5 \mathrm{~m} 1 /\end{array}$ & 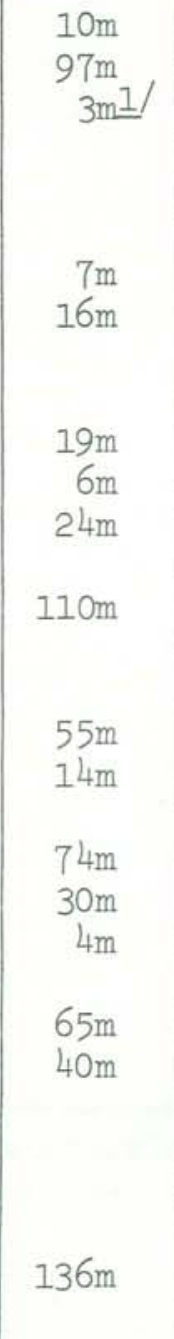 & & $\begin{array}{l}1 \\
4 \\
2 \\
\\
2 \\
3 \\
4 \\
3 \\
3 \\
1 \\
5\end{array}$ & $\begin{array}{r}150 \\
287 \\
58 \\
49 \\
26 \\
147 \\
128 \\
32 \\
30 \\
91 \\
46 \\
135 \\
36 \\
102 \\
9 \\
187 \\
121 \\
47 \\
23 \\
180 \\
111 \\
35 \\
27 \\
121 \\
101 \\
60 \\
6 \\
17 \\
7 \\
72 \\
20\end{array}$ & $\begin{array}{r}110 \\
\\
106 \\
114 \\
91 \\
108 \\
82 \\
95 \\
76 \\
106 \\
92 \\
175 \\
159 \\
175 \\
167 \\
210 \\
245 \\
200 \\
242 \\
204 \\
180 \\
227 \\
153 \\
176 \\
213 \\
222 \\
300 \\
287 \\
385 \\
233 \\
245 \\
276\end{array}$ & $\begin{array}{l}15 \\
34 \\
89 \\
88 \\
83 \\
81 \\
67 \\
64 \\
61 \\
37 \\
24 \\
32 \\
31 \\
19 \\
17 \\
26 \\
31 \\
22 \\
30 \\
36 \\
21 \\
40 \\
10 \\
21 \\
26 \\
25 \\
29 \\
32 \\
45 \\
23 \\
40\end{array}$ & $\begin{array}{l}293 \\
232 \\
402 \\
390 \\
451 \\
422 \\
264 \\
249 \\
301 \\
251 \\
254 \\
264 \\
229 \\
197 \\
167 \\
198 \\
230 \\
219 \\
217 \\
283 \\
256 \\
219 \\
247 \\
231 \\
186 \\
194 \\
235 \\
274 \\
188 \\
243 \\
267\end{array}$ & $\begin{array}{r}12 \\
9 \\
32 \\
38 \\
25 \\
48 \\
15 \\
19 \\
11 \\
11 \\
1 \\
4 \\
3 \\
10 \\
55 \\
70 \\
11 \\
12 \\
1 \\
13 \\
8 \\
11 \\
4 \\
6 \\
7 \\
12 \\
11 \\
13 \\
59 \\
16 \\
5\end{array}$ & $\begin{array}{l}39 \\
22 \\
41 \\
39 \\
24 \\
34 \\
34 \\
27 \\
29 \\
34 \\
25 \\
19 \\
23 \\
19 \\
31 \\
47 \\
24 \\
23 \\
13 \\
32 \\
39 \\
12 \\
25 \\
26 \\
18 \\
28 \\
37 \\
52 \\
32 \\
31 \\
22\end{array}$ & $\begin{array}{r}3 \\
3 \\
1 \\
1 \\
1 \\
4 \\
8 \\
4 \\
3 \\
4 \\
3 \\
\\
3 \\
2 \\
1 \\
3 \\
1 \\
3 \\
1 \\
2 \\
2 \\
7 \\
1 \\
2 \\
4 \\
1 \\
2 \\
11 \\
1 \\
3\end{array}$ & $\begin{array}{r}9 \\
2 \\
4 \\
3 \\
3 \\
4 \\
\\
1 \\
2 \\
1 \\
9 \\
2 \\
2 \\
4 \\
10 \\
5 \\
6 \\
6 \\
1 \\
6 \\
1\end{array}$ & $\begin{array}{l}1 \\
3 \\
1\end{array}$ & $\begin{array}{l}\text { LPD-4 } \\
\text { Kona-1, Kulani-1 } \\
\text { Kona-2, LPD-3, NER-1 } \\
\text { Kohala-1, LPD-1, Kona-1 } \\
\text { LPD-2, Kona-1 } \\
\text { Kona-2 } \\
\text { LPD-1, NER-1 } \\
\text { Offshore-1, LPD-4 } \\
\text { NER-1 } \\
\text { NER-3 } \\
\text { NER-1 } \\
\text { Kona-1 } \\
\\
\text { Kona-4 } \\
\text { NER-2, Kona-2 } \\
\text { NER-1, Kona-2 } \\
\text { NER-2, Kohala-1 } \\
\text { Kona-2, LPD-9, NER-1 } \\
\text { LPD-1, NER-1, Kona-1 } \\
\text { LPD-1 } \\
\text { LPD-1 } \\
\text { NER-1 } \\
\text { NER-1, LPD-1, Distant-2 } \\
\text { NER-1 } \\
\text { LPD-1, NER-1, Kona-2 } \\
\text { LPD-3, NER-2 }\end{array}$ \\
\hline
\end{tabular}




\begin{tabular}{|c|c|c|c|c|c|c|c|c|c|c|c|c|c|c|}
\hline \multirow{3}{*}{$\begin{array}{l}\text { Date } \\
(1976)\end{array}$} & \multirow{2}{*}{\multicolumn{3}{|c|}{$\begin{array}{c}\text { Tremor } \\
(\mathrm{m}=\text { minutes } \\
\mathrm{h}=\text { hours })\end{array}$}} & \multicolumn{11}{|c|}{ Earthquakes } \\
\hline & & & & \multicolumn{3}{|c|}{ Kilauea Summit } & \multicolumn{4}{|c|}{ Kilauea Flank } & \multicolumn{2}{|c|}{ Mauna Loa } & \multirow[b]{2}{*}{$\begin{array}{l}\text { Off- } \\
\text { shore } \\
\text { PPL }\end{array}$} & \multirow[b]{2}{*}{$\begin{array}{c}\text { Remarks and Events } \\
\text { of Interest }\end{array}$} \\
\hline & Deep & $\begin{array}{l}\text { Inter- } \\
\text { mediate }\end{array}$ & Shallow & $30 \mathrm{KM}$ & $\mathrm{L}-\mathrm{P}$ & $\mathrm{S}-\mathrm{P}$ & $\begin{array}{l}\text { SW Rift } \\
\text { and } \\
\text { Kaoiki }\end{array}$ & $\begin{array}{l}\text { Upper } \\
\text { East } \\
\text { Rift }\end{array}$ & Koae & $\begin{array}{l}\text { Lower } \\
\text { East } \\
\text { Rift }\end{array}$ & $\mathrm{L}-\mathrm{P}$ & $S-P$ & & \\
\hline $\begin{array}{r}1 \\
2 \\
3 \\
4 \\
5 \\
6 \\
7 \\
8 \\
9 \\
10 \\
11 \\
12 \\
13 \\
14 \\
15 \\
16 \\
17 \\
18 \\
19 \\
20 \\
21 \\
22 \\
23 \\
\\
24 \\
25 \\
26 \\
27 \\
28 \\
29 \\
30\end{array}$ & $\begin{array}{l}19 \mathrm{~m} \\
28 \mathrm{~m}\end{array}$ & $\begin{array}{l}7 \mathrm{~m} \\
3 \mathrm{~m} 1 / \\
\\
5 \mathrm{~m} \\
80 \mathrm{~m} 1 / \\
24 \mathrm{~m} \\
\\
5 \mathrm{~m} / \\
14 \mathrm{~m} 1 / \\
20 \mathrm{~m} \\
5 \mathrm{~m} \\
\\
7 \mathrm{~m} \\
3 \mathrm{~m} 1 /\end{array}$ & & $\begin{array}{l}4 \\
1 \\
2 \\
2\end{array}$ & $\begin{array}{r}27 \\
21 \\
20 \\
20 \\
24 \\
3 \\
3 \\
44 \\
21 \\
20 \\
9 \\
1 \\
2 \\
34 \\
5 \\
9 \\
23 \\
28 \\
39 \\
32 \\
62 \\
51 \\
92 \\
9 \\
22 \\
28 \\
27 \\
14 \\
13\end{array}$ & $\begin{array}{l}346 \\
285 \\
302 \\
324 \\
256 \\
398 \\
349 \\
374 \\
397 \\
352 \\
321 \\
317 \\
549 \\
350 \\
322 \\
384 \\
468 \\
425 \\
370 \\
374 \\
419 \\
385 \\
389 \\
343 \\
354 \\
371 \\
364 \\
356 \\
388 \\
379\end{array}$ & $\begin{array}{l}45 \\
32 \\
34 \\
26 \\
45 \\
33 \\
24 \\
41 \\
17 \\
23 \\
21 \\
15 \\
23 \\
21 \\
24 \\
14 \\
15 \\
34 \\
36 \\
30 \\
19 \\
32 \\
33\end{array}$ & $\begin{array}{l}325 \\
307 \\
187 \\
154 \\
135 \\
189 \\
211 \\
224 \\
277 \\
259 \\
208 \\
155 \\
161 \\
260 \\
303 \\
181 \\
173 \\
180 \\
127 \\
132 \\
154 \\
166 \\
176 \\
144 \\
186 \\
194 \\
203 \\
194 \\
159 \\
283\end{array}$ & $\begin{array}{r}11 \\
34 \\
18 \\
8 \\
14 \\
11 \\
12 \\
21 \\
11 \\
19 \\
9 \\
50 \\
42 \\
4 \\
1 \\
3 \\
5 \\
9 \\
46 \\
31 \\
4 \\
9 \\
43 \\
\\
10 \\
10 \\
9 \\
5 \\
6 \\
1 \\
3\end{array}$ & $\begin{array}{l}26 \\
32 \\
23 \\
28 \\
41 \\
39 \\
38 \\
36 \\
41 \\
51 \\
27 \\
30 \\
28 \\
27 \\
30 \\
29 \\
39 \\
41 \\
34 \\
41 \\
28 \\
29 \\
32 \\
38 \\
26 \\
34 \\
36 \\
33 \\
21 \\
27\end{array}$ & $\begin{array}{r}15 \\
3 \\
2 \\
2 \\
4 \\
2 \\
3 \\
10 \\
2 \\
23 \\
7 \\
1 \\
2 \\
\\
1 \\
\\
45 \\
7 \\
347 \\
306 \\
223 \\
152 \\
67 \\
36 \\
17 \\
24 \\
7 \\
8 \\
14 \\
8\end{array}$ & $\begin{array}{r}2 \\
2 \\
4 \\
2 \\
8 \\
5 \\
8 \\
10 \\
9 \\
16 \\
4 \\
8 \\
6 \\
5 \\
5 \\
1 \\
2 \\
7 \\
21 \\
4 \\
1 \\
39 \\
27 \\
18 \\
46 \\
17 \\
17 \\
19 \\
17 \\
12\end{array}$ & $\begin{array}{l}1 \\
2 \\
1\end{array}$ & $\begin{array}{l}\text { NER-1, LPD-8 } \\
\text { Kona-2 } \\
\text { LPD-1, NER-2 } \\
\text { NER-1 } \\
\text { NER-2, Kona-2 } \\
\text { NER-1, Kona-2 } \\
\text { LPD-3, NER-1 } \\
\text { Distant-1, Kona-1 } \\
\text { LPD-1 } \\
\text { LPD-2 } \\
\text { Mauna Kea-1 } \\
\text { Maui-1, LPD-1 } \\
\text { Maui-1, NER-1 } \\
\text { NER-1 } \\
\text { LPD-2, Kona-1 } \\
\text { Kona-1 } \\
\text { NER-1 } \\
\text { NER-5 } \\
\text { NER-1 } \\
\text { Kona-2, LPD-2, NER-4 } \\
\\
\text { NER-5, Kona-2, LPD-2 } \\
\text { NER-1, Kona-1 } \\
\text { NER-4, Kona-4 } \\
\text { LPD-1, NER-2, Kona-1 } \\
\text { NER-2 } \\
\text { Maui-1, NER-2 } \\
\text { NER-1 }\end{array}$ \\
\hline
\end{tabular}

1/MOK-tremor beneath Mauna Loa Volcano 


\begin{tabular}{|c|c|c|c|c|c|c|c|c|c|c|c|c|c|c|}
\hline \multirow{3}{*}{$\begin{array}{l}\text { Date } \\
(1976)\end{array}$} & \multirow{2}{*}{\multicolumn{3}{|c|}{$\begin{array}{c}\text { Tremor } \\
(\mathrm{m}=\text { minutes } \\
\mathrm{h}=\text { hours })\end{array}$}} & \multicolumn{11}{|c|}{ Earthquakes } \\
\hline & & & & \multicolumn{3}{|c|}{ Kilauea Summit } & \multicolumn{4}{|c|}{ Kilauea Flank } & \multicolumn{2}{|c|}{ Mauna Loa } & \multirow[b]{2}{*}{$\begin{array}{l}\text { Off- } \\
\text { shore } \\
\text { PPI }\end{array}$} & \multirow[b]{2}{*}{$\begin{array}{c}\text { Remarks and Events } \\
\text { of Interest }\end{array}$} \\
\hline & Deep & $\begin{array}{l}\text { Inter- } \\
\text { mediate }\end{array}$ & Shallow & $30 \mathrm{KM}$ & $I-P$ & $S-P$ & $\begin{array}{l}\text { SW Rift } \\
\text { and } \\
\text { Kaoiki }\end{array}$ & $\begin{array}{l}\text { Upper } \\
\text { East } \\
\text { Rift }\end{array}$ & Koae & $\begin{array}{l}\text { Lower } \\
\text { East } \\
\text { Rift }\end{array}$ & $\mathrm{L}-\mathrm{P}$ & $S-P$ & & \\
\hline $\begin{array}{r}1 \\
2 \\
3 \\
4 \\
5 \\
6 \\
7 \\
8 \\
9 \\
10 \\
11 \\
12 \\
13 \\
14 \\
15 \\
16 \\
17 \\
18 \\
19 \\
20 \\
21 \\
22 \\
23 \\
24 \\
25 \\
26 \\
27 \\
28 \\
29 \\
30 \\
31\end{array}$ & $\begin{array}{l}38 \mathrm{~m} \\
30 \mathrm{~m} \\
23 \mathrm{~m} \\
20 \mathrm{~m} \\
31 \mathrm{~m} \\
60 \mathrm{~m} 1 /\end{array}$ & $\begin{array}{l}9 \mathrm{~m} \\
7 \mathrm{~m} \\
5 \mathrm{~m} \\
3 \mathrm{~m}=1 / \\
5 \mathrm{~m} \frac{1}{1 /} \\
8 \mathrm{~m}=\end{array}$ & & $\begin{array}{l}2 \\
1 \\
1 \\
3\end{array}$ & $\begin{array}{r}13 \\
19 \\
3 \\
4 \\
13 \\
18 \\
23 \\
14 \\
17 \\
12 \\
19 \\
19 \\
23 \\
42 \\
12 \\
4 \\
4 \\
3 \\
20 \\
18 \\
19 \\
7 \\
24 \\
32 \\
19 \\
9 \\
20 \\
5 \\
20\end{array}$ & $\begin{array}{l}406 \\
345 \\
396 \\
431 \\
332 \\
403 \\
383 \\
493 \\
467 \\
272 \\
167 \\
333 \\
486 \\
319 \\
501 \\
767 \\
358 \\
161 \\
303 \\
372 \\
422 \\
338 \\
296 \\
318 \\
311 \\
355 \\
361 \\
481 \\
296 \\
519 \\
414\end{array}$ & $\begin{array}{r}43 \\
30 \\
28 \\
22 \\
32 \\
33 \\
30 \\
26 \\
38 \\
44 \\
42 \\
48 \\
20 \\
48 \\
114 \\
20 \\
64 \\
26 \\
30 \\
39 \\
24 \\
49 \\
21 \\
21 \\
35 \\
38 \\
25 \\
28 \\
26 \\
19 \\
5\end{array}$ & $\begin{array}{l}274 \\
275 \\
209 \\
220 \\
273 \\
203 \\
207 \\
185 \\
193 \\
176 \\
143 \\
245 \\
217 \\
266 \\
474 \\
147 \\
496 \\
211 \\
146 \\
270 \\
259 \\
232 \\
264 \\
258 \\
330 \\
377 \\
299 \\
338 \\
355 \\
168 \\
137\end{array}$ & $\begin{array}{r}2 \\
15 \\
35 \\
37 \\
6 \\
2 \\
7 \\
1 \\
3 \\
15 \\
37 \\
2 \\
32 \\
15 \\
\\
35 \\
10 \\
4 \\
5 \\
7 \\
11 \\
1 \\
8 \\
5 \\
9 \\
9 \\
18 \\
21 \\
18 \\
77 \\
37\end{array}$ & $\begin{array}{r}45 \\
41 \\
38 \\
26 \\
53 \\
29 \\
30 \\
29 \\
26 \\
25 \\
38 \\
17 \\
26 \\
11 \\
72 \\
23 \\
20 \\
8 \\
57 \\
10 \\
21 \\
11 \\
20 \\
24 \\
20 \\
24 \\
12 \\
19 \\
21 \\
4 \\
9\end{array}$ & $\begin{array}{r}2 \\
11 \\
1 \\
2 \\
5 \\
6 \\
3 \\
4 \\
6 \\
2 \\
13 \\
7 \\
\\
7 \\
13 \\
\\
5 \\
3 \\
8 \\
4 \\
8 \\
5 \\
10 \\
14 \\
3 \\
4 \\
8 \\
8 \\
15 \\
8\end{array}$ & $\begin{array}{r}9 \\
2 \\
4 \\
4 \\
4 \\
2 \\
2 \\
10 \\
5 \\
4 \\
7 \\
10 \\
7 \\
5 \\
15 \\
9 \\
3 \\
4 \\
21 \\
8 \\
1 \\
17 \\
1 \\
1 \\
1 \\
4 \\
2 \\
2 \\
7 \\
7\end{array}$ & $\begin{array}{l}3 \\
1 \\
1\end{array}$ & $\begin{array}{l}\text { NER-3, LPD-5, Kona-1 } \\
\text { NER-2, Kona-1 } \\
\text { NER-3 } \\
\text { Kona-1 } \\
\text { Kona-1 } \\
\text { NER-2 } \\
\text { Kona-1, NER-1 } \\
\text { LPD-4 } \\
\text { Mauna Kea-2, NER-4 } \\
\text { Kulani-2, LPD-2 } \\
\text { NER-2 } \\
\text { LPD-7 } \\
\text { NER-2, Kulani-1 } \\
\text { NER-1 } \\
\text { NER-1 } \\
\text { Kona-1 } \\
\text { NER-2, Kona-1 } \\
\text { NER-1, Kona-2 } \\
\text { NER-1 } \\
\text { LPD-7 } \\
\text { NER-1, } \\
\text { Nen- } \\
\text { Nen- }\end{array}$ \\
\hline
\end{tabular}

1/ MOK-tremor beneath Mauna Loa Volcano 
CONTENTS

Page

Introduction. . . . . . . . 1

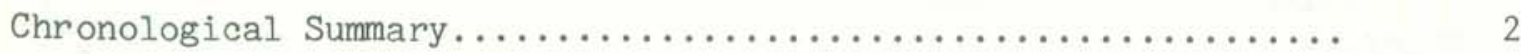

Seismic Instrumentation. ............................

Figure 1 Map of Hawaii showing geographic and geologic

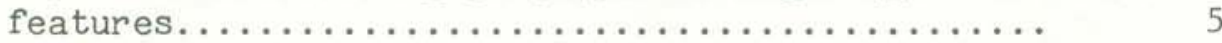

Figure 2 Map of Hawaii showing active seismic stations..... 6

Table 1 Seismic stations on Hawaii operated by the USGS... 7

Table 2 Seismic instrumentation types in use by HVO...... 8

Figure 3 System response curve of the four basic seismograph types in use by HVO............... 9

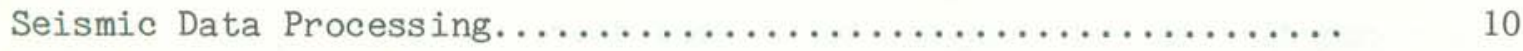

Figure 4 Relationship between duration time and local

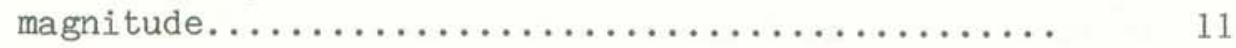

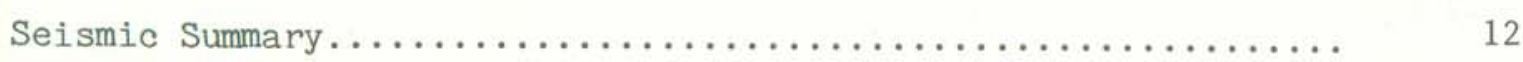

Table 3 Number of earthquakes and minutes of tremor recorded on seismographs around Kilauea..........

Figure 5 Epicenter map of Hawaii showing only magnitudes

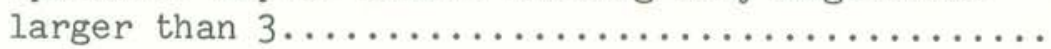

Figure 6 Epicenter map of Hawaii showing all earthquakes...

Figure 7 Epicenter map of the Kilauea/Mauna Loa region showing only magnitudes larger than $3 \ldots \ldots \ldots \ldots$.

Figure 8 Epicenter map of the Kilauea/Mauna Loa region

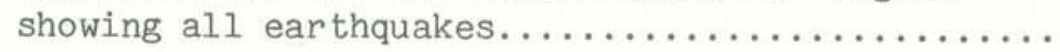

Figure 9 Map of Hawaii showing geographic codes used for classifying earthquakes by region.............

Table 4 Coordinates of named regions used for classifying

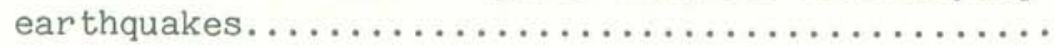

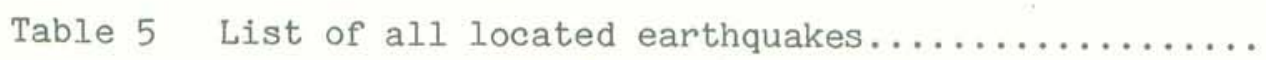




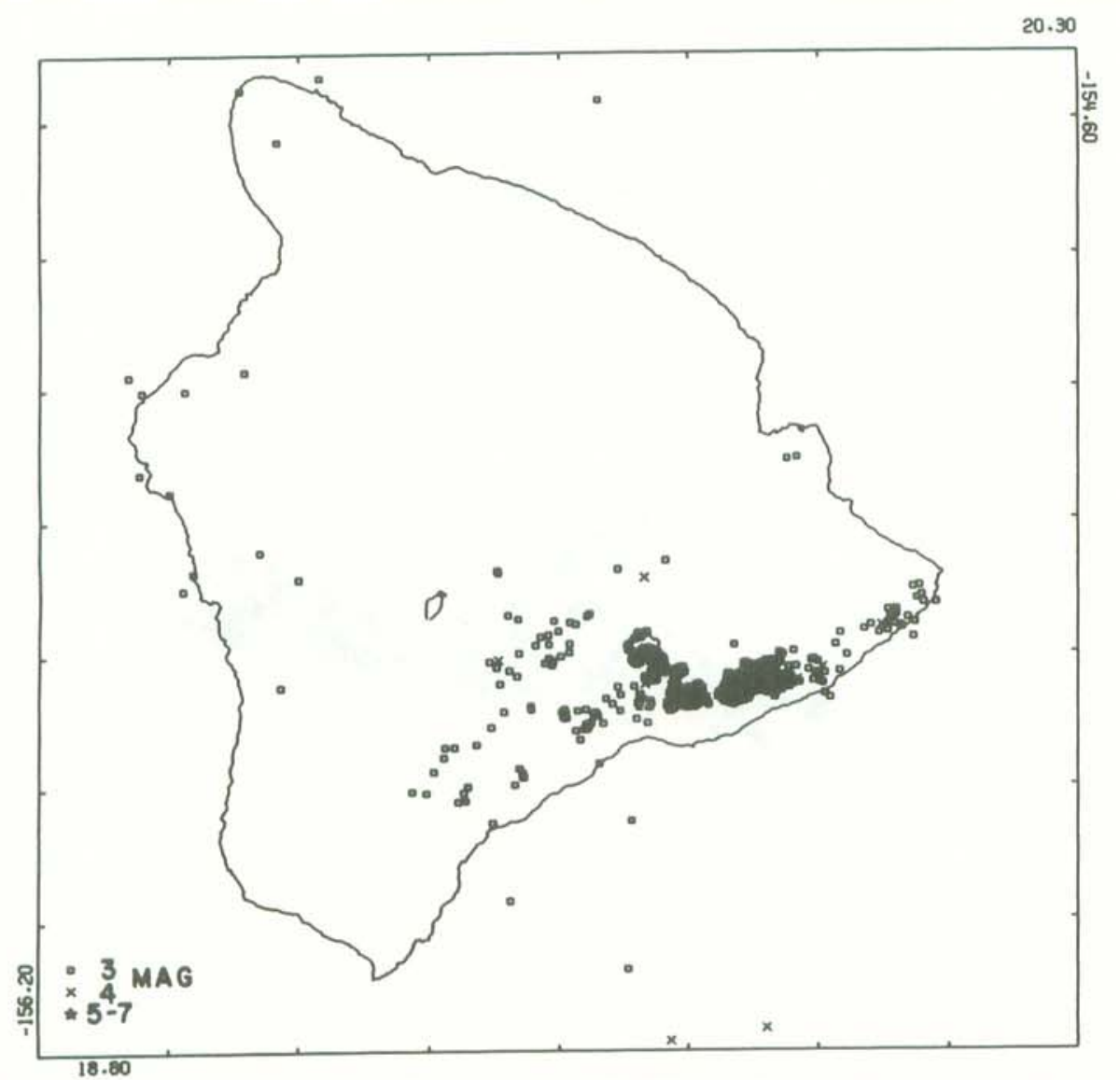

Figure 5 Epicenter plot of magnitude 3 and above earthquakes for the year 1976.

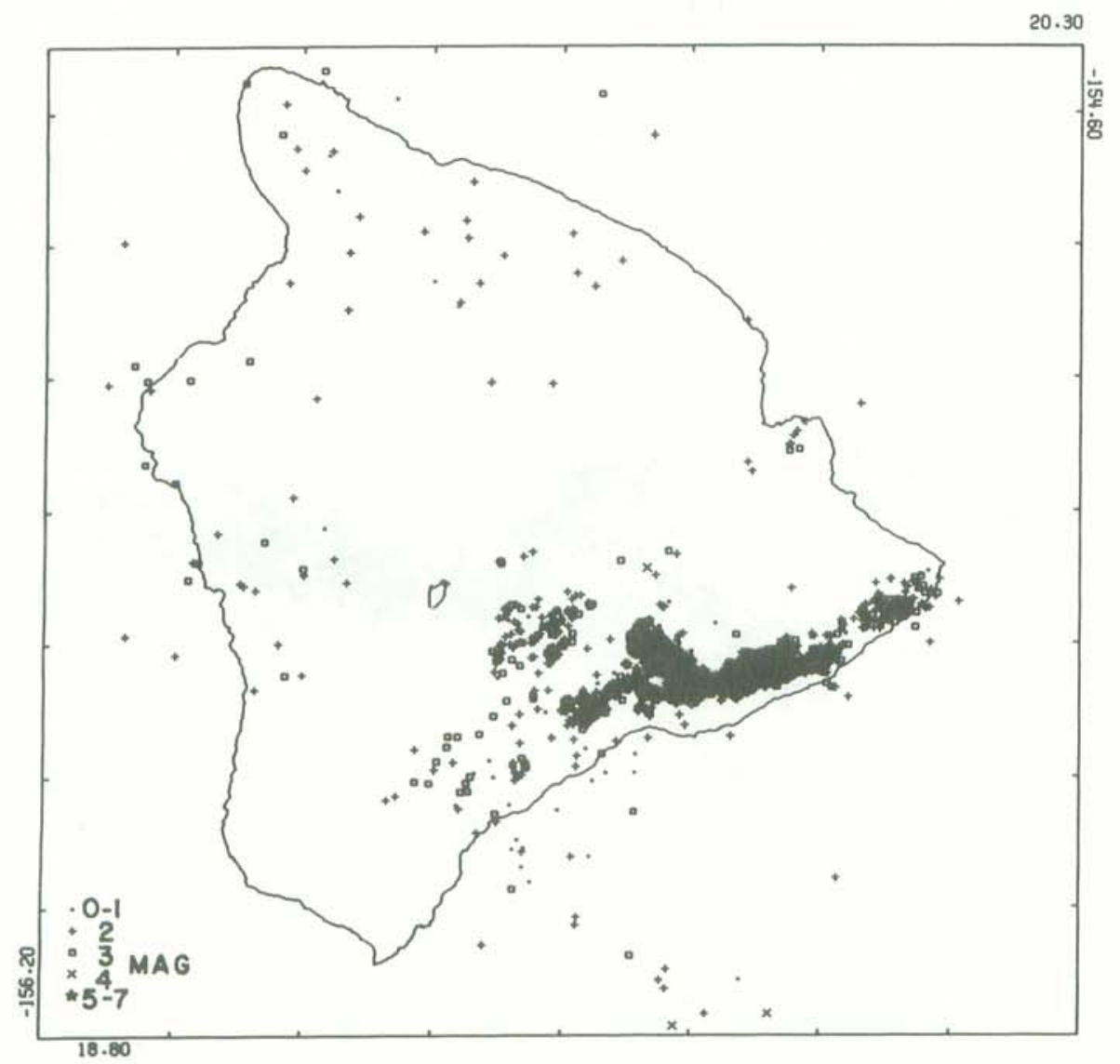

Figure 6. Epicenter plot of all events located for the year 1976. 
19.60

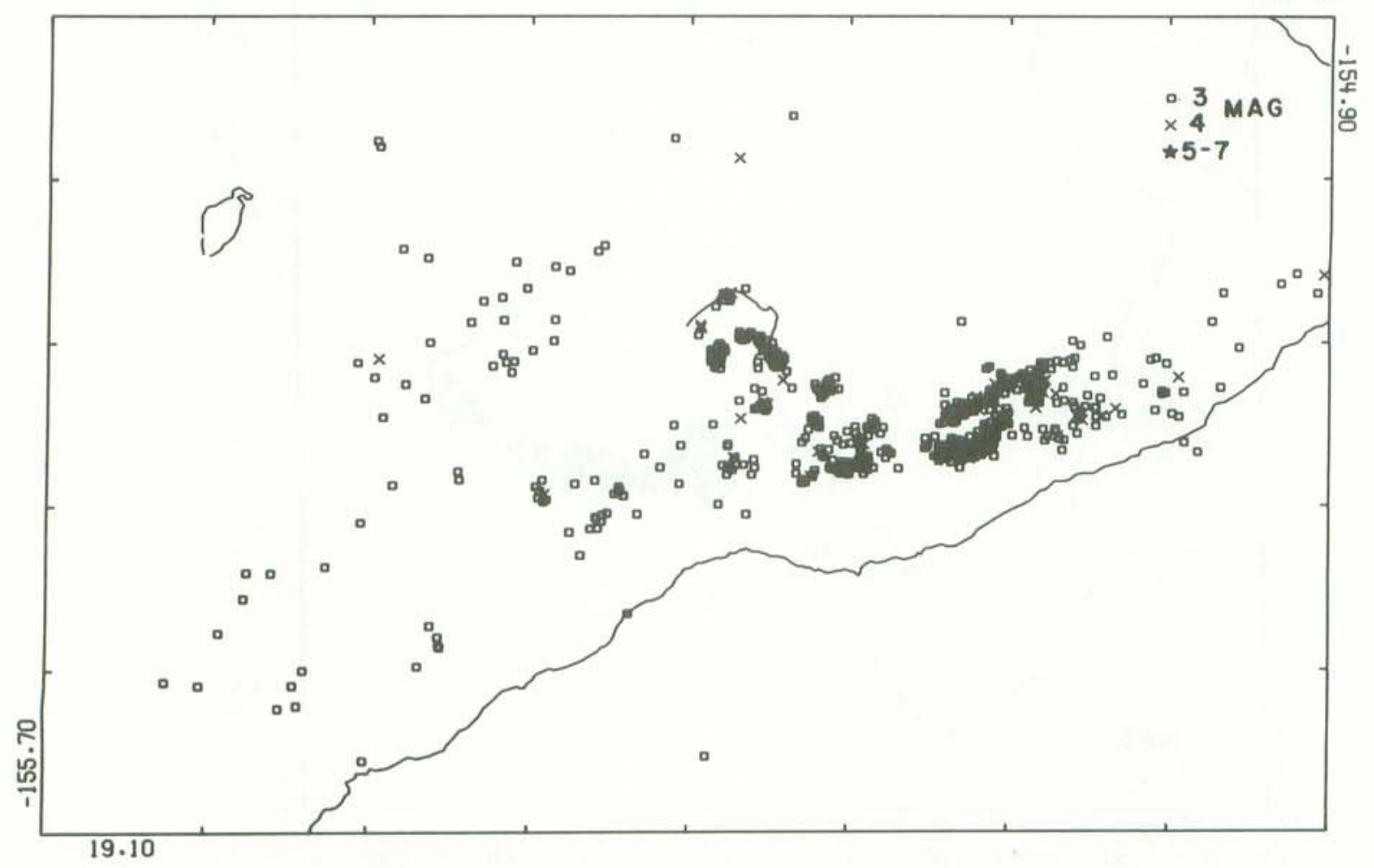

Figure 7. Epicenter plot of Mauna Loa and Kilauea earthquakes magnitude 3 and above for the year 1976.

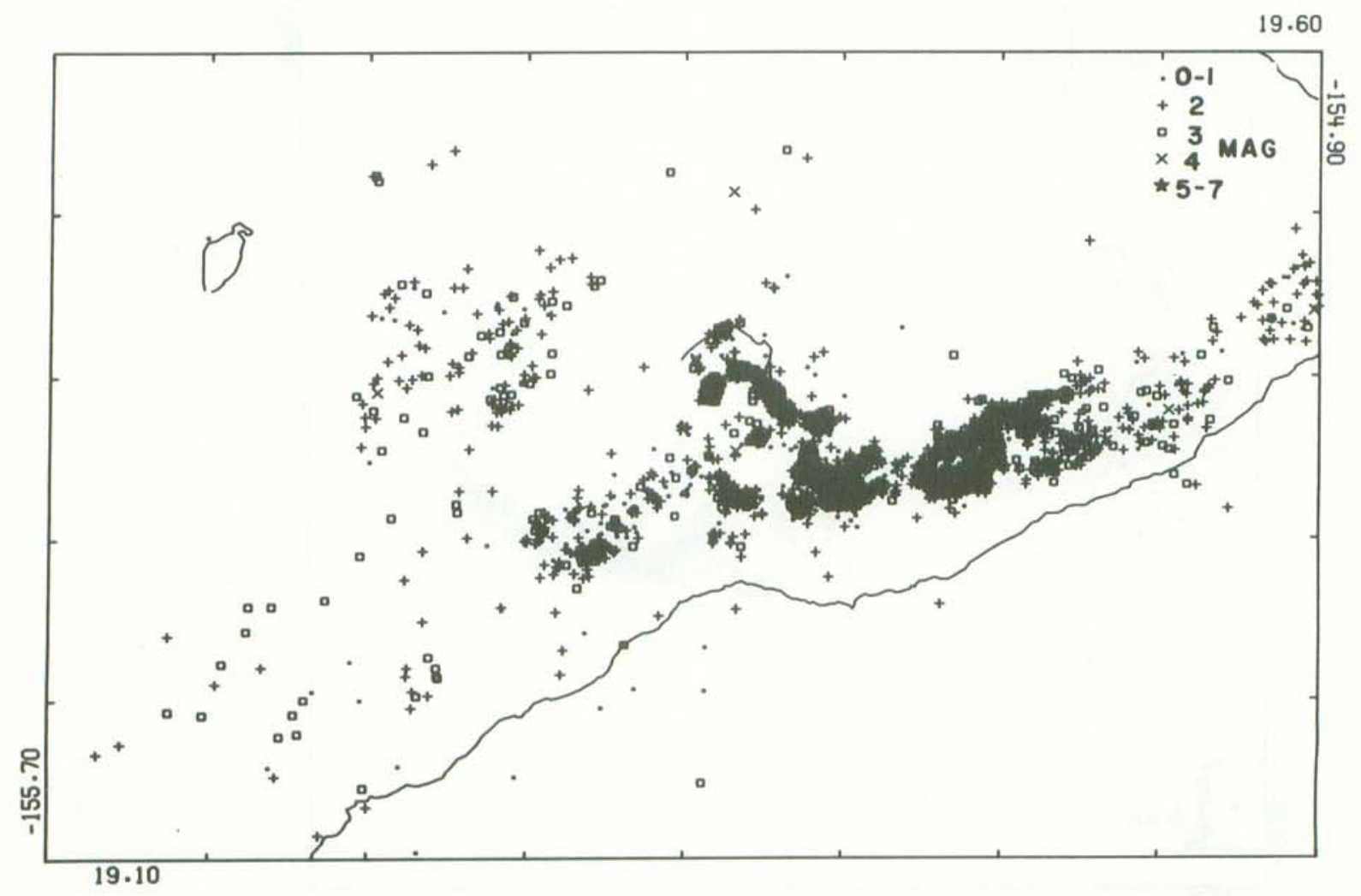

Figure 8. Epicenter plot of all Mauna Loa and Kilauea earthquakes located for the year 1976. 


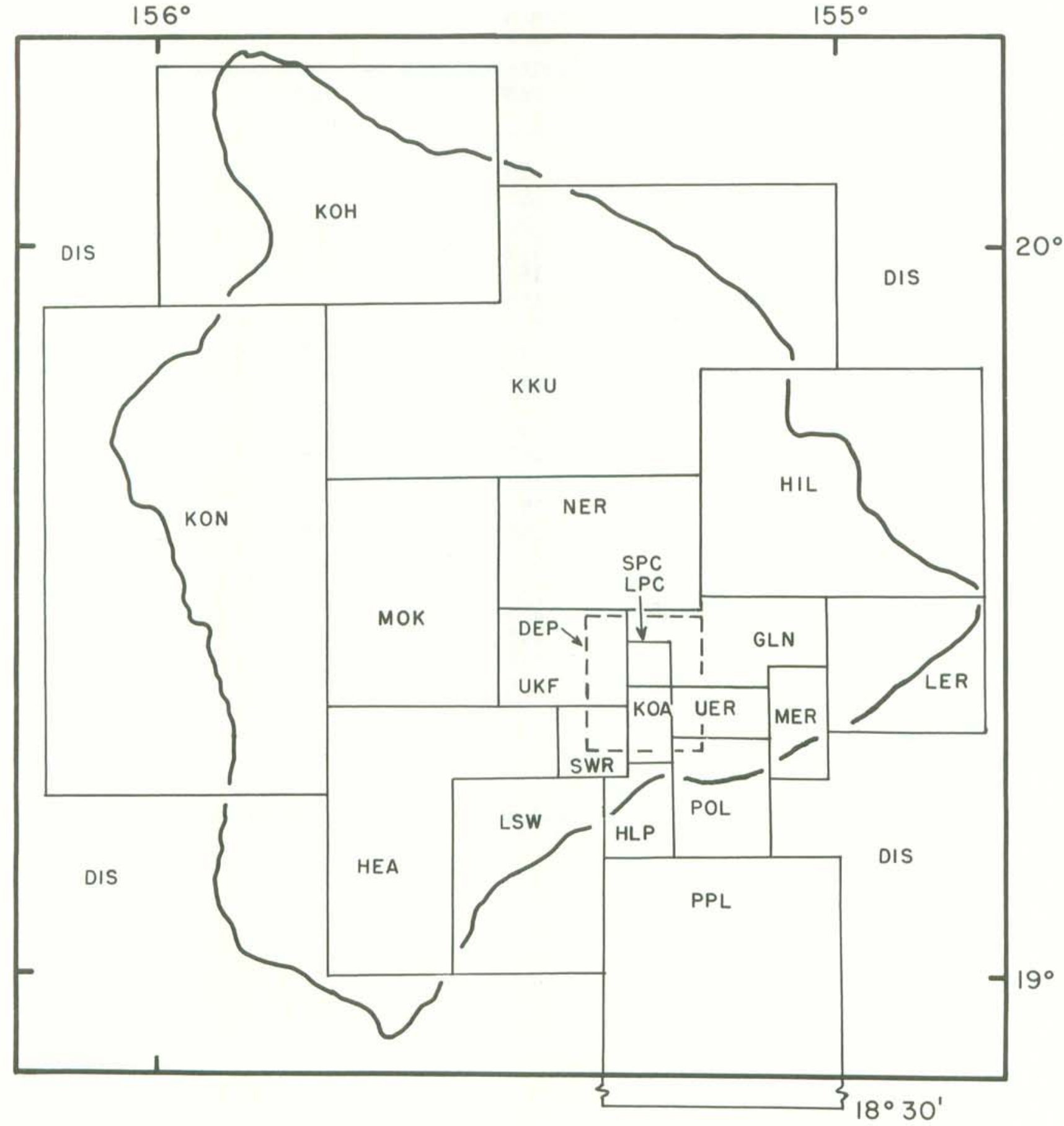

Figure 9. Map indicating limits of areas for specific mnemonic codes used in the remarks column of the earthquake summary. DEP is a code applied to earthquakes with depths greater than 13 kilometres in the Kilauea region. SPC is used for depths between 0 and $6 \mathrm{~km}$, and LPC is used between 6 and 13 $\mathrm{km}$. 
Table 4. Coordinates of named regions.

\begin{tabular}{|c|c|c|c|c|c|c|c|c|c|}
\hline \multirow[t]{3}{*}{ Name } & \multicolumn{9}{|c|}{ ( } \\
\hline & \multicolumn{2}{|c|}{ NORTH } & \multicolumn{2}{|c|}{ SOUTH } & \multicolumn{2}{|c|}{ EAST } & \multicolumn{2}{|c|}{ WEST } & \multirow{2}{*}{$\begin{array}{c}\text { DEPTHS } \\
0 \\
\end{array}$} \\
\hline & D & M & D & M & D & M & D & M & \\
\hline SPC & 19 & 27 & 19 & 23 & 155 & 15 & 155 & 19 & $0-6$ \\
\hline LPC & 19 & 27 & 19 & 23 & 155 & 15 & 155 & 19 & $6-13$ \\
\hline DEP & 19 & 29 & 19 & 18 & 155 & 22 & 155 & 22 & $13-70$ \\
\hline UER & 19 & 23 & 19 & 19 & 155 & 6 & 155 & 15 & \\
\hline KOA & 19 & 23 & 19 & 17 & 155 & 15 & 155 & 19 & \\
\hline SWR & 19 & 22 & 19 & 16 & 155 & 19 & 155 & 25 & \\
\hline UKF & 19 & 29 & 19 & 22 & 155 & 19 & 155 & 30 & \\
\hline MER & 19 & 25 & 19 & 16 & 155 & 1 & 155 & 6 & \\
\hline LER & 19 & 31 & 19 & 20 & 154 & 47 & 155 & 1 & \\
\hline POL & 19 & 19 & 19 & 10 & 155 & 6 & 155 & 15 & \\
\hline LSW & 19 & 16 & 19 & 0 & 155 & 21 & 155 & 34 & \\
\hline PPL & 19 & 10 & 18 & 30 & 155 & 0 & 155 & 21 & \\
\hline HLP & 19 & 17 & 19 & 10 & 155 & 15 & 155 & 21 & \\
\hline MOK & 19 & 40 & 19 & 22 & 155 & 30 & 155 & 45 & \\
\hline GLN & 19 & 31 & 19 & 23 & 155 & 1 & 155 & 19 & \\
\hline KON & 19 & 55 & 19 & 15 & 155 & 45 & 156 & 10 & \\
\hline HEA & 19 & 22 & 19 & 0 & 155 & 25 & 155 & 45 & \\
\hline $\mathrm{KOH}$ & 20 & 15 & 19 & 55 & 155 & 30 & 156 & 0 & \\
\hline NER & 19 & 40 & 19 & 29 & 155 & 12 & 155 & 30 & \\
\hline HIL & 19 & 50 & 19 & 31 & 154 & 47 & 155 & 12 & \\
\hline KKU & 20 & 5 & 19 & 40 & 155 & 0 & 155 & 45 & \\
\hline DIS & \multicolumn{9}{|c|}{ EVERYPLACE ELSE } \\
\hline BLS & \multicolumn{9}{|c|}{ QUARRY BLAST } \\
\hline
\end{tabular}

When coordinates imply an overlap, precedence is given as shown in Figure 9. 
Table 5 is a chronological listing of successfully located earthquakes. For each event the following data are presented:

Origin time in Hawaiian Standard Time: date, hour (HR), minute (MN), and second (SEC).

Epicenter in degrees and minutes of North latitude (LAT N) and west longitude (LON W).

DEPTH - Depth of focus in $\mathrm{km}$.

AMP MAG - Amplitude magnitude, if determined.

DUR MAG - Duration magnitude, if determined.

NR - Number of arrivals ( $P$ or $S$ ) used for solution.

NS - Number of S arrivals used for solution.

GAP DEG - Largest azimuthal separation in degrees between stations.

RMS SEC - Root mean square error of time residuals in sec.

$$
\mathrm{RMS}=\left(\sum \mathrm{R}_{i}^{2} / \mathrm{NR}\right)^{1 / 2}
$$

MIN DIS - Epicentral distance in $\mathrm{km}$ to the third nearest station.

ERH $\mathrm{km}$ - Standara error of the epicenter in $\mathrm{km}$.

ERZ $\mathrm{km}$ - Standrad error of depth of focus in $\mathrm{km}$.

REMK - Remarks, three letter code for geographic location of event. See Figure 9 for location of mnemonic code.

Table 5 lists all events located during 1976. Table 6 lists only events of magnitude 3.5 or larger. 


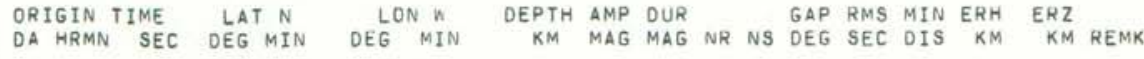

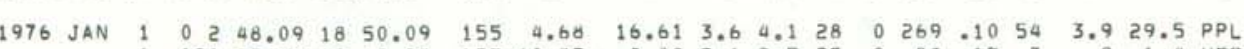

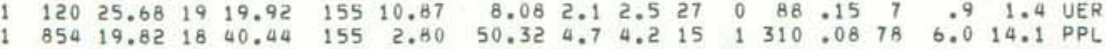

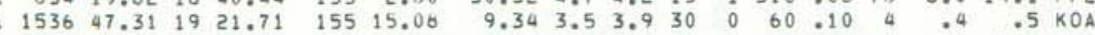

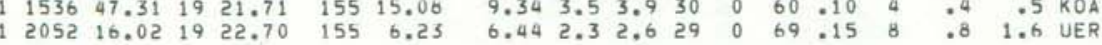

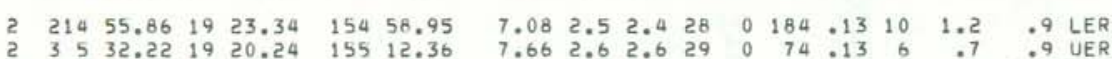

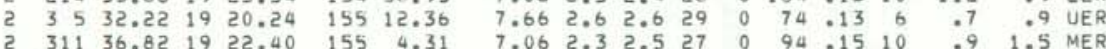

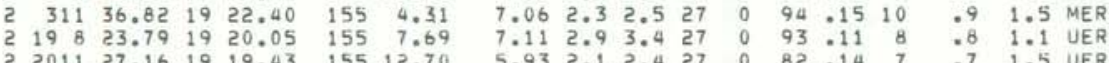
$\begin{array}{llll}20 & 19 & 19.43 \quad 155 \quad 12.70\end{array}$ $\begin{array}{rrrrrrr}2 & 2018 & 21.43 & 19 & 22.31 & 155 & 7.00 \\ 3 & 137 & 13.34 & 19 & 20.42 & 155 & 19.29\end{array}$

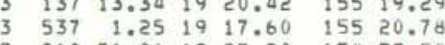
$31352 \quad 3.3419,17.38 \quad 15522.00$

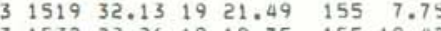
3 1532 $22.261919 .75 \quad 15519.42$ 3.

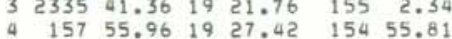

$\begin{array}{llllll}451 & 7.70 & 19 & 22.45 & 155 & 1.51\end{array}$ 4 $52139.411920 .31 \quad 155 \quad 8.67$ $\begin{array}{lllllll}4 & 557 & 26.71 & 19 & 24.23 & 155 & 16.10 \\ 4 & 620 & 50.96 & 19 & 28.56 & 154 & 52.23\end{array}$

$4 \quad 727 \quad 58.901922 .60 \quad 155 \quad 5.06$

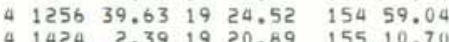
$4.14242 .391920 .89 \quad 155 \quad 10.70$

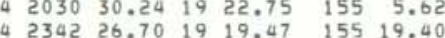

$\begin{array}{lllllll}5 & 634 & 43.45 & 19 & 19.67 & 155 & 19.29\end{array}$

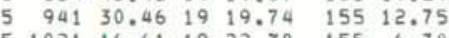

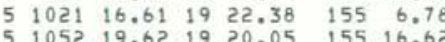

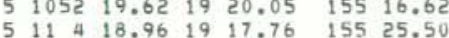
$\begin{array}{lllllll}5 & 1111 & 15.10 & 19 & 20.44 & 155 & 8.78\end{array}$

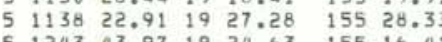

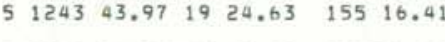

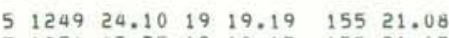
$\begin{array}{lllllll}1254 & 47.75 & 19 & 18.17 & 155 & 21.17\end{array}$ 5
5 $1327 \quad 22.741920 .08 \quad 155 \quad 16.33$ $\begin{array}{lrrrrrr}5 & 1353 & 40.46 & 19 & 21.39 & 155 & 10.91 \\ 5 & 1417 & 59.77 & 19 & 21.97 & 155 & 6.41\end{array}$ $\begin{array}{lllllll}5 & 1421 & 40.27 & 19 & 19.95 & 155 & 8.44\end{array}$

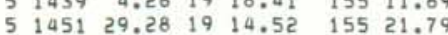

HVO EARTHQUAKE SUMMARY LIST

PAGE ORIGIN TIME LAT N LON W DEPTH AMP DUR N GAP RMS MIN ERH ERZ REMK

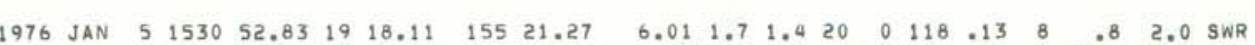
$\begin{array}{lllllll}5 & 1625 & 59.31 & 19 & 17.82 & 155 & 21.31\end{array}$ 55162932.011917 .8915521 .54

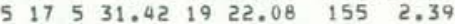

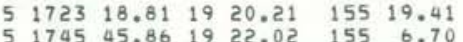

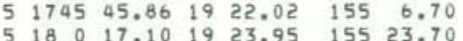

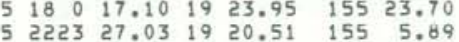
5252327.03 19 $20.51 \quad 155 \quad 5.89$

$\begin{array}{lrlllll}5 & 2317 & 44.84 & 19 & 19.54 & 155 & 12.72\end{array}$ $\begin{array}{lllllll}6 & 043 & 37.58 & 19 & 18.71 & 155 & 23.77 \\ 6 & 047 & 2.75 & 19 & 19.24 & 155 & 12.94\end{array}$ $\begin{array}{rrrrrrr}6 & 047 & 2.75 & 19 & 19.24 & 155 & 12.94 \\ 6 & 054 & 34.51 & 19 & 12.39 & 155 & 19.91\end{array}$

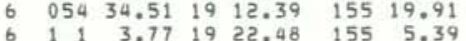

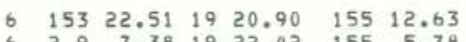

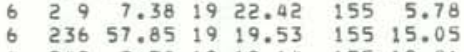
$6 \quad 242 \quad 2.581919 .14 \quad 15519.86$

$\begin{array}{lllllll}6 & 258 & 12.78 & 19 & 20.02 \quad 155 & 16.71\end{array}$ $6 \quad 316 \quad 6.23 \quad 19 \quad 19.00 \quad 155 \quad 13.36$ $\begin{array}{lllllll}6 & 323 & 27.33 & 19 & 20.53 & 155 & 17.78\end{array}$

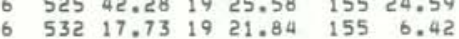

$\begin{array}{lllllll}6 & 626 & 58.37 & 19 & 20.98 & 155 & 11.18\end{array}$ $\begin{array}{lllllll}6 & 719 & 44.42 & 19 & 21.31 & 155 & 19.20\end{array}$ $\begin{array}{lllllll}6 & 724 & 2.05 & 19 & 20.04 & 155 & 11.33\end{array}$ $\begin{array}{lllllll}6 & 745 & 8.98 & 19 & 20.54 & 155 & 9.13 \\ 6 & 752 & 7.19 & 19 & 17.51 & 155 & 20.69\end{array}$

$\begin{array}{lllllll}6 & 758 & 29.20 & 19 & 20.44 & 155 & 10.08\end{array}$ $\begin{array}{llllllll}6 & 8 & 6 & 12.76 & 19 & 21.80 & 155 & 8.35\end{array}$ $\begin{array}{llllll}958 & 33.64 & 19 & 19.13 \quad 155 & 19.05\end{array}$

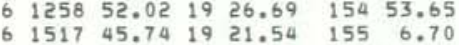

$\begin{array}{lllllll}7 & 016 & 39.36 & 19 & 18.69 & 155 & 20.66\end{array}$ $\begin{array}{llllll}144 & 33.33 & 19 & 27.20 & 154 & 53.21 \\ 952 & 33.40 & 19 & 18.96 & 155 & 23.34\end{array}$

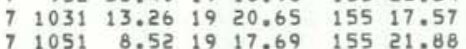

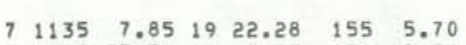
$\begin{array}{lllllll}8 & 222 & 53.36 & 19 & 47.65 & 156 & 2.54 \\ 8 & 817 & 42.30 & 19 & 21.38 & 155 & 13.26\end{array}$ $8 \quad 1925 \quad 7.081920 .22 \quad 15516.55$ $822753.111921 .44 \quad 155 \quad 8.23$
0 $9 \begin{array}{lllllll}9 & 19.37 \quad 19 & 19.61 \quad 155 & 12.61\end{array}$

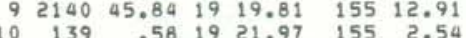

$\begin{array}{llllllllllll}6.01 & 1.7 & 1.4 & 20 & 0 & 118 & .13 & 8 & .8 & 2.0 & \text { SWR } \\ 6.99 & 1.7 & 1.1 & 15 & 0 & 158 & 0.11 & 8 & 1.1 & 2.3 & \text { SWR }\end{array}$

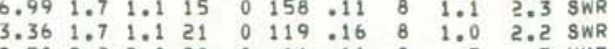

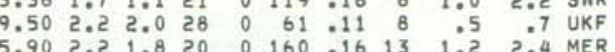

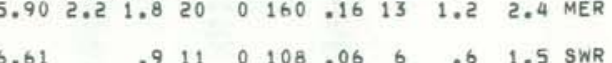
$\begin{array}{lllllllllll}6.61 & & .9 & 11 & 0 & 108 & 0.06 & 6 & .6 & 1.5 & \text { SWR } \\ 6.84 & 1.9 & 2.0 & 21 & 0 & 78 & .15 & 8 & 1.0 & 1.8 & \text { UER }\end{array}$ $\begin{array}{lllllllllll}8.91 & 1.9 & 2.1 & 23 & 0 & 68 & 0.11 & 7 & .6 & 1.2 & \text { UKF }\end{array}$

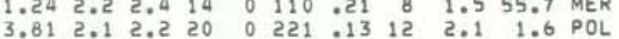

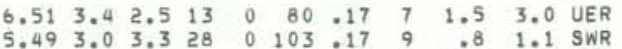
$\begin{array}{lllllllllll}5.49 & 3.0 & 3.3 & 28 & 0 & 103 & .17 & 9 & .8 & 1.1 & \text { SWR } \\ 5.60 & .6 & 2 & 17 & 0 & 81 & .15 & 8 & 1.0 & 2.9 & \text { UER }\end{array}$

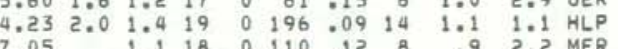

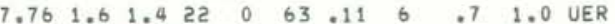

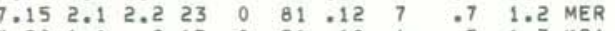
$\begin{array}{lllllllllll}0.5 & 1.6 & 0 & 17 & 0 & 96 & -10 & 6 & .7 & 1.3 & \text { KOA } \\ 0.5 & 1.6 & 7 & 14 & 0 & 120 & .09 & 6 & 0 & 1.3 & \text { SWR }\end{array}$

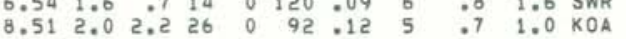
$\begin{array}{lllllllllll}7.06 & 1.5 & .8 & 15 & 0 & 87 & .09 & 5 & .7 & 1.4 & \text { KOA }\end{array}$

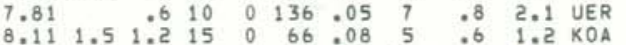

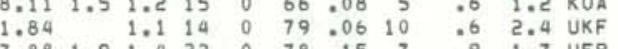

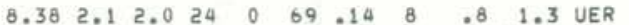
.599 .0 SWR

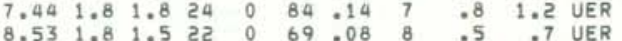

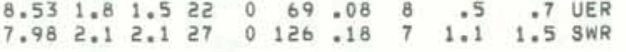
\begin{tabular}{lllllllllll}
7.52 & 1.7 & 1.3 & 22 & 0 & 76 & .14 & 7 & .9 & 1.8 & UER \\
\hline
\end{tabular} $\begin{array}{llllllllll}8.67 & 0.6 & 2.8 & 27 & 0 & 60 & .12 & 8 & 7 & 9\end{array}$

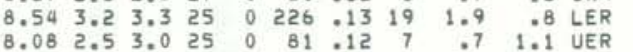
$\begin{array}{llllllllllll}9.64 & 3.7 & 3.7 & 30 & 0 & 106 & .11 & 6 & .7 & .4 & \text { SWR }\end{array}$

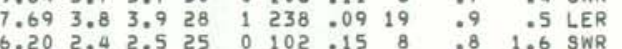
$\begin{array}{llllllllllll}7.37 & 2.3 & 2.7 & 25 & 0 & 57 & .14 & 5 & .7 & 1.0 & \mathrm{KOA}\end{array}$

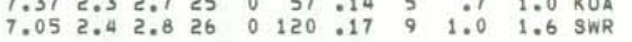
$\begin{array}{lllllllllll}7.89 & 3.1 & 3.2 & 28 & 0 & 81 & .11 & 7 & .6 & 1.0 & \text { MER }\end{array}$

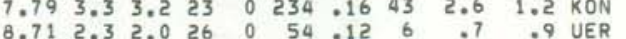

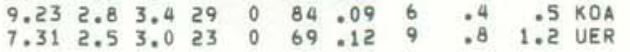
$\begin{array}{lllllllllll}6.58 & 2.3 & 2.8 & 26 & 0 & 80 & .17 & 7 & .9 & 1.8 & 1.8 \text { UER }\end{array}$

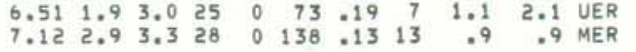


ORIGIN TIME LATN
LON W DEPTH AMP OUR YEAR MON DA HRMN SEC DEG MIN DEG MIN KM MAG MAG NR NS DEG SEC DIS KM KM REMK

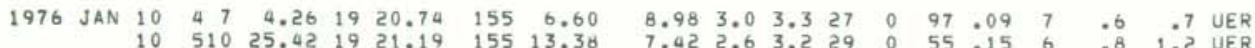

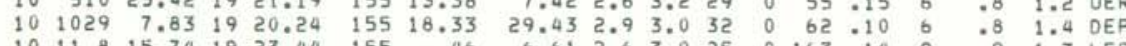

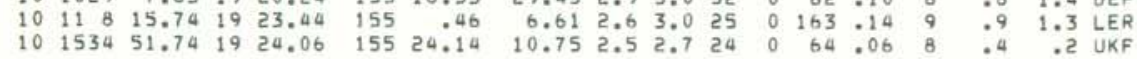
$\begin{array}{llllll}10 & 1652 & 22.99 & 19 & 20.72 \quad 155 \quad 6.85\end{array}$

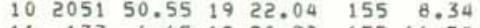
$\begin{array}{lllllll}11 & 133 & 6.65 & 19 & 20.32 & 155 & 16.78\end{array}$

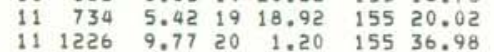

$\begin{array}{llllllll}11 & 14 & 5 & 40.17 & 19 & 30.77 & 155 & 16.17\end{array}$ $\begin{array}{lllllll}12 & 348 & 32.91 & 19 & 21.90 & 155 & 6.67\end{array}$

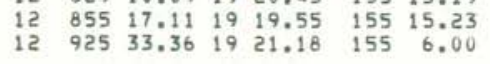
$\begin{array}{lllllll}12 & 938 & 5.95 & 19 & 24.21 & 155 & 15.97\end{array}$

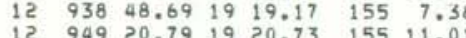
$12 \quad 951 \quad 31.95 \quad 19 \quad 24.13 \quad 155 \quad 15.93$ $121045 \quad 1.261924 .06 \quad 15515.92$

$\begin{array}{lllllll}12 & 1113 & 38.78 & 19 & 21.03 & 155 & 13.43\end{array}$ $\begin{array}{lllllll}12 & 1133 & 11.32 & 19 & 19.87 & 155 & 16.0 \\ 12 & 1241 & 26.15 & 19 & 21.12 & 155 & 7.0\end{array}$

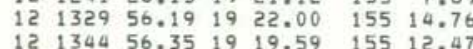

$\begin{array}{lllllll}12 & 1416 & 43.09 & 19 & 19.70 & 155 & 12.6\end{array}$

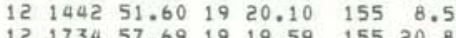

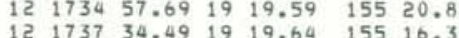
$12 \quad 1746 \quad 24.82 \quad 1920.46 \quad 155 \quad 13.11$

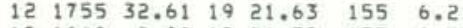
$\begin{array}{lllllll}12 & 1840 & 8.08 & 19 & 21.52 & 155 & 6.7\end{array}$ 12 1950 $16.701927 .96 \quad 15455.00$ 122017 12.11 $1920.61 \quad 155$ 12.26

$\begin{array}{lllllll}12 & 2027 & 14.98 & 19 & 19.78 & 155 & 12.45\end{array}$

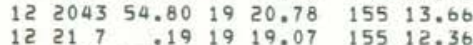

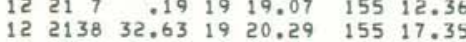
$\begin{array}{lllllll}12 & 2138 & 32.63 & 19 & 20.29 & 155 & 17.35 \\ 12 & 2339 & 12.12 & 19 & 21.37 & 155 & 13.77\end{array}$

$\begin{array}{lllllll}12 & 2356 & 20.82 & 19 & 21.01 & 155 & 6.91\end{array}$ $\begin{array}{lllllll}13 & 022 & 31.91 & 19 & 19.50 & 155 & 12.44 \\ 13 & 029 & 56.12 & 19 & 24.91 & 154 & 57.66\end{array}$

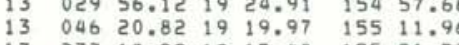

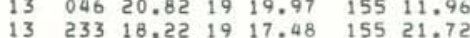

$\begin{array}{lllllll}13 & 256 & 43.88 & 19 & 19.92 & 155 & 14.24 \\ 13 & 344 & .09 & 19 & 20.51 & 155 & 8.55\end{array}$

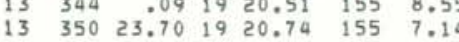

$\begin{array}{lllllllllll}7.08 & 2.5 & 2.8 & 25 & 0 & 95 & .11 & 7 & .7 & 1.2 & \text { UER }\end{array}$ $\begin{array}{llllllllll}9.57 & .4 & 2.8 & 27 & 0 & 64 & .14 & 9 & .9 & 1.2 \\ 0 & .2 & 0 E R\end{array}$

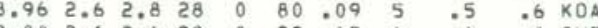

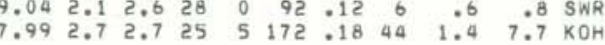

$\begin{array}{lllllllllll}4.49 & 4.2 & 4.2 & 32 & 0 & 61 & .11 & 13 & .7 & 1.8 & \text { GLN }\end{array}$

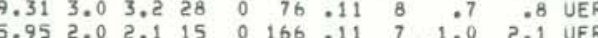

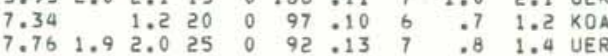
$\begin{array}{llllllllllll}1.51 & 1.7 & 1.6 & 17 & 0 & 124 & .13 & 3 & .6 & .3 & \mathrm{sPC}\end{array}$

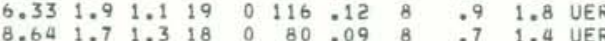

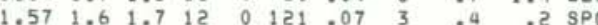
$\begin{array}{lllllllllll}9.30 & 2.7 & 2.9 & 29 & 0 & 55 & .13 & 7 & .7 & .8 & \text { UER }\end{array}$

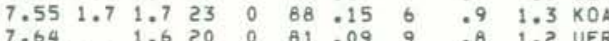

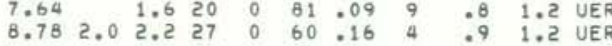
$6.162 .42 .929 \quad 0 \quad 83.14$ 6 2801.3 UER $\begin{array}{llllllllll}5.03 & .9 & 18 & 0 & 79 & .12 & 8 & .7 & 1.3 & \text { UER } \\ 8.13 & 1.2 & 19 & 0 & 89 & .09 & 9 & .8 & 1.1 & \text { UER }\end{array}$ $\begin{array}{llllllllllll}6.86 & 1.7 & 2.0 & 21 & 0 & 112 & .12 & 6 & .7 & 1.4 & \text { SWR }\end{array}$ $\begin{array}{lllllllll}0.86 & 1.0 & 16 & 0 & 98.09 & 5 & .6 & 1.3 & K O A\end{array}$

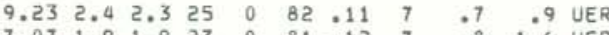

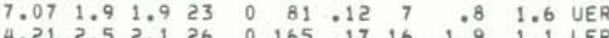
7.15 2.5 2.1 26 0165.17161 .90 1.1 LER

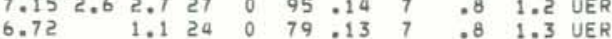
$\begin{array}{lllllllllll}6.65 & 1.6 & 1.4 & 22 & 0 & 80 & .15 & 6 & .9 & 2.0 & \text { UER }\end{array}$

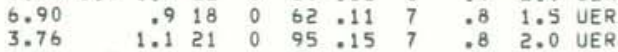
$\begin{array}{lllllllllll}3.76 & & 1.1 & 21 & 0 & 95 & .15 & 7 & .8 & 2.0 & \text { UER } \\ 6.82 & 1.5 & 1.7 & 22 & 0 & 62 & .12 & 5 & .7 & 1.1 & \text { KOA }\end{array}$

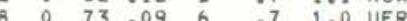

$\begin{array}{lllllllllll}8.96 & 3.2 & 3.2 & 29 & 0 & 89 & .11 & 7 & .7 & .6 & \text { UER }\end{array}$

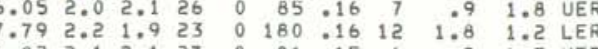

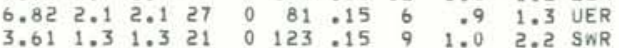
$\begin{array}{llllllllll}7.22 & 1.3 & 24 & 0 & 65 & .12 & 6 & .7 & 1.1 & \text { UER }\end{array}$

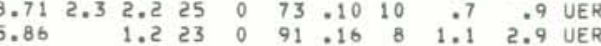

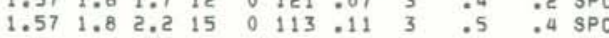

HVO EARTHQUAKE SUMMARY LIST YEAR MON ORIGIN TIME LAT LAT N SEC DEG MIN DEG MIN

$\begin{array}{llllllll}1976 & \text { JAN } 13 & 439 & 6.71 & 19 & 21.31 & 155 & 8.43\end{array}$ $\begin{array}{lllllll}13 & 459 & 26.05 & 19 & 17.79 & 155 & 22.49\end{array}$

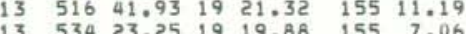

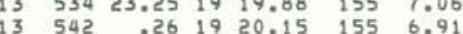

$\begin{array}{lllllllll}13 & 6 & 8 & 25.24 & 19 & 19.47 & 155 & 13.88\end{array}$ $\begin{array}{llllllll}13 & 617 & 54.90 & 19 & 18.97 & 155 & 13.63\end{array}$ $\begin{array}{llllllll}13 & 638 & 8.13 & 19 & 23.66 & 154 & 59.36\end{array}$

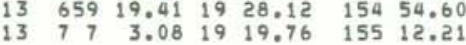

$\begin{array}{lllllll}13 & 722 & 38.73 & 19 & 17.71 & 155 & 21.51\end{array}$ $\begin{array}{lllllll}13 & 745 & 54.53 & 19 & 19.72 & 155 & 12.51\end{array}$ $13 \quad 92123.76 \quad 19$

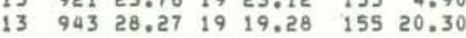
$\begin{array}{lllllll}13 & 955 & 34.85 & 19 & 20.40 & 155 & 17.73\end{array}$ $13.103018 .301920 .24 \quad 155 \quad 7.017$ $13 \quad 1046 \quad 45.80 \quad 19 \quad 19.98 \quad 155 \quad 17.77$ $13 \quad 1118 \quad 46.07 \quad 1921.34 \quad 154 \quad 59.75$ $\begin{array}{lllllll}13 & 1137 & 56.28 & 19 & 19.12 & 155 & 13.68\end{array}$ $\begin{array}{lllllll}13 & 1139 & 57.47 & 19 & 19.06 & 155 & 11.50 \\ 13 & 1143 & 46.43 & 19 & 20.34 & 155 & 16.70\end{array}$ $\begin{array}{lllllll}13 & 1213 & 35.54 & 19 & 21.17 & 155 & 8.13\end{array}$

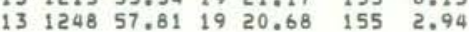
$\begin{array}{lllllll}13 & 1340 & 57.79 & 19 & 21.35 & 155 & 7.82\end{array}$

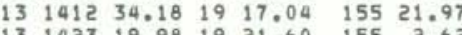
$13142319.901921 .60 \quad 1552.62$ $13 \quad 153134.65 \quad 1920.82 \quad 15511.08$

$\begin{array}{llllllll}13 & 16 & 1 & 35.23 & 19 & 22.29 & 155 & 6.54\end{array}$

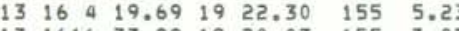
13161633.991920 .03 155 7.07 13162132.401920 .421558 .92

$\begin{array}{lllllll}13 & 1659 & 26.34 & 19 & 21.47 & 155 & 2.99\end{array}$ $\begin{array}{llllllll}13 & 17 & 8 & 36.59 & 19 & 24.83 & 154 & 59.12\end{array}$ $1317924.34 \quad 1920.68 \quad 155 \quad 9.91$

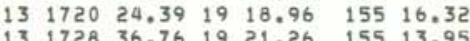

$\begin{array}{lllllll}13 & 1747 & 56.18 & 19 & 20.41 & 155 & 9.04\end{array}$

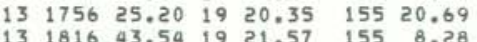

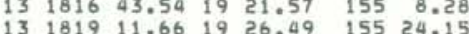
$\begin{array}{lllllll}13 & 1819 & 11.66 & 19 & 26.49 & 155 & 24.15 \\ 13 & 1824 & 9.61 & 19 & 17.58 & 155 & 20.61\end{array}$

$\begin{array}{lllllll}13 & 1843 & 54.02 & 19 & 22.38 & 155 & 5.04\end{array}$ $\begin{array}{lllllll}13 & 1850 & 27.59 & 19 & 19.71 & 155 & 18.04\end{array}$ $\begin{array}{lllllll}13 & 193 & 50.39 & 19 & 19,82 & 155 & 17.89\end{array}$
DEPTH AMP DUR GAP RMS MIN ERH ERZ $6.97 \quad 1.423 \quad 0 \quad 68 \quad 14 \quad 9 \quad 992.0$ UER

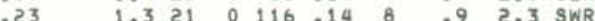
\begin{tabular}{llllllllll}
8.81 & 1.1 & 21 & 0 & 90 & .10 & 8 & .7 & 1.2 & 9 \\
\hline
\end{tabular}

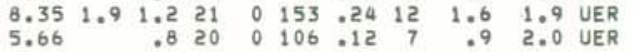

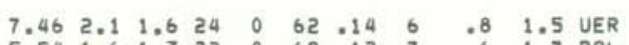

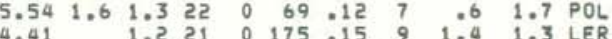

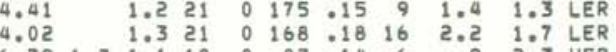

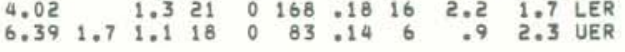

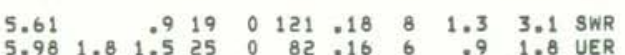

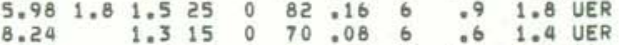

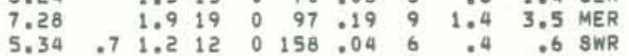
$\begin{array}{lllllllllll}7.29 & 1.8 & 2.4 & 22 & 0 & 65 & .10 & 5 & .6 & 1.1 & \mathrm{KOA}\end{array}$

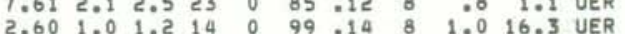
$\begin{array}{lllllllllll}7.34 & .6 & 1.1 & 8 & 0 & 130 & .06 & 6 & 1.0 & 2.6 & \text { KOA } \\ 3.76 & 1.4 & 1.8 & 14 & 2 & 206 & .18 & 12 & 1.8 & 2.5 & \text { LER }\end{array}$ $\begin{array}{llllllllll}6.94 & 1.6 & 18 & 0 & 82 & .12 & 7 & .9 & 2.0 & \text { UER }\end{array}$

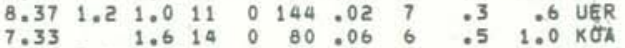

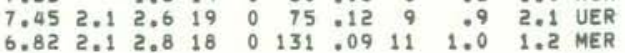
$\begin{array}{llllllllll}9.34 & 2.3 & 16 & 0 & 107 & .08 & 9 & .8 & 1.8 & \text { UER }\end{array}$ $\begin{array}{lllllllllll}3.41 & 1.1 & 1.7 & 20 & 2 & 126 & .20 & 8 & 1.2 & 3.7 & \text { SWR } \\ 5.94 & & 1.9 & 9 & 1 & 157 & .14 & 12 & 1.8 & 3.8 & \text { MER }\end{array}$

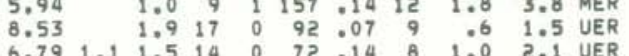
$\begin{array}{lllllllllll}6.79 & 1.1 & 1.5 & 14 & 0 & 72 & .14 & 8 & 1.0 & 2.1 & 1\end{array}$

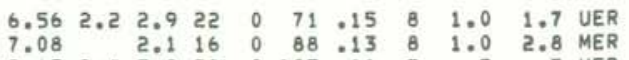

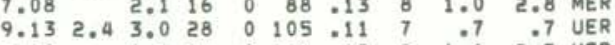

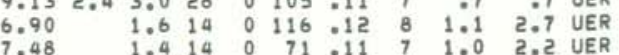
\begin{tabular}{llllllllll}
7.16 & 1.1 & 8 & 0 & 144 & .03 & 12 & .6 & 1.1 & MER \\
\hline & 1.5 & 8 & 0 & 163 & .32 & 11 & 9.1 & 3.4 & LER
\end{tabular}

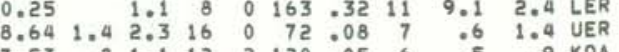
$\begin{array}{lllllllllll}7.53 & .8 & 1.1 & 12 & 2 & 120 & .05 & 6 & .5 & .9 & \text { KOA }\end{array}$

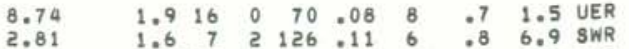

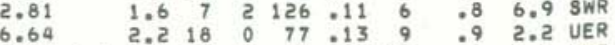
$\begin{array}{lllllllllll}7.91 & 1.2 & 1.1 & 13 & 0 & 94 & 0.09 & 10 & .9 & 2.0 & \text { UKF } \\ 5.52 & & 1.3 & 7 & 2 & 237 & .04 & 7 & 1.9 & .7 & \text { SWR }\end{array}$ $\begin{array}{llllllllll}6.42 & 1.1 & 1.4 & 19 & 0 & 92 & .15 & 8 & 1.1 & 3.0 \\ 5.5 & \text { MER }\end{array}$ $\begin{array}{lllllllllll}5.51 & .6 & 1.1 & 11 & 0 & 78 & .05 & 6 & .5 & .8 & \text { KOA } \\ 5.13 & & 1.3 & 15 & 0 & 77 & .09 & 6 & .6 & .9 & \text { KOA }\end{array}$ 
HVO EARTHQUAKE SUMMARY LIST

PAGE. 5

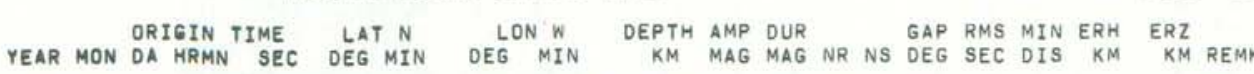

1976 JAN $13195 \quad 4.9919 \quad 19.63 \quad 15517.89$

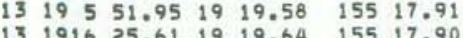
$13 \quad 1916 \quad 25.611919 .64 \quad 15517.90$

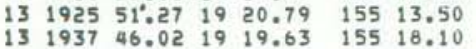

$\begin{array}{lllllll}13 & 1953 \quad 6.27 \quad 19 & 24.75 \quad 155 & 16.62\end{array}$ $131953 \quad 52.27 \quad 1924.75 \quad 15516.62$

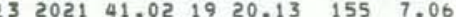
$\begin{array}{lllllll}13 & 2026 & 40.00 & 19 & 24.03 & 154 & 49.89 \\ 13 & 2027 & 43.11 & 19 & 26.29 & 155 & 23.23\end{array}$

$\begin{array}{lllllll}13 & 2046 & 33.27 & 19 & 23.57 & 155 & 25.32\end{array}$

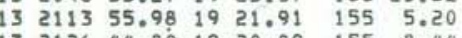
$132126 \quad 44.801920 .89155 \quad 8.44$

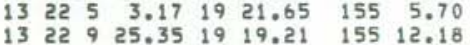

$\begin{array}{lllllll}13 & 2230 & 19.30 & 19 & 20.37 & 155 & 17.65\end{array}$ 130.02 $\begin{array}{lllllllll}13 & 23 & 2 & 57.90 & 19 & 19.85 & 155 & 16.29\end{array}$

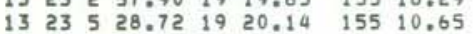

$\begin{array}{lllllll}13 & 2318 & 9.76 & 19 & 21.67 & 155 & 6.83\end{array}$ $\begin{array}{llrllll}13 & 2320 & 27.73 & 19 & 20.79 & 155 & 10.90 \\ 13 & 2346 & 8.94 & 19 & 19.07 & 155 & 22.54\end{array}$

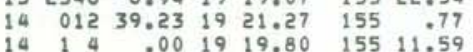

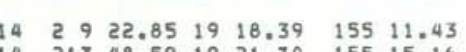
$14 \quad 213 \quad 48.591921 .70 \quad 155 \quad 15.16$

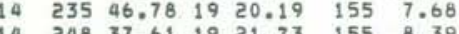
$14 \quad 34618.3919 \quad 20.80 \quad 155 \quad 10.69$

$\begin{array}{llllllll}14 & 4 & 8 & 56.19 & 19 & 22.03 & 155 & 1.09 \\ 14 & 414 & 58.41 & 19 & 19.83 & 155 & 12.56\end{array}$

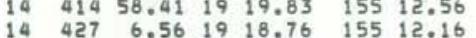

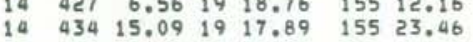

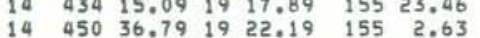

$\begin{array}{lllllll}14 & 452 & 14.09 & 19 & 27.05 & 155 & 23.54 \\ 14 & 544 & 41.32 & 19 & 21.81 & 155 & 15.43\end{array}$ $\begin{array}{lllllll}14 & 544 & 41.32 & 19 & 21.81 & 155 & 15.43 \\ 14 & 545 & 43.30 & 19 & 21.06 & 155 & 7.49\end{array}$

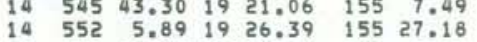

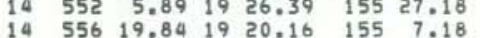

$\begin{array}{lllllll}14 & 557 & 15.92 & 19 & 19.99 & 155 & 12.71\end{array}$

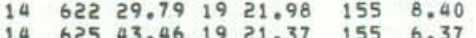

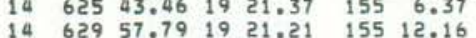
$14 \quad 6459.741921 .10 \quad 155 \quad 8.26$

$\begin{array}{lllllll}14 & 710 & 50.60 & 19 & 18.07 & 155 & 20.58\end{array}$ $\begin{array}{lllllll}14 & 713 & 54.91 & 19 & 18.55 & 155 & 16.67 \\ 14 & 722 & 26.97 & 19 & 19.90 & 155 & 12.47\end{array}$ $\begin{array}{lllllllllll}5.68 & .7 & 1.2 & 11 & 0 & 131 & .07 & 6 & .7 & 1.3 & K O A\end{array}$

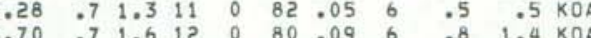
$\begin{array}{lllllllllll}.70 & .7 & 1.6 & 12 & 0 & 80 & .09 & 6 & .8 & 1.4 & \text { KOA }\end{array}$

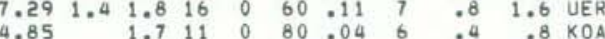

$\begin{array}{llllllllll}3.29 & 1.1 & 21 & 0 & 91 & .09 & 4 & .7 & 1.0 & \text { DEP }\end{array}$ $\begin{array}{lllllllllll}.70 & 1.8 & 2.1 & 18 & 0 & 76 & .09 & 9 & .6 & 1.8 & \text { UKF }\end{array}$ $\begin{array}{lllllllll}0.64 & 1.4 & 14 & 0 & 104 & 0.12 & 7 & .9 & 4.2\end{array}$

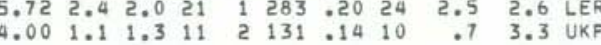

$\begin{array}{llllllllllll}7.29 & 1.3 & 1.5 & 14 & 0 & 133 & .09 & 10 & .9 & 2.0 & \text { UKF }\end{array}$

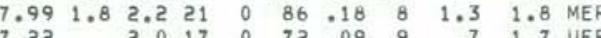

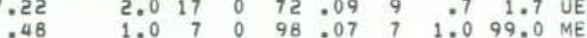

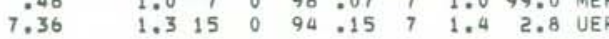
$\begin{array}{lllllllllll}7.39 & .8 & 1.1 & 16 & 0 & 67 & .13 & 5 & 1.0 & 1.9 & \text { KOA }\end{array}$

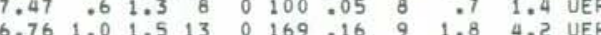
$\begin{array}{llllllllll}6.29 & 1.2 & 17 & 0 & 93 & 13 & 5 & 1.0 & 1.9 & \text { KOA }\end{array}$ $6.88 \quad 1.1 \quad 1.2$ 16 $00166: 11$ \& 1.2 1.9 UER $\begin{array}{lllllllll}7.04 & 1.3 & 18 & 0 & 78 & .16 & 8 & 1.2 & 2.2 \\ 7 & \text { UER }\end{array}$

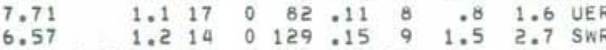
$\begin{array}{lllllllllll}5.86 & 1.3 & 1.3 & 19 & 0 & 218 & .23 & 13 & 3.3 & 2.4 & \text { LER } \\ 6.74 & & 2.3 & 23 & 0 & 87 & .12 & 6 & .7 & 1.6 & \end{array}$ $\begin{array}{lllllllllllll}5.57 & 1.1 & 1.0 & 15 & 0 & 127 & .14 & 7 & 1.2 & 3.6 & \text { POL }\end{array}$

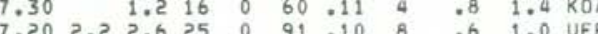

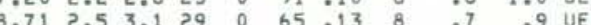

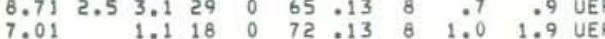
\begin{tabular}{lllllllll}
6.33 & 1.5 & 20 & 0 & 173 & .17 & 12 & 1.5 & 2.3 \\
\hline & MER
\end{tabular}

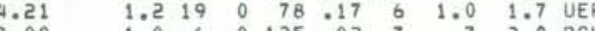

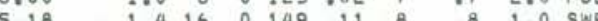

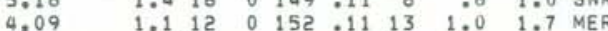

$9.62 \quad 1.617 \quad 0 \quad 93.1011 \quad 1.0 \quad 3.9$ UKF $\begin{array}{llllllll}9.9 & 0 & 1.5 & \text { KOA }\end{array}$ $8.451: 1 \quad 1: 1$ 11 $00118: 14$ 13 $1: 9$ 5.2 UKF $\begin{array}{lllllllll}4.55 & 2.1 & 21 & 0 & 73 & .17 & 6 & 1.0 & 1.5\end{array}$ $\begin{array}{llllllllll}0.1 .1 & 1.2 & 16 & 0 & 79 & .08 & 9 & .7 & 2.6 & \text { UER }\end{array}$

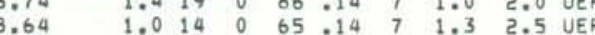

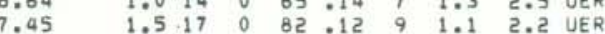

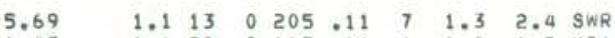

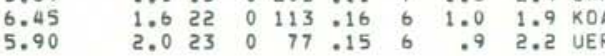

HVO EARTHDUAKE SUMMARY LIST

PAGE 6

ORIGIN TIME LATN LON N

$\begin{array}{lllll}\text { MN } & \text { DEPTH AMP DUR N } \\ \text { MIN } & \text { KM MAP RMS MIN ERH } & \text { ERZ } \\ \text { MAG NR NS DEG SEC OIS KM KM REMK }\end{array}$

$\begin{array}{llllllll}1976 & \text { JAN } 14 & 729 & 16.68 & 19 & 21.77 & 155 & 2.67\end{array}$

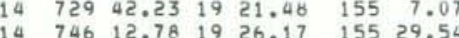
$\begin{array}{lllllll}14 & 746 & 12.78 & 19 & 26.17 & 155 & 29.5\end{array}$

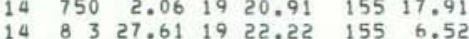
$\begin{array}{lllll}14 \quad 846 & 42.25 & 19 & 20.12 \quad 155 \quad 6.61\end{array}$

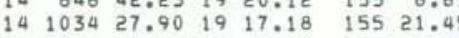
$1412951.841922 .15 \quad 155 \quad 6.54$ $14169 \begin{array}{llllll}14.54 & 19 & 17.93 & 155 & 21.26\end{array}$ $\begin{array}{lllllll}15 & 721 & 47.45 \quad 19 & 20.16 \quad 155 \quad 7.80\end{array}$ $19 \quad 155 \quad 8.6$ $15124145.311920 .55 \quad 15517.59$ $15 \quad 125926.091924 .65 \quad 155 \quad 17.60$

$\begin{array}{lllllll}15 & 1357 & 51.81 & 19 & 23.20 & 155 & 3.64\end{array}$ 15 22 $45.62 \quad 1922.64 \quad 155 \quad 6.49$

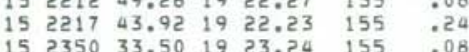
$\begin{array}{lllllll}16 & 1428 & 39.50 & 19 & 28.36 & 155 & 22.35\end{array}$

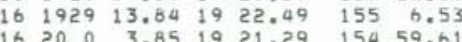

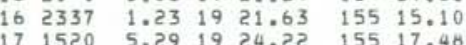
$\begin{array}{lllllll}17 & 1613 & 28.37 \quad 19 & 19.85 \quad 155 \quad 11.47\end{array}$

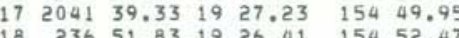
$18 \quad 23628.371926 .73$ 155 15.30

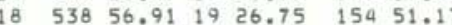
$\begin{array}{lllllll}18 & 817 & 52.38 & 19 & 20.21 & 155 & 6.80\end{array}$

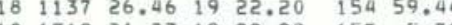

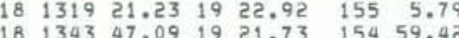
18 14 $5 \quad 10.92 \quad 1931.77 \quad 155 \quad 7.01$

$\begin{array}{lllllll}18 & 1413 & 37.89 & 19 & 21.65 & 155 & 7.62\end{array}$ $\begin{array}{lllllll}18 & 1520 & 31.05 & 19 & 20.43 & 155 & 3.91\end{array}$

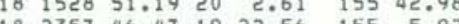
$190216.64 \quad 1923.13 \quad 155 \quad 7.26$

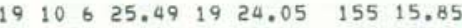
$19 \begin{array}{llllll}1853 & 37.22 & 19 & 18.97 & 155 & 22.23\end{array}$ 19235054.071924 .69 155 13.10

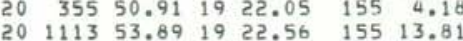

$\begin{array}{llllllll}20 & 15 & 0 & 32.72 & 19 & 21.82 & 155 & 3.57\end{array}$ $21 \quad 013 \quad 18.761929 .53 \quad 154 \quad 51.47$

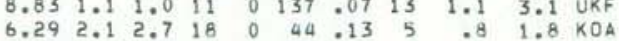
$\begin{array}{lllllllllll}7.68 & 2.1 & 2.6 & 28 & 0 & 111 & .12 & 7 & .6 & 1.0 & \text { UER }\end{array}$

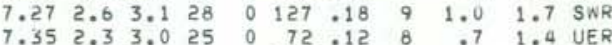

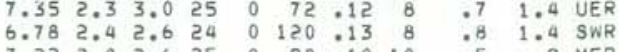

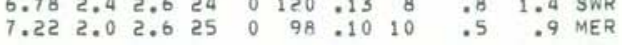

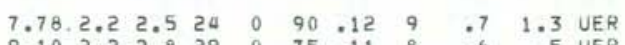
$\begin{array}{llllllllll}9.10 & 2.2 & 2.8 & 29 & 0 & 75 & .11 & 8 & .6 & .5 \\ \text { UER }\end{array}$

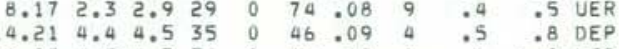

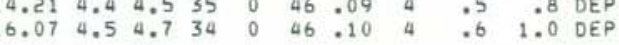
$\begin{array}{lllllllllll}7.69 & 2.5 & 2.9 & 29 & 0 & 109 & .13 & 11 & .8 & .9 & \text { MER }\end{array}$

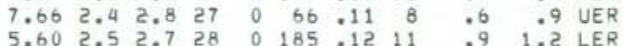

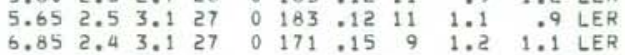
$\begin{array}{llllllllll}7.93 & 2.9 & 2.8 & 28 & 0 & 99 & .14 & 11 & .7 & 1.4 \\ \text { UKF }\end{array}$

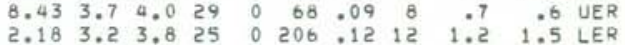

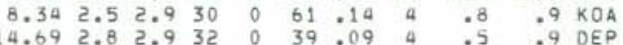

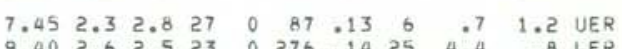

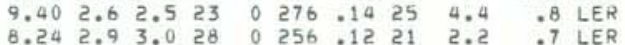

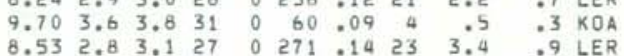

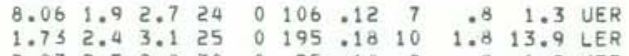

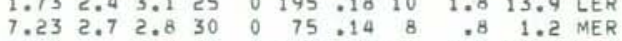

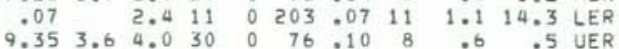
$\begin{array}{lllllllllll}7.03 & 3.6 & 3.7 & 31 & 0 & 73 & .09 & 8 & .5 & .7 & \text { UER }\end{array}$ 6.072 .63 .028 O 112.15 Q 1.111 .0 MER

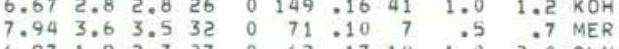

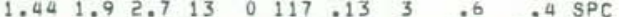

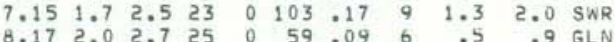

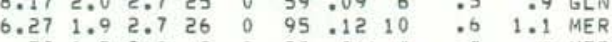

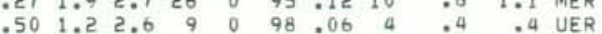

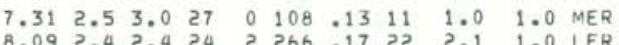

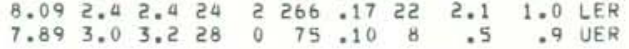
$\begin{array}{llllllllll}5.00 & 1.2 & 13 & 0 & 147 & .14 & 12 & 1.5 & 1.5 & \text { MER }\end{array}$

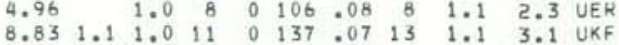

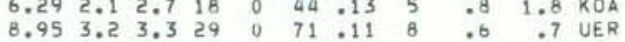


ORIGIN TIME LAT N LON W
OA HRMN SEC DEG MIN DEG MIN YEAR MON DA HRMN SEC DEG MIN DEG MIN 1976 JAN $21 \quad 114121.24 \quad 1922.02 \quad 155 \quad 7.21$ $\begin{array}{lllllll}21 & 2155 & 8.76 & 19 & 27.53 & 154 & 51.84 \\ 21 & 2225 & 24.22 & 19 & 23.91 & 155 & 3.29\end{array}$

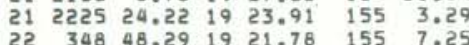

$\begin{array}{lllllll}22 & 412 & 3.95 & 19 & 20.09 & 155 & 8.49\end{array}$

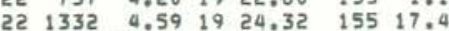
$\begin{array}{rrrrrrr}22 & 2016 & 24.70 & 19 & 19.87 & 155 & 8.64 \\ 23 & 145 & 47.84 & 19 & 18.54 & 155 & 23.63\end{array}$

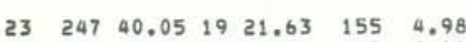
$\begin{array}{lllllll}23 & 247 & 40.05 & 19 & 21.63 & 155 & 4.98 \\ 23 & 5 & 52.40 & 19 & 19.57 & 155 & 9.99\end{array}$ $23 \quad 65912.14 \quad 1919.33 \quad 15511.82$ $\begin{array}{lllllll}23 & 739 & 20.97 & 19 & 20.69 & 155 & 17.62 \\ 23 & 752 & 20.09 & 19 & 19.46 & 155 & 13.97\end{array}$

$\begin{array}{lllllll}23 & 820 & 14.11 & 19 & 21.82 & 155 & 7.92\end{array}$ $23.01705 .0319 \quad 19.73 \quad 155 \quad 11.58$

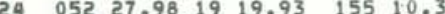

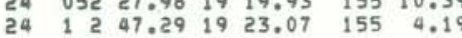

$\begin{array}{lllllll}24 & 251 & 7.39 & 19 & 20.31 & 155 & 7.31\end{array}$

c $24 \quad 134714.731919 .42 \quad 155 \quad 7.82$ $24 \quad 1455 \quad 43.66 \quad 1926.10 \quad 155 \quad 15.93$ $24164326.73 \quad 1920.42 \quad 155 \quad 16.65$ $\begin{array}{llllllll}25 & 016 & 42.31 & 19 & 19.50 & 155 & 12.69\end{array}$ $\begin{array}{lllllll}25 & 126 & 15.87 & 19 & 18.91 & 155 & 7.79\end{array}$ $25013 \quad 16.9818 \quad 54.26 \quad 15514.22$

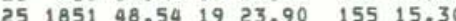

$\begin{array}{lllllll}25 & 1933 & 31.12 & 19 & 20.88 & 155 & 5.40\end{array}$

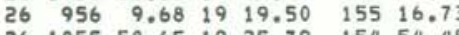
$26105558.651925 .79 \quad 15454.45$

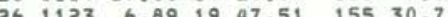

$\begin{array}{llllllll}26 & 13 & 0 & 4.04 & 19 & 27.33 & 154 & 53.15\end{array}$ $\begin{array}{lrrrrrr}26 & 1622 & 3.95 & 19 & 25.35 & 154 & 55.72 \\ 27 & 0 & 57.51 & 19 & 19.58 & 155 & 12.24\end{array}$ $27 \quad 131 \quad 18.791920 .12 \quad 155 \quad 7.60$

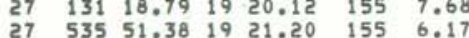

$\begin{array}{lllllll}27 & 610 & 27.17 & 19 & 21.01 & 155 & 13.36\end{array}$ $\begin{array}{rrrrrrr}27 & 1134 & 4.58 & 19 & 20.57 & 155 & 5.28 \\ 27 & 2047 & 58.40 & 19 & 32.02 & 155 & 13.39\end{array}$ $\begin{array}{lllllll}27 & 2047 & 58.40 & 19 & 32.02 & 155 & 13.39 \\ 27 & 2226 & 28.16 & 19 & 20.28 & 155 & 6.66\end{array}$ $\begin{array}{lrrrrrr}27 & 2226 & 28.16 & 19 & 20.28 & 155 & 6.64 \\ 28 & 330 & 37.81 & 18 & 53.24 & 155 & 14.88\end{array}$

$\begin{array}{lllllll}28 & 348 & 15.92 & 19 & 20.12 & 155 & 7.44\end{array}$

$\begin{array}{lllllll}28 & 641 & 27.79 & 19 & 19.02 & 155 & 20.04 \\ 28 & 956 & 41.57 & 19 & 22.97 & 155 & 6.34\end{array}$
OEPTH AMP OUR GAP RMS MIN ERH ERZ

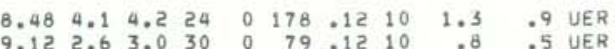
$\begin{array}{llllllllll}8.06 & 2.4 & 2.8 & 20 & 0 & 269 & .14 & 21 & 3.2 & .8 \\ 0.8 & \text { LER }\end{array}$

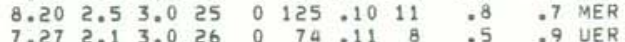

$\begin{array}{lllllllllll}8.50 & 2.8 & 3.4 & 28 & 0 & 77 & .09 & 9 & .5 & .7 & \text { UER }\end{array}$

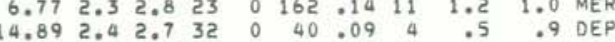
$8.312 .33 .029 \quad 0 \quad 76.09 \quad 9 \quad .5 \quad .7$ UER 4.573 .23 .0230105 .1300 .740 .2 SWR

$\begin{array}{lllllllllll}8.96 & 3.7 & 4.0 & 31 & 0 & 82 & .13 & 9 & .8 & .6 & \text { MER }\end{array}$

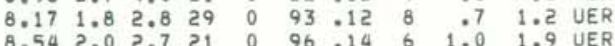

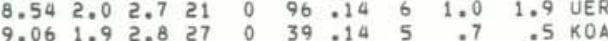

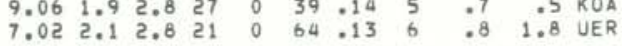
$\begin{array}{lllllllllll}8.91 & 3.2 & 3.5 & 26 & 0 & 79 & .10 & 8 & .6 & .7 & \text { UER }\end{array}$

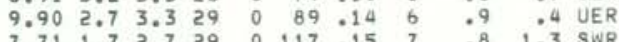

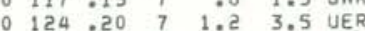
$8.472 .42 .9290099 .1010 \quad .6 \quad .7$ MER $\begin{array}{lllllllllll}7.69 & 1.9 & 2.7 & 29 & 0 & 96 & .13 & 8 & .8 & .7 & \text { UER }\end{array}$

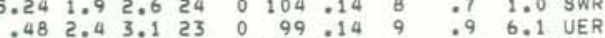

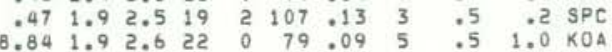
$\begin{array}{lllllllllll}6.23 & 2.0 & 2.5 & 25 & 0 & 81 & .16 & 7 & .9 & 1.8 & \text { UER }\end{array}$ .04 $9.862 .92 .822 \quad 00257.12$ 44 $4.5 \quad 97$ PPL

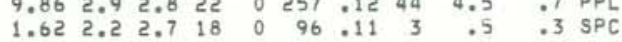
$\begin{array}{llllllllll}6.77 & 2.5 & 3.2 & 27 & 0 & 102 & .14 & 8 & .8 & 1.0 \\ 8 E R\end{array}$

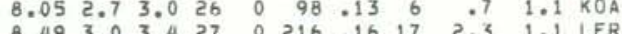

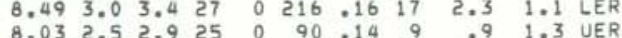
$31.842 .92 .6 \quad 31 \quad 1 \quad 95.14 \quad 21 \quad 1.123 .7 \quad$ KKU

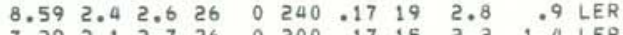

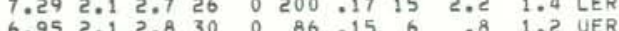
$1.311 .82 .520 \quad 1148.1310 \quad 991.7$ UER

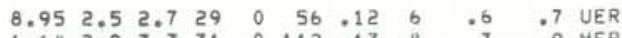

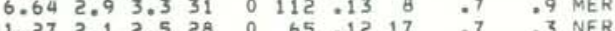

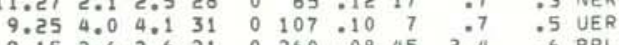
$\begin{array}{lllllllllll}8.47 & 1.9 & 2.8 & 24 & 0 & 97 & .11 & 8 & .6 & .9 & \text { UER }\end{array}$ $\begin{array}{lllllllllll}9.30 & 2.2 & 2.8 & 25 & 0 & 90 & .14 & 6 & .8 & 1.1 & \text { SNR } \\ 6.24 & 2.5 & 2.9 & 29 & 0 & 69 & .16 & 8 & .8 & 1.4 & \text { UER }\end{array}$
ORIGIN TIME LAT N LON N
DA HRMN SEC DEG MIN DEG MIN VEAR MON DA HRMN TINEC DEG MIN COEG MIN $\begin{array}{lllllll}28 & 1845 & 25.87 & 19 & 19.74 & 155 & 11.33\end{array}$

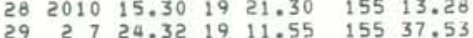
$\begin{array}{llllllll}29 & 2 & 7 & 24.32 & 19 & 11.55 & 155 & 37.53 \\ 29 & 5 & 0 & 56.17 & 19 & 20.61 & 155 & 6.46\end{array}$

29 $1019 \quad 56.47 \quad 19 \quad 22.74 \quad 154 \quad 59.64$

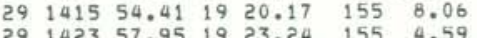

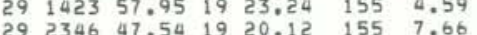

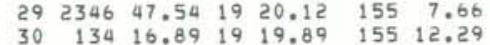

$\begin{array}{lllllll}30 & 2027 & 14.32 & 19 & 19.31 & 155 & 13.25\end{array}$ $\begin{array}{lllllll}31 & 122 & .20 & 19 & 26.61 & 155 & 22.54\end{array}$

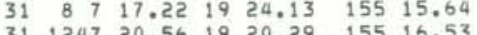
$\begin{array}{lllllll}31 & 1247 & 20.56 & 19 & 20.29 & 155 & 16.53 \\ 31 & 1345 & 48.81 & 19 & 25.27 & 154 & 54.51\end{array}$

$\begin{array}{lllllll}31 & 1632 & 9.40 & 19 & 20.77 & 155 & 7.37\end{array}$ $\begin{array}{lllllll}31 & 1759 & 11.76 & 19 & 27.86 & 154 & 53.62\end{array}$

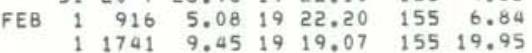
$\begin{array}{lllllll}2 & 332 & 18.25 & 19 & 20.93 & 155 & 11.12\end{array}$

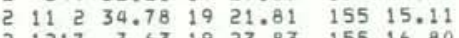

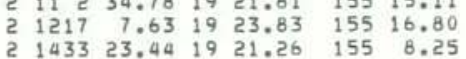

$\begin{array}{lllllll}3 & 739 & 57.67 & 19 & 20.75 & 155 & 4.02\end{array}$

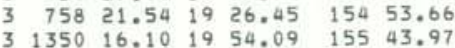
$\begin{array}{llllllll}3 & 1454 & 23.30 & 19 & 20.40 & 155 & 13.68\end{array}$

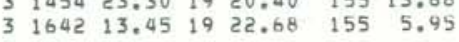

$\begin{array}{lllllll}3 & 1737 & 6.19 & 19 & 18.37 & 155 & 20.48\end{array}$ $\begin{array}{lllllll}3 & 2045 & 21.72 & 19 & 20.30 & 155 & 11.73 \\ 3 & 2050 & 58.46 & 19 & 21.67 & 155 & 15.73\end{array}$

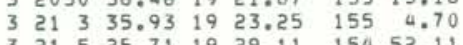

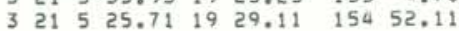

$\begin{array}{llllllll}4 & 5 & 37.85 & 19 & 23.38 & 155 & .67 \\ 4 & 848 & 41.68 & 19 & 20.29 & 155 & 4.57\end{array}$

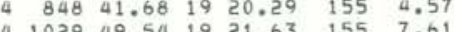
$4102909.541921 .63 \quad 155 \quad 7.61$ $4 \quad 1533 \quad 39.32 \quad 1939.55 \quad 155 \quad 6.40$ $5 \quad 1721.2019 \quad 19.11 \quad 15511.50$

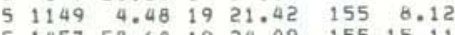
5 1457 58.601924 .09 155 15.11 $6 \quad 1 \quad 6.19 \quad 19 \quad 19.65 \quad 15520.07$

$\begin{array}{lllllll}6 & 139 & .29 & 19 & 21.59 & 155 & 8.17\end{array}$ $\begin{array}{rrrrrrr}6 & 240 & 7.96 & 19 & 20.82 & 155 & 8.69 \\ 6 & 844 & 15.49 & 19 & 28.00 & 154 & 51.45\end{array}$
GAP RMS MIN ERH ERZ MAG MAG NR NS DEG SEC DIS KM KM REMK $\begin{array}{llllllllllll}9.29 & 2.8 & 2.7 & 29 & 0 & 99 & 112 & 7 & .8 & .7 & \text { UER } \\ 9.79 & 2.5 & 3.1 & 26 & 0 & 90 & 11 & 6 & .6 & 1.0 & \text { UER }\end{array}$

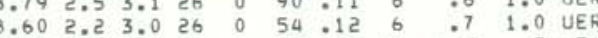
9.853 .033 .4425 0 97.22 .21 1.5 .7 HEA \begin{tabular}{llllllllll}
8.66 & 4.7 & 4.9 & 33 & 0 & 184 & .14 & 10 & 1.2 & .9 \\
\hline
\end{tabular}

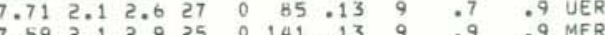

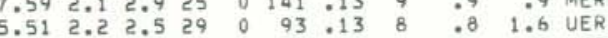

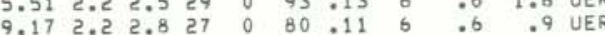

$\begin{array}{lllllllllll}10.09 & 2.8 & 3.1 & 26 & 0 & 74 & .13 & 7 & .8 & .4 & \text { UER }\end{array}$

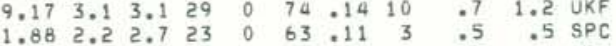
$9.332 .83 .332 \quad 0 \quad 82.1160 .5$. 6 KO

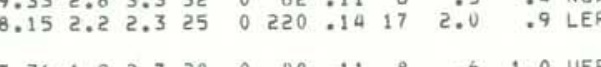
$\begin{array}{lllllllllll}7.36 & 1.9 & 2.7 & 28 & 0 & 88 & .11 & 8 & .6 & 1.0 & \text { UER }\end{array}$

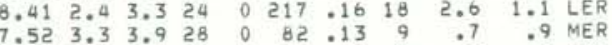

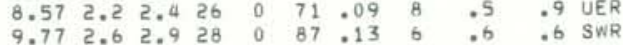
$\begin{array}{lllllllllll}8.87 & 2.2 & 2.6 & 28 & 0 & 70 & .14 & 8 & .8 & 1.1 & \text { UER }\end{array}$ \begin{tabular}{llllllllll}
7.87 & 1.8 & 1.9 & 16 & 4 & 185 & .19 & 16 & 1.8 & 1.1 \\
\hline & LER
\end{tabular} $8.311 .9 \begin{array}{lllllllll}2.5 & 26 & 0 & 59 & .12 & 4 & .6 & .9 & \text { KOA }\end{array}$ \begin{tabular}{llllllllll}
1.51 & 1.7 & 2.3 & 15 & 0 & 80 & .10 & 3 & .5 & .3 \\
9.31 & 2.1 & 2.5 & 26 & 0 & 71 & .07 & 9 & .4 & .5 \\
\hline
\end{tabular}

$\begin{array}{lllllllllll}5.57 & 2.0 & 2.5 & 25 & 0 & 114 & .15 & 10 & 1.0 & 1.7 & \text { MER }\end{array}$

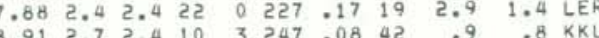

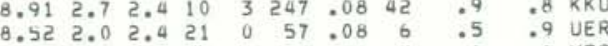
$8.743 .43 .730 \quad 0 \quad 72.11 \quad 7 \quad .6 \quad .6 \mathrm{MER}$ $\begin{array}{llllllllllll}9.56 & 2.5 & 3.1 & 28 & 0 & 115 & .15 & 6 & .9 & .7 & \text { SWR }\end{array}$ 9.783 .53 .8290001 .08 4 0.4 . 3 KOA

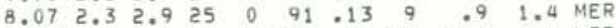

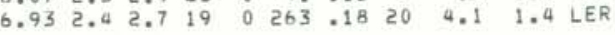
$\begin{array}{llllllllll}6.75 & 2.4 & 3.0 & 24 & 0 & 160 & .08 & 9 & .7 & .5 \\ 7 & \text { LER }\end{array}$ 7.0362 .83 .327 0 73 .11 8 O 9 UER

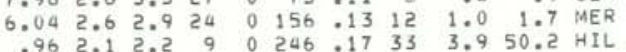
$\begin{array}{llllllllllll}9.01 & 1.8 & 2.2 & 18 & 0 & 104 & .09 & 7 & .7 & 1.2 & \text { UER }\end{array}$ $\begin{array}{lllllllllll}8.14 & 3.1 & 3.6 & 28 & 0 & 71 & .12 & 9 & .7 & .9 & \text { UER } \\ 2.00 & 1.0 & 3.01 & 12 & 0 & 117 & .22 & 3 & 1.8 & 90 & \text { SPC }\end{array}$ 9.273 .23 .628 0 $96.08 \quad 7 \quad .0 \quad .4$ UER

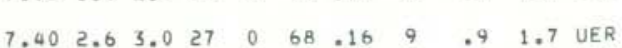

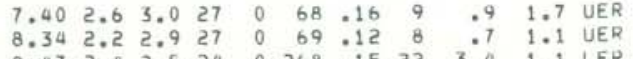

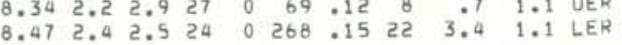


ORIGIN TIME LATN LON W DEPTH AMP OUR G GAP RMS MIN ERH ERZ

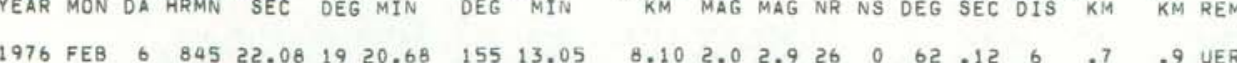
6
1848 $31.451925 .60 \quad 15525.49$

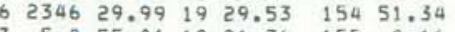

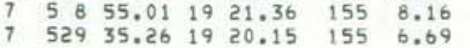

$\begin{array}{lllllll}7 & 742 & 42.77 & 19 & 19.10 & 155 & 22.05\end{array}$

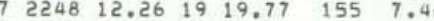
$\begin{array}{lllllll}7 & 2346 & 41.99 & 19 & 20.46 & 155 & 6.78\end{array}$

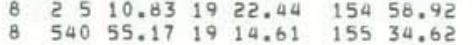

$\begin{array}{llllllll}8 & 9 & 3 & 49.18 & 19 & 19.43 & 155 & 19.10\end{array}$

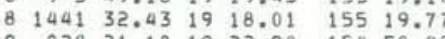

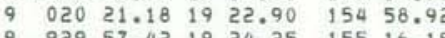

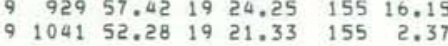

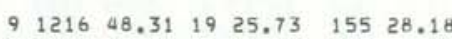
$\begin{array}{llllll}1457 & 17.20 & 19 & 21.86 & 155 & 3.61\end{array}$

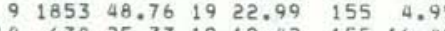
$\begin{array}{lllllll}10 & 638 & 25.33 & 19 & 19.42 & 155 & 16.49 \\ 10 & 839 & 39.73 & 19 & 20.33 & 155 & 13.27\end{array}$

$\begin{array}{lllllll}10 & 1639 & 53.69 & 19 & 20.06 & 155 & 12.28\end{array}$

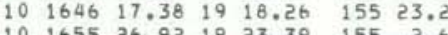
$10165526.92 \quad 1923.39 \quad 155 \quad 2.64$ $10 \quad 1820 \quad 8.5519 \quad 20.33 \quad 155 \quad 6.87$

$\begin{array}{lllllll}10 & 1956 & 7.78 & 19 & 21.31 & 155 & 7.39\end{array}$

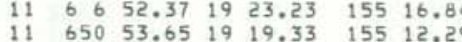
$\begin{array}{lllllll}11 & 1457 & 22.73 & 19 & 28.20 & 154 & 49.40\end{array}$

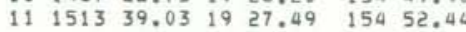

$\begin{array}{lllllll}11 & 1740 & 5.06 & 19 & 21.75 & 155 & 6.24\end{array}$

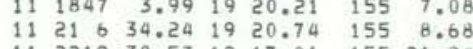

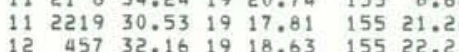
$\begin{array}{lllllll}12 & 618 & 26.21 & 19 & 21.67 & 155 & 3.5\end{array}$ $\begin{array}{lllllll}12 & 1531 & 19.56 & 19 & 20.84 & 155 & 6.51\end{array}$ $12192653.561922 .06 \quad 15517.96$ $12 \quad 211443.3519 \quad 18.68 \quad 155233.01$

$\begin{array}{lllllll}13 & 049 & 51.86 & 19 & 31.33 & 155 & 57.70\end{array}$

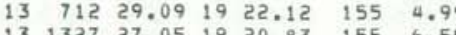

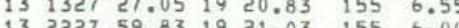
$14049 \quad 47.221923 .07 \quad 155 \quad 3.02$

$\begin{array}{lllllll}14 & 1356 & 27.52 & 19 & 27.03 & 154 & 54.87\end{array}$

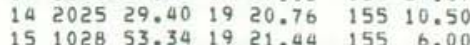

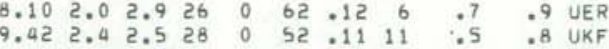

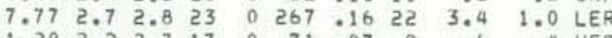

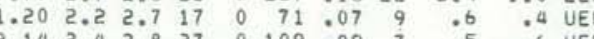

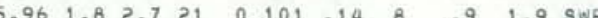

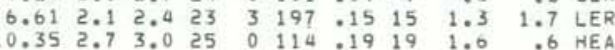

$\begin{array}{lllllllllll}9.06 & 1.7 & 3.2 & 27 & 0 & 57 & .13 & 7 & .7 & .8 & \text { SWR }\end{array}$

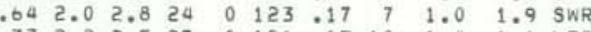
$\begin{array}{lllllllllll}6.33 & 2.2 & 2.5 & 27 & 0 & 191 & .15 & 10 & 1.4 & 1.1 & \text { LER }\end{array}$

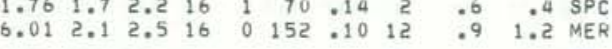

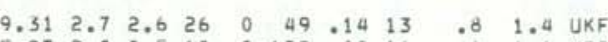
$\begin{array}{llllllllll}.97 & 2.0 & 2.5 & 18 & 0 & 122 & .09 & 11 & .6 & 1.1 \\ 1.11 & \text { MER }\end{array}$

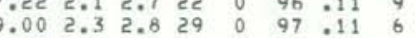

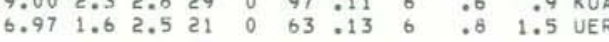
$\begin{array}{lllllllllll}7.01 & 1.8 & 2.6 & 19 & 0 & 77 & .10 & 6 & .7 & 1.4 & \text { UER }\end{array}$

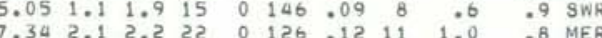

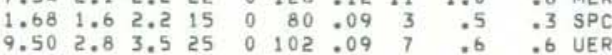
$\begin{array}{lllllllllll}7.85 & 2.5 & 2.8 & 24 & 0 & 79 & .10 & 8 & .6 & 1.0 & \text { UER }\end{array}$ $\begin{array}{lllllllllll}1.93 & 1.8 & 2.8 & 20 & 0 & 57 & .13 & 2 & .6 & .3 & \text { SPC } \\ 7.23 & 1.7 & 2.5 & 23 & 0 & 90 & .11 & 7 & .7 & .5 & .5\end{array}$ $8.012 .52 .419 \quad 0277.1525 \quad 6.44 \quad 1.2$ LER $\begin{array}{llllllllll}7.43 & 2.1 & 2.6 & 26 & 0 & 80 & .13 & 9 & .7 & 1.2\end{array}$ UER

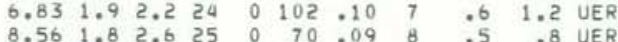

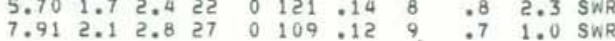

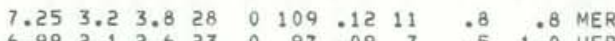

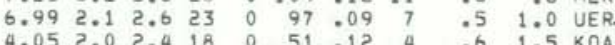

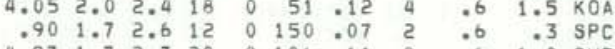

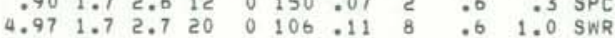

$\begin{array}{rrrrrrrrrrr}9.68 & 3.6 & 3.1 & 27 & 0 & 234 & .15 & 22 & 2.1 & .4 & \text { KON } \\ 7.14 & 2.9 & 3.1 & 28 & 0 & 80 & .10 & 9 & .5 & 1.0 & \text { MER }\end{array}$

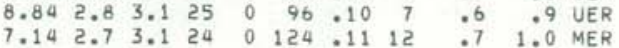

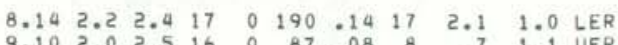
$\begin{array}{rrrrrrrrrrr}9.10 & 2.0 & 2.5 & 16 & 0 & 87 & .08 & 8 & .7 & 1.1 & \text { UER } \\ 8.39 & 2.3 & 3.1 & 16 & 0 & 111 & .09 & 8 & .7 & 1.1 & \text { UER }\end{array}$

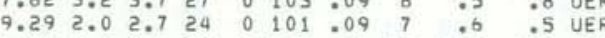

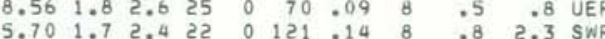

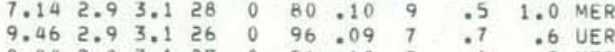

ORIGIN TIME LATN LON W YEAR MON DA MRMN SEC DEG MIN OEG MIN

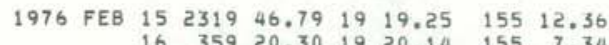

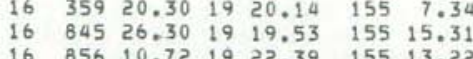
$\begin{array}{rrrrrrr}16 & 856 & 10.72 & 19 & 22.39 & 155 & 13.22 \\ 16 & 1043 & 59.96 & 19 & 20.66 & 155 & 6.47\end{array}$

$\begin{array}{lllllll}16 & 1045 & 24.32 & 19 & 21.15 & 155 & 2.59\end{array}$ $16 \quad 1136 \quad 11.46 \quad 1921.83 \quad 15513.01$ $\begin{array}{lllllll}16 & 1219 & 34.16 & 19 & 19.49 & 155 & 12.58\end{array}$ $\begin{array}{lllllll}16 & 1320 & 37.99 & 19 & 19.90 & 155 & 7.24 \\ 16 & 1411 & 48.31 & 19 & 20.56 & 155 & 6.58\end{array}$ $\begin{array}{lllllll}16 \quad 1418 & 26.81 & 19 & 19.76 \quad 155 \quad 16.74\end{array}$ $16 \quad 1419 \quad 2.531919 .79 \quad 155 \quad 15.16$ $\begin{array}{lllllll}16 & 1517 & 21.38 & 19 & 19.74 & 155 & 15.31\end{array}$

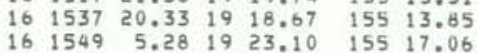

$\begin{array}{llllllll}16 & 17 & 0 & 23.77 & 19 & 19.24 & 155 & 20.99\end{array}$ $16 \quad 175 \quad 7.001918 .02 \quad 15515.15$

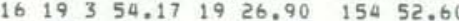

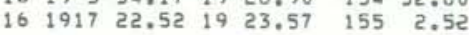
$\begin{array}{lllllll}16 & 1918 & 32.94 & 19 & 22.04 & 155 & 1.85\end{array}$ $\begin{array}{lllllll}16 & 1920 & 1.75 & 19 & 19.12 & 155 & 13.47\end{array}$ $162056 \quad 7.401919 .07 \quad 155 \quad 16.38$

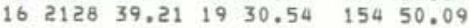
$\begin{array}{lllllll}16 & 2150 & 33.41 & 19 & 22.91 & 155 & .38\end{array}$

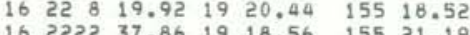
$16 \quad 234146.16 \quad 1919.16 \quad 155 \quad 15.44$

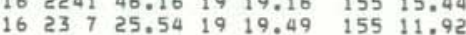

$\begin{array}{lllllll}17 & 017 & 15.37 & 19 & 28.07 & 154 & 51.61\end{array}$ $\begin{array}{lllllll}17 & 026 & 5.94 & 19 & 21.16 & 155 & 4.01\end{array}$

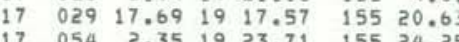
$17 \quad 140 \quad 55.4519 \quad 17.71 \quad 155 \quad 23.50$

$\begin{array}{lllllll}17 & 237 & 58.15 & 19 & 20.30 & 155 & 10.38\end{array}$ $\begin{array}{lllllll}17 & 241 & 6.19 & 19 & 20.62 & 155 & 8.52\end{array}$

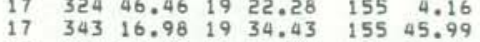

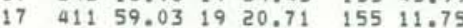

$\begin{array}{lllllll}17 & 532 & 2.18 & 19 & 23.91 & 155 & 16.00 \\ 17 & 553 & 7.59 & 19 & 23.61 & 154 & 59.97\end{array}$ 17 6 4 21.921919 .6415512 .30 $176062.831919 .86 \quad 155 \quad 7.69$

$77 \begin{array}{llllll}7 & 6.97 \quad 19 & 20.36 & 155 & 9.35\end{array}$

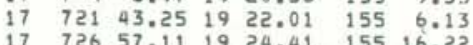

DEPTH AMP DUR GAP RMS MIN ERH ERZ 7.372 .43 .025 o $91.12 \quad 7$

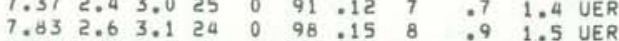

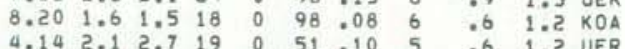

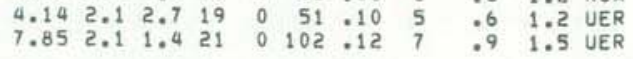

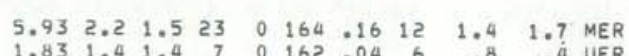

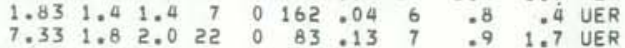
\begin{tabular}{rrrrrrrrrr}
11.54 & 1.4 & 16 & 0 & 105 & .11 & 8 & 1.2 & .5 & UER \\
8.42 & 2.5 & 2.7 & 24 & 0 & 101 & .11 & 7 & .7 & .8 \\
\hline
\end{tabular} $\begin{array}{llllllllll}7.12 & 1.1 & 17 & 0 & 94 & .12 & 5 & .9 & 1.7 & \mathrm{KOA}\end{array}$

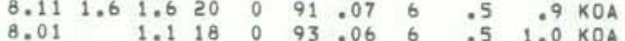

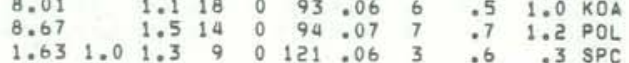
$\begin{array}{llllllllll}5.87 & 1.6 & 19 & 0 & 123 & .13 & 6 & .9 & 2.0 & \text { SWR }\end{array}$

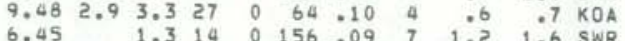

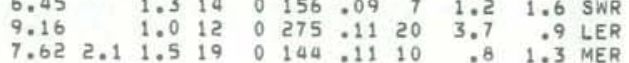
$\begin{array}{llllllllll}5.18 & 2.1 & 1.6 & 19 & 0 & 177 & .17 & 12 & 2.1 & 1.6 \\ 9 E R\end{array}$

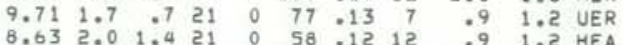

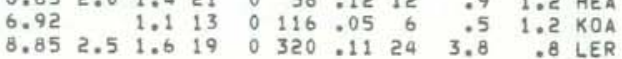
$\begin{array}{lllllllll}4.01 & 1.4 & 18 & 0 & 199 & .26 & 10 & 2.8 & 2.1 \\ 7.43 & 1.40 & \text { LER }\end{array}$

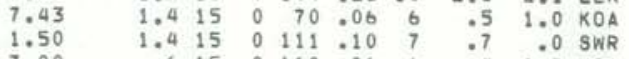

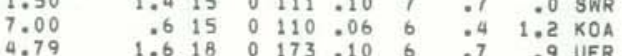
\begin{tabular}{lllllllll}
7.74 & 1.2 & 18 & 0 & 267 & .15 & 22 & 3.9 & 1.1 \\
\hline
\end{tabular}

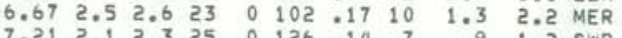

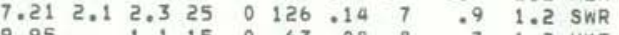
$\begin{array}{llllllllll}9.95 & 1.1 & 15 & 0 & 63 & 0.08 & 8 & .7 & 1.2 & \text { UKF } \\ 7.88 & 1.2 & 15 & 0 & 157 & .13 & 8 & 1.3 & 1.5 & \text { SWR }\end{array}$ $\begin{array}{llllllllllll}8.52 & 1.1 & 17 & 0 & 88 & .09 & 7 & .7 & 1.6 & \text { UER }\end{array}$

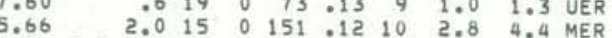

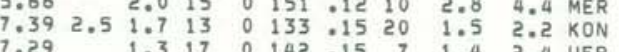
$\begin{array}{lllllllllllll}3.00 & 1.9 & 2.3 & 18 & 2 & 105 & 0.15 & 3 & -7 & 2.0 & \text { sPC }\end{array}$

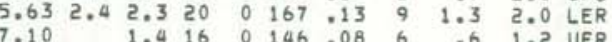

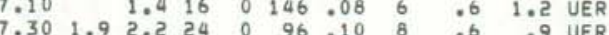

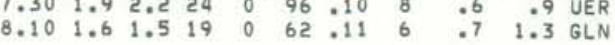
$\begin{array}{llllllllll}7.62 & 1.4 & 20 & 0 & 73 & .10 & 8 & .7 & 1.4 & \text { UER }\end{array}$ $\begin{array}{lllllllllll}6.91 & 1.6 & 23 & 0 & 76 & 1.14 & 7 & .9 & 1.6 & \text { UER } \\ 1.83 & 9 & 1.1 & 8 & 0 & 138 & .04 & 3 & 94 & .2 & \text { SPC }\end{array}$ 
HVO EARTHQUAKE SUMMARY LIST

PAGE 11 $\begin{array}{lllllll}\text { ORIGIN TIME } & \text { LAT N } & \text { LON W } & \text { DEPTH AMP DUR } & \text { GAP RMS MIN ERH ERZ } \\ \text { OA HRMN SEC DEG MIN DEG MIN } & \text { KM MAG MAG NR NS DEG SEC DIS KM KM REMK }\end{array}$ $\begin{array}{lllllll}1976 \quad F E B & 17 \quad 752 & 16.59 & 19 & 24.14 & 155 & 15.93\end{array}$

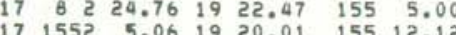
$\begin{array}{lllllll}17 & 17 & 21.74 & 19 & 17.65 & 155 & 21.11 \\ 17 & 1931 & 59.20 & 19 & 13.23 & 155 & 34.04\end{array}$ $\begin{array}{lllllll}18 & 425 & 36.76 \quad 19 & 20.12 \quad 155 \quad 7.47\end{array}$ $\begin{array}{lllllll}18 & 425 & 36.76 & 19 & 20.12 & 155 & 7.47 \\ 18 & 514 & 37.65 & 19 & 20.25 & 155 & 6.71\end{array}$ $18 \quad 1.050 \quad 47.59 \quad 19 \quad 27.07 \quad 15452.51$

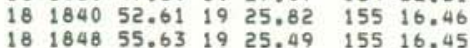
$\begin{array}{lllllll}18 & 2020 & 23.77 & 19 & 17.41 & 155 & 21.18\end{array}$

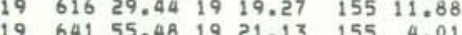
$19938 \quad 41.33 \quad 1928.56 \quad 154 \quad 49.92$ $192239 \quad 13.35 \quad 1926.46 \quad 155 \quad 25.11$ $\begin{array}{lllllll}19 & 2321 & 45.75 & 19 & 21.56 & 155 & 1.97\end{array}$ $\begin{array}{lllllll}20 & 533 & 1.70 & 19 & 22.31 & 155 & 6.34\end{array}$ $\begin{array}{lllllll}20 & 1332 & 45.36 & 19 & 18.03 & 155 & 22.05\end{array}$ $201951 \quad 14.912024 .95 \quad 156 \quad 1.29$

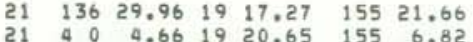

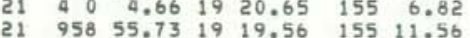

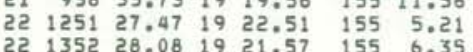

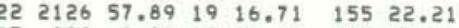
$\begin{array}{rrrrrrr}23 & 020 & 8.08 & 19 & 21.12 & 155 & 13.18 \\ 23 & 426 & 47.70 & 19 & 13.87 & 155 & 22.61\end{array}$ $\begin{array}{lllllll}23 & 426 & 47.70 & 19 & 13.87 & 155 & 22.61 \\ 23 & 72 & 28.80 & 19 & 22.18 & 155 & 12.98\end{array}$

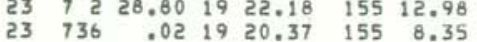

$\begin{array}{lllllll}23 & 855 & 49.91 & 19 & 22.98 & 155 & 4.92 \\ 23 & 945 & 23.48 & 19 & 21.61 & 155 & 15.24\end{array}$ $\begin{array}{lllllll}23 & 945 & 23.48 & 19 & 21.61 & 155 & 15.24 \\ 23 & 950 & 13.85 & 19 & 24.83 & 155 & 24.75\end{array}$ $\begin{array}{rrrrrrr}23 & 950 & 13.85 & 19 & 24.83 & 155 & 24.75 \\ 23 & 1150 & 2.34 & 19 & 24.75 & 155 & 24.63\end{array}$ 331828 . 450 $\begin{array}{lllllll}24 & 248 & 43.18 & 19 & 21.56 & 155 & 7.22\end{array}$

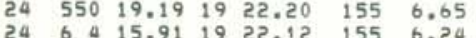
$\begin{array}{rrrrrrrr}24 & 6 & 4 & 15.91 & 19 & 22.12 & 155 & 6.24 \\ 24 & 9 & 0 & .18 & 19 & 27.51 & 155 & 28.33\end{array}$ $\begin{array}{lllllll}24 & 1442 & 32.63 & 19 & 23.27 & 155 & 4.81\end{array}$ $\begin{array}{llllll}24 & 1623 & 9.77 & 19 & 22.13 & 155 \quad 6.81\end{array}$

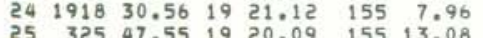

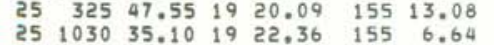

$\begin{array}{llllll}25 & 1035 & 50.63 \quad 19 & 19.85 & 155 & 7.92\end{array}$

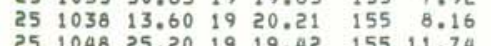

$\begin{array}{lllllllllllll}1.67 & 1.7 & 2.2 & 14 & 0 & 113 & .13 & 3 & .7 & .6 & \text { SPC }\end{array}$ $\begin{array}{llllllllll}7.50 & 1.5 & 18 & 0 & 93 & .12 & 9 & .9 & 1.8 & \text { MER } \\ 6.97 & 2.0 & 2.6 & 22 & 0 & 79 & 12 & 6 & .8 & 1.5\end{array}$

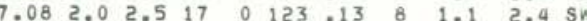
$9.392 .72 .922 \quad 1122.1820 \quad 1.4 \quad 1.6 \mathrm{HEA}$ $\begin{array}{lllllllllll}8.12 & 2.5 & 3.0 & 25 & 0 & 96 & .11 & 8 & .7 & .8 & \text { UER }\end{array}$ $8.672 .42 .012101259 .1521 \quad .6 \quad 1.2$ UER

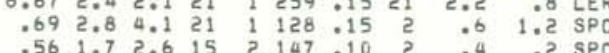
$\begin{array}{lllllllllll}7.48 & 1.9 & 2.6 & 21 & 0 & 126 & .11 & 8 & .8 & 1.3 & \text { SWR }\end{array}$

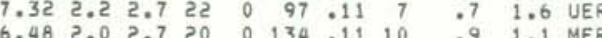

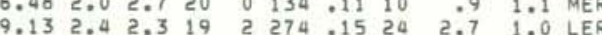

$\begin{array}{lllllllllll}8.19 & 2.5 & 2.7 & 25 & 0 & 161 & .11 & 13 & 1.0 & .7 & \text { MER }\end{array}$

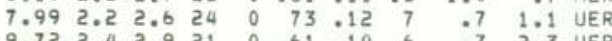

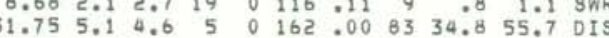

$\begin{array}{llllllllll}7.51 & 2.4 & 2.9 & 27 & 0 & 125 & .18 & 9 & 1.2 & 1.4\end{array}$ SWR

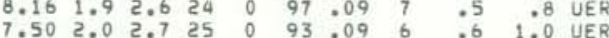
$9.03 \quad 2.53 .021 \quad 0 \quad 90.08 \quad 8 \quad .6 \quad 1.1$ MER $\begin{array}{lllllllllll}3.13 & 1.8 & 2.6 & 18 & 0 & 129 & .15 & 8 & 1.0 & 3.8 & \text { SWR }\end{array}$

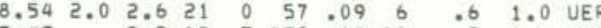

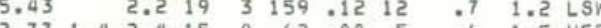

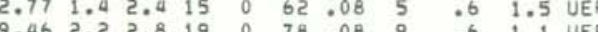

$\begin{array}{lllllllllllll}7.48 & 2.6 & 3.0 & 22 & 0 & 87 & .12 & 9 & .7 & 1.2 & \text { MER }\end{array}$

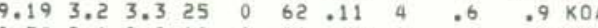
\begin{tabular}{llllllllll}
0.34 & 2.6 & 2.9 & 18 & 0 & 86 & 0.08 & 9 & .6 & .5 \\
\hline
\end{tabular}

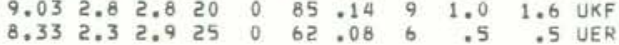

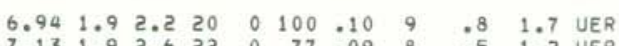
$\begin{array}{lllllllllll}7.13 & 1.9 & 2.6 & 22 & 0 & 77 & .09 & 8 & .5 & 1.2 & \text { UER }\end{array}$ $8.594 .2 \begin{array}{lllllllll}8.2 & 29 & 0 & 71 & 11 & 8 & .6 & .7 & \text { UER }\end{array}$

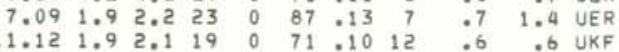
$\begin{array}{llllllllllll}7.82 & 3.2 & 3.6 & 23 & 0 & 129 & .11 & 9 & .7 & 1.0 & \text { MER }\end{array}$

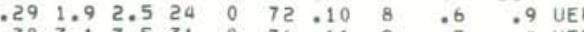

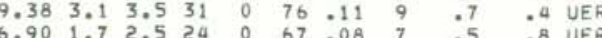

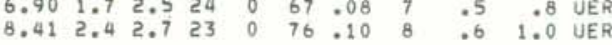
$\begin{array}{lllllllllll}.16 & 2.7 & 3.3 & 25 & 0 & 82 & .13 & 9 & .8 & 1.1 & \text { UER } \\ 6.34 & 2.0 & 2.4 & 25 & 0 & 94 & .14 & 6 & .8 & 1.7 & \text { UER }\end{array}$ $7.88 \quad 2.92 .9$
28

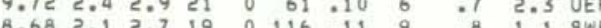
$\begin{array}{lllllllllll}8.26 & 2.2 & 2.7 & 25 & 0 & 91 & .11 & 9 & .6 & .7 & \text { UER }\end{array}$
HVO EARTHQUAKE SUMMARY LIST

PAGE 12 ORIGIN TIME LAT N
YEAR MON DA HRMN SEC DEG MIN DEG MIN $\begin{array}{lllllllll}1976 & \text { FEB } 25 & 11 & 2 & 43.48 & 19 & 20.21 & 155 & 17.03\end{array}$ $251957 \quad 6.621920 .08 \quad 155 \quad 8.65$

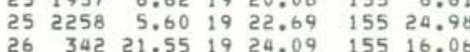
$\begin{array}{lllllll}26 & 1423 & 37.39 & 19 & 24.01 \quad 155 & 15.62\end{array}$ $2618 \quad 3 \quad 54.851919 .29 \quad 15516.45$ $27 \begin{array}{llllll}1135 & 6.58 & 19 & 19.71 & 155 & 15.65\end{array}$

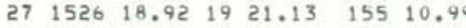
$\begin{array}{lllllll}27 & 1758 & 35.41 & 19 & 21.90 & 155 & 7.05\end{array}$

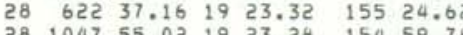

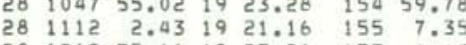
$28121255.611923 .21 \quad 155 \quad 4.60$

$\begin{array}{lllllll}28 & 1526 & 58.09 & 19 & 18.04 & 155 & 26.26\end{array}$ $\begin{array}{lllllll}28 & 1738 & 35.85 & 19 & 19.18 & 155 & 12.07 \\ 28 & 2037 & 49.12 & 19 & 21.89 & 155 & 15.09\end{array}$ $28235525.891922 .42 \quad 155 \quad 6.36$

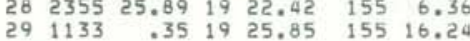

$\begin{array}{lllllll}29 & 1446 & 11.92 & 19 & 17.73 & 155 & 21.09\end{array}$

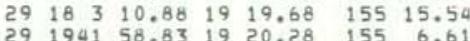

MAR $132544.791923 .62 \quad 155 \quad 3.1$

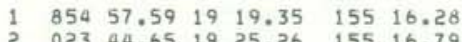
$\begin{array}{lllllll}2 & 023 & 44.65 & 19 & 25.26 & 155 & 16.79\end{array}$

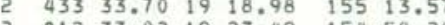

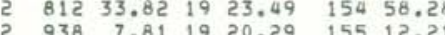

$\begin{array}{lllllll}2 & 946 & 27.07 & 19 & 20.77 & 155 & 11.14\end{array}$ 2 $10 \quad 6 \quad 32.52 \quad 19 \quad 19.25 \quad 155 \quad 11.91$

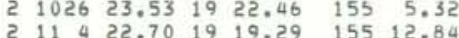
$\begin{array}{llllllll}2 & 11 & 4 & 22.70 & 19 & 19.29 & 155 & 12.84 \\ 2 & 1114 & 30.99 & 19 & 20.10 & 155 & 13.21\end{array}$ $\begin{array}{lrrrrrr}2 & 1150 & 12.64 & 19 & 20.76 & 155 & 11.77 \\ 2 & 1228 & 2.97 & 19 & 20.92 & 155 & 13.31\end{array}$ $\begin{array}{lllllll}2 & 1343 & 42.38 & 19 & 17.80 & 155 & 23.66\end{array}$ $\begin{array}{lllllll}2 & 1452 & 39.66 & 19 & 19.36 & 155 & 16.43 \\ 2 & 1531 & 21.05 & 19 & 19.93 & 155 & 16.87\end{array}$ $\begin{array}{lllllll}\text { 2 } 1634 & 16.63 & 19 & 21.54 & 155 & 13.53\end{array}$ $\begin{array}{llllll}1750 & 45.08 & 19 & 19.09 & 155 & 13.93\end{array}$ $\begin{array}{lllllll}2 & 1814 & 27.57 & 19 & 19.43 & 155 & 15.06 \\ 2 & 1832 & 56.05 & 19 & 19.01 & 155 & 13.01\end{array}$

$2 \quad 1852 \quad 25.90 \quad 1920.05 \quad 155 \quad 17.04$ $2193944.97 \quad 1920.89 \quad 155 \quad 10.44$

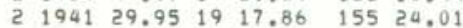

DEPTH AMP OUR NR NSAP RMS MIN ERH ERZ
KM MAG MAG NR NS DEG SEC DIS KM KM REMK $\begin{array}{lllllllllll}7.23 & 1.8 & 2.4 & 21 & 0 & 81 & .13 & 5 & .8 & 1.2 & K O A\end{array}$

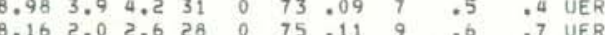

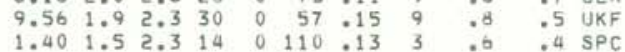

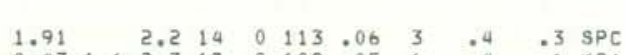
$\begin{array}{lllllllllll}1.91 & 2.2 & 14 & 0 & 113 & .06 & 3 & .4 & .3 & \text { SPC } \\ 8.43 & 1.6 & 2.3 & 17 & 0 & 109 & .05 & 6 & .4 & .6 & \text { KOA }\end{array}$

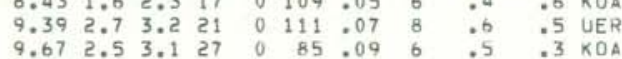

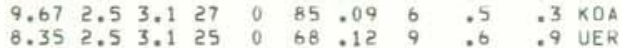
$\begin{array}{lllllllllll}8.00 & 2.9 & 3.3 & 28 & 0 & 74 & .09 & 8 & .4 & .7 & \text { UER }\end{array}$

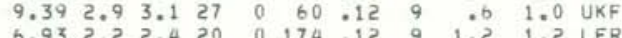

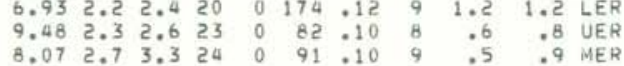
$\begin{array}{lllllllllll}8.14 & 1.9 & 2.5 & 24 & 0 & 95 & .17 & 9 & .9 & 1.6 & \text { HEA } \\ 8.78 & 2.5 & 2.8 & 22 & 0 & 97 & 09 & 7 & .6 & 1.0 & \text { UER }\end{array}$

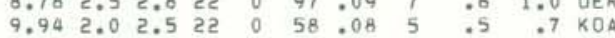
$8.123 .3 \quad 3.625$ 25 69.11 \& $0.6 \quad .8$ UER

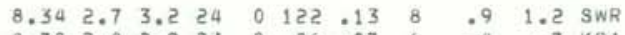

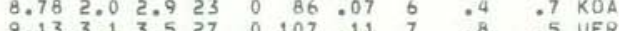

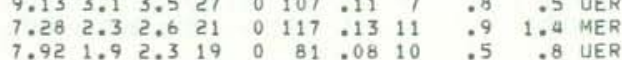

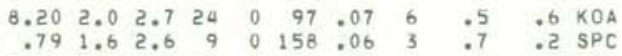

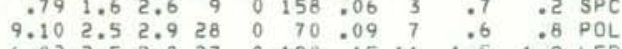

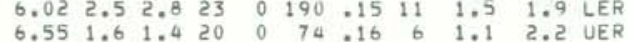
$\begin{array}{llllllllll}8.99 & 1.4 & 18 & 0 & 72 & .07 & 8 & .6 & 1.0 & \text { UER }\end{array}$

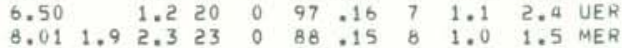

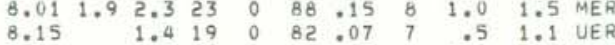

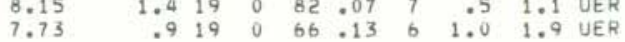
$\begin{array}{lllllllllll}9.21 & 1.7 & 1.8 & 23 & 0 & 70 & .09 & 8 & .6 & 1.0 & \text { UER }\end{array}$

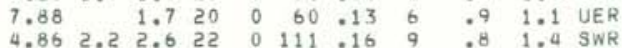

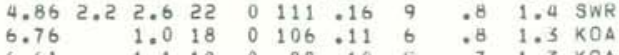

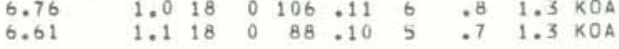

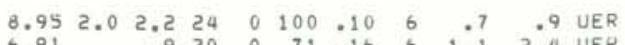
$\begin{array}{lllllllll}6.91 & 9920 & 0 & 71 & .16 & 6 & 1.1 & 2.4 & \text { UER } \\ 8.19 & 1.916 & 0 & 84 & 08 & 76 & .7 & 1.5 & \text { UER }\end{array}$

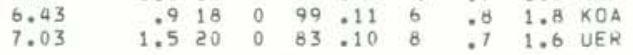
$\begin{array}{lllllllllll}7.45 & 1.5 & 19 & 0 & 84 & .13 & 5 & .7 & 1.1 & \text { KOA } & \end{array}$ $1.291 .92 .224 \quad 0 \quad 109.18$ 9 90 36.9 SWR 
HVO EARTHQUARE SUMMARY LIST

PAGE 13

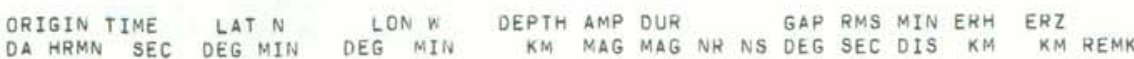

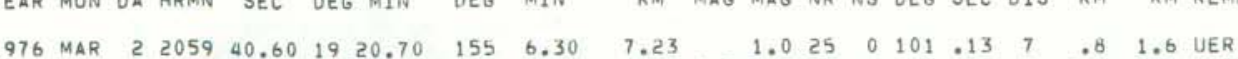

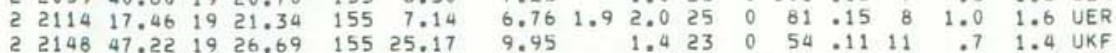

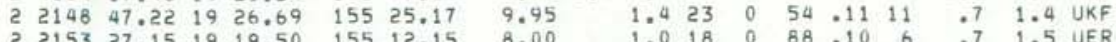

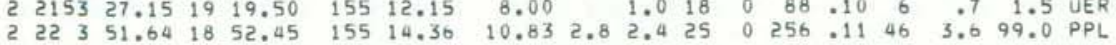

2 $2221 \quad 15.40 \quad 1920.94 \quad 155 \quad 13.07$

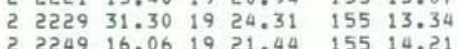

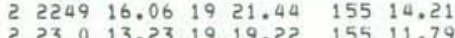

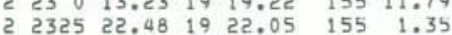

$\begin{array}{lllllll}3 & 045 & 21.56 & 19 & 19.98 & 155 & 8.41\end{array}$

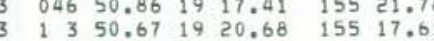
$\begin{array}{lllllll}3 & 351 & 11.79 & 19 & 19.16 & 155 & 15.29 \\ 3 & 420 & 49.25 & 19 & 18.89 & 155 & 21.16\end{array}$

$\begin{array}{lllllll}3 & 428 & 59.39 & 19 & 19.91 & 155 & 18.74\end{array}$ $\begin{array}{rrrrrrr}3 & 440 & 44.10 & 19 & 21.20 & 155 & 6.07 \\ 3 & 530 & 16.50 & 20 & 9.93 & 155 & 15.68\end{array}$ $\begin{array}{llllllll}3 & 6 & 0 & 57.64 & 19 & 25.16 & 155 & 28.11 \\ 3 & 6 & 2 & 6.99 & 19 & 25.06 & 155 & 27.87\end{array}$

$\begin{array}{lllllll}3 & 638 & 49.03 & 19 & 18.63 \quad 155 \quad 11.66\end{array}$ $\begin{array}{llllllll}3 & 726 & 36.53 & 19 & 17.21 & 155 & 22.98\end{array}$ $\begin{array}{lllllll}3 & 934 & 44.56 & 19 & 20.97 & 155 & 2.90 \\ 3 & 952 & 32.24 & 19 & 17.06 & 155 & 22.64\end{array}$

$3 \quad 1249 \quad 50.81 \quad 19 \quad 20.06 \quad 155 \quad 7.18$ $\begin{array}{llllllll}3 & 13 & 6 & 10.20 & 19 & 25.61 & 155 & 16.53\end{array}$ $32048 \quad 47.28 \quad 1922.24 \quad 155 \quad 5.11$

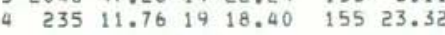
$4 \quad 630 \quad 47.68 \quad 1924.25 \quad 155 \quad 16.02$

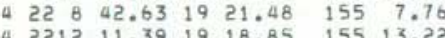

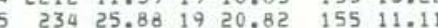
$5 \quad 316 \quad 52.46 \quad 19 \quad 24.76 \quad 155 \quad 7.80$ $\begin{array}{lllllll}5 & 548 & 14.28 & 19 & 20.96 & 155 & 9.54\end{array}$ $\begin{array}{lllllll}5 & 710 & 6.93 & 19 & 24.11 & 155 & 16.01 \\ 5 & 913 & 28.34 & 19 & 20.04 & 155 & 8.41\end{array}$ $5114251.2919 \quad 16.68 \quad 155232.97$

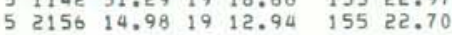

$\begin{array}{lllllll}6 & 613 & 12.70 & 19 & 22.67 & 155 & 5.92 \\ 6 & 745 & 47.00 & 19 & 19.76 & 155 & 8.71\end{array}$

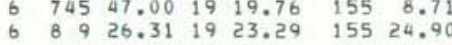
$611495.001921 .23 \quad 15513.35$

$\begin{array}{llllllll}6 & 19 & 3 & 44.53 & 19 & 23.31 & 155 & 16.99\end{array}$ $\begin{array}{lllllll}6 & 2338 & 34.21 & 19 & 22.37 & 155 & 3.94\end{array}$ $\begin{array}{llllllllll}7.38 & .9 & 21 & 0 & 59 & .14 & 6 & .9 & 1.7 & \text { UER } \\ 9.95 & 9 & 19 & 0 & 88 & .09 & 6 & 97 & 1.0 & \text { GLN }\end{array}$

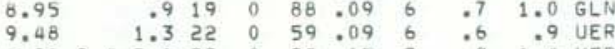

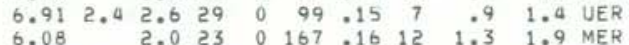
$\begin{array}{lllllllllll}8.38 & 1.1 & 19 & 0 & 80 & .08 & 10 & .5 & .9 & \text { UER } \\ 1.99 & 1.5 & 20 & 2 & 123 & .17 & 9 & .9 & 1.6 & \text { SWR }\end{array}$

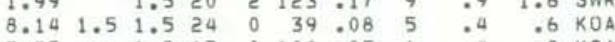
$\begin{array}{lllllllllll}7.27 & 1.2 & 17 & 0 & 108 & .05 & 6 & .4 & .9 & \text { KOA } \\ 7.02 & 1.9 & 2.2 & 26 & 0 & 103 & .15 & 7 & .8 & 1.7 & \text { SWR }\end{array}$ $\begin{array}{llllllllll}28.07 & 1.1 & 23 & 0 & 68 & .08 & 7 & 1.0 & 1.9 & \text { DEP }\end{array}$

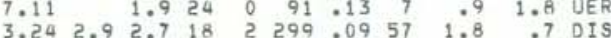
8.82 2. 52.7518 2 299.0957

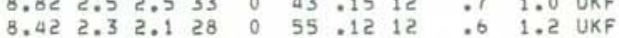
$\begin{array}{llllllllll}7.90 & 1.3 & 22 & 0 & 117 & .14 & 7 & 1.0 & 2.0 & \text { POL }\end{array}$

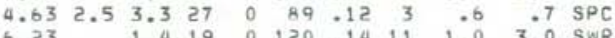

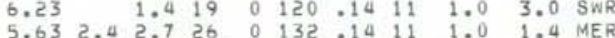

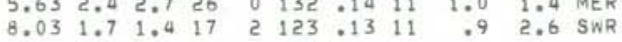
$\begin{array}{llllllllllll}8.98 & 2.6 & 2.8 & 25 & 0 & 103 & .10 & 7 & .7 & .5 & \text { UER }\end{array}$

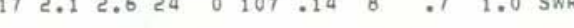
$\begin{array}{llllllllllll}1.63 & 1.8 & 2.6 & 16 & 0 & 114 & .07 & 3 & .3 & .2 & \text { SPC } \\ 8.64 & 2.5 & 3.2 & 27 & 0 & 74 & .10 & 8 & .3 & .2 & 4 P R\end{array}$

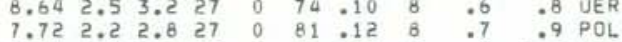

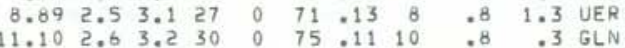
$\begin{array}{llllllllll}7.95 & 2.1 & 2.8 & 26 & 0 & 66 & .14 & 7 & .9 & 1.6\end{array}$

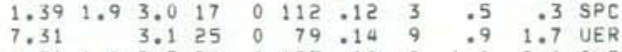

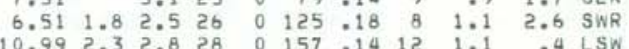

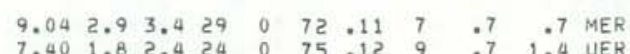

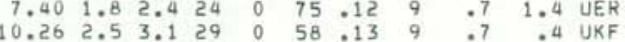

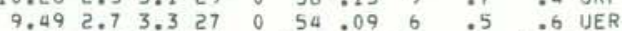

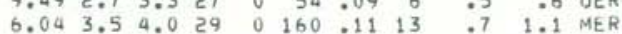
$\begin{array}{lllllllllll}1.90 & 2.6 & 3.4 & 16 & 0 & 62 & .09 & 3 & .5 & .3 & \text { SPC }\end{array}$

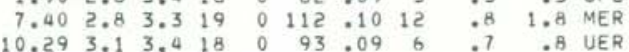
8.632 .93 .2 28 $0107.09 \quad 7 \quad .6095$ UER

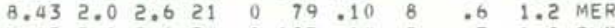

HYO EARTHQUAKE SUMMARY LIST

PAGE 14 ORIGIN TIME LAT N LON W DEPTH AMP DUR GAP RMS MIN ERH ERZ YEAR MON DA HRMN SEC DEG MIN DEG MIN KM MAG MAG NR NS DEG SEC DIS KM KM REMK

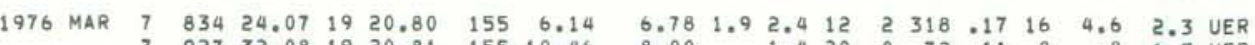

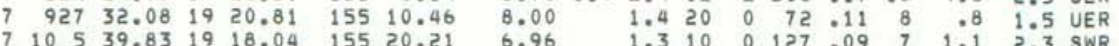

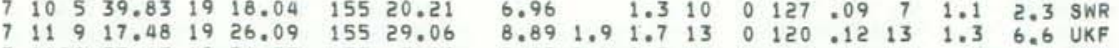

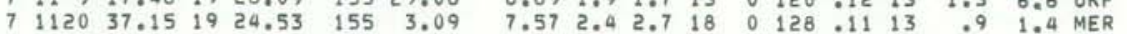

$\begin{array}{llllllllllllllllll}7 & 1123 & 54.42 & 19 & 21.00 & 155 & 10.72 & 8.58 & 2.2 & 2.2 & 17 & 0 & 81 & .07 & 8 & .6 & 1.2 & \text { UER } \\ 7 & 12 & 51.74 & 19 & 20.47 & 155 & 12.47 & 8.27 & 1.3 & 22 & 0 & 69 & .12 & 7 & .8 & 1.6 & \text { UER }\end{array}$

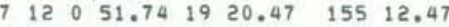
7131955.561923 .5915516 .84

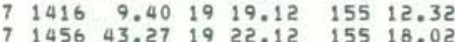

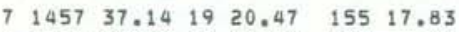

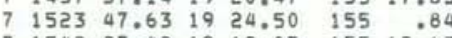

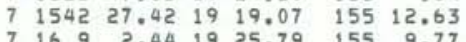
716925.441925 .79 155 9.79

$\begin{array}{lllllll}7 & 1635 & 3.83 & 19 & 23.78 & 155 & 16.93\end{array}$ $\begin{array}{lllllll}7 & 1818 & 56.52 & 19 & 20.54 & 155 & 13.31\end{array}$ $7 \begin{aligned} & 7 \\ & 7\end{aligned} 1825 \quad 1.591926 .59 \quad 15525.21$

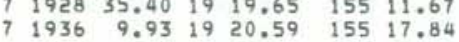

$\begin{array}{lllllll}7 & 1953 & 46.47 & 19 & 17.59 & 155 & 22.53\end{array}$

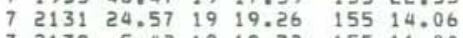
$\begin{array}{lllllll}7 & 2139 & 5.42 & 19 & 19.32 & 155 & 11.80\end{array}$ $\begin{array}{lrrrrrr}7 & 2152 & 1.96 & 19 & 19.43 & 155 & 11.72 \\ 7 & 2231 & 13.16 & 19 & 19.67 & 155 & 7.72\end{array}$

$72234 \quad 32.76 \quad 19 \quad 19.41 \quad 155 \quad 12.95$
7

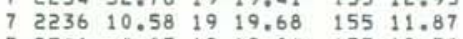
$\begin{array}{llllllll}7 & 2341 & 4.05 & 19 & 19.04 & 155 & 12.58\end{array}$

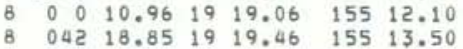

$\begin{array}{lllllll}8 & 047 & 7.83 & 19 & 21.74 & 155 & 14.93\end{array}$ $\begin{array}{llllllll}8 & 117 & 21.98 & 19 & 19.796 & 155 & 18.62 \\ 8 & 136 & 1.39 & 19 & 30.25 & 154 & 50.78\end{array}$ $\begin{array}{lllllll}8 & 243 & 8.58 & 19 & 19.24 & 155 & 15.34\end{array}$

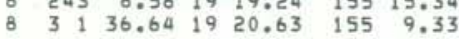

$\begin{array}{lllllll}8 & 322 & 54.48 & 19 & 20.27 & 155 & 8.97\end{array}$ $\begin{array}{lllllll}8 & 352 & 22.10 & 19 & 21.21 & 155 & 3.67 \\ 8 & 4 & 40.64 & 19 & 21.79 & 155 & 2.88\end{array}$

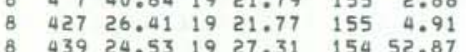
$\begin{array}{lllllll}8 & 446 & 4.17 & 19 & 28.07 & 154 & 54.57\end{array}$ $\begin{array}{lllllllll}8 & 5 & 1 & 36.68 & 19 & 18.71 & 155 & 22.20\end{array}$ $\begin{array}{lllllll}8 & 546 & 51.00 & 19 & 19.34 & 155 & 11.31 \\ 8 & 549 & 33.14 & 19 & 12.91 & 155 & 28.54\end{array}$ $8 \quad 6212.521928 .04 \quad 15454.86$

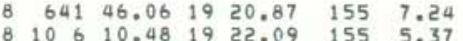

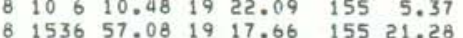
$\begin{array}{lllllllllll}1.67 & 1.4 & 2.0 & 15 & 0 & 68 & .09 & 3 & .4 & .3 & \text { SPC } \\ 5.93 & & 1.1 & 19 & 0 & 94 & .14 & 7 & 1.0 & 2.5 & \text { UER }\end{array}$

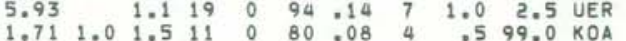

$\begin{array}{llllllllll}6.83 & 1.3 & 17 & 0 & 65 & .08 & 5 & .5 & 1.0 & \text { KOA }\end{array}$

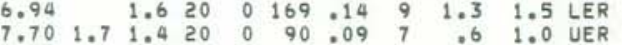
$\begin{array}{lllllllllll}.63 & 1.4 & 23 & 0 & 45 & .13 & 8 & .6 & .9 & 9 \mathrm{GLN}\end{array}$ $\begin{array}{lllllllllllll}1.76 & 1.6 & 2.1 & 14 & 0 & 47 & .06 & 3 & 3 & 3 & \text { SPC }\end{array}$

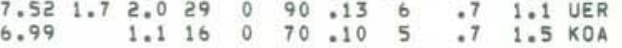

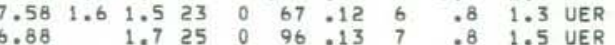

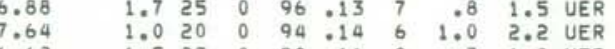
$6.62 \quad 1.5230098 .11 \quad 8 \quad .7$ 1.8 UER

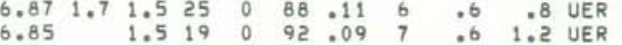

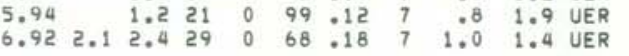

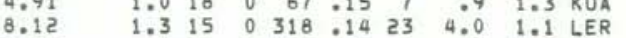
$\begin{array}{lllllllllll}7.47 & 9 & 16 & 0 & 106 & .10 & 6 & .7 & 1.5 & \mathrm{kOA}\end{array}$ $\begin{array}{lllllllll}1.0 & 22 & 0 & 69 & .16 & 8 & 1.1 & 2.0 & \text { UER }\end{array}$ $\begin{array}{llllllllll}7.39 & 1.3 & 24 & 0 & 71 & -11 & 8 & 7 & 1.4 & \text { UER }\end{array}$

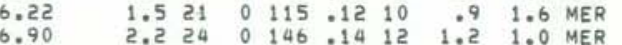
$6.72 \quad 1.323 \quad 0 \quad 90.1399 .8$ 1.4 MER $\begin{array}{llllllllll}6.94 & 1.1 & 18 & 0 & 250 & .16 & 20 & 3,2 & .6 & \text { LER }\end{array}$ $\begin{array}{llllllllllll}5.44 & 1.5 & 18 & 0 & 171 & .20 & 17 & 2.9 & 2.1 & \text { LER }\end{array}$ $\begin{array}{llllllllllll}7.82 & 1.9 & 2.2 & 26 & 0 & 108 & 17 & 9 & 1.0 & 1.3 & \text { SWR } \\ 5.87 & & 1.1 & 21 & 0 & 100 & 11 & 7 & .8 & 1.3 & \text { UER }\end{array}$

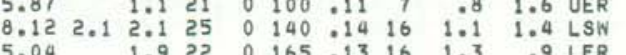

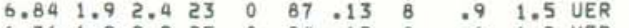

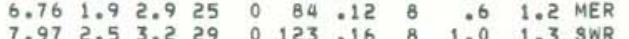

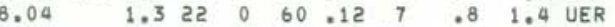
$\begin{array}{lllllllllll}1.3 & 16 & 0 & 71 & .09 & 11 & .9 & .5 & \text { UKF }\end{array}$

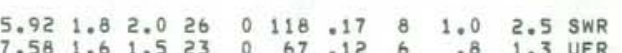
$\begin{array}{lllllllllll}5.93 & 1.3 & 20 & 0 & 78 & .15 & 7 & .9 & 2.3 & \text { UER }\end{array}$ $\begin{array}{lllllllllll}9.20 & 2.1 & 2.7 & 24 & 0 & 59 & .10 & 4 & .5 & .7 & \text { UER }\end{array}$ 
HVO EARTHQUAKE SUMMARY LIST

PAGE 15

ORIGIN TIME LAT N LON W DEPTH AMP OUR GAP RMS MIN ERH ERZ YEAR MON DA HRMN SEC DEG MIN DEg MIN

1976 MAR $9238 \quad 42.37 \quad 1922.28 \quad 155 \quad 5.28$

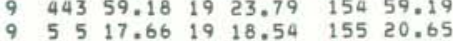

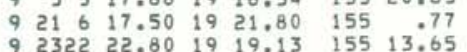
$10 \quad 026 \quad 52.24 \quad 1924.13 \quad 155 \quad 16.11$ $10 \quad 04926.401919 .95 \quad 155 \quad 8.79$ $\begin{array}{lllllll}10 & 613 & 34.98 & 19 & 20.79 & 155 & 13.21\end{array}$

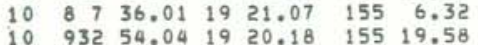
$\begin{array}{lllllll}10 & 952 & 20.49 & 19 & 18.23 & 155 & 23.38\end{array}$ $\begin{array}{lllllll}10 & 1832 & 40.95 & 19 & 20.30 & 155 & 7.13\end{array}$

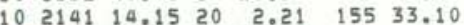

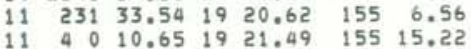

$\begin{array}{llllllll}11 & 4 & 2 & 42.23 & 19 & 21.60 & 155 & 15.45\end{array}$ $1933 \quad 16.86 \quad 1920.50 \quad 155 \quad 13.80$ $11 \quad 1019 \quad 32.18 \quad 1920.34 \quad 155 \quad 8.05$ $111422 \quad 48.08 \quad 1920.93 \quad 15511.24$

$\begin{array}{lllllll}12 & 339 & 5.14 & 19 & 19.64 & 155 & 15.77\end{array}$

$\begin{array}{lllllll}\text { w } 12 & 518 & 5.94 & 19 & 20.04 & 155 & 8.47\end{array}$ $\begin{array}{llllllll}12 & 21 & 1 & 12.47 & 19 & 19.65 & 155 & 11.66 \\ 12 & 2110 & 17.30 & 19 & 30.03 & 154 & 50.74\end{array}$ $\begin{array}{lllllll}13 & 616 & 16.38 & 19 & 8.69 & 155 & 30.14\end{array}$ $\begin{array}{lllllll}13 & 1012 & 14.16 & 19 & 20.19 & 155 & 8.05\end{array}$ $13 \quad 1642 \quad 37.02 \quad 19 \quad 19.15 \quad 15522.00$

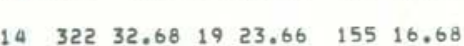
$14 \quad 354 \quad 42.19 \quad 19 \quad 19.33 \quad 155 \quad 12.51$ $141114 \quad 58.13 \quad 1922.94 \quad 15525.02$ $15 \quad 1526 \quad 12.53 \quad 19 \quad 17.91 \quad 15523.54$

$\begin{array}{lllllll}15 & 1532 & 25.41 & 19 & 19.47 & 155 & 12.38 \\ 15 & 1555 & 26.14 & 19 & 17.96 & 155 & 23.60\end{array}$ $15155526.141917 .96 \quad 15523.60$ $\begin{array}{lllllll}15 & 1953 & 2.46 & 19 & 22.79 & 155 & 26.64\end{array}$ $16 \quad 12232.6719 \quad 19.65 \quad 155 \quad 7.51$

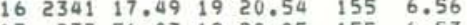

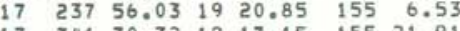
$17 \quad 34130.321917 .1515521 .91$ $17115828.691920 .06 \quad 155 \quad 12.10$

$\begin{array}{lllllll}17 & 1936 & 18.08 & 19 & 19.83 & 155 & 12.36\end{array}$

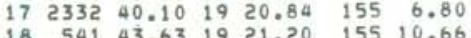
$\begin{array}{llllllllll}0.91 & 2.5 & 3.3 & 28 & 0 & 77 & .15 & 8 & .8 & 1.2 \\ 0 E R\end{array}$

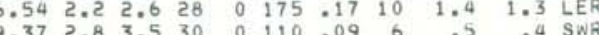

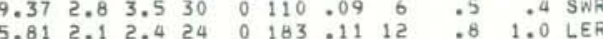

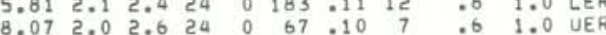

$\begin{array}{lllllllllll}1.45 & 1.6 & 2.5 & 13 & 0 & 110 & .08 & 3 & .4 & .3 & \text { SPC }\end{array}$

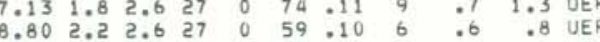
$\begin{array}{llllllllll}8.71 & 2.1 & 2.8 & 26 & 0 & 93 & .09 & 7 & .6 & .6 \\ 7 & .6 & \text { UER }\end{array}$

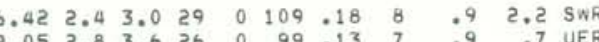
$\begin{array}{lllllllllll}6.35 & 3.1 & 2.3 & 33 & 0 & 191 & 14 & 30 & 1.4 & 3.9 & 0 \mathrm{KOH}\end{array}$

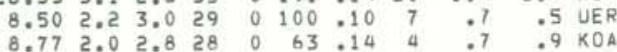
$\begin{array}{llllllllllll}8.98 & 3.1 & 3.6 & 25 & 0 & 67 & .11 & 5 & .7 & .8 & \text { KOA }\end{array}$

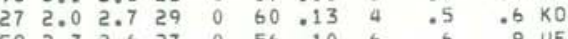
$\begin{array}{lllllllllll}8.59 & 2.3 & 2.6 & 27 & 0 & 56 & .10 & 6 & .6 & .9 & .9\end{array}$

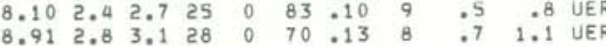
$\begin{array}{lllllllllll}34.35 & 2.9 & 2.8 & 31 & 0 & 88 & .10 & 6 & .9 & 1.6 & \text { DEP }\end{array}$

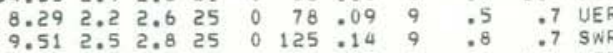
$\begin{array}{lllllllllll}7.41 & 2.2 & 2.8 & 23 & 0 & 90 & .09 & 6 & .6 & 1.1 & \text { UER } \\ 9.18 & 3.3 & 3.4 & 12 & 1 & 269 & .13 & 29 & 3.3 & 1.3 & \text { LER }\end{array}$ \begin{tabular}{llllllllll}
1.26 & 3.5 & 3.5 & 28 & 3 & 156 & .13 & 24 & 1.0 & .4 \\
\hline
\end{tabular}

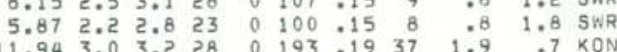

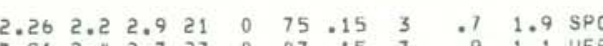
$\begin{array}{lllllllllll}7.51 & 2.4 & 2.7 & 27 & 0 & 87 & .15 & 7 & .9 & 1.1 & \text { UER } \\ 7.89 & 2.2 & 2.3 & 24 & 0 & 57 & 13 & 9 & 9 & 1.4 & \text { UKF }\end{array}$

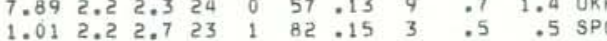

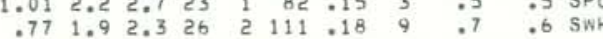
$\begin{array}{lllllllllll}6.93 & 2.2 & 2.6 & 24 & 0 & 86 & 14 & 7 & .9 & 1.4 & \text { UER }\end{array}$ $\begin{array}{llll}9 & 9 & 1.0 & \text { SN }\end{array}$ $\begin{array}{rrrrrrrrrrr}9.63 & 2.0 & 2.3 & 26 & 0 & 51 & .13 & 12 & .6 & .5 & \text { UKF } \\ 9.27 & 2.8 & 3.0 & 27 & 0 & 70 & 12 & 8 & .7 & .7 & \end{array}$ 8.942 .22 .8 21 0103.10 8 9 9 6 UER $\begin{array}{rrrrrrrrrrr}7.92 & 2.1 & 2.9 & 27 & 0 & 102 & 11 & 6 & .6 & .9 & \text { UtR } \\ 8.75 & 2.2 & 2.9 & 27 & 0 & 96 & : 13 & 7 & : 0 & .8 & \text { UER }\end{array}$

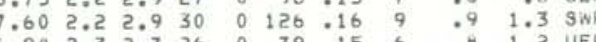

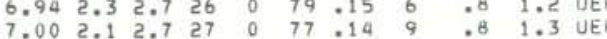

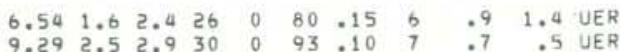
$\begin{array}{lllllllllll}9.29 & 2.5 & 2.9 & 30 & 0 & 93 & .10 & 7 & .7 & .5 & \text { UER } \\ 7.86 & 1.8 & 2.6 & 26 & 0 & 68 & .13 & 9 & .8 & 1.1 & \text { UER }\end{array}$

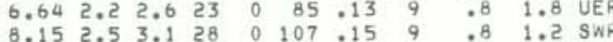

HVO EARTMOUAKE SUMMARY LIST

PAGE 16 ORIGIN TIME LAT N LON W DEPTH AMP DUR GAP RMS MIN ERH ERZ REMK TEAR MON ORI HRMN SEC DEG MIN DEG MIN SPC

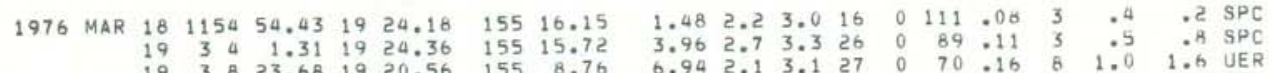

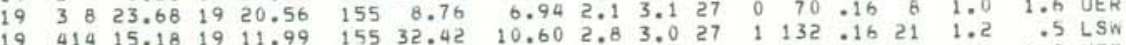

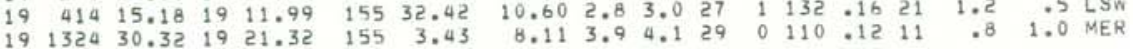
$\begin{array}{lllllll}19 & 1555 & 6.57 & 19 & 20.90 & 155 & 2.96\end{array}$ $20 \quad 3536.301918 .74 \quad 1559.16$

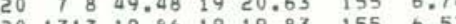
$20131319.8619 .9 .83 \quad 155 \quad 6.53$ $21 \quad 931 \quad 33.54 \quad 19 \quad 22.23 \quad 155 \quad 15.26$ $\begin{array}{lllllll}21 & 1358 & 016 & 19 & 20.90 & 155 & 11.30\end{array}$ $\begin{array}{lllllll}21 & 1745 & 48.58 & 19 & 20.34 & 155 & 6.70\end{array}$ $21203124.601920 .22 \quad 155 \quad 8.61$

$\begin{array}{lllllll}21 & 2355 & 24.10 & 19 & 19.32 & 155 & 11.82\end{array}$ $\begin{array}{lllll}94 & 19 & 22.32 & 155 & 5.32\end{array}$ $\begin{array}{lllllll}22 & 727 & 41.74 & 19 & 17.16 & 155 & 21.73\end{array}$

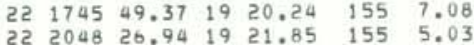

$22 \quad 211126.71 \quad 1921.14 \quad 15520.81$

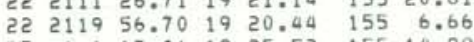
$\begin{array}{llllllll}23 & 6 & 1 & 17.06 & 19 & 25.52 & 155 & 14.99\end{array}$ $\begin{array}{lllllll}23 & 1438 & 11.08 & 19 & 19.83 & 155 & 11.53 \\ 23 & 156 & 3.24 & 19 & 18.82 & 155 & 18.38\end{array}$ $\begin{array}{lllllll}23 & 1623 & 53.31 & 19 & 21.35 & 155 & 7.69\end{array}$ $23 \quad 1725 \quad 57.511922 .95$ 155 14.00 $\begin{array}{lllllll}24 & 418 & 33.99 & 19 & 20.55 & 155 & 6.48\end{array}$ $\begin{array}{lllllll}24 & 1049 & 4.40 & 19 & 17.72 & 155 & 21.25 \\ 24 & 1218 & 9.85 & 19 & 22.54 & 155 & 13.05\end{array}$ $\begin{array}{lllllll}24 & 1533 & 14.87 & 19 & 23.04 & 155 & 4.42\end{array}$ $24 \quad 172234.35 \quad 1919.63 \quad 15511.55$

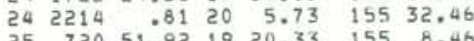
72051.921920 .33 155 8.46 $25 \quad \begin{array}{lllllll}18 & 8 & 45.75 & 19 & 22.26 & 155 & 5.09\end{array}$ $250457.031918 .01 \quad 155 \quad 15.30$ $\begin{array}{lllllllll}26 & 844 & 47.75 & 19 & 20.67 & 155 & 15.30 & \end{array}$ $\begin{array}{lllllll}26 & 1050 & 39.98 & 19 & 21.34 & 155 & 7.68\end{array}$

$\begin{array}{lllllll}26 & 1356 & 31.39 & 19 & 19.45 \quad 155 & 16.49\end{array}$ $\begin{array}{lllllll}26 & 1444 & 25.45 & 19 & 23.11 & 155 & 17.0\end{array}$ $261830 \quad 46.82 \quad 1921.97 \quad 15515.07$ $\begin{array}{lllllll}27 & 932 & 52.58 & 19 & 22.03 & 155 & 6.0\end{array}$

$\begin{array}{lllllll}27 & 933 & 41.63 & 19 & 21.92 \quad 155 \quad 6.78\end{array}$ $\begin{array}{rrrrrrr}27 & 2248 & 53.85 & 19 & 19.92 & 155 & 10.36 \\ 28 & 927 & 53.31 & 19 & 18.76 & 155 & 22.37\end{array}$ $\begin{array}{lllllllllll}6.62 & 2.4 & 2.8 & 25 & 0 & 129 & .11 & 11 & .8 & 1.0 & \text { MER }\end{array}$

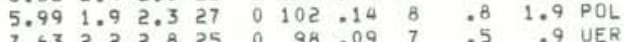

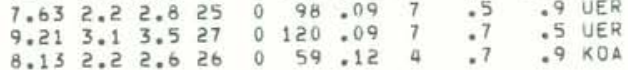
$\begin{array}{lllllllllll}9.78 & 3.3 & 3.6 & 20 & 0 & 55 & 111 & 4 & .8 & 1.6 & K O A\end{array}$

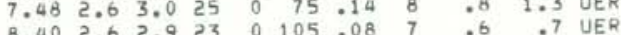

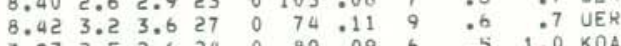

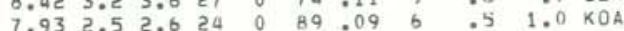

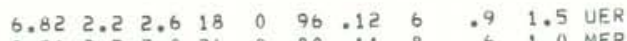

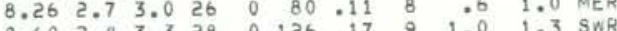

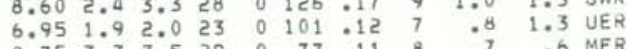

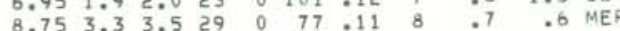

$\begin{array}{lllllllllll}29.44 & 2.4 & 2.4 & 29 & 0 & 63 & .12 & 6 & 1.0 & 2.0 & \text { DEP }\end{array}$

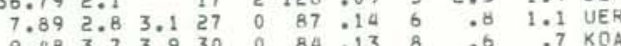
$\begin{array}{lllllllllll}7.08 & 1.8 & 2.7 & 26 & 0 & 76 & .12 & 8 & .7 & 1.0 & \text { UER }\end{array}$

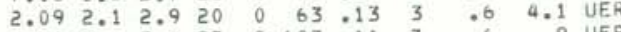

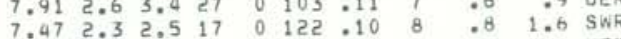

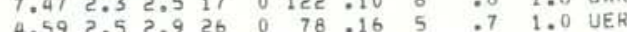

$\begin{array}{lllllllllll}7.48 & 2.6 & 3.0 & 20 & 0 & 95 & .12 & 10 & .8 & 1.5 & \text { MER }\end{array}$

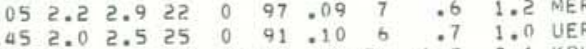

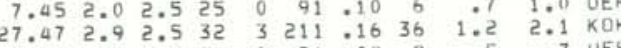

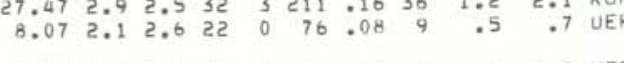
$\begin{array}{lllllllllll}8.57 & 2.7 & 3.1 & 25 & 0 & 80 & .11 & 8 & .6 & 1.0 & \text { MEF }\end{array}$

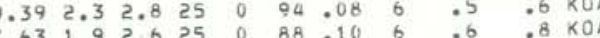

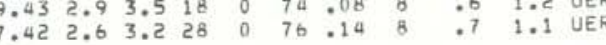
$\begin{array}{lllllllllll}8.75 & 2.6 & 3.4 & 24 & 0 & 97 & .10 & 6 & .6 & .9 & \text { KOA }\end{array}$

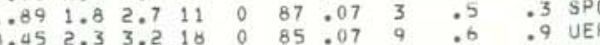

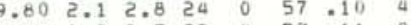

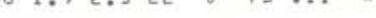
$\begin{array}{lllllllllll}7.75 & 1.9 & 2.7 & 23 & 0 & 75 & .11 & 8 & .7 & 1.5 & \text { UER }\end{array}$

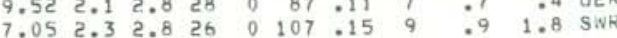

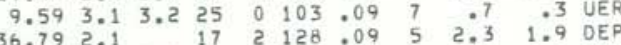

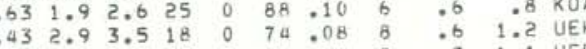




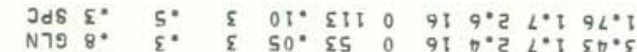

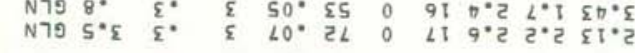

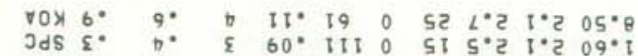

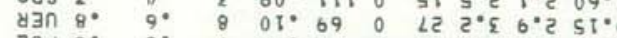

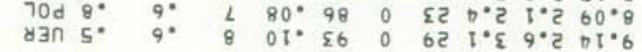

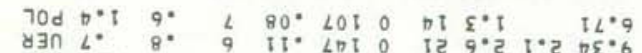

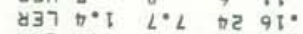

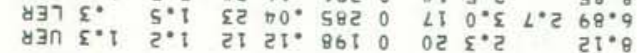

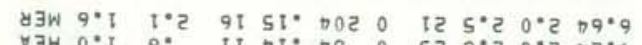

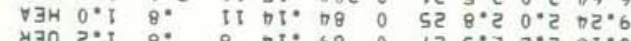

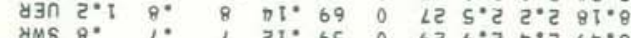

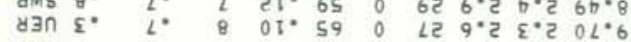

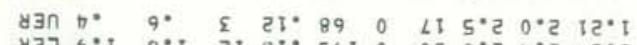

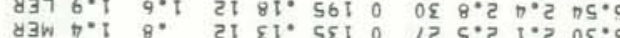

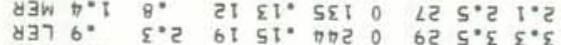

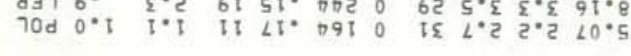

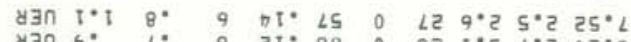

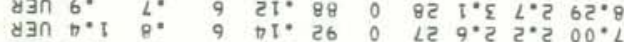

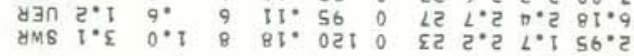

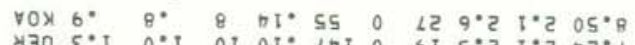

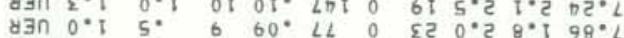

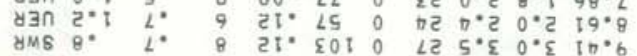

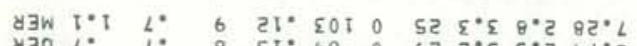

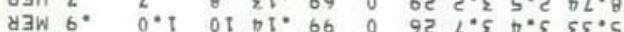
nOX $9^{\circ}$ त.

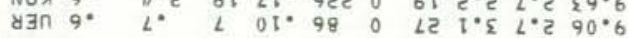

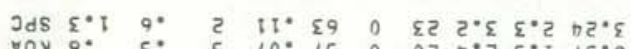

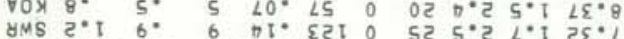

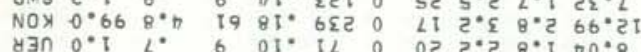

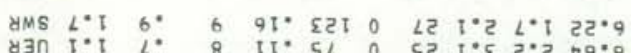

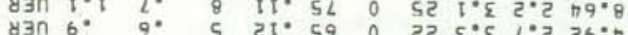
प्र $378^{\circ} 18^{\circ}$.

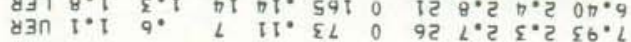

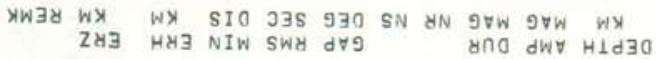
$81398 \mathrm{~d}$

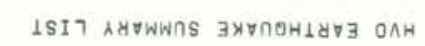

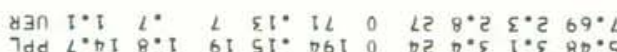

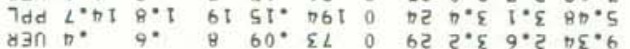

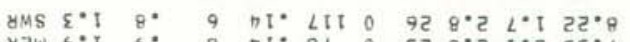

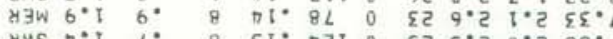

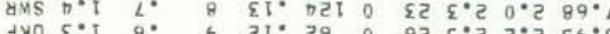

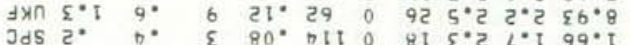

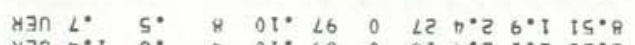

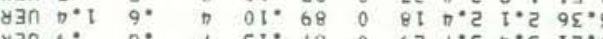

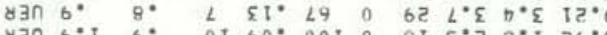

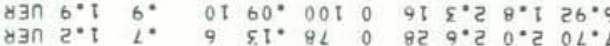

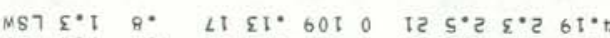

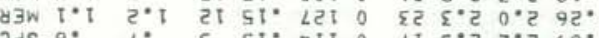

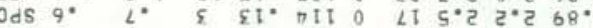

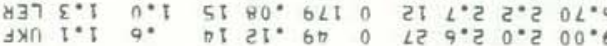

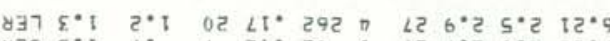

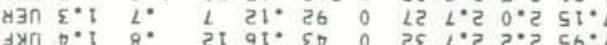

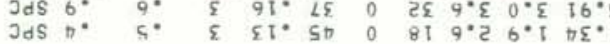

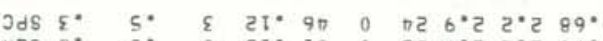

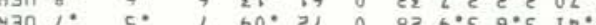

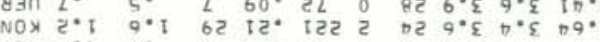

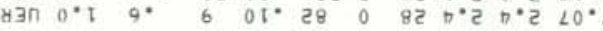

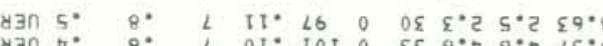

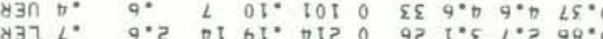

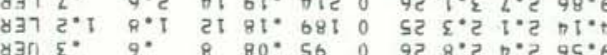

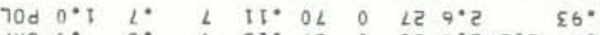

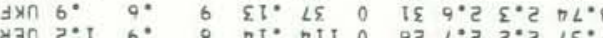

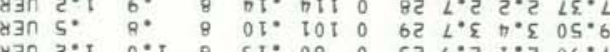

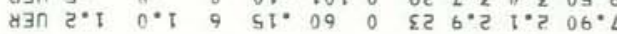

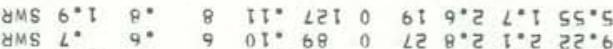

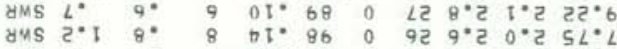

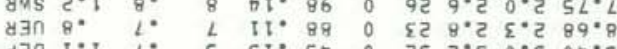

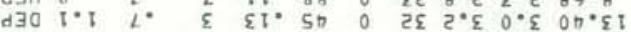

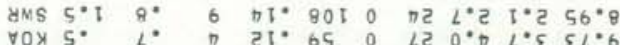

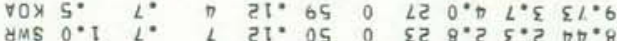

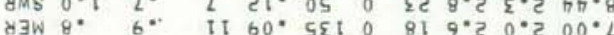

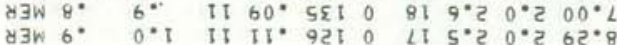

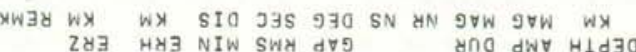

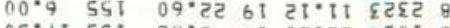

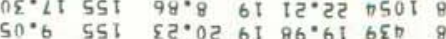

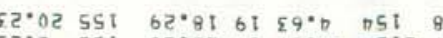

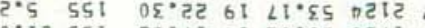

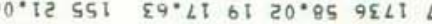

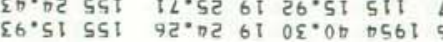

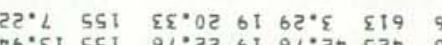

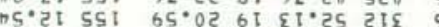

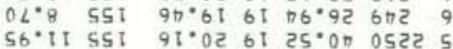

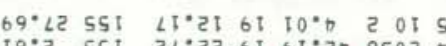

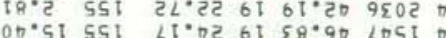

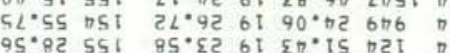
एह. 25 DST $6 \varepsilon^{\circ} 65$ 6I $90^{\circ} 55$ OHLT ह

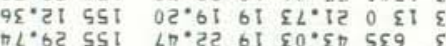

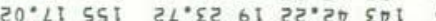

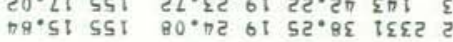

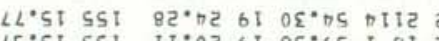
25. हो 5ड 11.02 6ा 85.65 र 81

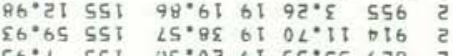

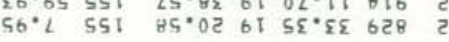
$\begin{array}{lllllll}60 \% & 551 & 97^{\circ} 05 & 61 & 15.95 & 858 & 2 \\ 05.9 & 551 & 59.05 & 61 & 05.9 & 718 & 5\end{array}$

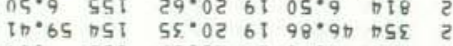

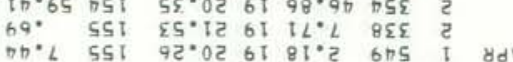

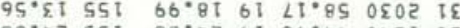
कर है.

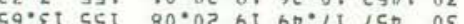

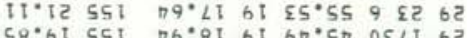

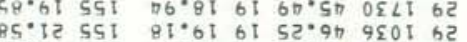

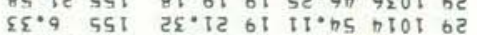

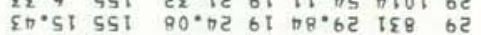

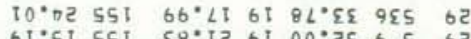

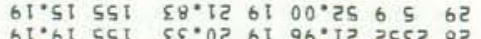
6.

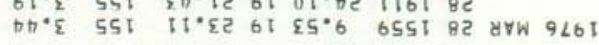
NIW 930 NIW 930 J3S NWYH VO NOW Y Y $\angle T \quad 3980$

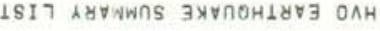


$\begin{array}{llllll}\text { ORIGIN TIME } & \text { LAT N } & \text { LON } W & \text { DEPTH AMP DUR } & \text { GAP RMS MIN ERH ERZ } \\ \text { DA HRMN SEC DEG MIN DEG MIN } & \text { KM MAG MAG NR NS DEG SEC DIS KM KM REMK }\end{array}$

1976 APR $18 \quad 2312 \quad 14.03 \quad 1921.00 \quad 155 \quad 13.19$

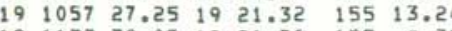
$191133 \quad 38.071921 .78 \quad 155 \quad 4.39$

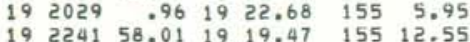

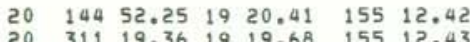
$20 \quad 311 \quad 19.36 \quad 19 \quad 19.68 \quad 155 \quad 12.43$ $\begin{array}{lllllll}20 & 711 & 3.11 & 19 & 21.70 & 155 & 15.25\end{array}$

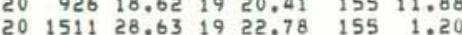

$\begin{array}{lllllll}20 & 1925 & 44.68 & 19 & 21.26 & 155 & 6.34\end{array}$ $20 \quad 1934 \quad 15.46 \quad 19 \quad 19.95 \quad 155 \quad 11.82$ $\begin{array}{lllllll}21 & 1423 & 10.63 & 19 & 19.69 & 155 & 10.52\end{array}$

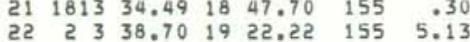
$\begin{array}{llllllll}22 & 4 & 5 & 10.35 & 19 & 19.58 \quad 155 \quad 8.18\end{array}$

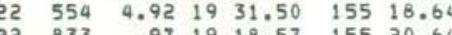
$\begin{array}{llllll}833 & 097 & 19 & 18.57 & 155 & 20.64\end{array}$

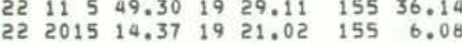
$\begin{array}{lllllll}23 & 145 & 15.82 & 19 & 21.40 & 155 & 7.56\end{array}$

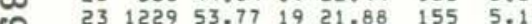

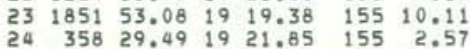
$24 \quad 1754 \quad 11.09 \quad 19 \quad 19.92 \quad 155 \quad 19.70$ $\begin{array}{lllllll}24 & 2055 & 31.31 & 19 & 20.88 & 155 & 11.18\end{array}$ $\begin{array}{lllll}25 & 1449 \quad 11.98 & 19 & 16.55 \quad 155 & 21.85\end{array}$

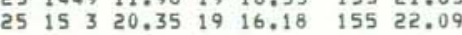
$\begin{array}{lllllll}25 & 1632 & 28.03 & 19 & 22.87 & 155 & 4.63\end{array}$ $25163958.83 \quad 1923.15 \quad 155 \quad 3.63$ $26 \quad 637 \quad 17.42 \quad 1919.99 \quad 15512.45$ $\begin{array}{llllllll}26 & 8 & 4 & 4.58 & 19 & 23.32 & 155 & 16.87\end{array}$

$\begin{array}{lllllll}26 & 1142 & 30.62 & 19 & 16.87 & 155 & 21.71\end{array}$ $\begin{array}{lllllll}26 & 2144 & 41.56 & 19 & 23.26 & 155 & 16.96\end{array}$ $77530.271920 .12 \quad 155 \quad 6.66$ $27 \quad 1317 \quad 51.64 \quad 19 \quad 17.03 \quad 155 \quad 22.50$

$\begin{array}{lllllll}27 & 1340 & 12.13 & 19 & 24.37 & 155 & 15.72\end{array}$

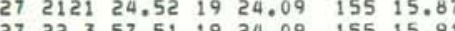

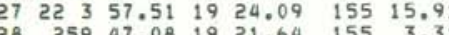

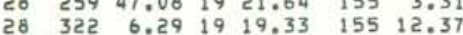

$\begin{array}{lllllll}28 & 1355 & 2.65 & 19 & 19.84 & 155 & 7.05\end{array}$ $\begin{array}{lllllll}28 & 1611 & 50.88 & 19 & 23.69 & 154 & 59.17 \\ 28 & 2148 & 17.39 & 19 & 20.83 & 155 & 7.98\end{array}$ \begin{tabular}{llllllllll}
9.17 & 2.3 & 3.0 & 23 & 0 & 58 & .11 & 6 & .7 & 1.2 \\
\hline
\end{tabular}

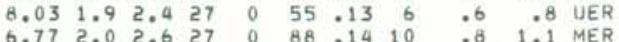

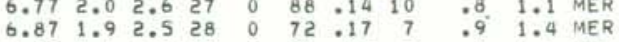

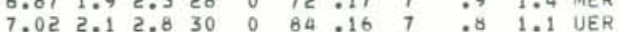

$\begin{array}{llllllllll}7.29 & 2.6 & 3.3 & 29 & 0 & 71 & .15 & 7 & .8 & 1.2 \\ 0.06 R\end{array}$ $\begin{array}{lllllllllll}7.06 & 2.1 & 2.7 & 29 & 0 & 82 & .12 & 6 & .6 & .9 & \text { UER } \\ 9.50 & 3.5 & 3.7 & 31 & 0 & 60 & .11 & 4 & .5 & .5 & \text { KOA }\end{array}$

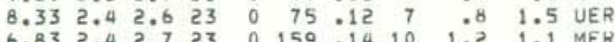

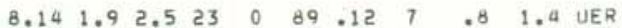

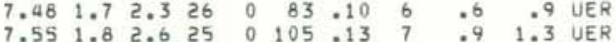

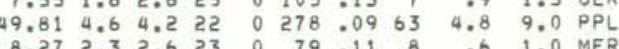

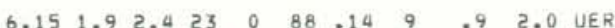

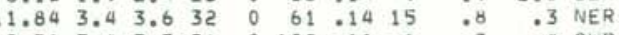

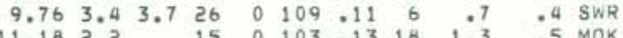

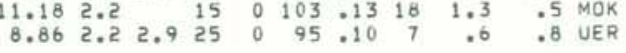
$\begin{array}{llllllllll}6.54 & 2.2 & 2.4 & 24 & 0 & 76 & .13 & 8 & .8 & 1.9 \\ 9.22 & 2.3 & 2.9 & 23 & 0 & 78 & .10 & 8 & .7 & \\ 9.9 & \text { MER }\end{array}$

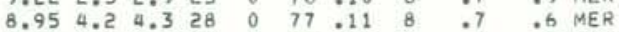

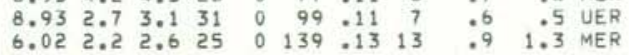
$\begin{array}{lllllllllll}8.31 & 2.3 & 3.0 & 26 & 0 & 64 & .13 & 6 & .6 & .8 & \text { SWR }\end{array}$

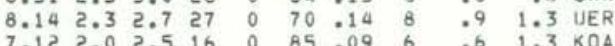

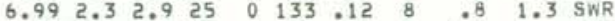

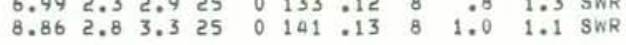
$\begin{array}{lllllllllll}7.52 & 2.3 & 2.6 & 26 & 0 & 91 & .13 & 9 & .7 & 1.1 & \text { MER }\end{array}$

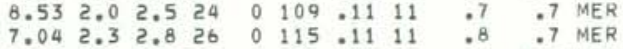

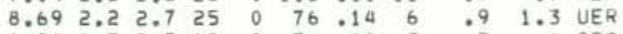

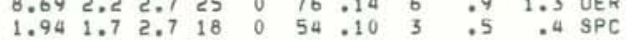
$\begin{array}{rrrrrrrrrrr}5.40 & 3.1 & 3.8 & 26 & 0 & 44 & .10 & 5 & .4 & .6 & \text { SPC } \\ 9.25 & 2.6 & 2.9 & 26 & 0 & 110 & .10 & 7 & : 7 & .6 & \text { UER }\end{array}$

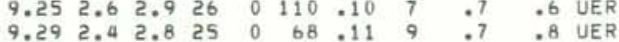

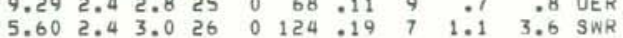

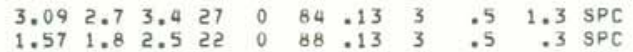

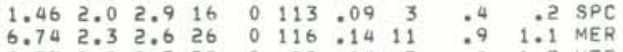

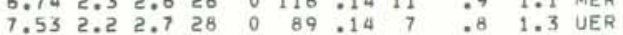

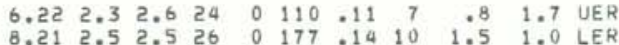

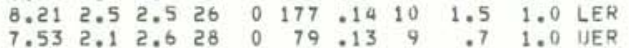
$\begin{array}{llllllllllll}7.40 & 2.2 & 2.9 & 27 & 0 & 130 & .14 & 9 & .9 & 1.3 & \text { SWR }\end{array}$
ORIGIN TIME LATN LON W

TEAR MON DA HRMN SEC DEG MIN DEG MIN

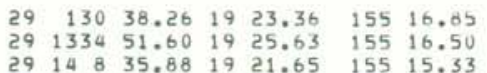

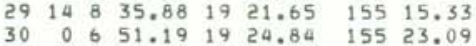
$\begin{array}{llllllll}30 & 0 & 6 & 51.19 & 19 & 24.84 & 155 & 23.09 \\ 30 & 537 & 36.24 & 19 & 25.97 & 155 & 16.48\end{array}$

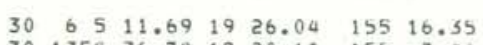

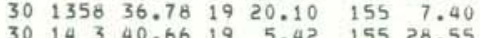

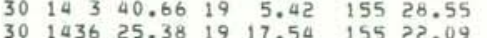
$\begin{array}{lllllll}30 & 1436 & 25.38 & 19 & 17.54 & 155 & 22.09 \\ 30 & 2143 & 34.73 & 19 & 22.50 & 155 & 30.15\end{array}$ $\begin{array}{llllll}30 & 2145 & 23.93 & 19 & 21.53 & 155\end{array}$ $\begin{array}{lllllll}30 & 2318 & 29.50 & 19 & 19.31 & 155 & 12.38\end{array}$

MAY 1 $02454.37 \quad 1926.11 \quad 154 \quad 55.82$

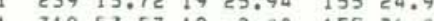
26.89

$\begin{array}{lllllll}1 & 322 & 34.95 & 19 & 5.55 & 155 & 27.48\end{array}$

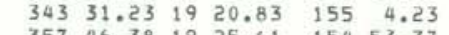

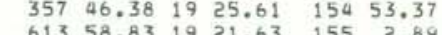
$\begin{array}{rrrrrr}613 & 58.83 & 19 & 21.63 & 155 & 2.84 \\ 854 & 1.01 & 19 & 18.86 & 155 & 13.60\end{array}$ $\begin{array}{lllllll}1 & 921 & 52.26 & 19 & 23.41 & 155 & 17.11\end{array}$ 1437
1
1

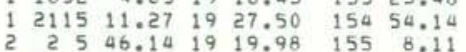

$\begin{array}{lllllll}2 & 952 & 8.66 & 19 & 21.13 & 155 & 10.78\end{array}$

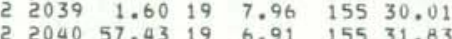
$\begin{array}{lllllll}3 & 156 & 48.39 & 19 & 19.88 & 155 & 7.29 \\ 3 & 158 & 49.07 & 19 & 22.40 & 155 & 7.01\end{array}$

$\begin{array}{lllllllll}3 & 7 & 8 & 12.59 & 19 & 22.35 & 155 & .97\end{array}$ $\begin{array}{lllllll}3 & 1120 & 58.36 & 19 & 20.08 & 155 & 12.74 \\ 3 & 155 & 28.83 & 19 & 18.06 & 155 & 16.15\end{array}$

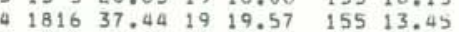

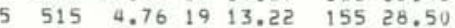

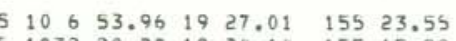
$51832 \quad 20.28 \quad 1924.14 \quad 155 \quad 15.99$ 5232614.791926 .04155 s.18 $\begin{array}{lllllll}6 & 026 & 16.34 & 19 & 23.26 & 155 & 3.96\end{array}$

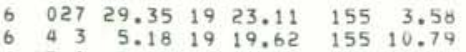
$\begin{array}{llllllll}6 & 17 & 2 & 48.21 & 19 & 19.62 & 155 & 10.79 \\ 6 & 20.29 & 155 & 16.45\end{array}$ $\begin{array}{lllllll}7 & 2136 & 16.96 & 19 & 20.57 & 155 & 7.12\end{array}$

$\begin{array}{lllllll}8 & 727 & 16.08 & 19 & 23.44 & 155 & 16.88\end{array}$

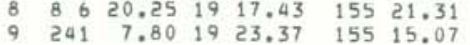

DEPTH AMP DUR GAP RMS MIN ERH ERZ

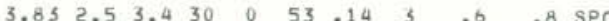

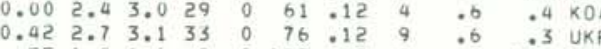

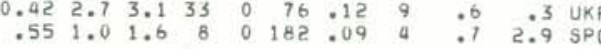

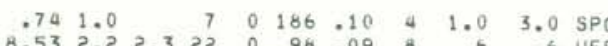

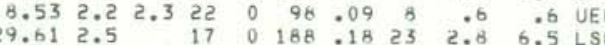

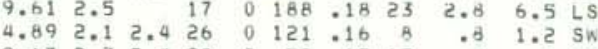

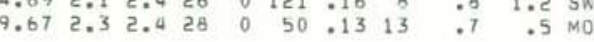

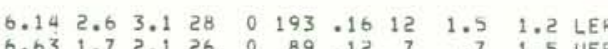

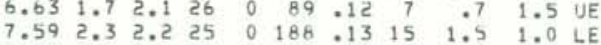

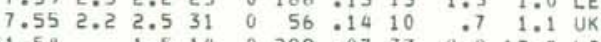
1.54
6.52

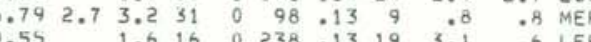

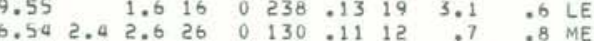
$9.452 .43 .028 \quad 0 \quad 70.097 \quad .5 \quad .6 \mathrm{POL}$ $\begin{array}{llllllllllllllllll}1.53 & 2.8 & 11 & 0 & 58 & .06 & 3 & .4 & .2 & \text { SP }\end{array}$

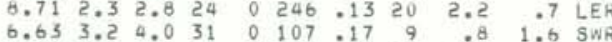

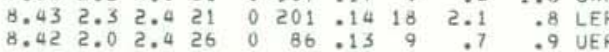
\begin{tabular}{llllllllll}
9.05 & 2.0 & 2.7 & 24 & 0 & 80 & .12 & 9 & .8 & .9 \\
\hline
\end{tabular}

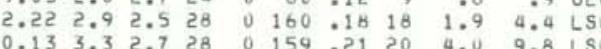

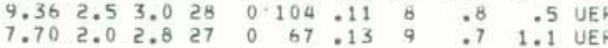
$\begin{array}{lllllllllll}7.30 & 2.6 & 2.8 & 27 & 0 & 169 & .15 & 11 & 1.2 & 1.0 & \text { LEH }\end{array}$

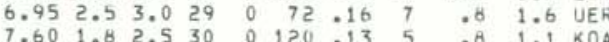

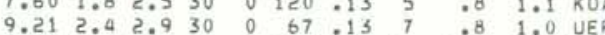
$0.302 .42 .828 \quad 0 \quad 139.16 \quad 16 \quad 1.2 \quad 5$ LS" $\begin{array}{lllllllllll}8.61 & 2.3 & 2.5 & 30 & 0 & 75 & .14 & 11 & .7 & 1.4 & \text { UKF }\end{array}$

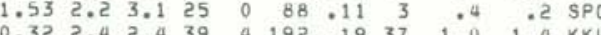
6.432 .93 .431 0 $69.13 \quad 8 \quad .7$ 1.3 UER \begin{tabular}{llllllllll}
8.65 & 2.7 & 3.1 & 33 & 0 & 103 & .12 & 11 & .7 & \\
\hline
\end{tabular}

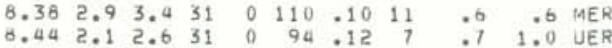

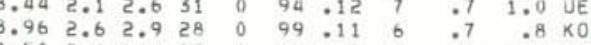

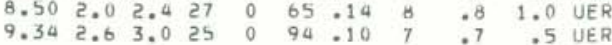

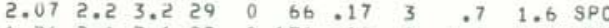

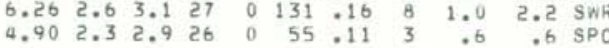




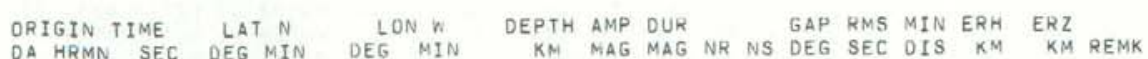

976 MAY 9 614 $43.371921 .55 \quad 155 \quad 7.95$ 15521.67 $\begin{array}{lllllll}10 & 827 & 4.05 & 19 & 23.27 & 155 & 4.44\end{array}$ $10 \quad 935 \quad 5.641923 .79 \quad 155 \quad 2.76$ $\begin{array}{lllllll}11 & 822 & 40.20 & 19 & 18.15 & 155 & 15.68\end{array}$ $11919 \quad 43.50 \quad 1920.39 \quad 155 \quad 7.10$ $12931 \quad 3.08$ 19 24.05 i5s 28.08 1211621.181924 .16 $12196 \quad 51.79 \quad 19 \quad 19.72 \quad 155 \quad 11.90$

$\begin{array}{lllllll}12 & 2241 & 17.13 & 19 & 23.27 & 155 & 17.15\end{array}$

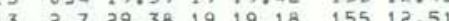

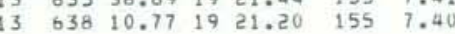
$\begin{array}{lllllll}13 & 919 & 21.08 & 19 & 21.31 & 155 & 3.32\end{array}$

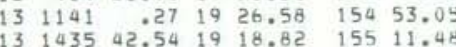

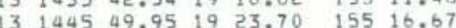
$132313 \quad 46.61 \quad 19 \quad 17.66 \quad 15521.40$

$13231532.501917 .21 \quad 15521.25$

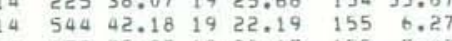

$\begin{array}{rrrrrrr}14 & 652 & 35.93 & 19 & 20.15 & 155 & 5.03 \\ 14 & 815 & 43.80 & 19 & 22.72 & 154 & 59.26\end{array}$

$14 \quad 912 \quad 42.17 \quad 19 \quad 17.38 \quad 155 \quad 30.51$ $14 \quad 1950 \quad 4.15 \quad 19 \quad 19.99 \quad 155 \quad 9.10$ $15 \quad 45426.32 \quad 19 \quad 19.62 \quad 15511.49$ $15740 \quad 51.48 \quad 1919.91$ is5 12.13

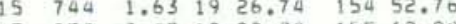
$\begin{array}{lllllll}15 & 850 & 2.45 & 19 & 22.3 \mathrm{~A} & 155 & 12.9 \mathrm{~A}\end{array}$ $1514 \quad 919.991922 .99 \quad 155 \quad 30.20$ 15 1444 23.761919 .59 155 11.00

$\begin{array}{rrrrrrr}15 & 1910 & 31.63 & 19 & 21.99 & 155 & 6.68 \\ 15 & 2139 & 58.45 & 19 & 25.77 & 155 & 16.76\end{array}$ $152150 \quad 24.66 \quad 1923.83 \quad 155 \quad 16.8$ $16 \quad 32419.341924 .19 \quad 15516.06$

$\begin{array}{llllllll}16 & 4 & 7 & 9.40 & 19 & 27.24 & 155 & 14.62\end{array}$ $16 \quad 436 \quad 33.24 \quad 1924.15 \quad 155 \quad 15.79$ $10 \quad 926 \quad 34.901927 .69 \quad 15514.13$ 16 15 $629.401924 .03 \quad 15516.10$

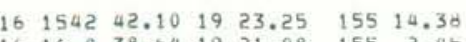
$16 \quad 1915 \quad 34.97 \quad 1920.16 \quad 155 \quad 11.7$ $\begin{array}{llllllllllll}9.72 & 3.4 & 3.6 & 30 & 0 & 71 & .08 & 9 & .5 & .3 & \text { UER }\end{array}$

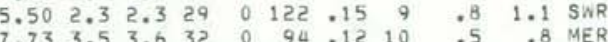

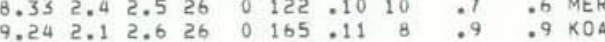
$\begin{array}{lllllllllll}9.47 & 2.9 & 3.1 & 26 & 0 & 98 & .08 & 7 & .6 & .4 & \text { UER }\end{array}$

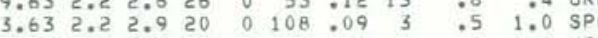

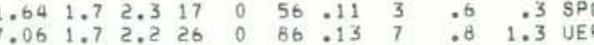

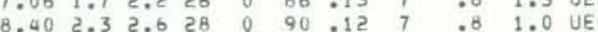

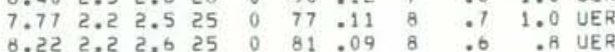
$\begin{array}{lllllllllllllll}7.03 & 2.7 & 3.1 & 25 & 0 & 114 & .10 & 11 & .0 & .7 & \text { MER }\end{array}$

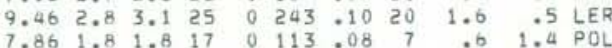
$\begin{array}{llllllllllll}1.74 & 2.2 & 3.0 & 15 & 0 & 77.09 & 3 & .5 & .3 & \mathrm{SPt}\end{array}$ $\begin{array}{lllllllllll}5.78 & 1.9 & 2.5 & 21 & 0 & 128 & .16 & 8 & 1.0 & 2.7 & \text { SWR }\end{array}$ $\begin{array}{lllllllllll}6.67 & 1.7 & 2.1 & 19 & 0 & 133 & .12 & 9 & 1.0 & 1.9 & \text { SWR }\end{array}$

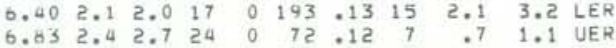

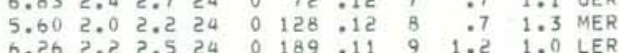
$\begin{array}{lllllllllll}9.56 & 3.1 & 3.2 & 28 & 0 & 100 & .14 & 13 & .9 & .6 & \text { HEA }\end{array}$

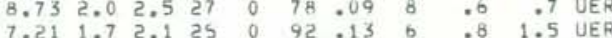

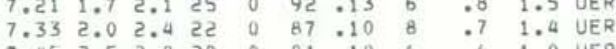
$\begin{array}{llllllllll}7.45 & 2.5 & 2.8 & 28 & 0 & 81.10 & 6 & .6 & 1.0 & \text { UER }\end{array}$

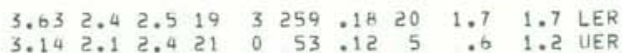

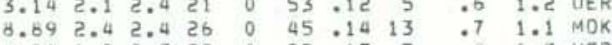

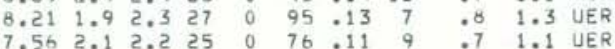
$\begin{array}{lllllllllll}7.52 & 2.2 & 2.6 & 21 & 0 & 74 & .10 & 0 & .6 & 1.0 & \text { UER } \\ 4.02 & 2.6 & 3.3 & 31 & 0 & 67 & .18 & 5 & .7 & 1.5 & \text { SPC }\end{array}$ $\begin{array}{lllllllllll}1.19 & 2.0 & 2.7 & 18 & 0 & 71 & .12 & 3 & .6 & .4 & \text { SPC }\end{array}$

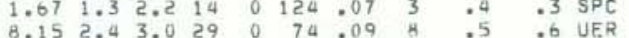

$\begin{array}{lllllllllll}31.65 & 2.3 & 2.4 & 35 & 0 & 52 & .11 & 8 & .8 & 1.8 & \text { DEP }\end{array}$

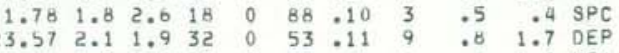
$\begin{array}{rrrrrrrrrrr}7.86 & 1.5 & 2.0 & 27 & 0 & 87 & .11 & 6 & .6 & 1.0 & \text { KOA } \\ 1.81 & 1.7 & 2.5 & 13 & 0 & 133 & .08 & 3 & .7 & .4 & \text { SPC }\end{array}$

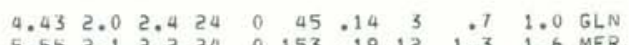

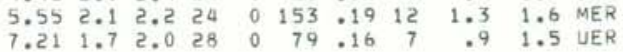

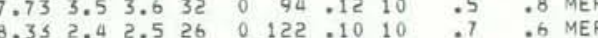

ORIGIN TIME
DA HRMN SEC N N LON W
DEG DIN DEG MIN YEAR MON DA MRMN SEC DEG MIN

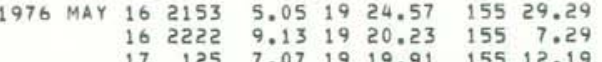
$\begin{array}{lllllll}17 & 125 & 7.07 & 19 & 19.91 & 155 & 12.19\end{array}$ $17145 \quad 6.091919 .78$ 155 16.26

$\begin{array}{lllllll}17 & 627 & 30.37 & 19 & 22.69 & 155 & 6.25\end{array}$ $\begin{array}{lllllll}17 & 847 & 17.06 & 19 & 27.52 & 154 & 54.25\end{array}$

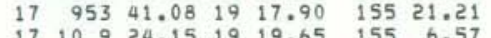

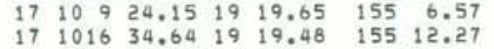
$\begin{array}{lllllll}17 & 1759 & 16.67 & 19 & 23.76 & 155 & 16.68\end{array}$

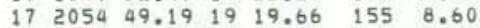
$172136 \quad 18.601920 .42 \quad 155 \quad 4.64$

18. 52058.1619 20.16 15550.97

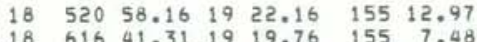
$1875 \quad 6.62$ 19 $9.08 \quad 15524.41$ $\begin{array}{lllllll}18 & 1044 & 56.03 & 19 & 19.39 & 155 & 11.43 \\ 18 & 1348 & 50.13 & 19 & 21.84 & 155 & 17.85\end{array}$ $\begin{array}{llllll}18 & 1410 & 58.49 \quad 19 & 19.45 \quad 155 \quad 15.87\end{array}$ 18 $145520.13 \quad 1924.04 \quad 155 \quad 26.93$

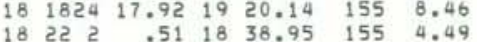
$\begin{array}{lllllll}18 & 2244 & 35.55 & 19 & 19.97 & 154 & 58.58\end{array}$

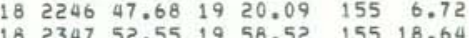
$19 \quad 132 \quad 20.991926 .26 \quad 155 \quad 29.92$ $19 \quad 40 \quad 11.21 \quad 1927.20 \quad 15529.29$

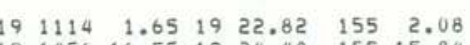

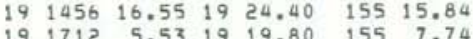
$19.1727 \quad 4.531919 .80 \quad 15512.09$

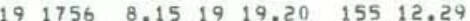

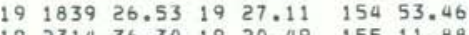
19231436.301920 .4915511 .88

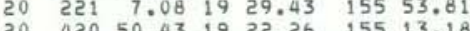
$20 \quad 542 \quad 41.62 \quad 1923.17 \quad 155 \quad 14.48$ $\begin{array}{llllllll}20 & 6 & 2 & 54.81 & 19 & 24.46 & 155 & 26.62\end{array}$ $\begin{array}{lllllll}20 & 631 & 25.44 & 19 & 23.20 & 155 & 14.94\end{array}$

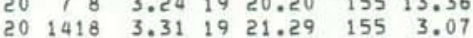

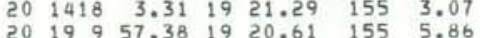

$\begin{array}{lllllll}20 & 1917 & 42.81 & 19 & 21.67 & 155 & 4.98\end{array}$

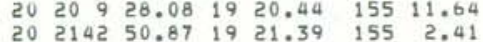

GAPTH AMP DUR RAP RMS MIN ERH ERT KM MAG MAG NR NS DEG SEC DIS KM KM REMK

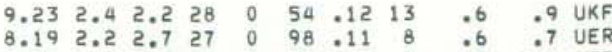

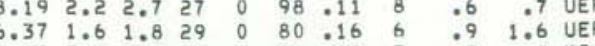

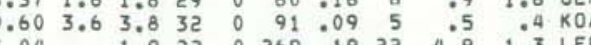

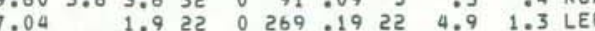
$\begin{array}{lllllllllll}8.12 & 2.6 & 3.0 & 29 & 0 & 68 & .12 & 8 & .6 & .8 & \text { UER }\end{array}$

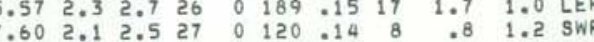

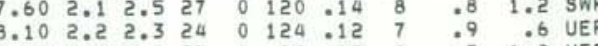

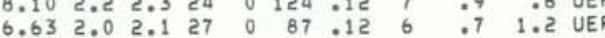
$\begin{array}{llllllllll}6.86 & 1.8 & 2.8 & 13 & 0 & 65 & .19 & 3 & 1.6 & 2.0 \\ \text { LPC }\end{array}$ $\begin{array}{llllll} & \end{array}$

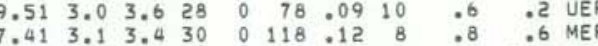
\begin{tabular}{rrrrrrrrrr}
7.41 & 3.1 & 3.4 & 30 & 0 & 118 & .12 & 8 & .8 & .6 \\
7.78 & 2.2 & 2.5 & 30 & 0 & 66 & .15 & 7 & .8 & 1.2 \\
\hline
\end{tabular} $\begin{array}{lllllllll}6.96 & 2.5 & 3.2 & 29 & 0 & 55.09 & 5 & .4 & .8 \\ 0 & \text { UER }\end{array}$ $\begin{array}{llllllllll}8.63 & 3.5 & 4.0 & 32 & 0 & 102 & .12 & 8 & .8 & .6 \\ 3 & .6 & \text { UER }\end{array}$ $\begin{array}{llllllllll}1.1 & 17 & 0 & 225 & .09 & 21 & 3.9 & 6.9 & \text { LER }\end{array}$ $\begin{array}{lllllllllll}5.96 & 2.0 & 2.2 & 28 & 0 & 97 & .15 & 7 & .9 & 1.8 & \text { UER } \\ 4.01 & 2.0 & 1.8 & 28 & 0 & 58 & .08 & 4 & .7 & 1.2 & \text { DEP }\end{array}$ $\begin{array}{lllllllllll}9.91 & 2.4 & 2.8 & 30 & 0 & 91 & .10 & 6 & .6 & .3 & \mathrm{KOA}\end{array}$

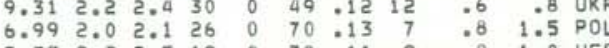
$\begin{array}{llllllllllll}7.33 & 2.2 & 2.5 & 19 & 0 & 78 & .11 & 9 & .8 & 1.8 & \text { UER } \\ 8.60 & & 1.6 & 25 & 2 & 289 & .13 & 72 & 4.1 & 20.3 & \text { PPL }\end{array}$

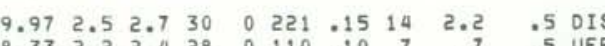

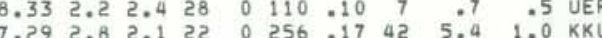

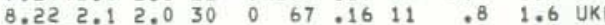
$9.30 \quad 2.5 \quad 2.6 \quad 32 \quad 0 \quad 60 \quad .15 \quad 12 \quad .7 \quad 1.3$ UKF $\begin{array}{llllllllllll}7.34 & 2.5 & 3.0 & 29 & 0 & 142 & .13 & 11 & .9 & .8 & \text { MER }\end{array}$

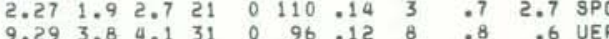

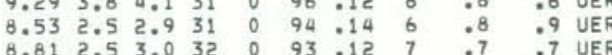
$\begin{array}{lllllllllll}8.63 & 2.6 & 2.8 & 27 & 0 & 229 & .11 & 19 & 1.6 & .5 & .5 \\ 7.0 R\end{array}$

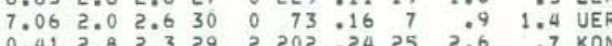

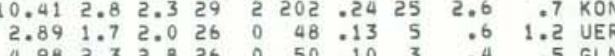
$\begin{array}{lllllll}4.98 & 2.3 & 2.8 & 26 & 0 & 50\end{array}$ $\begin{array}{llllllllllll}8.49 & 1.8 & 1.9 & 28 & 0 & 50 & .14 & 11 & .7 & 2.0 & \text { UKF } & 1.0\end{array}$

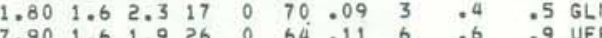

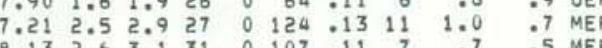
$\begin{array}{llllllllll}5.52 & 2.1 & 26 & 0 & 81 & .16 & 9 & .9 & 1.6 & \text { MER }\end{array}$ $\begin{array}{rrrrrrrrrrr}8.99 & 2.2 & 2.6 & 31 & 0 & 76 & .16 & 7 & .8 & .9 & .9 \\ 6.34 & 2.1 & 2.5 & 30 & 0 & 149 & .17 & 12 & 1.0 & 1.2 & \text { MER }\end{array}$ 


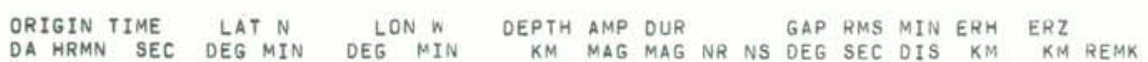
YEAR MON DA HRMN SEC DEG MIN DEG MIN 1976 MAY $202313 \quad 38.38 \quad 1920.68 \quad 155 \quad 6.73$ $21 \quad 75733.021919 .53 \quad 15512.35$ $21 \quad 932 \quad 40.611918 .18 \quad 155 \quad 16.62$ $21143948.981921 .19 \quad 15548.03$ $21 \quad 1722 \quad 20.28 \quad 1920.66 \quad 155 \quad 9.09$ $21 \quad 1953 \quad 9.83 \quad 1921.08 \quad 155 \quad 6.06$

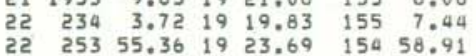
$\begin{array}{llllll}22 & 15 & 5 & 17.18 & 19 & 20.67 \quad 155 \quad 9.17\end{array}$

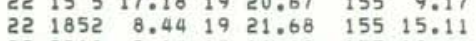

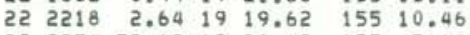

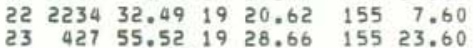

$\begin{array}{lllllll}23 & 449 & 33.07 & 19 & 20.42 & 155 & 4.25\end{array}$

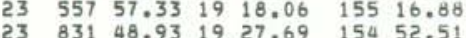
$23131113.83 \quad 1922.06 \quad 155 \quad 6.12$

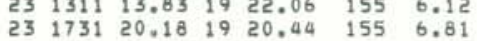

$\begin{array}{r}23173459.581920 .79 \quad 15511.62 \\ \hline \quad 2318325.751920 .49 \\ \hline\end{array}$

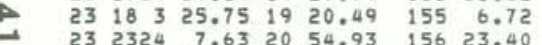
$\begin{array}{rrrrrrr}23 & 2324 & 7.63 & 20 & 54.93 & 156 & 23.40 \\ 24 & 121 & 50.68 & 19 & 21.00 & 155 & 13.47\end{array}$

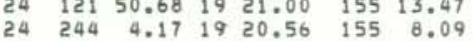
$24 \quad 546 \quad 55.74 \quad 19 \quad 21.12 \quad 155 \quad 13.49$

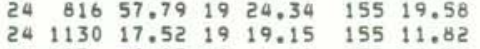
24123449.03 19 $6.27 \quad 15528.08$ $24 \quad 1641 \quad 12 \quad 1924.02 \quad 155 \quad 15.79$

$\begin{array}{llllllr}25 & 531 & 34.45 & 19 & 21.53 & 155 & 7.44 \\ 25 & 731 & 17.19 & 19 & 21.69 & 155 & 17.10\end{array}$

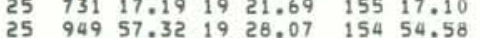
2594957.321928 .07 154 54.58

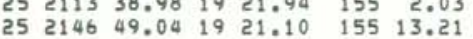

$\begin{array}{lllllllll}26 & 2 & 6 & 9.27 & 19 & 24.07 & 155 & 15.88\end{array}$

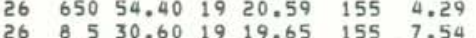

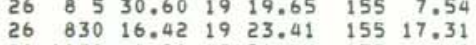

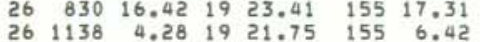

$\begin{array}{lllllll}26 & 1312 & 49.61 & 19 & 21.82 & 155 & 6.30 \\ 26 & 1413 & 58.67 & 19 & 19.27 & 155 & 13.93\end{array}$ $\begin{array}{lllllll}26 & 1413 & 58.67 & 19 & 19.27 & 155 & 13.93 \\ 26 & 16 & 34.91 & 19 & 19.83 & 155 & 11.02\end{array}$ $26 \quad 1738 \quad 12.451919 .61 \quad 155 \quad 8.16$

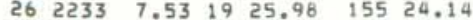

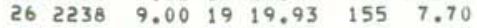

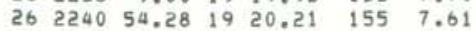

$\begin{array}{lllllllllll}8.32 & 2.2 & 2.6 & 28 & 0 & 97 & .10 & 7 & .5 & .6 & .6\end{array}$

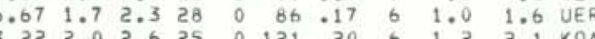

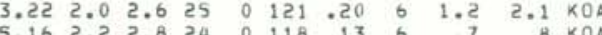

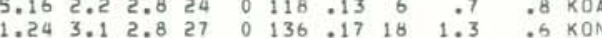

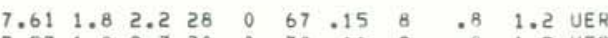
$\begin{array}{llllllllll}7.57 & 1.8 & 2.3 & 30 & 0 & 70 & .16 & 9 & .8 & 1.2 \\ 9 & .25 U^{2}\end{array}$ $\begin{array}{llllllllll}9.27 & 3.2 & 3.5 & 31 & 0 & 94 & .11 & 7 & .8 & \text {.5 UER }\end{array}$

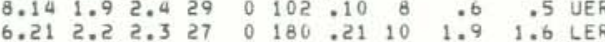
$\begin{array}{lllllllllll}8.03 & 1.8 & 2.1 & 27 & 0 & 67 & .14 & 8 & .9 & 1.0 & \text { UER }\end{array}$ $\begin{array}{lllllllllll}0.51 & 3.7 & 4.0 & 31 & 0 & 60 & 09 & 4 & .5 & .2 & K 0 A\end{array}$

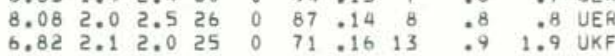
$\begin{array}{lllllllllllll}7.73 & 2.0 & 2.6 & 31 & 0 & 116 & .11 & 9 & .9 & .6 & \text { MER }\end{array}$

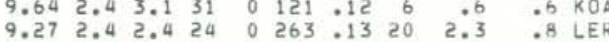
$6.091 .92 .628 \quad 0 \quad 75.1577 \quad .9 \quad 1.7$ UER \begin{tabular}{lllllllllll}
9.38 & 4.0 & 4.0 & 31 & 0 & 101 & .11 & 7 & .8 & .8 & .5 \\
\hline
\end{tabular}

$\begin{array}{llllllllll}7.74 & 2.6 & 2.7 & 22 & 0 & 152 & .18 & 8 & 1.4 & 1.3 \\ 7 & \text { UER }\end{array}$

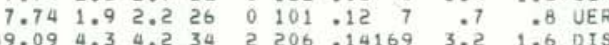

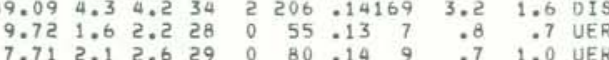
\begin{tabular}{llllllllll}
9.67 & 2.0 & 2.4 & 29 & 0 & 54 & .13 & 7 & .7 & .5 \\
\hline
\end{tabular}

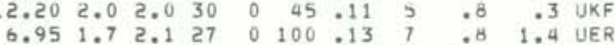

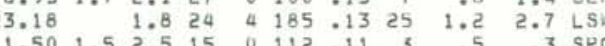
$\begin{array}{lllllllllll}8.14 & 2.6 & 3.0 & 30 & 0 & 76 & .12 & 8 & .6 & .7 & \text { UER }\end{array}$

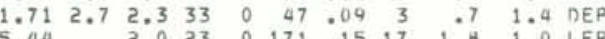

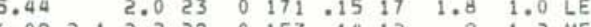

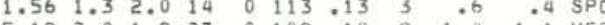

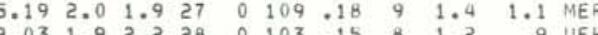

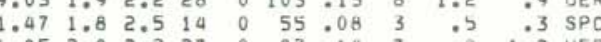

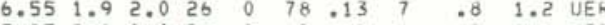

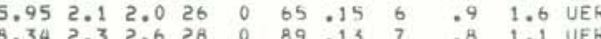

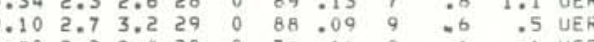
(1) $\begin{array}{lllllllllllll}9.11 & 3.1 & 3.2 & 31 & 0 & 66 & .13 & 9 & .6 & 1.1 & \text { UKF } \\ 7.59 & 1.9 & 2.4 & 28 & 0 & 95 & 13 & 8 & .7 & .7 & \text { UEE }\end{array}$

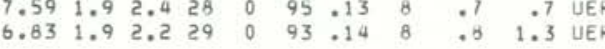
$8.70 \quad 1.623 .127 \quad 0 \quad 57.14 \quad 6 \quad 8 \quad 1.20$ UER
ORIGIN TIME LATN LON N YEAR MON DA HRMN SEC DEG MIN DEG MIN

$\begin{array}{lllllll}1976 & \text { MAY } 26 \quad 2347 \quad 5.78 & 19 & 17.33 & 155 & 15.82\end{array}$

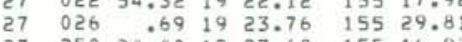
$\begin{array}{lllllll}27 & 250 & 24.48 & 19 & 23.68 & 155 & 16.92 \\ 27 & 337 & 33.64 & 19 & 20.19 & 155 & 7.21\end{array}$ $\begin{array}{lllllll}27 & 1349 & 9.15 & 19 & 19.23 & 155 & 11.60\end{array}$ $\begin{array}{lrrrrrr}27 & 1921 & 31.12 & 19 & 27.28 & 154 & 53.30 \\ 27 & 1951 & 9.49 & 19 & 27.45 & 154 & 55.91\end{array}$ $\begin{array}{llllllll}27 & 23 & 7 & 53.47 & 19 & 19.57 & 155 & 12.33\end{array}$ $\begin{array}{lllllll}28 & 050 & 32.59 & 19 & 16.97 & 155 & 22.7\end{array}$ $\begin{array}{lllllll}28 & 719 & 23.27 & 19 & 24.04 & 155 & 15.67\end{array}$ $\begin{array}{llllllll}28 & 12 & 8 & 45.51 & 19 & 23.03 & 155 & 4.6\end{array}$ $\begin{array}{lllllllll}28 & 14 & 1 & 19.91 & 19 & 22.10 & 155 & 6.72\end{array}$ $28145532.921920 .39 \quad 155 \quad 7.90$ $28181219.281920 .09 \quad 155 \quad 8.17$ $\begin{array}{lllllll}28 & 1958 & 46.27 & 19 & 21.67 & 155 & 3.11\end{array}$ $\begin{array}{lllllll}28 & 2320 & 36.62 & 19 & 23.27 & 155 & 17.00\end{array}$ $\begin{array}{lllllll}29 & 129 & 29.39 & 19 & 22.76 & 155 & 4.85\end{array}$ 29 1220 55.60 19 23.63 1556.31 $29 \begin{array}{llllll}1714 & 42.83 & 19 & 22.12 & 155 & 13.21\end{array}$ $\begin{array}{lllllll}29 & 1727 & 25.32 & 19 & 19.94 & 155 & 12.09\end{array}$ $\begin{array}{lllllll}29 & 1858 & 6.45 & 19 & 19.42 & 155 & 12.45\end{array}$ 29195051.1619 24.89 15516.12 $30 \quad 1010 \quad 9.71 \quad 1923.21 \quad 15514.60$

$\begin{array}{llllllll}30 & 11 & 2 & 26.73 & 19 & 22.14 & 155 & 13.14\end{array}$ $\begin{array}{lllllll}30 & 1131 & 14.78 & 19 & 21.91 & 155 & 12.92 \\ 30 & 1229 & 9.53 & 19 & 23.32 & 155 & 14.97\end{array}$ 300163232.58 19 20.23 $155 \quad 6.30$ 30173232.581920 .23 155 0.30

$\begin{array}{lllllll}30 & 1743 \quad 16.73 & 19 & 18.85 & 155 & 15.05\end{array}$ $\begin{array}{lllllll}30 & 197 & 53.92 & 19 & 22.76 & 155 & 24.66 \\ 30 & 1944 & 28.24 & 19 & 23.42 & 155 & 14.75\end{array}$ $300202558.31 \quad 19 \quad 19.76 \quad 155 \quad 11.92$ $\begin{array}{lllllll}30 & 2022 & 58.31 & 19 & 19.76 & 155 & 11.92\end{array}$

$\begin{array}{lllllll}31 & 146 & 3.02 & 19 & 27.38 & 154 & 52.62\end{array}$

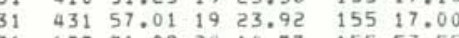
31 6टा ट11.09 टर 14.077 155 53.5

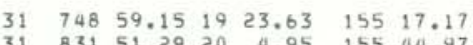

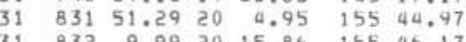
$\begin{array}{lllllll}31 & 832 & 9.99 & 20 & 15.86 & 155 & 46.17\end{array}$
DEPTH AMP DUR GAP RMS MIN ERH ERZ MAG MAG NR NS DEG SEC DIS KH KM REMMK $\begin{array}{lllllllllll}9.75 & 2.1 & 2.6 & 30 & 0 & 133 & .13 & 6 & .9 & .3 & \text { KOA }\end{array}$

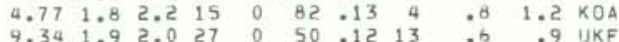

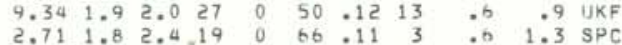

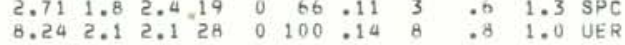

$\begin{array}{llllllllll}6.87 & 1.9 & 2.0 & 26 & 0 & 100 & .12 & 7 & .7 & 1.2\end{array}$ $\begin{array}{lllllllllll}9.93 & 2.9 & 3.1 & 32 & 0 & 59 & .14 & 13 & .7 & .4 & \text { MOK }\end{array}$

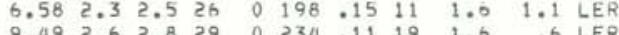

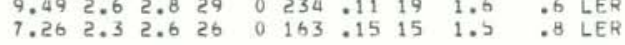

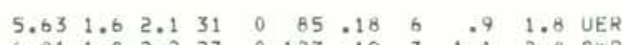

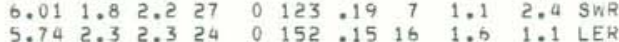

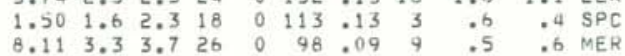
\begin{tabular}{lllllllllll}
9.25 & 2.4 & 2.6 & 24 & 0 & 72 & .11 & 8 & .8 & .5 & .5 \\
\hline & UER
\end{tabular}

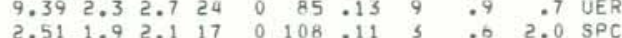

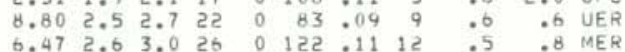

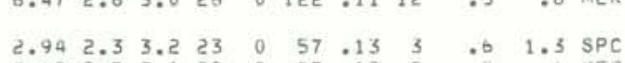

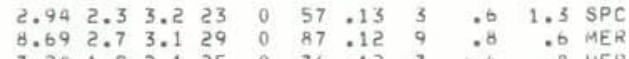

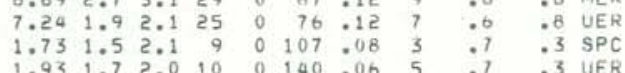
$\begin{array}{lllllllllll}1.93 & 1.7 & 2.0 & 10 & 0 & 140 & .06 & 5 & .7 & .3 & .3 \\ \text { UER }\end{array}$ $\begin{array}{lllllllllll}7.27 & 2.1 & 2.4 & 15 & 0 & 190 & .08 & 8 & 1.0 & 2.0 & \text { UER }\end{array}$

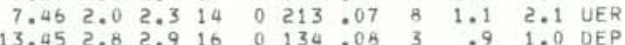

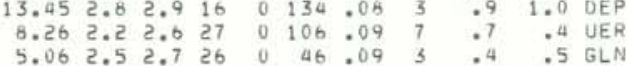
$\begin{array}{lllllllllll}5.89 & 2.3 & 2.9 & 24 & 0 & 72 & .11 & 6 & .0 & 1.2 & \text { UER } \\ 3.04 & 1.7 & 1.8 & 22 & 0 & 59 & .09 & 5 & .4 & 2.3 & \text { UER }\end{array}$

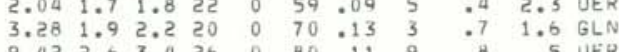

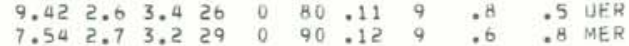

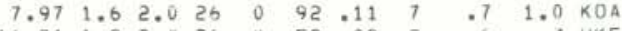

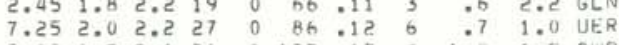

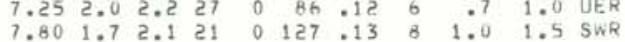

$\begin{array}{lllllllllll}8.78 & 2.4 & 2.6 & 24 & 0 & 263 & .16 & 21 & 4.9 & 1.0 & \text { LER } \\ 3.67 & 3.0 & 2.8 & 24 & 0 & 56 & .15 & 3 & .7 & 1.5 & \text { SPC }\end{array}$

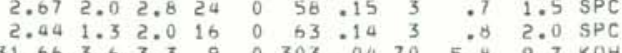

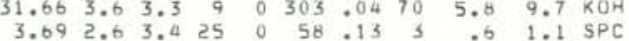
$\begin{array}{llllllllllll}1.88 & 1.4 & 2.3 & 15 & 0 & 55.13 & 3 & .7 & .4 & \mathrm{SPC}\end{array}$ $\begin{array}{llllllllllll}2.68 & 4.1 & 3.9 & 6 & 2 & 175 & .07 & 75 & 10.1 & 5.6 & 015\end{array}$ 
HVO EARTHQUAKE SUMMARY LIST

PAGE 25

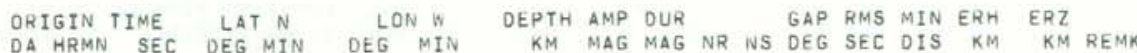

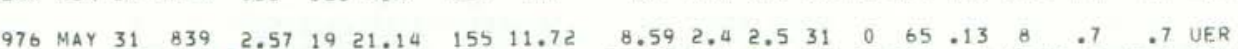

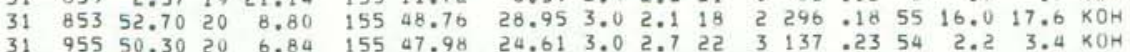

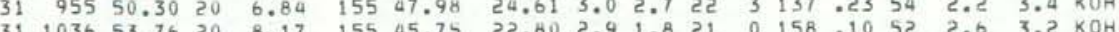

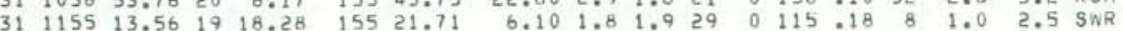

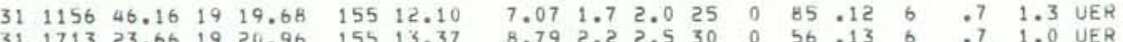

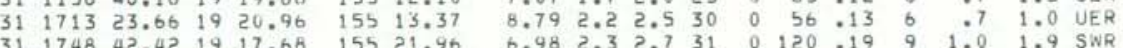

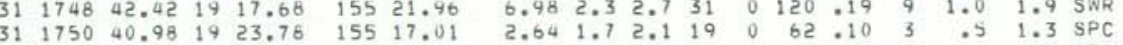

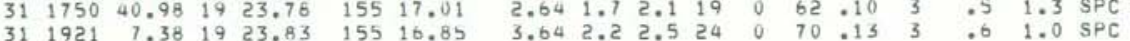

JUN $1 \quad 5 \quad 8 \quad 35.86 \quad 19 \quad 19.96 \quad 155 \quad 8.48$

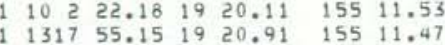
$134315.001927 .56 \quad 15451.91$

$1628 \quad 53.28 \quad 19 \quad 19.38 \quad 155 \quad 19,19$

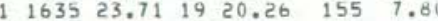

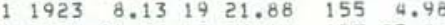

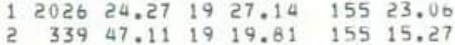

$\begin{array}{lllllll}2 & 353 & 38.16 & 19 & 22.14 & 155 \quad .28\end{array}$ द $851 \quad 36.92 \quad 1925.97 \quad 15455.43$

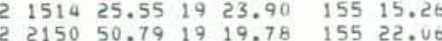

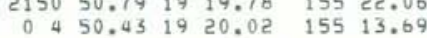

$\begin{array}{llllllll}3 & 2 & 1 & 11.72 & 19 & 20.06 \quad 155 \quad 3.97\end{array}$ $\begin{array}{llllllll}3 & 7 & 6 & 50.18 & 19 & 19.67 & 155 & 11.76\end{array}$ $\begin{array}{llllll}922 & 35.52 & 19 & 20.98 & 155 & 10.75\end{array}$

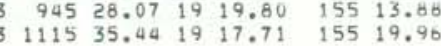

$\begin{array}{lllllll}3 & 1525 & 42.50 & 19 & 20.24 & 155 & 7.27\end{array}$ $32038 \quad 53.35$ 19 $26.76 \quad 155 \quad 25.32$

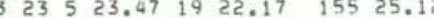
4
4 $\quad 3 \quad 22.721921 .38 \quad 155 \quad 13.41$

$4 \begin{aligned} & 446 \quad 13.06 \quad 1922.00 \quad 155 \quad 1.12\end{aligned}$

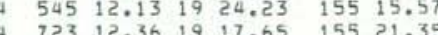
4
4 103110.64 19 19.37 1 15512.09

$4 \begin{array}{lllllll}4427 & 46.90 & 19 & 15.49 & 155 & 24.96\end{array}$

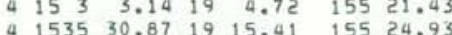

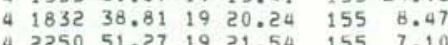

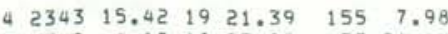

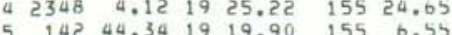

HVO EARTHQUAKE SUMMARY LIST

PAGE 26

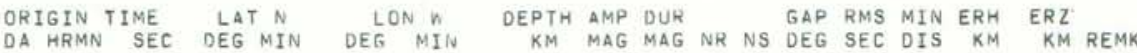

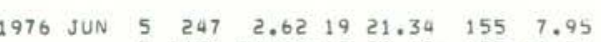
$\begin{array}{lllllll}5 & 1056 & 54.18 & 19 & 20.12 & 155 & 11.51 \\ 5 & 1118 & 20.99 & 19 & 21.59 & 155 & 7.40\end{array}$ $51344 \quad 44.201925 .52 \quad 155 \quad 16.52$ $\begin{array}{llllllll}5 & 14 & 4 & 2.03 & 19 & 25.51 & 155 & 16.43\end{array}$ $\begin{array}{llllllll}5 & 17 & 0 & 54.97 & 19 & 17.89 & 155 & 16.87 \\ 5 & 1752 & 22.73 & 19 & 19.70 & 155 & 11.79\end{array}$ $\begin{array}{rrrrrrr}5 & 1752 & 22.73 & 19 & 19.70 & 155 & 11.79 \\ 5 & 2046 & 3.20 & 19 & 3.73 & 155 & 27.66\end{array}$

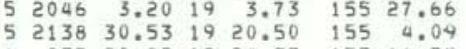
$037 \quad 20.02 \quad 1924.33 \quad 155 \quad 14.75$

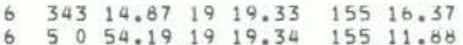

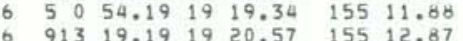

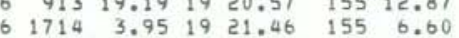

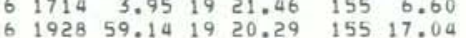

$\begin{array}{lllllll}7 & 719 & 47.08 & 19 & 18.52 & 155 & 13.32\end{array}$ $\begin{array}{lllllll}7 & 1039 & 37.57 & 19 & 19.15 & 155 & 15.64\end{array}$ $\begin{array}{lllllll}7 & 1126 & 10.28 & 19 & 24.06 & 155 & 15.97 \\ 7 & 2120 & 37.03 & 19 & 20.72 & 155 & 7.73\end{array}$

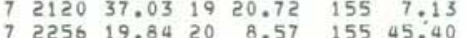

$8 \quad 210 \quad 10.84 \quad 1925.58 \quad 155 \quad 17.10$ $\begin{array}{llllllll}8 & 1413 & 49.47 & 19 & 21.76 & 155 & 3.30 \\ 8 & 1743 & 32.63 & 19 & 27.01 & 154 & 54.14\end{array}$ $\begin{array}{lllllll}8 & 1743 & 32.63 & 19 & 27.01 & 154 & 54.14 \\ B & 1949 & 02.96 & 19 & 21\end{array}$ $\begin{array}{lllllll}8 & 1949 & 92.96 & 19 & 21.94 & 155 & 7.01 \\ 8 & 2132 & 46.00 & 19 & 20.70 & 155 & 13.26\end{array}$

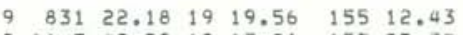

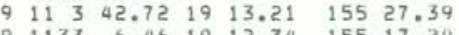

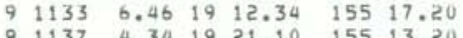
$\begin{array}{lllllll}9 & 1137 & 4.34 & 19 & 21.10 & 155 & 13.20 \\ 9 & 1439 & 43.64 & 19 & 19.74 & 155 & 7.54\end{array}$

$9 \begin{aligned} & 9827 \quad 51.14 \quad 19 \quad 27.85 \quad 154 \\ & 92.77\end{aligned}$ $92211 \quad 2.41 \quad 1920.60 \quad 155 \quad 11.25$ $\begin{array}{lllllll}10 & 015 & 3.85 & 19 & 20.07 & 155 & 7.32\end{array}$

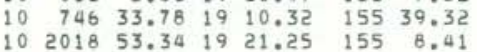

$\begin{array}{llllllll}10 & 22 & 5 & 5.23 & 19 & 19.78 & 155 & 8.51\end{array}$

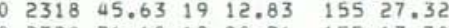
$\begin{array}{lllllll}10 & 2328 & 36.60 & 19 & 20.36 & 155 & 13.70\end{array}$

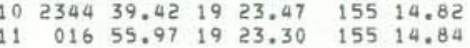

$\begin{array}{llllllll}11 & 3 & 5 & 35.62 & 19 & 47.43 & 155 & 25.05\end{array}$ $\begin{array}{lllllll}11 & 1437 & 49.53 & 18 & 55.52 & 155 & 17.60\end{array}$ $12 \quad 6 \quad 6 \quad 8.28 \quad 1920.76 \quad 155 \quad 6.64$

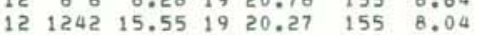
$\begin{array}{lllllll}12 & 1359 & 49.28 & 19 & 23.67 & 155 & 16.92\end{array}$ $\begin{array}{lllllll}12 & 14 & 31.31 & 19 & 23.65 & 155 & 16.92\end{array}$ $\begin{array}{lllllllllll}7.89 & 1.8 & 2.0 & 26 & 0 & 73 & .14 & 9 & .8 & 1.0 \\ .0 & .0 & \text { UER }\end{array}$

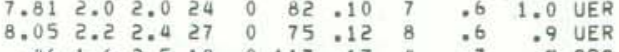
$\begin{array}{lllllllllll}9.01 & 1.7 & 2.2 & 31 & 0 & 124 & .13 & 6 & .7 & .9 & K O A\end{array}$

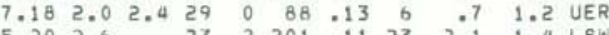

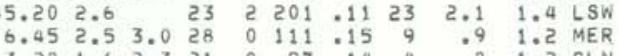
$\begin{array}{llllllllll}3.28 & 1.6 & 2.3 & 21 & 0 & 97.14 & 4 & .8 & 1.2 & \text { GLN }\end{array}$

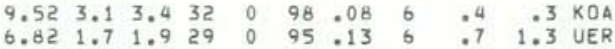

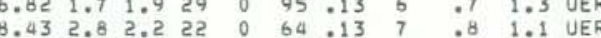
$\begin{array}{lllllllllll}7.60 & 2.2 & 2.4 & 31 & 0 & 83 & .12 & 7 & .6 & .8 & .8 \\ 3\end{array}$ $\begin{array}{lllllllllll}1.40 & 3.5 & 3.3 & 35 & 0 & 93 & .09 & 6 & .7 & 1.3 & \text { OEP }\end{array}$

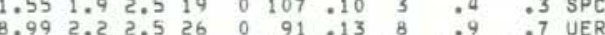

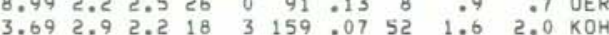
$\begin{array}{llllllllllll}.68 & 1.7 & 2.7 & 19 & 0 & 116 & .17 & 5 & .9 & .6 & \text { SPC }\end{array}$

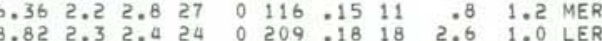

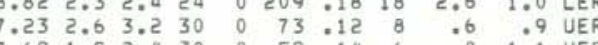
$\begin{array}{llllllllll}6.92 & 2.4 & 2.8 & 31 & 0 & 84 & .14 & 6 & .8 & 1.3 \\ \text { UER }\end{array}$

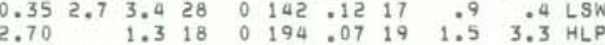

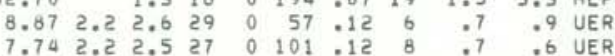
$\begin{array}{llllllllllll}8.81 & 2.8 & 3.2 & 31 & 0 & 74 & .10 & 8 & .6 & .7 & \text { UER }\end{array}$ $\begin{array}{lllllllllll}8.55 & 3.3 & 3.7 & 32 & 0 & 100 & .11 & 8 & .7 & .6 & .6 \\ 0 E R\end{array}$

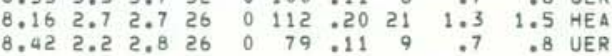
$\begin{array}{lllllllllll}9.55 & 3.2 & 3.7 & 33 & 0 & 79.09 & 10 & .6 & .2 & 0 & 0\end{array}$ $\begin{array}{llllllllll}9.73 & 2.7 & 3.0 & 22 & 0 & 145 & .16 & 18 & 1.3 & .5 \\ 18 & .5 & \text { LSW }\end{array}$ $\begin{array}{llllllllllll}10.23 & 2.4 & 2.9 & 30 & 0 & 57 & .10 & 6 & .6 & .3 & .3 & \text { UER }\end{array}$

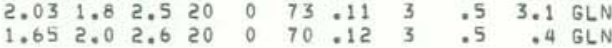
$\begin{array}{llllllllllll}8.00 & 2.6 & 2.5 & 33 & 0 & 81 & .10 & 22 & .7 & 1.9 & \mathrm{kKU}\end{array}$

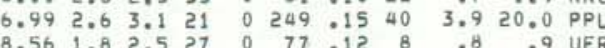
$\begin{array}{lllllllllll}9.12 & 3.1 & 3.4 & 25 & 0 & 84 & .07 & 9 & .5 & .4 & \end{array}$ $\begin{array}{llllllllllll}1.80 & 1.1 & 2.0 & 12 & 0 & 71 & .06 & 3 & .4 & .2 & \text { SPC }\end{array}$

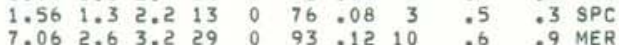

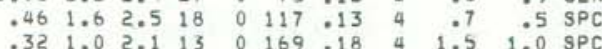
$\begin{array}{llllllllll}7.66 & 2.4 & 2.6 & 24 & 0 & 260 & .14 & 20 & 2.4 & 1.0\end{array}$

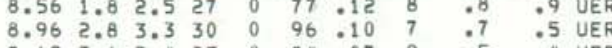


hVO EARTHQUAKE SUMMARY LIST

PAGE 27

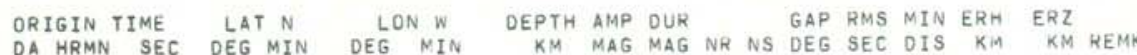

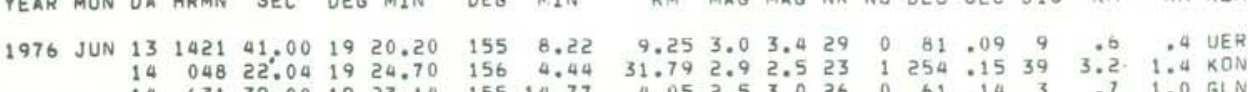

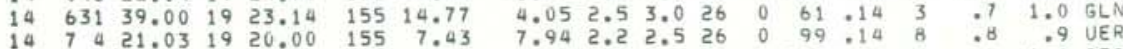

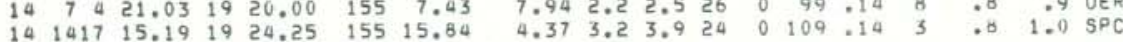
$\begin{array}{lllllll}14 & 1432 & 41.09 & 19 & 19.08 & 155 & 13.36\end{array}$ 1417034.341919 .97 155 8.25

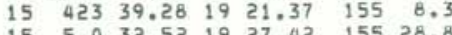
$15 \quad 315 \quad 31.95 \quad 19223.87 \quad 155 \quad 6.31$

$\begin{array}{lllllll}15 & 852 & 10.59 & 19 & 12.59 & 155 & 35.77\end{array}$

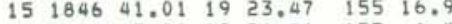
$16 \quad 91715.921921 .81 \quad 155 \quad 4.84$ $16174418.201923 .17 \quad 15514.62$

$\begin{array}{lllllll}16 & 2117 & 42.41 & 19 & 17.56 & 155 & 27.93\end{array}$

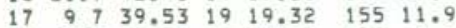

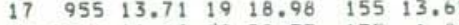

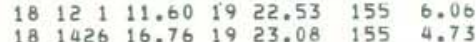

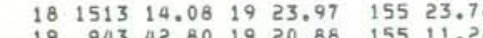
w $19154922.611919 .44 \quad 15512.45$

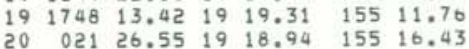

$\begin{array}{llllllll}20 & 025 & 11.27 & 19 & 23.50 & 155 & 15.0\end{array}$ $20 \quad 3 \quad 6 \quad 23.8119$

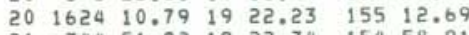
$\begin{array}{lllllll}21 & 344 & 51.92 & 19 & 22.34 & 154 & 58.91 \\ 21 & 453 & 37.14 & 19 & 54.73 & 155 & 33.63\end{array}$

$\begin{array}{llllll}21 & 513 & 56.13 \quad 19 & 24.13 \quad 155 \quad 16.15\end{array}$ $21915 \quad 18.16 \quad 19 \quad 24.15 \quad 155 \quad 15.3$ $\begin{array}{lllllll}21 & 952 & 3.60 & 19 & 22.91 & 155 & 14.06\end{array}$ $\begin{array}{lllllll}21 & 1427 & 49.74 & 19 & 22.00 & 155 & 13.04 \\ 21 & 1446 & 46.29 & 19 & 22.41 & 155 & 11.88\end{array}$

21 $1456 \quad 11.34 \quad 19 \quad 22.24 \quad 155 \quad 12.61$ 211539.68 19 22.09 15512.55 21 174257.461922 .2915512 .95

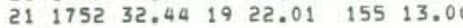

$21 \quad 1816 \quad 27.24 \quad 1922.15 \quad 155 \quad 12.92$ 21 183554.31 19 22.23 15513.13 21 $1859 \quad 45.86 \quad 1922.15 \quad 155 \quad 13.419$

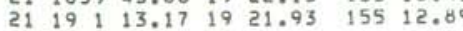

$\begin{array}{llllllll}21 & 19 & 3 & 34.10 & 19 & 22.27 & 155 & 13.1\end{array}$ ट1 19627.29 19 25.29 $\begin{array}{lllllllllll}9.28 & 2.8 & 3.2 & 25 & 0 & 74 & .12 & 7 & .0 & 1.0 & \text { UER }\end{array}$

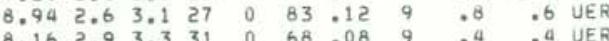

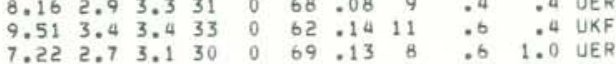

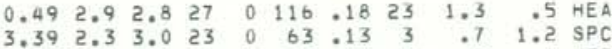

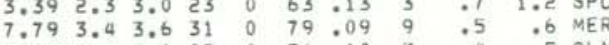

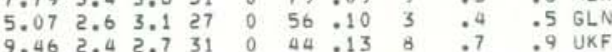
$\begin{array}{llllllllll}8.47 & 2.4 & 2.7 & 29 & 0 & 45 & .15 & 9 & .7 & 1.3 \\ 6 E A\end{array}$ $\begin{array}{lllllllllll}6.74 & 2.5 & 2.8 & 30 & 0 & 94 & .14 & 0 & .8 & 1.3 & \text { UER } \\ 6.96 & 2.5 & 3.0 & 20 & 0 & 68 & 1.16 & 7 & .9 & 1.5 & \text { POL }\end{array}$

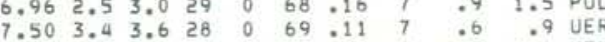

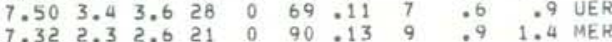

$\begin{array}{lllllllllll}8.39 & 2.3 & 2.8 & 30 & 0 & 45 & .13 & 7 & .6 & .9 & \text { UKF }\end{array}$

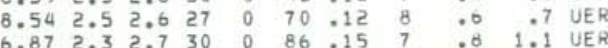

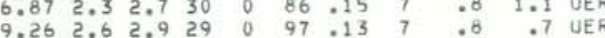
$\begin{array}{llllllllllll}9.26 & 2.6 & 2.9 & 29 & 0 & 97 & .13 & 7 & .8 & 97 & 952\end{array}$

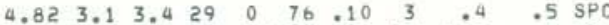
$\begin{array}{lllllllllll}0.64 & 3.1 & 3.5 & 23 & 0 & 132 & .17 & 22 & 1.3 & .6 & \text { LSW }\end{array}$

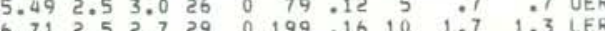

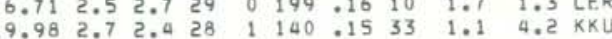

$\begin{array}{llllllllllll}1.62 & 2.0 & 2.6 & 13 & 0 & 109 & .16 & 3 & 1.0 & .6 & \mathrm{SPC}\end{array}$ $\begin{array}{llllllllll}.08 & 2.9 & 19 & 0 & 108 & 10 & 3 & .5 & 3.1 & \mathrm{SPC}\end{array}$

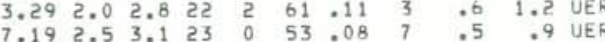

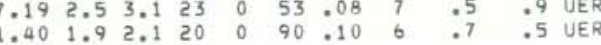
$3.432 .02 .222 \quad 0 \quad 60.1455 \quad .831 .4$ UER

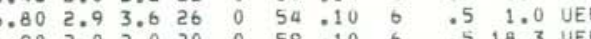

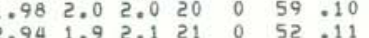
\begin{tabular}{lllllllll}
5.03 & 2.2 & 2.6 & 27 & 0 & 51.15 & 6 & .7 & .9 \\
\hline
\end{tabular} \begin{tabular}{llllllllll}
5.39 & 3.1 & 3.5 & 28 & 0 & 50.12 & 5 & .5 & .5 & .5 \\
\hline
\end{tabular}

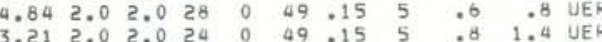
$\begin{array}{lllllllllll}6.57 & 2.7 & 2.7 & 27 & 0 & 49 & .09 & 6 & .5 & .8 & \text { UER } \\ 1.71 & 1.9 & 2.1 & 22 & 0 & 52 & .11 & 5 & .5 & .4 & 4\end{array}$ $\begin{array}{lllllllllll}0.15 & 3.4 & 3.7 & 27 & 0 & 54 & .09 & 6 & .4 & 1.0 & \text { UER }\end{array}$ प.4.
HVO EARTHQUAAEE SUMMAKY LIST

PAGE 28 ORIGIN TIME LATN LON N DEPTH AMP DUR GAP RMS MIN ERH ERZ YEAR MON DA HRMN SEC DEG MIN DEg MIN KM MAG MAG NR NS DEG SEC DTS KM KM REMK 1976 JUN $211927 \quad 7.43 \quad 1922.26 \quad 153 \quad 13.01$ $21 \quad 193513.32 \quad 1922.49 \quad 15513.34$ $21941 \quad 6.18 \quad 1922.17 \quad 155 \quad 15.05$ $\begin{array}{lllllll}21 & 1951 & 1.33 & 19 & 22.26 & 155 & 13.10\end{array}$

$\begin{array}{llllllll}21 & 20 & 0 & 34.66 & 19 & 22.34 & 155 & 13.25\end{array}$ $212012 \quad 17.12 \quad 1922.66 \quad 15514.04$ $212015 \quad 12.03 \quad 1922.81 \quad 155 \quad 5.04$ $21203508.041922 .63 \quad 15514.00$ $\begin{array}{lllllll}21 & 2038 & 50.23 & 19 & 20.25 & 155 & 13.47\end{array}$ $\begin{array}{lllllll}21 & 2118 & 37.95 & 19 & 22.40 & 155 & 12.85\end{array}$ $2127 \quad 28.991923 .17 \quad 155 \quad 14.04$ $212143 \quad 23.511923 .16 \quad 155 \quad 14.63$

$\begin{array}{lllllll}21 & 2148 & 12.93 & 19 & 21.35 & 155 & 12.44\end{array}$

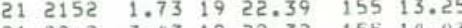
ट1 22 ट 3.431922 .32 155 13.03 ट1 ट2 $637.061923 .16 \quad 15510.57$

$\begin{array}{lllllll}21 & 2241 & 19.86 & 19 & 22.36 & 155 & 12.73\end{array}$ $\begin{array}{lllllll}21 & 2323 & 47.47 & 19 & 23.13 & 155 & 14.36\end{array}$ $\begin{array}{llll}21 & 2338 & 14.28 & 19 \\ 22.61 & 155 & 14.04\end{array}$ 21 235530.06 19 23.615215514 .043

$\begin{array}{lllllll}22 & 029 & 47.86 & 19 & 22.69 & 155 & 14.25\end{array}$ टट $03115.47 \quad 1922.44 \quad 15514.04$ 2203219.691922 .22 155 12.90 2203447.501922 .6115513 .98

$22 \quad 041 \quad 10.92 \quad 1922.54 \quad 155 \quad 14.12$ $2504740.901922 .81 \quad 15514.04$

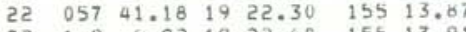
22 11 5.921922 .68 155 13.91

$22 \quad 2 \quad 0 \quad 39.89 \quad 19 \quad 23.17 \quad 155 \quad 14.74$

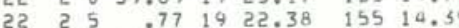
$\begin{array}{lllllll}22 & 224 & 59.79 & 19 & 22.57 & 155 & 14.23\end{array}$ 2523918.641922 .44 155 14.18

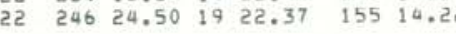

$\begin{array}{lllllll}22 & 247 & 42.04 & 19 & 22.97 & 155 & 14.35\end{array}$ $\begin{array}{lllllll}22 & 252 & 14.27 & 19 & 22.57 & 155 & 14.25\end{array}$ $22 \quad 257 \quad 5.091923 .23 \quad 15514.73$

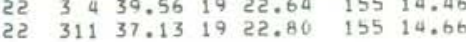

$22 \quad 341 \quad 9.921923 .13 \quad 15514.60$

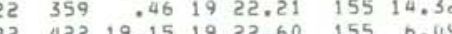

$\begin{array}{lllllllllll}5.90 & 2.0 & 2.3 & 25 & 0 & 49 & .15 & 5 & .7 & 1.2 & \text { UER }\end{array}$

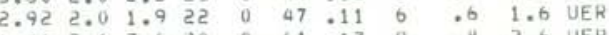

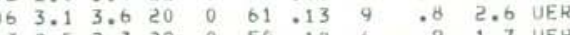

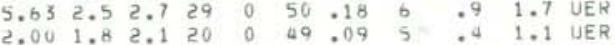
\begin{tabular}{lllllllllll}
5.30 & 3.3 & 3.9 & 26 & 0 & 63 & .13 & 7 & .6 & .7 & .7 \\
\hline
\end{tabular} $9.383 .23 .5 \quad 30 \quad 0$ की:

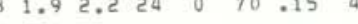

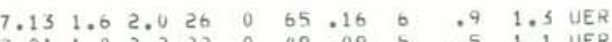

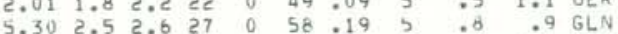
$\begin{array}{lllllllllll}3.42 & 3.0 & 3.4 & 20 & 0 & 64 & .13 & 3 & .7 & 1.4 & 6 L N \\ 4.52 & 2.0 & 2.5 & 26 & 0 & 46 & .13 & 3 & 0 & 1.1 & 6 L N\end{array}$ $\begin{array}{llllllllll}.53 & 1.9 & 2.4 & 19 & 0 & 60 & .19 & 0 & .8 & 4.4 \text { UER }\end{array}$ $\begin{array}{lllllllllll}5.62 & 2.4 & 2.8 & 23 & 0 & 73 & .08 & 6 & .5 & .9 & \text { UER } \\ 1.66 & .6 & 9 & 9 & 0 & 0\end{array}$

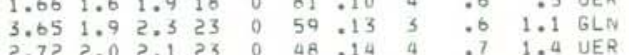

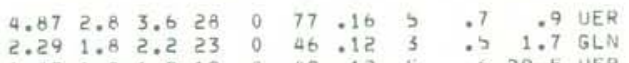

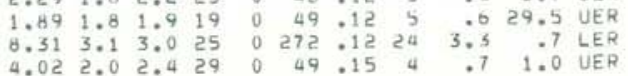
$\begin{array}{lllllllllll}3.45 & 2.1 & 2.5 & 27 & 0 & 56 & .15 & 4 & .7 & 1.2 & \text { UER } \\ 4.40 & 2.4 & 2.7 & 30 & 0 & 50 & .16 & 4 & .7 & 1.0 & \text { UER }\end{array}$

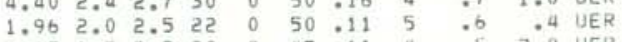

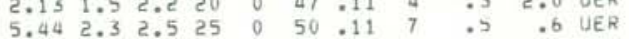
\begin{tabular}{llllllllll}
1.63 & 1.7 & 1.9 & 20 & 0 & 49 & .10 & 4 & .5 & .6 \\
\hline
\end{tabular}

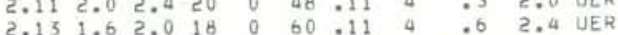

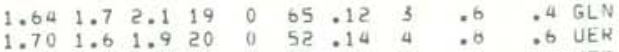

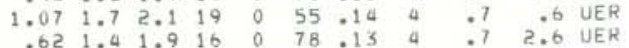

\begin{tabular}{lllllllllll}
.08 & 1.4 & 2.0 & 17 & 0 & 65 & .13 & 3 & .6 & .4 & .4 \\
\hline
\end{tabular}

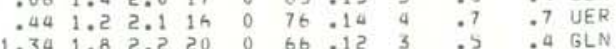

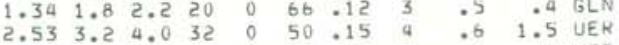

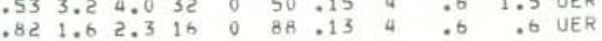

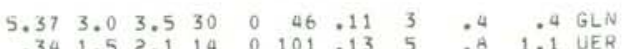

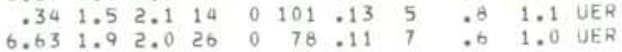
$\begin{array}{lllllllllll}14 & 2.1 & 2.1 & 23 & 0 & 4 H & .14 & 4 & .7 & 1.1 & \text { UER }\end{array}$

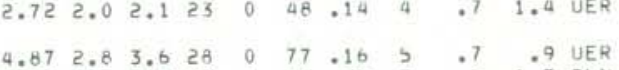

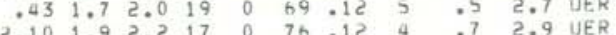

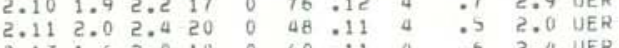

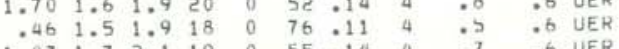


$\begin{array}{llllll}\text { ORIGIN TIME } & \text { LAT N } & \text { LON } N & \text { DEPTH AMP DUR N GAP RMS MIN ERH ERZ } \\ \text { OA HRMN SEC DEG MIN } & \text { DEG MIN } & \text { KM MAG MAG NR NS DEG SEC OIS KM KM REMK }\end{array}$ 1976 JUN $22 \quad 425 \quad 46.32 \quad 1922.34 \quad 155 \quad 14.13$ $\begin{array}{lllllll}22 & 433 & 38.46 & 19 & 19.99 & 155 & 7.00\end{array}$ 22 $53110.651923 .17 \quad 15514.30$

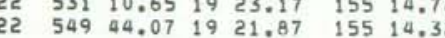

$\begin{array}{llllllll}22 & 6 & 1 & 44.11 & 19 & 23.27 & 155 & 14.7\end{array}$

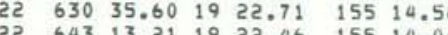
$22,646 \quad 41.041923 .16$ 155 14.52

$\begin{array}{lllllll}22 & 742 & 17.23 & 19 & 23.55 & 155 & 15.16\end{array}$ $\begin{array}{llllll}725 & 65.86 & 19 & 23.45 & 155 & 15.16\end{array}$ $\begin{array}{lllll} & \end{array}$

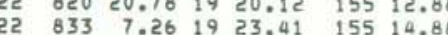

$\begin{array}{lllllll}22 & 1237 \quad 36.76 & 19 & 46.90 & 156 & 2.24\end{array}$

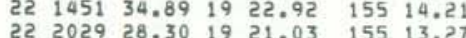
22 20ट9 $28.301921 .03 \quad 15513.27$ $22235354.3519-23.28$ 155 14.50

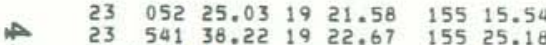
$\begin{array}{lllllll}23 & 1413 & 10.74 & 19 & 19.80 & 155 & 8.6\end{array}$

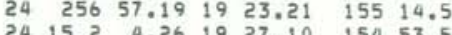

$\begin{array}{lllllll}24 & 1640 & 8.68 & 19 & 19.15 & 155 & 13.50\end{array}$ $24 \quad 1947 \quad 53.85 \quad 1920.31 \quad 155 \quad 11.90$ $24201727.31 \quad 1920.93 \quad 15516.73$

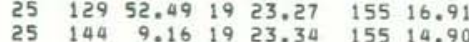

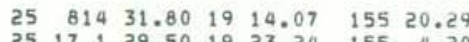

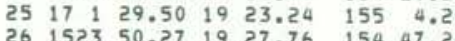
$\begin{array}{lllllll}26 & 1523 & 50.27 & 19 & 27.76 & 154 & 47.28 \\ 26 & 2248 & 37.68 & 19 & 19.44 & 155 & 16.02\end{array}$ $27 \quad 2318 \quad 7.02 \quad 1923.19 \quad 155 \quad 14.5$

$\begin{array}{lllllllll}28 & 8 & 6 & 54.39 & 21 & 1.41 & 156 & 3.54\end{array}$

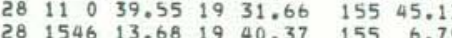

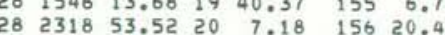

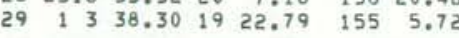
$\begin{array}{lllllll}29 & 1627 & 14.88 & 19 & 17.84 & 155 & 16.24\end{array}$ $30 \quad 139 \quad 6.56 \quad 19 \quad 21.50 \quad 155 \quad 3.37$ $\begin{array}{lllllll}30 & 617 & 47.31 & 20 & 13.33 & 155 & 39.55\end{array}$

JUL

$\begin{array}{lllll}1 & 1817 \quad 26.42 \quad 19 & 26.98 \quad 155 & 24.65\end{array}$

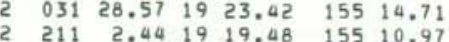

$\begin{array}{rrrrrrrrrrr}.05 & 2.0 & 3.0 & 23 & 0 & 51 & .14 & 4 & .5 & .4 & \text { UEER }\end{array}$

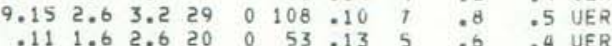

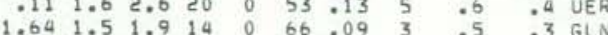
$1.091 .823 .322 \quad 0 \quad 56.11 \quad 5 \quad .540 .36$ LEP

$\begin{array}{lllllllllll}1.76 & 1.6 & 2.2 & 17 & 0 & 66 & .11 & 3 & .5 & .6 & \text { GL }\end{array}$

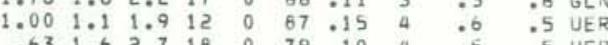

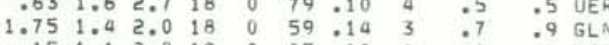
$\begin{array}{lllllllll}15 & 1.1 & 2.0 & 12 & 0 & 85.10 & 4 & 0 & 0\end{array}$

$\begin{array}{lllllllllll}1.52 & 1.5 & 2.1 & 14 & 0 & 87 & 09 & 3 & .5 & .3 & \text { SPC }\end{array}$ $\begin{array}{lllllllllll}.42 & 1.2 & 2.4 & 12 & 0 & 83 & 10 & 4 & .5 & .7 & \text { UEA } \\ 7.78 & 2.0 & 2.5 & 27 & 0 & 75 & 70 & 9 & .5 & .8 & \end{array}$

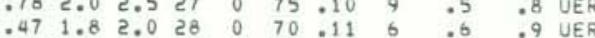

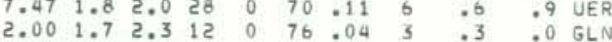

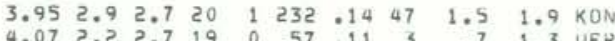

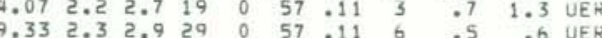

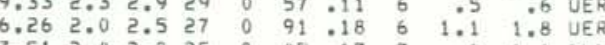

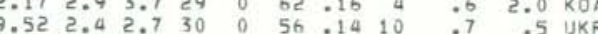
$\begin{array}{lllllllllll}6.13 & 2.4 & 2.9 & 26 & 0 & 76 & .12 & 9 & .7 & .7 & 7 \text { UER }\end{array}$

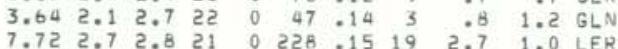
$\begin{array}{lllllllllll}8.33 & 2.2 & 2.7 & 25 & 0 & 77 & .12 & 7 & .7 & 1.1 & \text { UER }\end{array}$

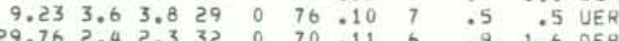
$\begin{array}{llllllllll}29.76 & 2.4 & 2.3 & 32 & 0 & 70.11 & 6 & .9 & 1.6 & \text { OEP }\end{array}$

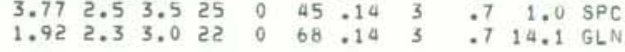
$\begin{array}{lllllllllll}7.31 & 2.8 & 3.4 & 29 & 0 & 157 & .15 & 12 & 1.0 & 1.6 & \text { HLP }\end{array}$

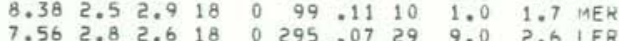

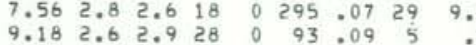

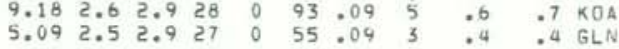

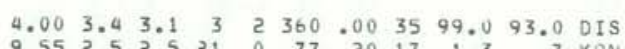

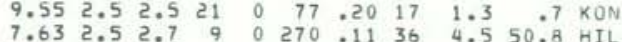

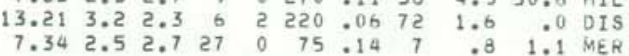
$\begin{array}{lllllllllll}7.83 & 2.6 & 2.9 & 30 & 0 & 123 & .14 & 5 & .8 & 1.0 & \text { KOA }\end{array}$

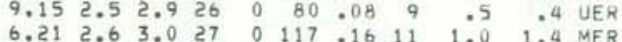

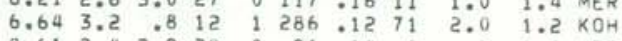
$8.612 .42 .928 \quad 0091.14 \quad 6 \quad .8 \quad 1.0$ UER

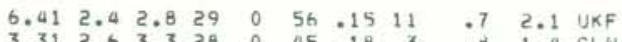

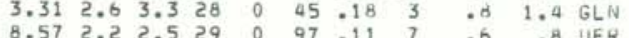

HVO EARTHOUAKE SUMMARY LIST

PAGE 30

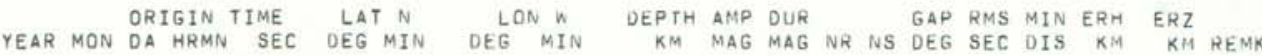

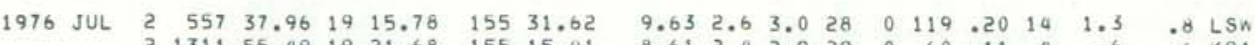

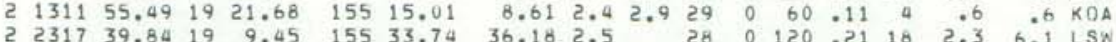

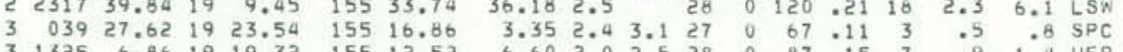

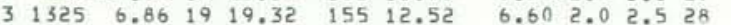

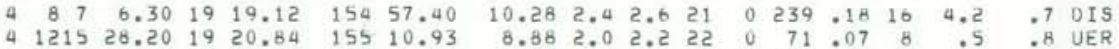

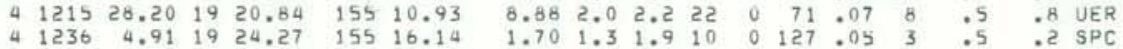

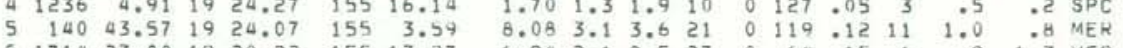

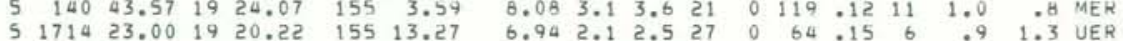

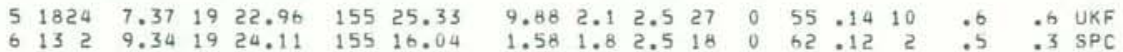

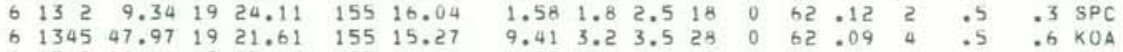

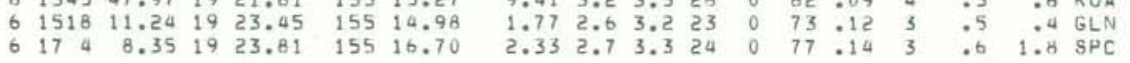

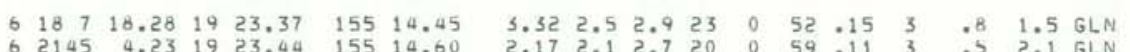

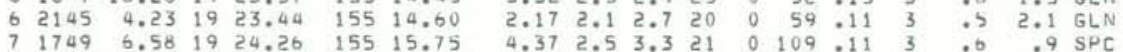

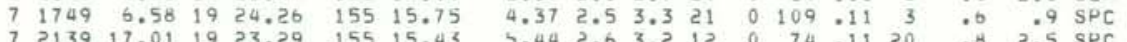

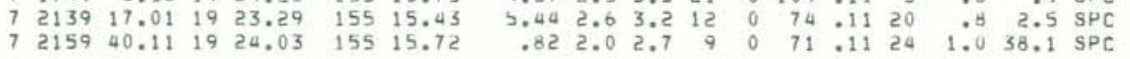

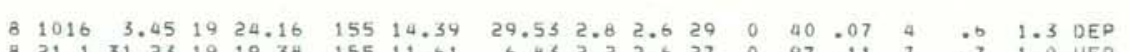

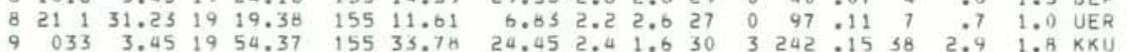

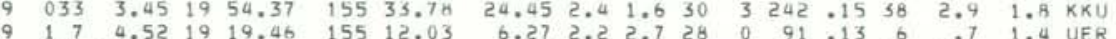

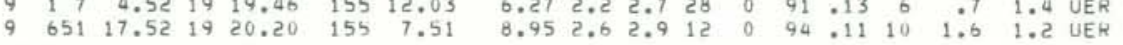

$9172319.72 \quad 1920.40 \quad 155 \quad 6.72$

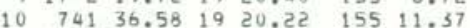
$10 \quad 1150 \quad 24.82 \quad 1920.51 \quad 155 \quad 13.61$

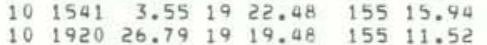

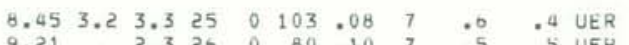

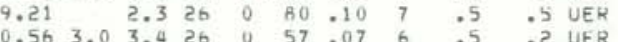

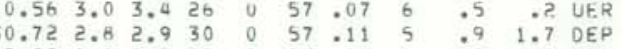
$\begin{array}{lllllllllll}0.72 & 2.8 & 2.9 & 30 & 0 & 57 & .11 & 5 & .9 & 1.7 & \text { DEP } \\ 8.08 & 1.6 & 2.5 & 22 & 0 & 95 & .10 & 6 & .7 & 1.0 & \text { UER }\end{array}$

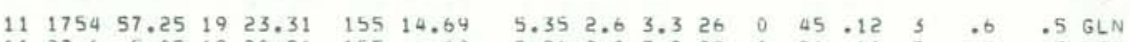

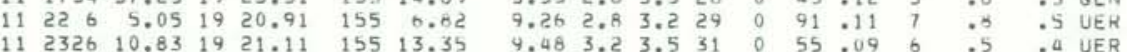

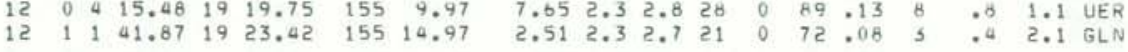
$\begin{array}{llllllllllllllllll}12 & 734 & 39.42 & 19 & 20.96 & 155 & 18.59 & 12.19 & 2.8 & 3.3 & 33 & 0 & 52 & .10 & 5 & .6 & .2 & \text { KOA }\end{array}$

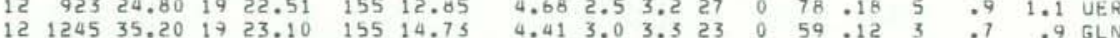

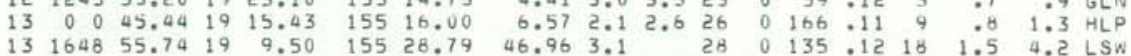
$\begin{array}{lllllllllllllllllll}13 & 18 & 0 & 53.97 & 19 & 19.39 & 155 & 15.68 & 8.06 & 2.1 & 2.6 & 28 & 0 & 91 & .12 & 6 & .7 & 1.1 & K 04\end{array}$

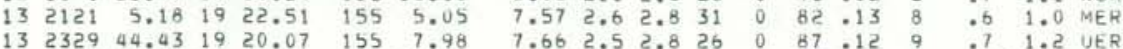

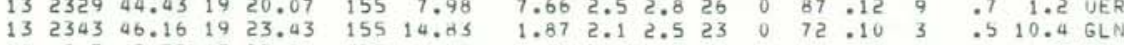

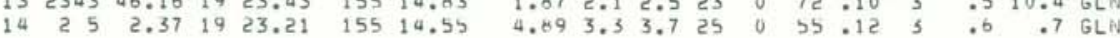
$\begin{array}{lllllllllllllllll}14 & 936 & 54.38 & 19 & 20.30 & 155 & 11.44 & 8.78 & 1.6 & 1.8 & 21 & 0 & 79 & .00 & 7 & .5 & 1.1\end{array}$

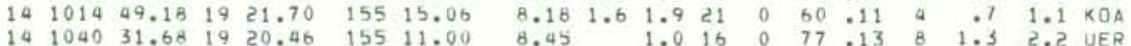




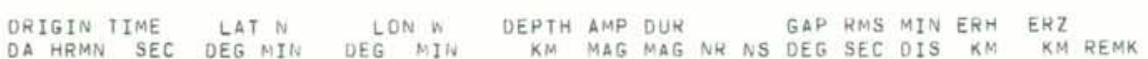

1976 JUL $14 \quad 1044 \quad 58.74 \quad 1919.64 \quad 155 \quad 16.28$ $14104926.621922 .39 \quad 15513.32$ $14 \quad 105755.091923 .97 \quad 15529.69$

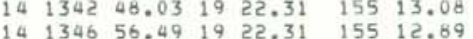

$14 \quad 1348 \quad 44.93 \quad 19 \quad 21.59 \quad 155 \quad 12.87$ $14.135313 .97 \quad 19 \quad 21.5906$ 155

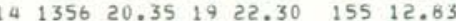
14135947.281922 .20 155 12.85

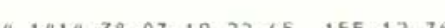
14 191038.0719 az.65 15512.76

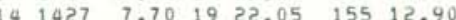
44143540.0519251 .94 155 15.90 $14144240.901922 .25 \quad 15513.12$

$14 \quad 1447 \quad 12.28 \quad 1922.74 \quad 155 \quad 14.12$ $\begin{array}{lllllll}14 & 1448 & 13.27 & 19 & 22.25 & 155 & 12.38 \\ 4 & 1450 & 43.68 & 19 & 22.43 & 155 & 12.59\end{array}$

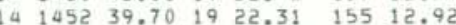
$14 \quad 1458 \quad 44.07 \quad 1922.33 \quad 15512.67$

$14 \quad 15134.251921 .98 \quad 15513.09$ $4 \quad 152025.871922 .48$ 155 13.24

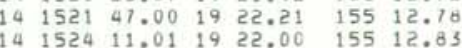

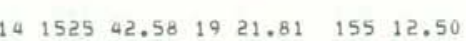
$14 \quad 1527 \quad 55.571921 .91 \quad 155 \quad 12.84$ $14 \quad 1536 \quad 54.46 \quad 1922.19 \quad 15513.04$ $14 \quad \begin{array}{lllll}1554 & 3.24 & 19 & 22.55 \quad 155 \quad 12.64\end{array}$

$\begin{array}{llllllll}14 & 16 & 0 & 26.25 & 19 & 22.35 & 155 & 12.44\end{array}$ 1416156.141922 .72 155 12.51

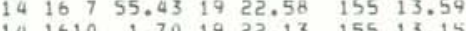
$4.163147 .06 \quad 19223.73 \quad 155 \quad 12.64$

$14 \quad 1625 \quad 19.53 \quad 1922.36 \quad 155 \quad 12.57$

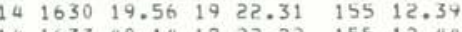
14163340.1419 टट. 2515512.40 14164010.431922 .38 15s 12.64

$14 \quad 1655 \quad 59.12 \quad 1922.63 \quad 155 \quad 12.59$ $\begin{array}{lllllll}14 & 1657 & 17.12 & 19 & 22.29 & 155 & 12.60\end{array}$ $14 \quad 17 \quad 5 \quad 55.96 \quad 1922.55 \quad 155 \quad 12.74$

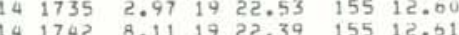

$14 \quad 1744 \quad 53.86 \quad 1922.43 \quad 155 \quad 12.52$

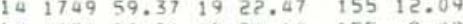

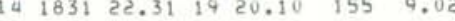

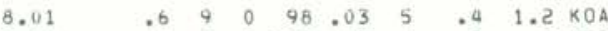

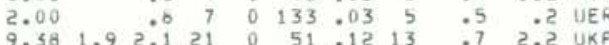

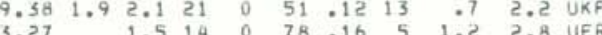

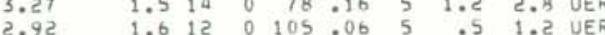
$\begin{array}{lllllllllllll}1.80 & 1.7 & 1.8 & 13 & 0 & 100 & .07 & 5 & .6 & .3 & \text { UER }\end{array}$

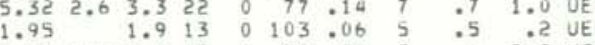
.992 .02 .6 is $0 \quad 70.08$ 5 .6 2.2 UER $\begin{array}{lllllllllll}4.88 & 3.1 & 3.8 & 25 & 0 & 97 & .14 & 8 & .7 & 1.0 & \text { UER }\end{array}$

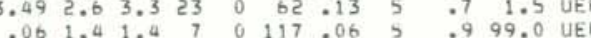

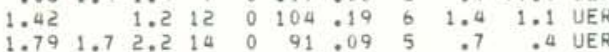
$2.131 .51 .514 \quad 0 \quad$ A5 $.06 \quad 4 \quad .45 .7$ UER $\begin{array}{llllllllll}1.34 & 2.6 & 2.8 & 18 & 0 & 100 & .14 & 8 & .9 & .6 \\ 1.74 & \text { UER }\end{array}$ .80 .3 UER

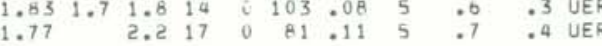
$\begin{array}{llllllllll}1.68 & 1.4 & 10 & 0 & 144 & .05 & 5 & .5 & .3 & .3 \\ 0 & \text { UER }\end{array}$

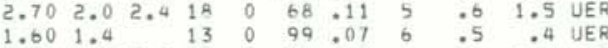
$2.931 .82 .2 \quad 18 \quad 0 \quad 76.1050 .6 \quad 1.4$ UER

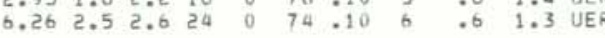
$\begin{array}{lllllllllll}1.02 & 1.4 & 1.1 & 12 & 0 & 107 & .09 & 6 & .7 & .9 & \text { UER }\end{array}$ $\begin{array}{rrrrrrrrrrr}1.35 & 1.9 & 2.7 & 18 & 0 & 91 & .11 & 5 & .6 & .6 & \text { UER } \\ 1.31 & 1.4 & 1.5 & 14 & 0 & 100 & .07 & 6 & .5 & 9 & 4\end{array}$

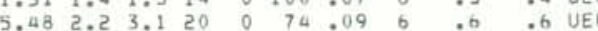
$\begin{array}{lllllllllll}1.42 & 2.3 & 14 & 0 & 113 & .13 & 5 & 1.0 & .6 & .5 & \text { UER }\end{array}$ $\begin{array}{llllllllll}1.54 & 2.3 & 13 & 0 & 112 & .07 & 5 & .7 & .3 & 3 \text { UER }\end{array}$

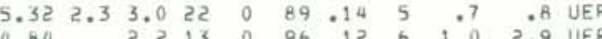

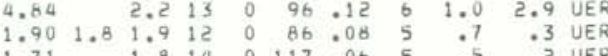
$\begin{array}{lllllllllll}2.03 & 2.2 & 3.0 & 19 & 0 & 82 & .10 & 5 & .5 & 1.7 & \text { UER }\end{array}$ $\begin{array}{lllllllllll}1.67 & 2.6 & 3.4 & 21 & 0 & 84 & .10 & 6 & .6 & .4 & .4 \\ 1.54 & \text { UER }\end{array}$

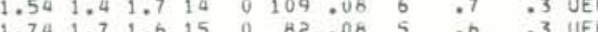

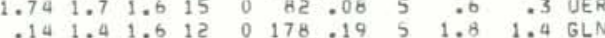

$\begin{array}{lllllllllll}1.66 & 1.7 & 2.0 & 15 & 0 & 115 & .09 & 5 & .7 & .3 & \text { UER }\end{array}$

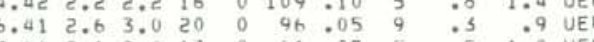

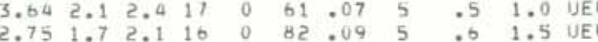

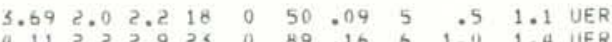

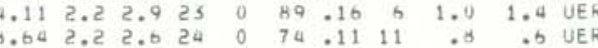
$\begin{array}{lllllllll}1.26 & 1.3 & 13 & 0 & B 2 & .16 & 6 & 1.0 & 1.2 \\ 1.2 & \text { UER }\end{array}$
ORIGIN TIME LAT N LON W
DA HRMN SEC DEG MIN DEG MIN YEAR MON DA HRMN TIMEC DEGTMIN OEG MIN

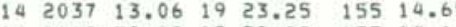
$142119 \quad 30.821922 .99 \quad 155 \quad 14.61$ 14 2ट21 45.09 19 22.19 155 10.04

$\begin{array}{lllllll}14 & 2311 & 54.50 & 19 & 21.36 & 155 & 14.72\end{array}$

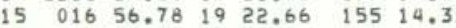
15 2730.441925 .37 155 16.21 $15 \quad 218 \quad 36.34 \quad 1922.16 \quad 155 \quad 12.6$

$\begin{array}{lllllll}15 & 248 & 8.31 & 19 & 22.69 & 155 & 14.71\end{array}$ $15 \quad 313 \quad 19.75 \quad 1922.12 \quad 155 \quad 12.67$

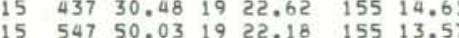

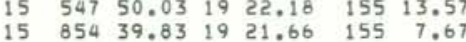

$\begin{array}{llllllll}16 & 111 & 28.75 & 19 & 10.70 & 155 & 32.65\end{array}$ $\begin{array}{lllllll}16 & 447 & 5.75 & 19 & 19.70 & 155 & 11.56\end{array}$ $\begin{array}{llllll}16 & 7 & 4 & 4.17 & 19 & 19.39\end{array}$ $\begin{array}{llllllr}16 & 1128 & 47.24 & 19 & 20.19 & 155 & 6.70 \\ 16 & 2156 & 22.76 & 19 & 23.31 & 155 & 14.52\end{array}$

$\begin{array}{lllllll}17 & 026 & 11.30 & 19 & 20.00 & 155 & 8.59\end{array}$ $\begin{array}{lllllll}18 & 117 & 52.60 & 19 & 19.19 & 155 & 13.9\end{array}$ $\begin{array}{rrrrrrr}18 & 250 & 45.49 & 19 & 20.42 & 155 & 7.93 \\ 18 & 1549 & 51.00 & 19 & 19.15 & 155 & 15.98\end{array}$ $\begin{array}{llllll}18 & 1947 \quad 18.76 \quad 19 & 22.08 \quad 155 \quad 5.06\end{array}$ 18 233! $25.591919 .83 \quad 155 \quad 7.60$ $19051 \quad 15.951919 .89 \quad 155 \quad 7.67$

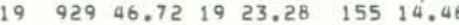

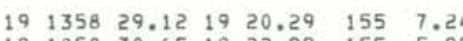

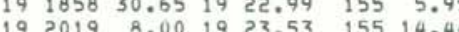
$\begin{array}{llllllll}20 & 023 & 7.67 & 19 & 21.23 & 155 & 13.13\end{array}$ $21 \quad 237 \quad 5.32 \quad 1919.42 \quad 155 \quad 12.7$

$\begin{array}{lllllll}21 & 610 & 5.58 & 19 & 24.04 & 155 & 15.67\end{array}$ $22 \quad 240 \quad 53.051922 .63 \quad 155 \quad 4.62$ 22175313.311919 .92 155 11.19 $\begin{array}{lllllll}23 & 2314 & 4.27 & 19 & 23.45 & 155 & 14.41\end{array}$ $\begin{array}{lllllll}23 & 132 & 50.83 & 19 & 20.68 & 155 & 3.40\end{array}$ $\begin{array}{llllllll}23 & 7 & 2 & 5.36 & 19 & 24.13 & 155 & 15.92\end{array}$ $23,9252.081923 .83$ 154 57.4 23 is a 23.83 19 20.39 155 13.45 $\begin{array}{llllllll}23 & 1938 & 17.12 & 19 & 20.05 & 155 & 0.39\end{array}$ $\begin{array}{llllllll}24 & 0 & 3 & 49.14 & 19 & 20.73 & 155 & 11.23 \\ 24 & 122 & 14.65 & 19 & 13.35 & 155 & 35.54\end{array}$
DEPTH AMP DUR GAP RMS MIN ERH ERZ \begin{tabular}{llllllllll}
.10 & 1.9 & 2.1 & 13 & 0 & 70 & .09 & 3 & .4 & .3 \\
\hline
\end{tabular}

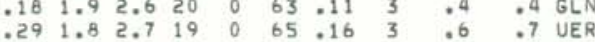

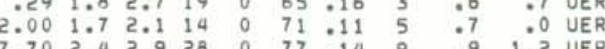

$.221 .4 \quad 1.4 \quad 12 \quad 0 \quad 83.08 \quad 5 \quad .523 .5$ UER

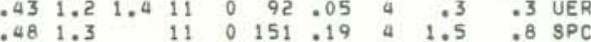

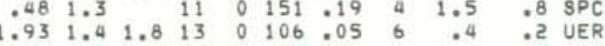

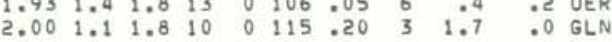
\begin{tabular}{llllllllll}
.33 & 1.9 & 2.8 & 20 & 0 & 69 & .12 & 4 & .5 & .5 \\
\hline & UER
\end{tabular}

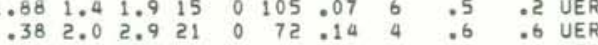
$\begin{array}{lllllllllll}2.00 & 1.3 & 1.8 & 12 & 0 & 87 & : 12 & 5 & : 9 & .0 & \text { UER } \\ 7.81 & 3.3 & 3.5 & 28 & 0 & 72 & .14 & 8 & .7 & .8 & \text { UER }\end{array}$

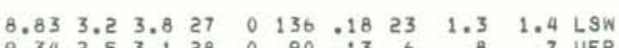
$\begin{array}{lllllllllll}9.34 & 2.5 & 3.1 & 26 & 0 & 90 & .13 & 6 & .8 & .7 & \text { UER }\end{array}$

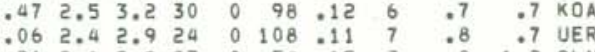

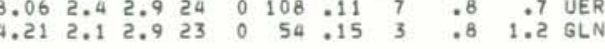
$\begin{array}{lllllllllll}8.42 & 2.3 & 2.8 & 29 & 0 & 76 & .09 & 9 & .6 & .4 & \text { UER }\end{array}$

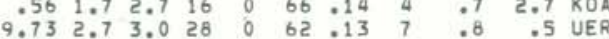

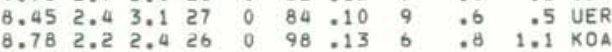
$\begin{array}{llllllllllll}7.59 & 2.1 & 2.2 & 23 & 0 & 90 & .11 & 8 & .7 & 1.1 & \text { MER }\end{array}$ $\begin{array}{llllllll} & .6 & 1.1 & \text { GLN }\end{array}$

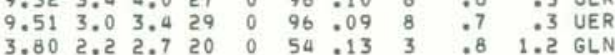
$\begin{array}{llllllllll}6.57 & 2.3 & 2.8 & 29 & 0 & 97 & .14 & 8 & .8 & 1.3 \\ 7 \text { UER }\end{array}$

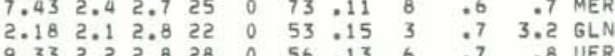

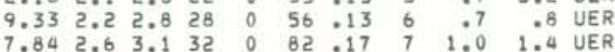
$\begin{array}{lllllllllll}1.31 & 1.8 & 2.7 & 17 & 0 & 113 & .13 & 3 & .6 & .4 & \mathrm{SPC}\end{array}$

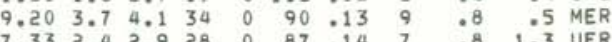

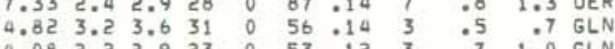
$\begin{array}{llllllllll}4.08 & 2.2 & 2.9 & 23 & 0 & 53 & 12 & 3 & .7 & 1.0\end{array}$ $\begin{array}{llllllllllll}7.71 & 2.8 & 3.3 & 29 & 0 & 100 & .19 & 10 & 1.2 & .7 & \text { MER }\end{array}$

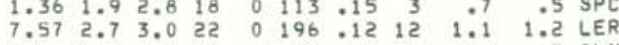

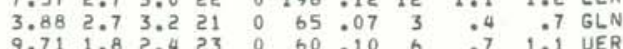

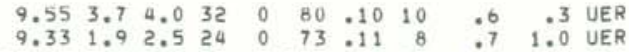

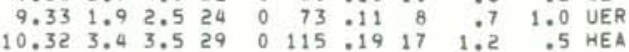




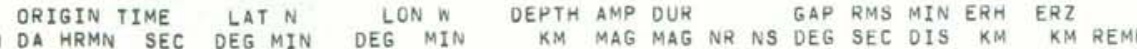

1976 JUL $24 \quad 2053 \quad 44.16 \quad 1923.05 \quad 155 \quad 6.85$ $24 \quad 2346 \quad 30.98 \quad 1923.34 \quad 155 \quad 14.7$ $\begin{array}{lllllll}25 & 114 & 38.87 & 19 & 20.00 & 155 & 13.14\end{array}$ 26 418 4.91 1923.2815514 .50

$\begin{array}{lllllll}26 & 1533 & 8.29 & 19 & 21.82 & 155 & 6.10\end{array}$

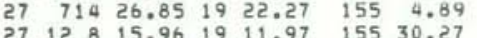

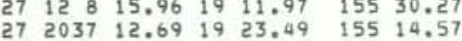
$72214 \quad 6.43 \quad 1921.46 \quad 155 \quad 7.59$

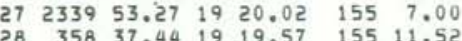

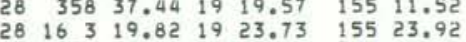
$\begin{array}{rrrrrrr}28 & 1839 & 6.19 & 19 & 19.63 & 155 & 14.28 \\ 28 & 2334 & 22.78 & 19 & 20.13 & 155 & 8.57\end{array}$

$\begin{array}{lllllll}29 & 135 & 59.43 & 19 & 23.64 & 155 & 14.75\end{array}$ $29 \quad 257 \quad 38.83 \quad 1923.54 \quad 155 \quad 14.64$ $\begin{array}{llllllll}29 & 341 & .63 & 19 & 20.96 & 155 & 2.70\end{array}$

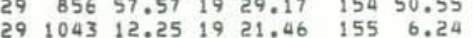

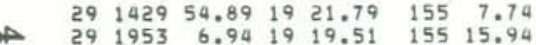

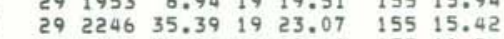

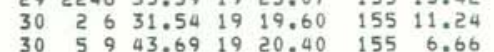
$\begin{array}{llllllll}30 & 8 & 2 & 34.99 & 19 & 20.50 & 155 & 6.76\end{array}$

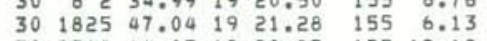
$\begin{array}{lllllll}30 & 2319 & 14.05 & 19 & 20.27 & 155 & 12.19\end{array}$ $\begin{array}{lllllll}31 & 943 & 46.96 & 19 & 21.17 & 155 & 6.20 \\ 31 & 955 & 24.01 & 19 & 23.46 & 155 & 14.73\end{array}$

$\begin{array}{lllllll}31 & 1137 & 1.21 & 19 & 19.48 & 155 & 16.24\end{array}$ $\begin{array}{lllllll}31 & 1312 & 44.26 & 19 & 19.89 & 155 & 16.31\end{array}$ AUG $\begin{array}{lllllllll}1 & 9 & 2 & 55.71 & 19 & 21.57 & 155 & 6.35\end{array}$ $1115133.271923 .19 \quad 15514.53$ $\begin{array}{lllllll}1 & 15 & 1 & 17.48 & 19 & 21.84 & 155 \quad 5.26\end{array}$ $1.64719 .811923 .34 \quad 155 \quad 14.65$

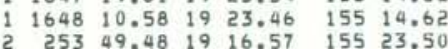

$\begin{array}{lllllll}2 & 637 & 1.42 & 19 & 21.52 \quad 155 \quad 15.11\end{array}$ $\begin{array}{lllllll}3 & 330 & 17.09 & 19 & 19.35 & 155 & 12.62\end{array}$ 4. $2124 \quad 8.35 \quad 1923.45 \quad 155 \quad 14.47$ 4224
$40.391923 .42 \quad 15514.57$

$\begin{array}{lllllll}5 \quad 957 & 20.60 & 19 & 24.14 & 155 & 15.8\end{array}$ $\begin{array}{lllllll}6 & 338 & 55.38 & 19 & 22.71 & 155 & 5.58 \\ 6 & 755 & 40.42 & 19 & 22.5 & 155 & 5.92\end{array}$ $\begin{array}{lllllllllll}8.47 & 2.9 & 3.1 & 29 & 0 & 65 & .08 & 9 & .4 & .6 & \text { GLN }\end{array}$

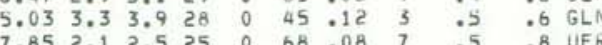

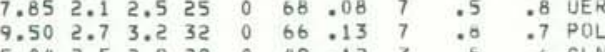

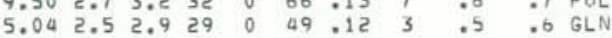

$\begin{array}{lllllllllll}7.86 & 2.7 & 2.9 & 30 & 0 & 79 & .11 & 7 & .7 & .6 & \text { UER } \\ 9.39 & 4.0 & 4.1 & 32 & 0 & 83 & .12 & 9 & 99 & .5 & \text { MEF }\end{array}$

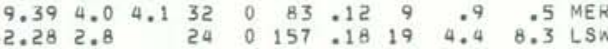

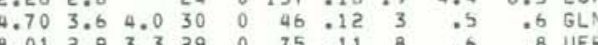
$\begin{array}{llllllllll}7.60 & 2.4 & 2.9 & 25 & 0 & 107 & .11 & 7 & .6 & .9 \\ 0 \text { UER }\end{array}$

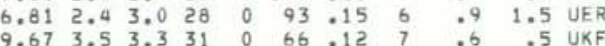

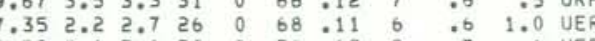

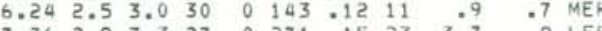

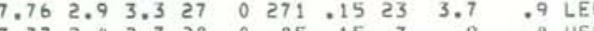

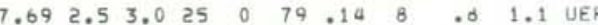

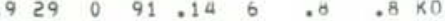

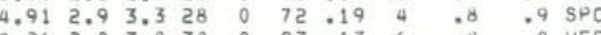

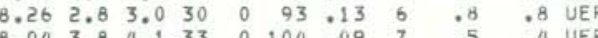

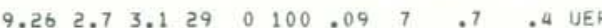
$\begin{array}{llllllllllll}8.59 & 2.8 & 3.4 & 31 & 0 & 89 & .12 & 7 & .8 & .5 & .5 \\ 8\end{array}$

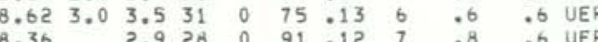
$\begin{array}{lllllllllll}8.36 & 2.9 & 28 & 0 & 91 & .12 & 7 & .8 & .6 & 6 & 0 \\ 5.17 & 3.1 & 3.3 & 20 & 0 & 64 & .10 & 5 & .5 & .6 & 6 L R\end{array}$ $\begin{array}{lllllllllll}8.80 & 2.3 & 2.8 & 27 & 0 & 95 & .11 & 5 & .7 & .8 & K 0 \mathrm{~A}\end{array}$

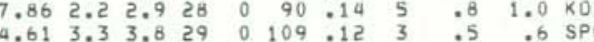

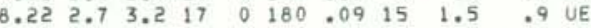
$1.763 .03 .321 \quad 0 \quad 46.1233 \quad .631 .5$ GLN \begin{tabular}{lllllllllll}
8.16 & 3.4 & 3.4 & 29 & 0 & 78 & .11 & 8 & .7 & .5 \\
\hline
\end{tabular} $\begin{array}{llllllllll}1.46 & 2.5 & 18 & 0 & 68 & .11 & 3 & .5 & .4 & 0 L\end{array}$ $\begin{array}{rrrrrrrrrrr}1.98 & 2.5 & 2.8 & 16 & 0 & 70 & : 12 & 3 & .5 & 23.4 & \text { GL } \\ 5.36 & 2.3 & 2.6 & 26 & 0 & 134 & : 16 & 9 & .9 & 1.2 & \text { SWR }\end{array}$

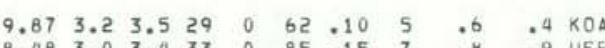

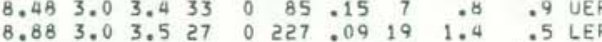
$\begin{array}{lllllllllll}4.18 & 2.3 & 2.9 & 24 & 0 & 53 & .14 & 3 & .7 & 1.0 & \text { GLN }\end{array}$

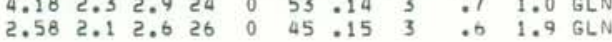
$\begin{array}{lllllllllllll}1.54 & 2.2 & 3.0 & 19 & 0 & 108 & .11 & 3 & .5 & .3 & \text { SPC }\end{array}$

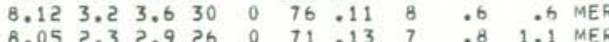

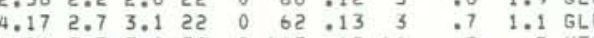

HVO EARTHQUAKE SUMMAKY LIST

PAGE 30

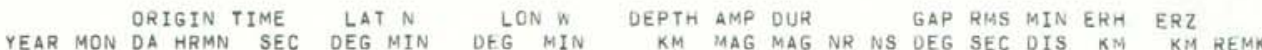

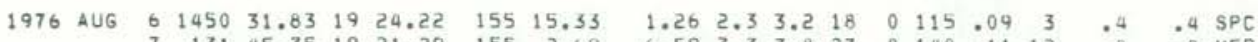

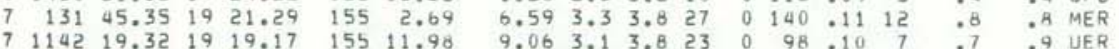

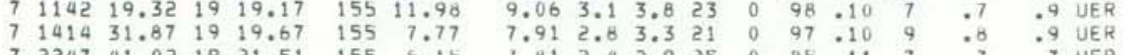
$72247 \quad 41.02 \quad 1921.51 \quad 155 \quad 6.15$

$8 \quad 510 \quad 32.20 \quad 1923.95 \quad 155 \quad 15.19$

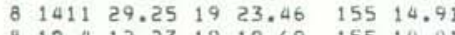
819412.231919 .69 155 10.01 $913139.941925 .54 \quad 15514.42$

$91757 \quad 33.62 \quad 1920.97 \quad 15511.27$
10

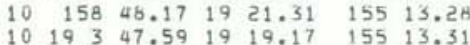
$\begin{array}{llllllll}10 & 19 & 3 & 47.59 & 19 & 19.17 & 155 & 13.31 \\ 11 & 119 & 4.60 & 19 & 23 & 4 & 155 & 15\end{array}$

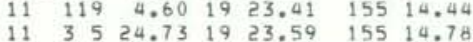

$\begin{array}{lllllll}11 & 1630 & 11.09 & 19 & 20.48 & 155 & 11.50\end{array}$ $\begin{array}{lllllll}12 & 720 & 18.70 & 19 & 19.37 & 155 & 13.61\end{array}$ $\begin{array}{lllllll}13 & 753 & 18.24 & 19 & 28.98 & 155 & 2.67 \\ 13 & 1624 & 11.76 & 19 & 42.73 & 155 & 2.59\end{array}$

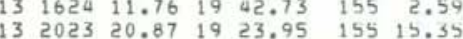

$\begin{array}{lllllll}14 & 020 & 11.88 & 19 & 24.07 & 155 & 15.72\end{array}$ $\begin{array}{lllllll}14 & 231 & 0.47 & 19 & 23.50 & 155 & 14.60\end{array}$ $\begin{array}{lllllll}14 & 938 & 48.16 & 19 & 22.07 & 155 & 7.17\end{array}$

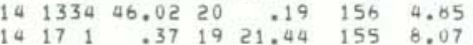

$14 \quad \begin{aligned} & 1753 \quad 34.07 \quad 1920.38 \quad 155 \quad 12.60 \\ & 1453\end{aligned}$ $\begin{array}{lllllll}4 & 2237 & 8.83 & 19 & 19.18 & 155 & 11.9\end{array}$ $\begin{array}{lllllll}15 & 117 & 51.35 & 19 & 20.19 & 155 & 13.20\end{array}$

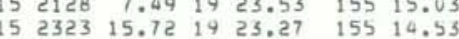

$\begin{array}{llllllll}16 & 0 & 6 & 58.48 & 19 & 23.22 & 155 & 14.56\end{array}$ $\begin{array}{lllllll}16 & 231 & 8.43 & 19 & 21.89 & 155 & 3.90\end{array}$

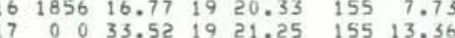

$\begin{array}{lllllll}17 & 423 & 39.90 & 19 & 27.44 & 154 & 52.91\end{array}$

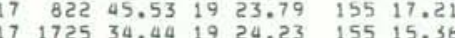

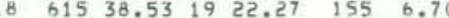
$\begin{array}{lllllll}18 & 642 & 48.37 & 19 & 22.53 & 155 & 6.58\end{array}$

$\begin{array}{lllllll}18 & 1129 & 42.91 \quad 19 & 23.42 & 155 & 14.19\end{array}$ $\begin{array}{lllllll}18 & 1140 & 29.36 & 19 & 23.53 & 155 & 14.78\end{array}$ $18 \quad 1348 \quad 49.33 \quad 1931.82$ 155 27.58 $\begin{array}{llllllll}18 & 1541 & 6.94 & 19 & 20.11 & 155 & 13.04\end{array}$ $\begin{array}{llllllll}19 & 12 & 7 & 42.51 & 19 & 19.47 & 155 & 11.80\end{array}$

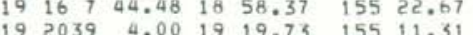

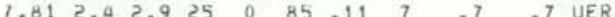

$\begin{array}{lllllllllll}1.93 & 1.9 & 2.8 & 17 & 0 & 58 & .09 & 3 & .3 & 18.4 & \text { SPC }\end{array}$

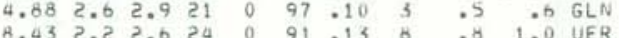

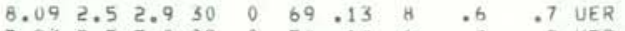

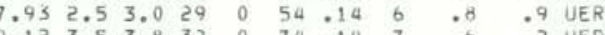

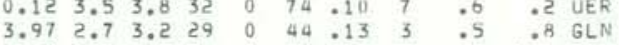

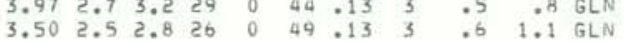

$\begin{array}{lllllllllll}6.20 & 2.7 & 3.3 & 29 & 0 & 76 & .14 & 7 & .8 & 1.0 & \text { UER }\end{array}$

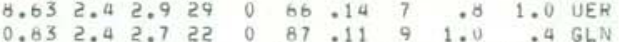

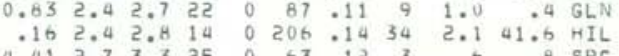

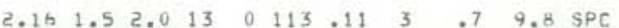

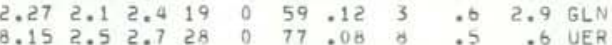

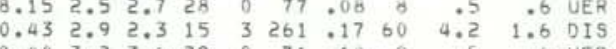
8.043 .523 .925

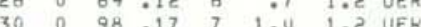

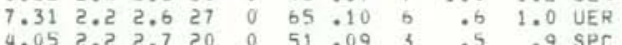

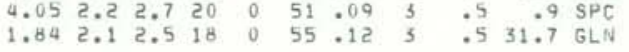

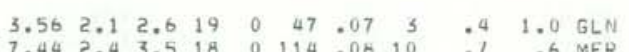

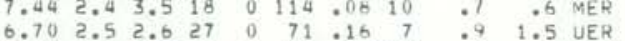

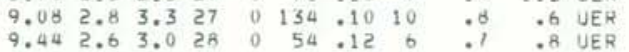
$\begin{array}{lllllllllll}7.93 & 2.7 & 3.0 & 26 & 0 & 252 & .20 & 20 & 3.6 & 1.0 & \text { LER }\end{array}$

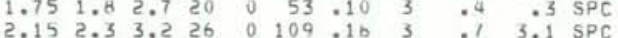

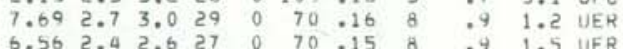

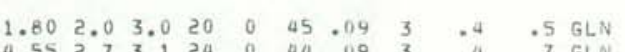

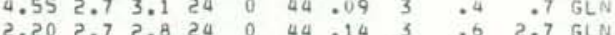

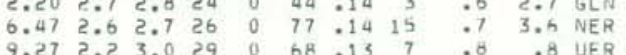

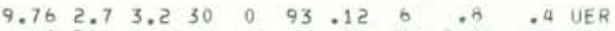

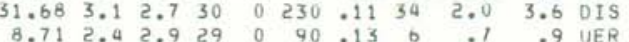

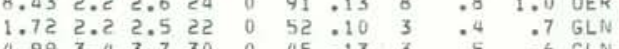




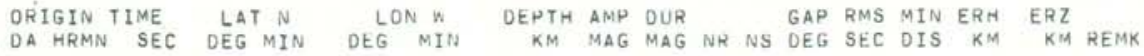

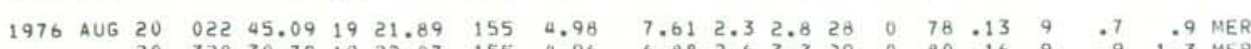

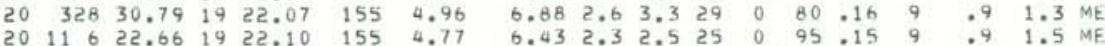

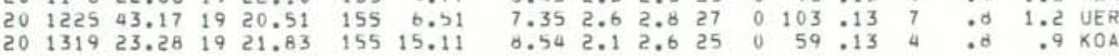

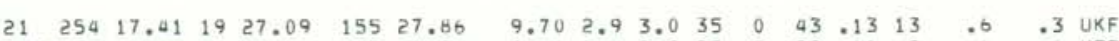

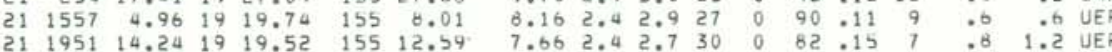

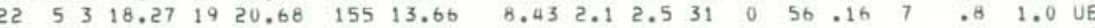

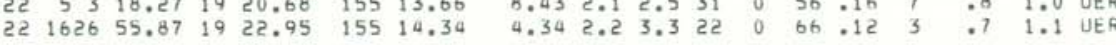

$\begin{array}{llllll}22 & 179 & 18.33 \quad 19 & 24.93 \quad 155 \quad 24.87\end{array}$ $221726 \quad 14.98 \quad 1920.50 \quad 155 \quad 7.43$ $22 \quad 1736 \quad 37.78 \quad 1920.76 \quad 155 \quad 13.61$ $23183423.301923 .49 \quad 15521.71$

$\begin{array}{lllllll}23 & 2349 & 7.25 & 19 & 20.17 & 155 & 7.32\end{array}$ $242923.731920 .94 \quad 15511.04$ $241937 \quad 1.901917 .51 \quad 15521.61$ $26 \quad 319 \quad 43.60 \quad 1921.78 \quad 155 \quad 15.00$

$\begin{array}{lllllll}26 & 710 & 45.38 & 19 & 21.62 & 155 & 8.22\end{array}$ $\begin{array}{lllllll}26 & 736 & 13.04 & 19 & 19.66 & 155 & 15.91\end{array}$ 27 050 44.2919 23.17 1550.64

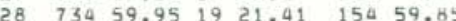

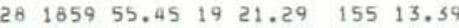
28181747.5219 टर.0द 1550.76 29133142.731920 .00 154 58.41 $\begin{array}{lllllll}29 & 1549 & 6.23 & 19 & 21.24 & 155 & 1.53\end{array}$

$\begin{array}{llllllll}29 & 23 & 7 & 14.28 & 19 & 20.27 & 155 & 13.78\end{array}$

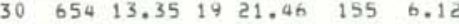

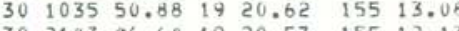
$30214346.641920 .57 \quad 155 \quad 12.13$

$\begin{array}{lllllll}31 & 258 & 14.50 & 19 & 20.57 & 155 & 6.67\end{array}$ $\begin{array}{lllllll}31 & 1041 & 8.47 & 19 & 23.42 & 155 & 29.67\end{array}$ $\begin{array}{lllllll}31 & 1120 & 3.78 & 19 & 20.57 & 155 & 7.40\end{array}$ $312041 \quad 8.04 \quad 1919.60 \quad 15511.81$

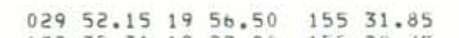

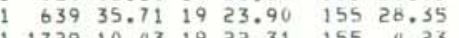
$1 \quad 172910.43 \quad 1922.31 \quad 155 \quad 4.25$

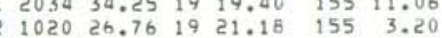
$\begin{array}{llllllll}2 & 20 & 1 & 49.06 & 19 & 2.71 & 154 & 56.44\end{array}$

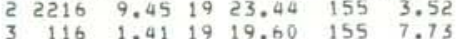

$\begin{array}{llllllllllll}9.48 & 2.4 & 2.9 & 23 & 0 & 58 & .10 & 9 & .5 & .8 & \text { UKF }\end{array}$

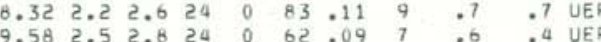

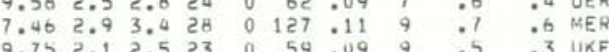
$\begin{array}{lllllllllll}7.38 & 2.4 & 2.9 & 29 & 0 & 98 & .10 & \text { A } & .0 & .8 & .8 \\ 7 & \text { UEH }\end{array}$

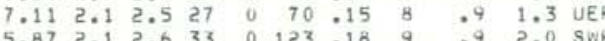
$\begin{array}{rrrrrrrrrrr}5.87 & 2.1 & 2.6 & 33 & 0 & 123 & .18 & 9 & .9 & 2.0 & \text { SWH } \\ 8.45 & 2.3 & 2.7 & 27 & 0 & 85 & .12 & 7 & .7 & .8 & .8\end{array}$

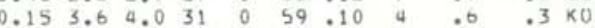
$\begin{array}{llllllllll}6.67 & 2.2 & 2.7 & 26 & 0 & 68 & .09 & 9 & .5 & .5 \\ 0.5 E R\end{array}$

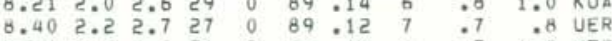

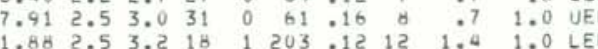

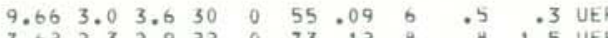
$7.40 \quad 2.112 .121100184 .19$ 13 2.2 1.4 MER $\begin{array}{lllllllllll}8.39 & 2.0 & 2.4 & 17 & 0 & 69 & .09 & 6 & .6 & .9 & \text { UER }\end{array}$

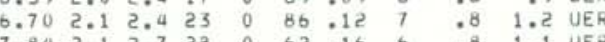

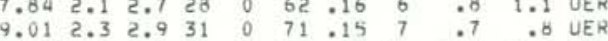

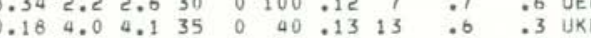

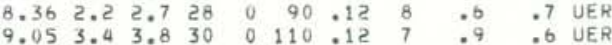

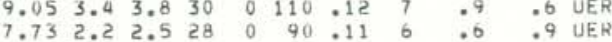

$\begin{array}{lllllllllll}3.73 & 2.8 & 2.5 & 36 & 3 & 158 & .17 & 33 & 1.0 & 20.6 & \mathrm{KOH}\end{array}$

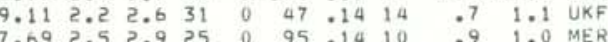

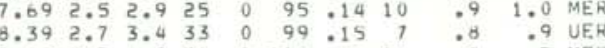

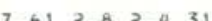

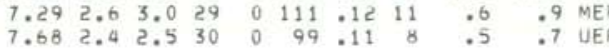

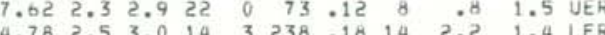

ORIGIN TIME LATN LON W YEAR MON DA HRMN SEC DEG MIN DEG MIN

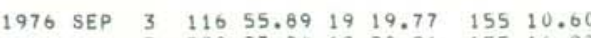

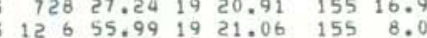

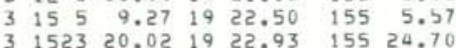
$3 \quad 154328.17 \quad 1923.86 \quad 155 \quad 5.40$

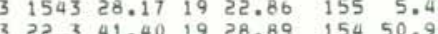

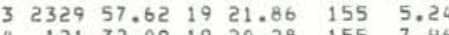

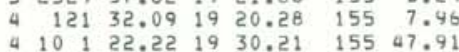

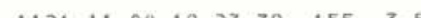
\begin{tabular}{l}
$1310 \quad 54.24 \quad 19 \quad 19.79 \quad 155 \quad 16.3$ \\
\hline
\end{tabular}

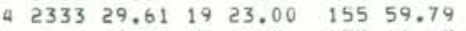

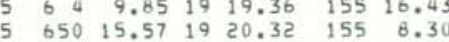

$\begin{array}{lllllll}5 & 1454 & 26.70 & 19 & 46.13 \quad 155 & 46.79\end{array}$ 6
6 $42828.36 \quad 1920.77 \quad 155 \quad 10.00$ 54118.571923 .51 155 2.30
6 $6 \quad 758 \quad 26.19 \quad 19 \quad 19.71 \quad 15511.31$
6

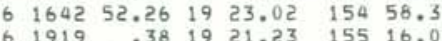

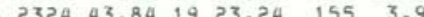
$31257.16 \quad 1922.00$
$755 \quad 8.50$

$\begin{array}{lllllll}7 & 1418 & 14.00 & 19 & 21.36 & 155 & 5.94\end{array}$

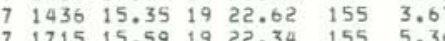
$7175410.591922 .34 \quad 155 \quad 5.36$ $\begin{array}{llllll}72124 & 48,74 & 19 & 22.37 \quad 155 & 4.84\end{array}$

$\begin{array}{llllllll}7 & 22 & 1 & 16.28 & 19 & 22.20 & 155 & 4.73\end{array}$ $\begin{array}{llllllll}8 & 4 & 1 & 55.52 & 19 & 18.45 & 155 & 20.83\end{array}$ 863819.241921 .45 155 6.19 $8 \quad 103358.051919 .99 \quad 155 \quad 5.05$

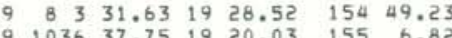

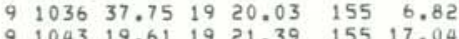

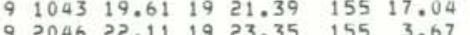
$10204622.11 \quad 1923.35$ 155 3.67

$\begin{array}{lllllll}10 & 424 & 23.00 & 19 & 17.83 & 155 & 16.92\end{array}$ $\begin{array}{llllllll}10 & 625 & 18.62 & 18 & 48.97 & 155 & 13.54 & 36\end{array}$ $\begin{array}{lllllll}10 & 858 & 35.22 & 19 & 20.45 & 155 & 12.82 \\ 10 & 2157 & 47.47 & 19 & 20.61 & 155 & 8.94\end{array}$

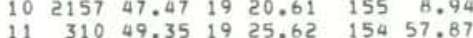

$\begin{array}{lllllll}11 & 429 & 26.78 & 19 & 21.36 & 155 & 3.39\end{array}$

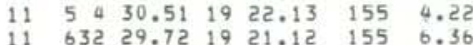

DEPTH AMP DUR GAP RMS MIN ERH ERI $\begin{array}{lllllllllll}3.90 & 3.1 & 3.5 & 33 & 0 & 91 & .12 & 7 & .7 & .6 & \text { UER }\end{array}$

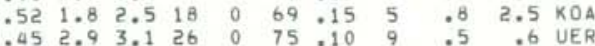

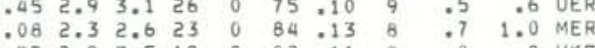
$9.572 .93 .519 \quad 0 \quad 82.11$ \% 94.98 UKF $\begin{array}{lllllllllll}8.23 & 2.8 & 3.2 & 25 & 0 & 78 & .12 & 8 & .7 & 1.0 & \text { MER }\end{array}$

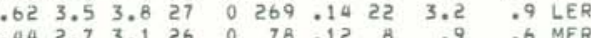

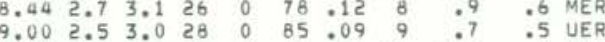

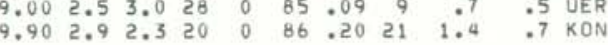
$\begin{array}{llllllllllll}8.81 & 2.6 & 2.9 & 21 & 0 & 111 & .10 & 11 & .9 & .5 & .5 E R\end{array}$

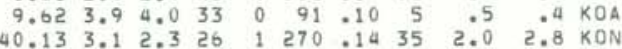

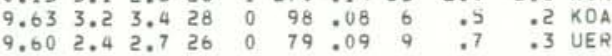
$\begin{array}{lllllllllll}12.44 & 3.0 & 2.6 & 28 & 0 & 146 & .20 & 34 & 1.6 & 1.0 & \text { KON }\end{array}$

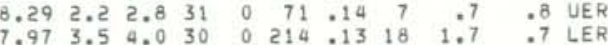

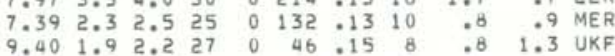
$\begin{array}{llllllllllll}9.22 & 2.4 & 3.0 & 26 & 0 & 91 & .12 & 6 & .7 & .8 & \text { UER }\end{array}$

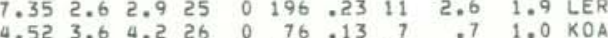

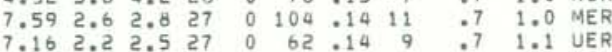
\begin{tabular}{llllllllll}
6.10 & 2.3 & 2.7 & 28 & 0 & 88 & .14 & 7 & .8 & 1.4 \\
\hline & MER
\end{tabular}

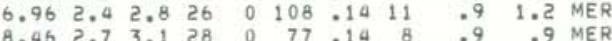

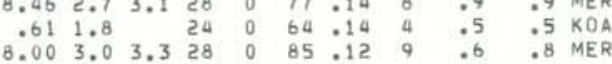

$\begin{array}{rrrrrrrrrr}8.28 & 3.5 & 4.0 & 32 & 0 & 85 & .12 & 9 & .7 & .6 \\ 9.5 & \text { MER }\end{array}$

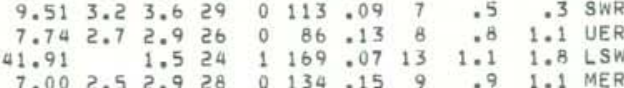

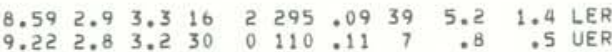

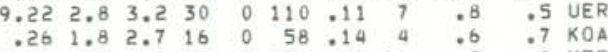

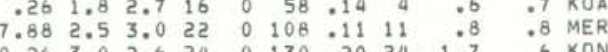
$\begin{array}{llllllllllll}10.26 & 3.0 & 2.6 & 24 & 0 & 130 & .20 & 24 & 1.7 & .6 & \mathrm{KON}\end{array}$

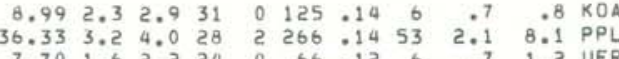

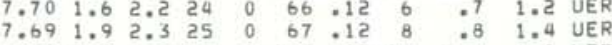
$1.302 .02 .520 \quad 2 \quad 168.1911 \quad 1.4 \quad 1.9$ LER

$\begin{array}{lllllllllll}0.13 & 2.1 & 2.5 & 24 & 0 & 112 & .12 & 11 & .7 & 1.2 & \text { MER }\end{array}$

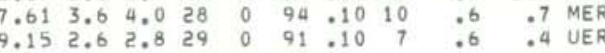


$\begin{array}{llllll}\text { ORIGIN TIME } & \text { LAT N } & \text { LON W } & \text { DEPTH AMP DUR } & \text { GAP RMS MIN ERH ERZ } \\ \text { DA HRMN SEC DEG MIN } & \text { DEG MIN } & \text { KM MAG MAG NR NS DEG SEC DIS KM KM REMK }\end{array}$ YEAR MON DA HRMN SEC DEG MIN DEG MIN $11 \quad 1340 \quad 15.00 \quad 1922.56 \quad 155 \quad 3.02$ $\begin{array}{llllllll}11 & 18 & 5 & 40.11 & 19 & 22.71 & 155 & 26.87\end{array}$ $112352 \quad 35.001920 .23 \quad 155 \quad 13.29$

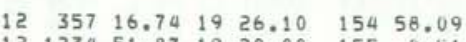
$12123451.871920 .08 \quad 155 \quad 8.51$

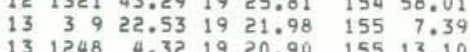
$13 \quad 1248 \quad 4.321920 .90 \quad 15513.10$

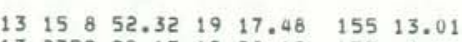

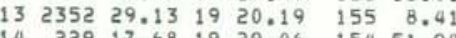

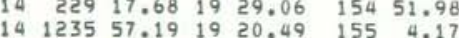

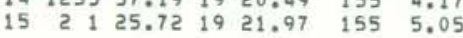

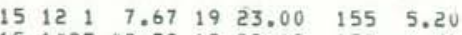
$15142548.591922 .19 \quad 155 \quad 6.77$ 16 61722.411922 .54 155 26.11

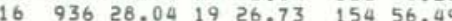

$\begin{array}{lllllll}16 & 1458 & 38.48 & 19 & 23.15 & 155 & 25.26\end{array}$

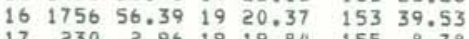

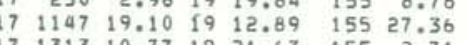

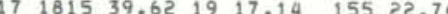
$17193010.72 \quad 1924.74 \quad 155 \quad 14.95$ $\begin{array}{llllllll}18 & 2 & 1 & 45.88 & 19 & 22.28 & 155 & 4.69 \\ 18 & 1114 & 58.11 & 19 & 19.28 & 155 & 13.35\end{array}$ $\begin{array}{lllll}18 & 1857 \quad 17.42 \quad 19 & 19.56 & 155 & 12.13\end{array}$

$\begin{array}{lllllll}18 & 1914 & 51.75 & 19 & 20.52 & 155 & 4.87\end{array}$

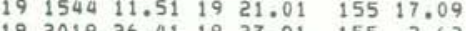

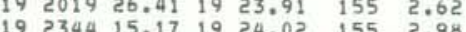

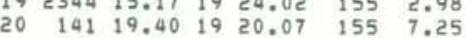

$\begin{array}{lllllll}20 & 1716 \quad 38.31 & 19 & 21.98 & 155 & 6.57\end{array}$ $\begin{array}{lllllll}20 & 1730 & 8.99 & 19 & 21.40 & 155 & 30.29\end{array}$ $\begin{array}{lllllll}20 & 1928 & 25.90 & 19 & 21.88 & 155 & 16.12\end{array}$

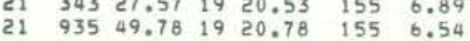
$\begin{array}{lllllll}21 & 1621 & 37.29 & 19 & 21.12 & 155 & 6.98\end{array}$

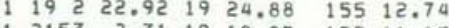
$\begin{array}{lllllll}21 & 2153 & 2.31 & 19 & 19.27 & 155 & 16.15\end{array}$

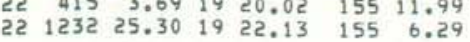
$\begin{array}{llllll}22 & 1718 & 48.04 & 19 & 21.32 \quad 155 \quad 13.23\end{array}$ $24 \quad 123 \quad 38.61 \quad 1920.03 \quad 155 \quad 8.68$ $\begin{array}{lllllllllll}7.23 & 1.8 & 2.1 & 15 & 0 & 171 & .13 & 13 & 1.5 & 1.5 \\ 7 & \text { LER }\end{array}$

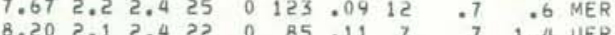

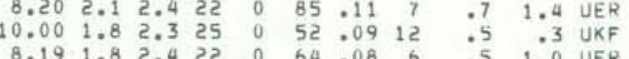
$\begin{array}{lllllllllll}3.46 & 2.1 & 2.3 & 14 & 0 & 159 & .13 & 11 & 1.4 & 1.9 & \text { LER }\end{array}$

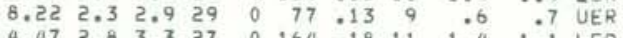

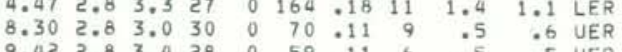

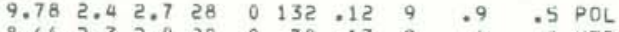

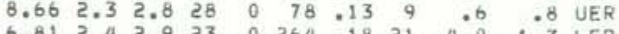

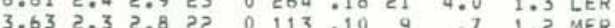

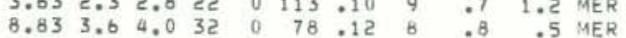
$\begin{array}{lllllllllll}7.67 & 2.9 & 3.4 & 30 & 0 & 83 & .12 & 8 & .6 & .8 & \text { MER }\end{array}$ 7.10 .

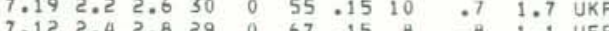

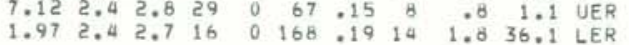

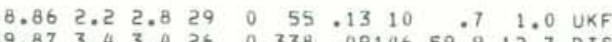

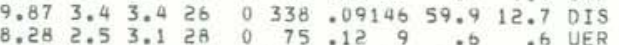
$\begin{array}{lllllllll}7.43 & 2.2 & 2.7 & 30 & 0 & 122 & .17 & 7\end{array}$

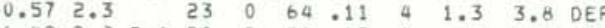

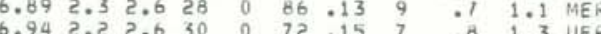

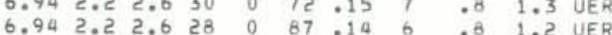
$\begin{array}{llllllllll}5.63 & 2.4 & 2.7 & 28 & 0 & 114 & .13 & 8 & .8 & 1.2 \\ 1.2 & M E R\end{array}$ 7.5003 .33 .013 2 $117.1010 \quad 1.0$

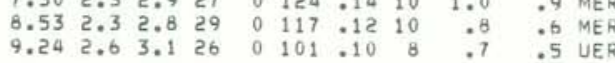

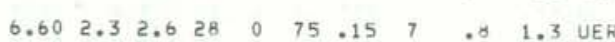

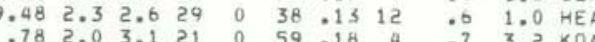

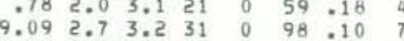

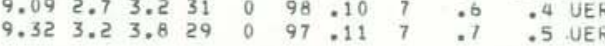
$\begin{array}{lllllllllll}6.55 & 2.4 & 2.8 & 25 & 0 & 86 & .15 & 7 & .9 & 1.6 & \text { UER }\end{array}$

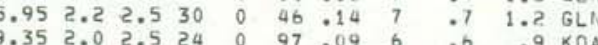

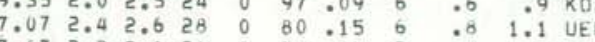

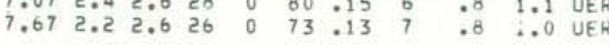

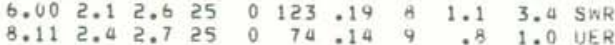

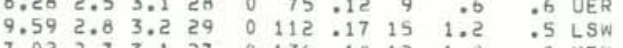
$\begin{array}{lllllllllllll}9.47 & 2.2 & 2.7 & 27 & 0 & 55 & .12 & 6 & .6 & .7 & \text { UER }\end{array}$
HVO EARTHQUAKE SUMMARY LIST

PAGE 38 ORIGIN TIME LATN LON $N$ DEPIH AMP DUR GAP RMS MIN ERH ER

KM MAG MAG NR NS DEG SEC DIS KH ERZ REMK

$\begin{array}{llllllll}1976 & \text { SEP } 24 & 157 & 2.29 & 19 & 21.20 & 155 & 13.37\end{array}$ $\begin{array}{lllllll}24 & 1228 & 18.91 & 19 & 19.90 & 155 & 8.12\end{array}$ $24225131.281920 .61 \quad 155 \quad 6.68$

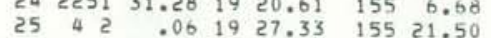

$\begin{array}{lllllll}25 & 1554 & 55.88 & 19 & 26.77 & 155 & 23.09\end{array}$ $\begin{array}{lllllll}25 & 1720 & 50.41 & 19 & 20.43 & 155 & 6.44 \\ 26 & 650 & 44.95 & 19 & 24.75 & 155 & 26.23\end{array}$ $26 \quad 65044.951924 .75 \quad 15526.23$ 26183420.051920 .96 155 11.21

$\begin{array}{lllllll}27 & 846 & 57.71 & 19 & 25.51 & 155 & 25.78\end{array}$

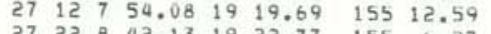

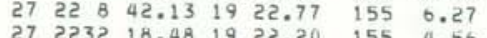

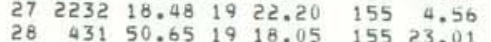

$\begin{array}{lllllll}28 & 914 & 53.40 & 19 & 20.00 & 155 & 13.13\end{array}$ $\begin{array}{lllllllll}28 & 10 & 2 & 49.26 & 19 & 20.24 & 155 & 8.04\end{array}$ $\begin{array}{lllllll}28 & 1437 & 30.09 & 19 & 19.38 & 155 & 13.96\end{array}$

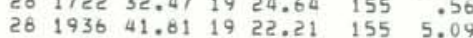

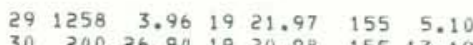
$30 \quad 1532 \quad 46,37 \quad 19$

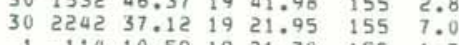

$16844.9219 \quad 19.51 \quad 15512.11$

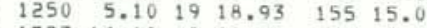

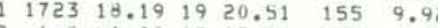

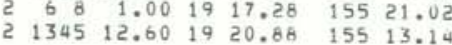

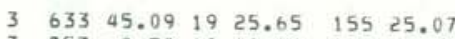
$\begin{array}{llllllll}3 & 753 & 8.56 & 19 & 19.79 & 155 & 7.26\end{array}$

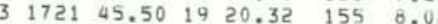
$418 \quad 52.57 \quad 1920.69 \quad 155 \quad 6.51$

$434 \quad 4.72 \quad 19 \quad 20.09 \quad 155 \quad 12.09$ $\begin{array}{llllll}558 & 50.57 & 19 & 20.11 & 155 & 6.59\end{array}$ $516750.6720 \quad .63 \quad 155 \quad 32.92$
6 $6 \quad 822 \quad 12.93 \quad 19 \quad 18,17 \quad 155 \quad 23.49$

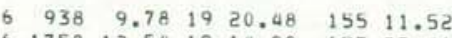
$\begin{array}{llllll}1758 & 12.54 & 19 & 14.99 & 155 & 11.52\end{array}$ $31137.96 \quad 19$ az.68 155 5.39 $\begin{array}{llll}6 \quad 3 \quad 44.37 \quad 19 & 20.77 \quad 155 \quad 5.39\end{array}$

$\begin{array}{lllllll}7 & 1051 & 7.96 & 19 & 20.25 & 155 & 9.06\end{array}$

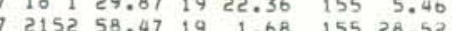

$9.662 .6 \quad 3.135 \quad 0 \quad 54.11 \quad 6 \quad 060.3$ UER

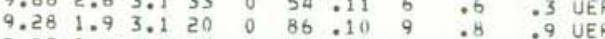

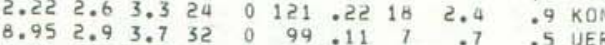
$\begin{array}{lllllllllll}8.95 & 2.9 & 3.7 & 32 & 0 & 99 & .11 & 7 & .7 & .5 & .5 \\ 6.01 & 2.3 & 3.3 & 24 & 0 & 78 & .15 & 9 & .7 & 2.2 & \text { UKF }\end{array}$ $\begin{array}{lllllllllllll}0.24 & 3.0 & 3.2 & 23 & 0 & 74 & .12 & 11 & .6 & .9 & \text { UKF }\end{array}$

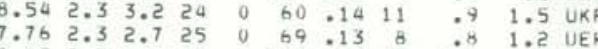

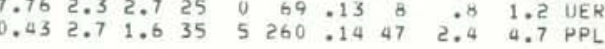

$\begin{array}{lllllllllllll}0.29 & 2.9 & 3.2 & 32 & 0 & 48 & .12 & 11 & .6 & .3 & \text { UKF }\end{array}$

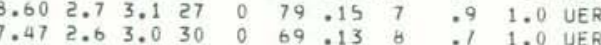

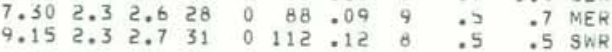

$\begin{array}{lllllllllll}10.04 & 3.7 & 4.2 & 34 & 0 & 68 & .10 & 7 & .6 & .2 & \text { UER }\end{array}$

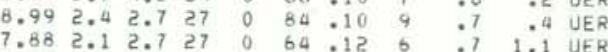

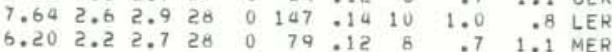
$\begin{array}{lllllllllll}8.69 & 3.3 & 3.8 & 29 & 0 & 77 & .11 & 8 & .8 & .5 & \text { MER }\end{array}$

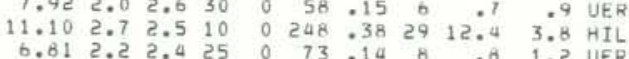

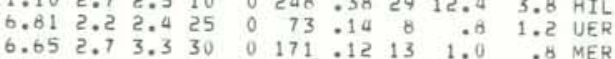
$\begin{array}{lllllllllll}8.78 & 2.4 & 3.0 & 27 & 0 & 89 & .14 & 6 & .9 & 1.1 & \text { UER }\end{array}$

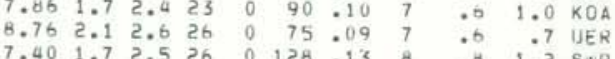
$\begin{array}{lllllllllll}.40 & 1.7 & 2.5 & 26 & 0 & 128 & .13 & 8 & .8 & 1.2 & \text { SNR } \\ 4.02 & 1.7 & 2.4 & 24 & 0 & 59 & .12 & 6 & .7 & 1.1 & \text { UER }\end{array}$ $\begin{array}{llllllllll}8.09 & 2.8 & 3.2 & 30 & 0 & 55 & .14 & 10 & .8 & 1.2\end{array}$ UKF

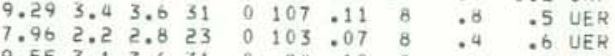
9.553 .13 .631 0 $84: 10 \quad 9 \quad: 6 \quad: 3$ UER $\begin{array}{lllllllllllll}8.80 & 3.0 & 3.4 & 31 & 0 & 99 & .10 & 7 & 9 & 7 & .5 & 5\end{array}$ $\begin{array}{rrrrrrrrrr}7.22 & 2.2 & 2.7 & 27 & 0 & 78 & .14 & 6 & .8 & 1.1 \\ 9.45 & 3 & \text { UER }\end{array}$ \begin{tabular}{rrrrrrrrrr}
9.45 & 3.6 & 4.1 & 30 & 0 & 112 & .11 & 7 & .8 & .5 \\
7.46 & 3.5 & 3.9 & 30 & 0 & 84 & .10 & 9 & .5 & .5 \\
\hline
\end{tabular}

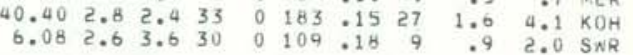

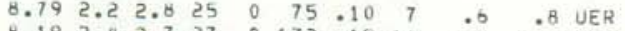

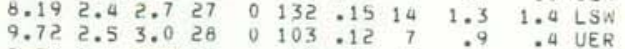

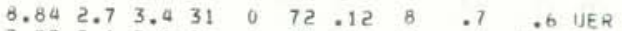

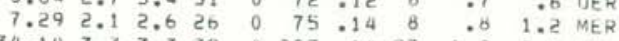

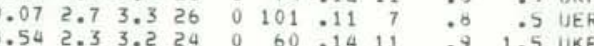

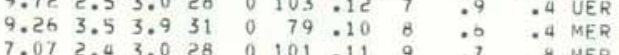


hVO EARTHGUAKE SUMMARY LIST

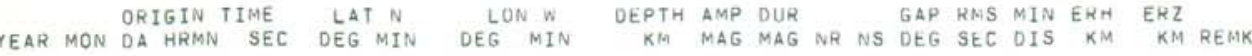

1976 OCT $722914.101922 .00 \quad 155 \quad 6.20$

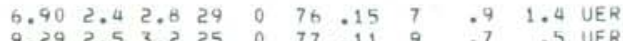
$8162929.8019 \quad 33.19$ 155 $51.58 \quad 26.50 \quad 3.3 \quad 3.3 \quad 37 \quad 2 \quad 147.18$ 15 $1.3 \quad 2.8 \mathrm{KON}$

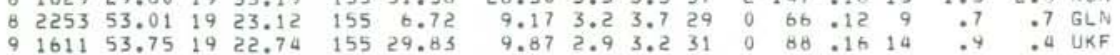
$\begin{array}{llllllll}10 & 5 & 1 & 26.28 & 19 & 30.12 \quad 155 & 15.57\end{array}$

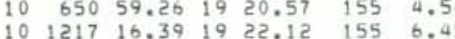
$\begin{array}{rrrrrrr}10 & 13 & 55.84 & 49 & 21.56 & 155 & 8.13 \\ 10 & 1740 & 38.75 & 19 & 19.56 & 155 & 11.03\end{array}$ $\begin{array}{llllll}10 & 1931 & 50.85 & 19 & 24.02 \quad 155 \quad 27.76\end{array}$

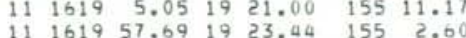
$11 \quad 1621 \quad 5.02 \quad 1923.43 \quad 155 \quad 2.00$

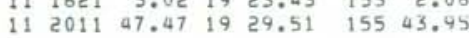

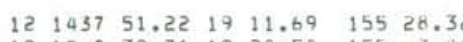

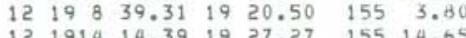
12 12 $191414.391927 .27 \quad 15514.65$ $1318 \quad 8 \quad 2.45 \quad 1920.30 \quad 155 \quad 16.46$

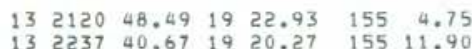
$13 \quad 2328 \quad 39.9919 \quad 17.04 \quad 155 \quad 23.32$ $\begin{array}{lllllll}14 & 819 & 48.75 & 19 & 26.51 & 154 & 55.23 \\ 14 & 950 & 49.25 & 19 & 15.19 & 155 & 18.97\end{array}$

$\begin{array}{lllllll}14 & 1211 & 17.59 & 19 & 19.35 \quad 155 & 12.5\end{array}$ $\begin{array}{lllllll}14 & 1336 & 20.13 & 19 & 25.24 & 155 & 17.17 \\ 14 & 1417 & 9.59 & 19 & 22.19 & 155 & 5.00\end{array}$ $14 \quad 1815 \quad 56.201927 .54 \quad 15521.27$

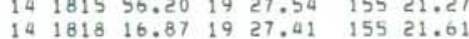

$\begin{array}{lrrrrrr}14 & 2317 & 6.95 & 19 & 22.67 & 155 & 5.12\end{array}$

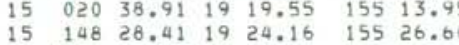

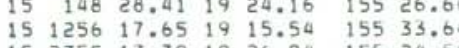
15235513.791926 .94 155 24.56

$\begin{array}{lllllll}16 & 1921 & 20.53 & 19 & 20.28 & 155 & 9.75\end{array}$ $17 \quad 557 \quad 16.7519 \quad 20.04 \quad 1558.27$

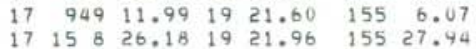

$\begin{array}{lllllll}17 & 2242 & 15.07 & 19 & 22.09 & 155 & 5.00\end{array}$ $172245 \quad 7.101920 .70 \quad 155 \quad 4.25$

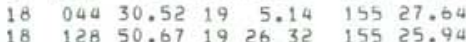
is $13350.081923 .87 \quad 15526.87$

$\begin{array}{lllllll}18 & 739 & 15.52 & 19 & 26.63 & 154 & 56.55\end{array}$ $\begin{array}{rrrrrrr}18 & 1754 & 8.90 & 19 & 19.62 & 155 & 11.69 \\ 18 & 1932 & 50.31 & 19 & 37.23 & 155 & 48.93\end{array}$ $\begin{array}{lllllllllll}9.40 & 2.2 & 2.7 & 31 & 0 & 101 & .15 & 13 & .7 & 1.1 & \text { GLN }\end{array}$

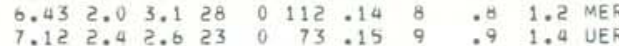

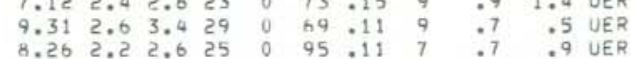
\begin{tabular}{llllllllll}
9.21 & 2.5 & 3.0 & 32 & 0 & 44 & .17 & 13 & .8 & 1.4 \\
\hline
\end{tabular} $\begin{array}{rrrrrrrrrr}9.61 & 2.2 & 2.6 & 24 & 0 & 76 & .11 & 8 & .8 & .5 \\ 7.78 & 2.2 & 2.5 & 23 & 0 & 127 & .12 & 11 & .8 & 1.0 \\ 9.0 & \text { MER }\end{array}$ $\begin{array}{lllllllllll}8.71 & 1.2 & 9 & 0 & 136.06 & 10 & .8 & 1.8 & \text { MER }\end{array}$

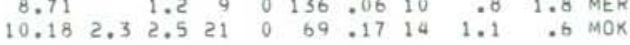
$\begin{array}{lllllllllllll}6.91 & 2.2 & 2.7 & 20 & 0 & 112 & .19 & 16 & 1.4 & 3.2 & \text { LSW }\end{array}$

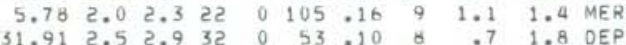

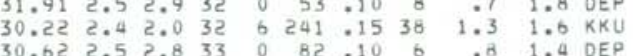
$\begin{array}{lllllllllll}7.61 & 2.4 & 2.7 & 24 & 0 & 89 & .10 & 9 & .5 & .8 & \text { MER } \\ 9.04 & 1.7 & 2.5 & 26 & 0 & 77 & : 13 & 7 & .7 & : 9 & \text { UER }\end{array}$

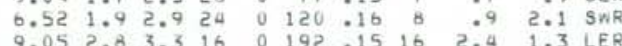

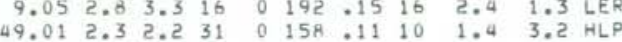
$\begin{array}{llllllllll}7.63 & 2.0 & 2.7 & 23 & 0 & 86 & .13 & 7 & .8 & 1.4 \\ 9.4 & \text { UER }\end{array}$

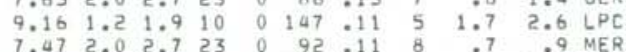

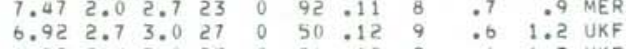

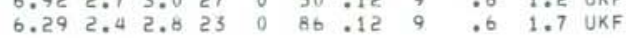

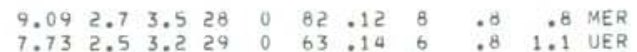

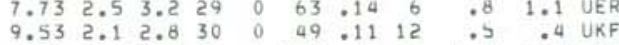

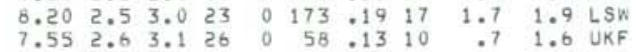

$\begin{array}{lllllllllll}9.11 & 2.1 & 2.3 & 23 & 0 & 78 & .08 & 7 & .5 & .6 & \text { UER }\end{array}$

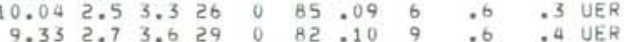

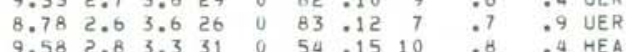
$7.962 .02 .624 \quad 0 \quad 79.08$ \& 2.5 . B MER

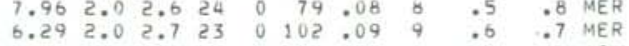

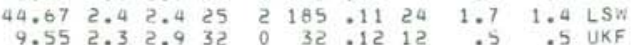

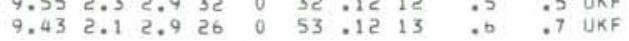

$\begin{array}{llllllllll}4.92 & 2.4 & 2.9 & 26 & 4 & 172 & .20 & 14 & 1.3 & 1.4 \\ 7.39 & \text { LER }\end{array}$

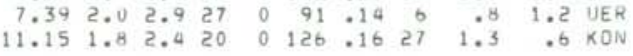

HVO EARTHQUAKE SUMMARY LIST

PAGE 40 DRIGIN TIME LAT N LON W DEPTH AMP DUR GAP RMS MIN ERH ERZ YEAR MON DA HRMN SEC DEG MIN DEG MIN KM MAG MAG NR NS DEG SEC OIS KM KM REMK

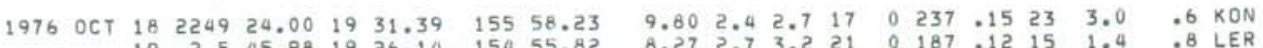

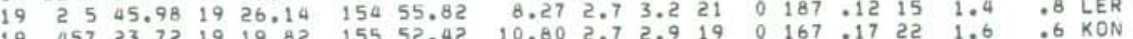

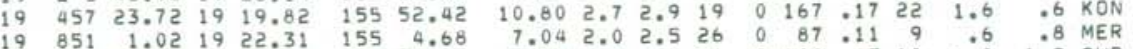

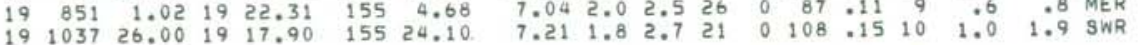
$\begin{array}{llllllllllllllllllll}19 & 2311 & 50.41 & 19 & 20.31 & 155 & 6.99 & 9.03 & 2.7 & 3.6 & 28 & 0 & 101 & .11 & 7 & .7 & .6 & \text { UER }\end{array}$

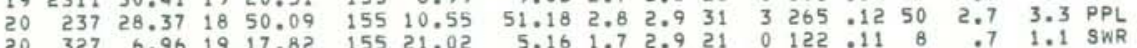

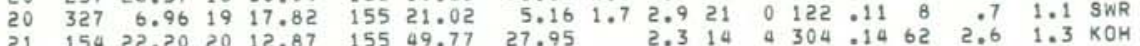

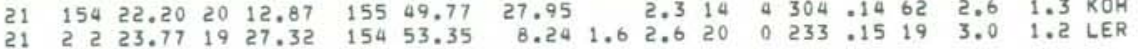

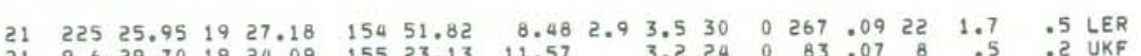

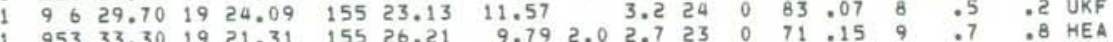

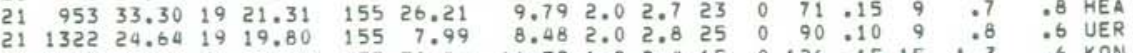

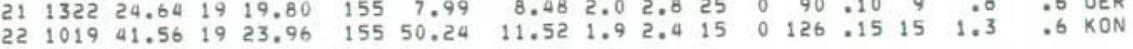

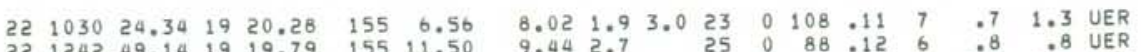

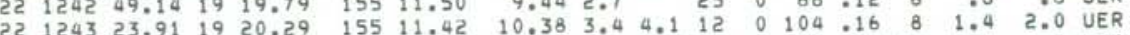

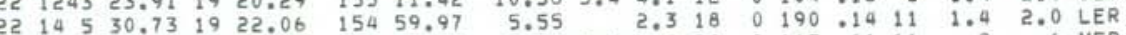
$22 \quad 1411 \quad 25.90 \quad 19 \quad 21.20 \quad 155 \quad 3.33$ $\begin{array}{llllllll}22 & 16 & 0 & 15.12 & 19 & 19.26 & 155 & 11.75\end{array}$ $\begin{array}{rrrrrrr}22 & 1624 & 40.51 & 19 & 20.04 & 155 & 11.53 \\ 23 & 919 & 6.64 & 19 & 20.09 & 155 & 5.31\end{array}$

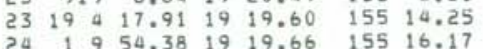
$24 \quad 2 \quad 0 \quad 53.33 \quad 19 \quad 28.18 \quad 154 \quad 54.39$ $24 \quad 519 \quad 46.49 \quad 1920.02 \quad 155 \quad 8.19$ $24 \quad 818 \quad 53.98$ 19 $22.14 \quad 155 \quad 6.34$ $24 \quad 833 \quad 54.79 \quad 1922.29 \quad 154 \quad 59.87$

$\begin{array}{lllllll}24 & 1245 & 27.08 & 19 & 18.83 & 155 & 22.31\end{array}$ 24 $184123.82 \quad 1927.04 \quad 15514.96$ 24 201026.461921 .191557 .83 $25 \quad 247 \quad 25,47 \quad 19 \quad 10.61 \quad 155 \quad 33.34$

$\begin{array}{lllllll}25 & 525 & 11.76 & 19 & 19.82 & 155 & 11.81 \\ 25 & 526 & 1.86 & 19 & 21.00 & 155 & 8.48\end{array}$

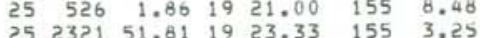
25232151.811923 .331553 .25

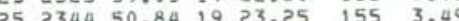
$\begin{array}{lllllll}26 & 657 & 44.92 & 19 & 20.38 & 155 & 8.83 \\ 26 & 7 & 75.35 & 19 & 21.74 & 155 & 15.18\end{array}$

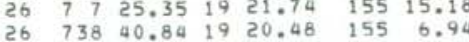
$\begin{array}{lllllll}26 & 738 & 40.84 & 19 & 20.74 & 155 & 15.10 \\ 26 & 754 & 41.58 & 19 & 19.73 & 155 & 8.2\end{array}$ $26 \quad 83917.0019$ 19.75 1550.31

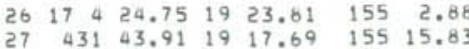

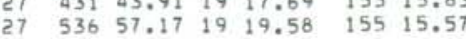

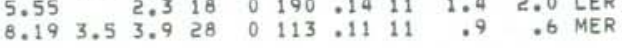
$\begin{array}{llllllllllll}7.30 & 2.0 & 2.6 & 28 & 0 & 98 & .12 & 7 & .7 & 1.1 & \text { UER }\end{array}$

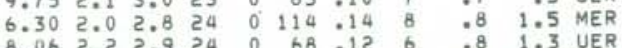

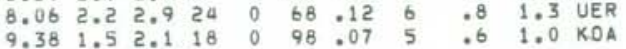
\begin{tabular}{lllllllllll}
8.15 & 1.8 & 2.3 & 18 & 2 & 172 & .21 & 17 & 1.9 & 1.4 & LER \\
\hline & 9.9 & 5 & UER
\end{tabular} \begin{tabular}{llllllllll}
5.64 & 2.5 & 3.4 & 27 & 0 & 185 & .13 & 11 & 1.1 & 1.4 \\
5.10 & & 2.2 & 14 & 0 & 188 & .12 & 10 & 1.6 & 1.1 \\
\hline & LER
\end{tabular} $\begin{array}{llllllllllll}7.74 & 2.3 & 3.1 & 24 & 0 & 105 & .15 & 9 & .9 & 1.8 & \text { SWR }\end{array}$

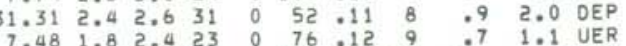

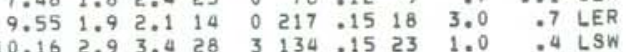

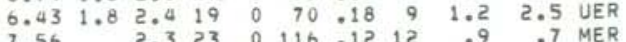

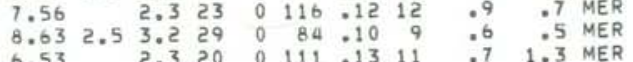
$\begin{array}{llllllllllllll}8.52 & 2.0 & 2.8 & 24 & 0 & 138 & .09 & 10 & .6 & .7 & \text { UER }\end{array}$

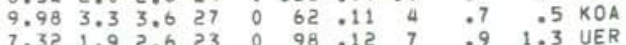

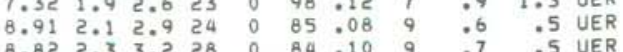
$\begin{array}{llllllllllllll}8.45 & 2.5 & 2.2 & 16 & 0 & 120 & .10 & 11 & .8 & 1.0 & \text { MER }\end{array}$

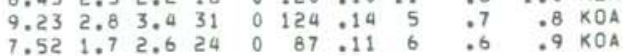

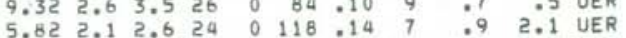

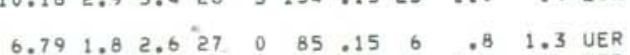


$\begin{array}{lllll}\text { ORIGIN TIME } & \text { LAT N LON N DEPTH AMP DUR } \\ \text { DA HRMN SEC OEG MIN DEG RMS MIN ERH ERZ }\end{array}$

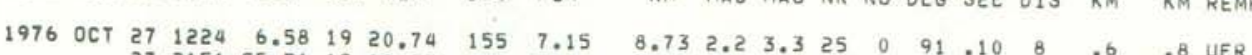

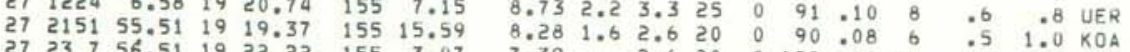

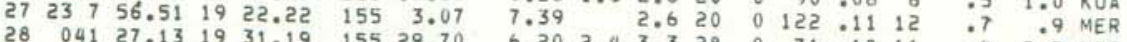

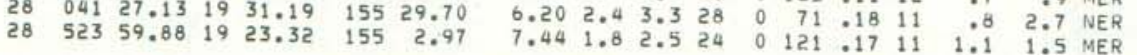

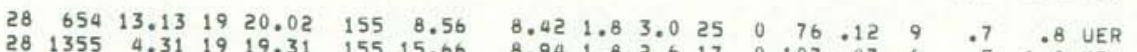

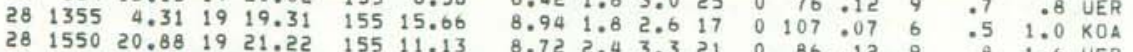

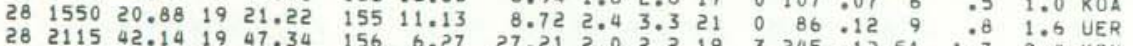

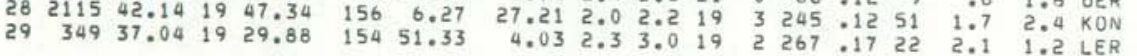

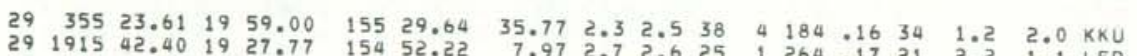

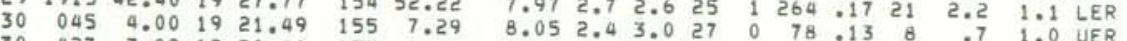

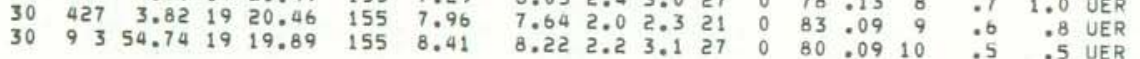

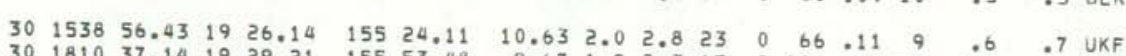

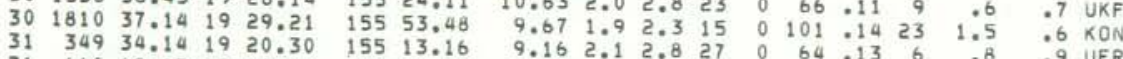

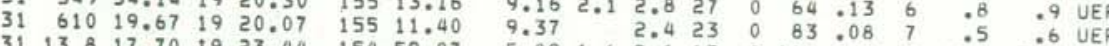

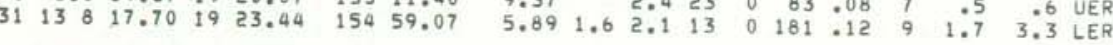

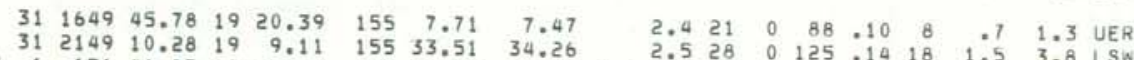
NoV 1 151 $46.87 \quad 1928.31 \quad 15522.82$ $14421.531918 .76 \quad 15529.13$

$1746 \quad 10.02 \quad 1929.77 \quad 15453.55$ $1813 \quad 50.7919$
19 $\begin{array}{lllllllll}1 & 10 & 1 & 23.83 & 19 & 21.14 & 155 & 6.69\end{array}$

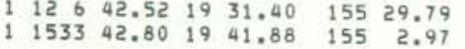
$\begin{array}{lllllll}1 & 1617 & 56.56 & 19 & 26.02 & 155 & 24.73\end{array}$ $1756 \quad 55.35$
2

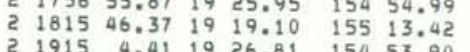
$\begin{array}{llllll}1915 & 4.41 & 19 & 26.81 & 154 & 53.90\end{array}$ $\begin{array}{lrrrrrr}2 & 1936 & 16.05 & 19 & 22.50 & 155 & 5.86 \\ 2 & 2230 & 6.49 & 19 & 19.48 & 155 & 15.96\end{array}$

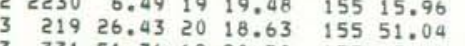
$\begin{array}{lllllll}3 & 331 & 51.36 & 19 & 20.70 & 155 & 5.81\end{array}$ \begin{tabular}{l}
$740 \quad 57.15 \quad 19 \quad 20.83 \quad 155 \quad 6.91$ \\
\hline
\end{tabular} $\begin{array}{lllllll}3 & 740 & 57.15 & 19 & 20.83 & 155 & 6.91 \\ 3 & 755 & 23.35 & 19 & 20.87 & 155 & 9.70\end{array}$

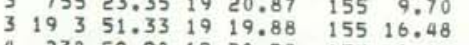
$\begin{array}{lllllll}4 & 238 & 59.98 & 19 & 20.78 & 155 & 6.19\end{array}$

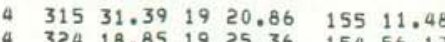
$\begin{array}{lllllll}4 & 324 & 18.85 & 19 & 25.36 & 154 & 56.12 \\ 4 & 358 & 26.17 & 19 & 19.31 & 155 & 11.56\end{array}$

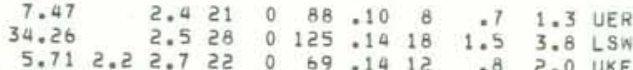

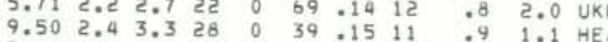

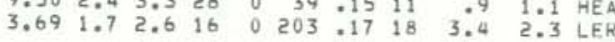
$\begin{array}{lllllllllllll}3.69 & 1.7 & 2.6 & 16 & 0 & 203 & .17 & 18 & 3.4 & 2.3 & \text { LER }\end{array}$

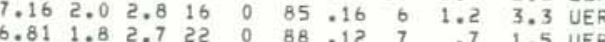
$8.382 .333 .022 \quad 0 \quad 180.12 \quad 7 \quad 071.5$ UER $\begin{array}{llllllllllll}8.30 & 1.7 & 2.7 & 24 & 0 & 58 & .16 & 10 & .9 & 1.9 & \text { UKF }\end{array}$

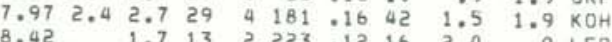
$\begin{array}{rrrrrrrrrrr}8.42 & 1.7 & 13 & 2 & 223 & 12 & 16 & 2.0 & .9 & \text { LER } \\ 0.12 & 3.7 & 4.0 & 29 & 0 & 73 & .08 & 7 & .5 & .9 & .5 \text { UER }\end{array}$ $\begin{array}{lllllllll}.95 & 1.5 & 13 & 0 & 230 & .17 & 18 & 3.8 & .9\end{array}$ $\begin{array}{rrrrrrrrrrr}8.74 & 3.3 & 3.7 & 29 & 0 & 71 & .12 & 7 & .7 & .7 & \text { MER } \\ 8.42 & 1.7 & 2.6 & 24 & 0 & 92 & .11 & 5 & .7 & 1.0 & \text { KOA }\end{array}$

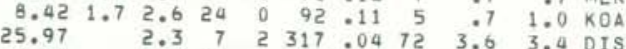

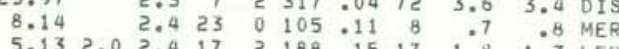
$\begin{array}{llllllllll}5.98 & 2.4 & 21 & 0 & 92 & 15 & 7 & 1.0 & 2.0 & \text { UER }\end{array}$

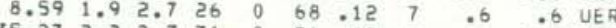

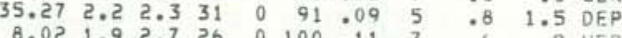
$\begin{array}{lllllllllll}7.42 & 1.9 & 2.7 & 26 & 0 & 100 & .11 & 7 & .6 & .9 & 9 \text { UER } \\ 7.42 & 2.0 & 2.6 & 27 & 0 & 95 & .14 & 11 & .8 & 1.5 & \text { UKF }\end{array}$ $\begin{array}{lllllllllll}7.00 & 1.8 & 2.6 & 13 & 0 & 79 & .09 & 9 & .9 & 1.8 & \text { UER }\end{array}$

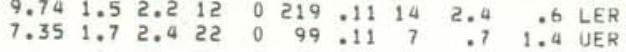
$1.152 .02 .810 \quad 0248.14 \quad 32 \quad 3.853 .9$ NEF
YEAR MON ORIGIN TIME LAT N LON SEC DEG MIN DEPTH AMP DUR GAP RMS MIN ERH ERZ

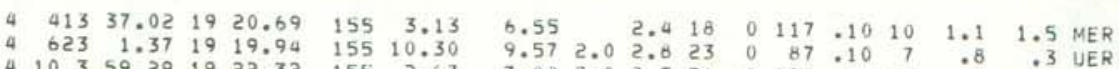

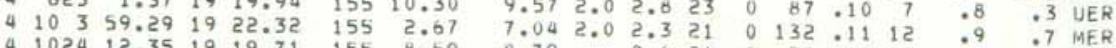

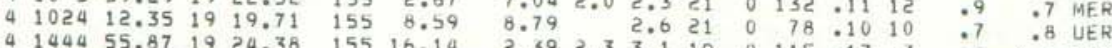

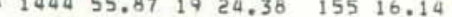

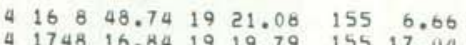
4224854.881919 .79 155 17.04

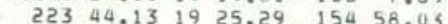
$\begin{array}{llllll}5 & 258 & 29.68 & 19 & 21.38 & 155 \quad 8.19\end{array}$

$\begin{array}{llllllll}5 & 621 & 14.13 & 19 & 15.31 & 155 & 22.90\end{array}$ $\begin{array}{lllllll}5 & 1134 & 27.13 & 19 & 19.41 & 155 & 15.52\end{array}$ $51347 \quad 48.531921 .94 \quad 155 \quad 6.98$ $5 \quad 1446 \quad 58.16 \quad 19 \quad 19.91 \quad 155 \quad 11.7$

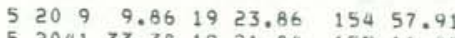

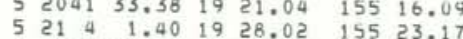
$6 \quad 613 \quad 36.76 \quad 1922.20 \quad 15525.36$ $6211 \quad 14.621931 .39 \quad 15529.90$
0

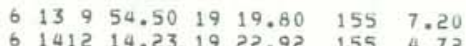

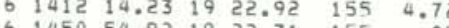
$1738 \quad 37.07$
6 $1928.01 \quad 15452.20$

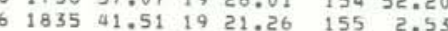

$\begin{array}{lllllll}6 & 2340 & 47.26 & 19 & 23.58 & 155 & 25.04\end{array}$

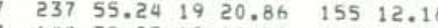

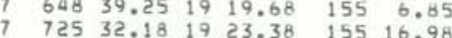
$727 \quad 30.26 \quad 1924.16 \quad 155 \quad 16.02$

$\begin{array}{lllllll}7 & 1050 & 25.98 & 19 & 19.50 & 155 & 12.23\end{array}$ $\begin{array}{lllllll}11 & 1 & 13.83 & 19 & 22.08 & 154 & 58.21\end{array}$ $7153637.6919 \quad 19.45 \quad 15511.66$ $\begin{array}{lllllll}7 & 1623 & .27 & 19 & 20.11 & 155 & 18.82 \\ 7 & 1854 & 56.85 & 19 & 15.54 & 155 & 34.53\end{array}$

$\begin{array}{lllllll}8 & 015 & 24.88 & 19 & 19.78 & 155 & 7.64\end{array}$ $\begin{array}{lllllll}8 & 434 & 7.94 & 19 & 20.49 & 155 & 11.11\end{array}$ $\begin{array}{lrrrrr}645 & 46.31 & 19 & 20.05 & 155 & 13.18\end{array}$

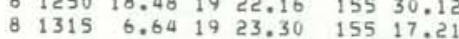

$\begin{array}{lllllll}8 & 1818 & 20.39 & 19 & 18.93 \quad 155 & 13.55\end{array}$ $81916 \quad 18.84$ i $\begin{array}{lllllll}8 & 1951 & 5.80 & 1923.36 & 155 & 4.35\end{array}$ $9040 \quad 2.31 \quad 1926.05 \quad 154 \quad 56.15$ $\begin{array}{llll}516 & 25.14 & 19 & 22.71 \quad 155 \quad 4.62\end{array}$

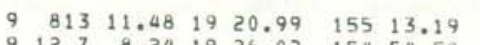
$9141944.591919 .08 \quad 15513.92$

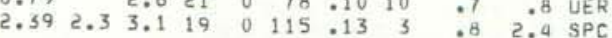

$\begin{array}{llllllllllll}7.89 & 3.0 & 3.1 & 27 & 0 & 90 & .13 & 7 & .8 & .8 & \text { UER }\end{array}$ $\begin{array}{lllllllllll}8.21 & 1.5 & .5 & 17 & 0 & 90 & .13 & 5 & 1.1 & 1.7 & \text { KOA } \\ 8.82 & 2.4 & 3.1 & 25 & 0 & 97 & 0.11 & 8 & .7 & .6 & \text { UER }\end{array}$

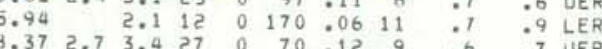
$\begin{array}{llllllllll}3.44 & 2.7 & 24 & 0 & 137 & .14 & 9 & .9 & 1.7 & \text { LS }\end{array}$

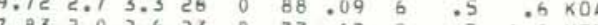

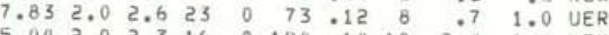

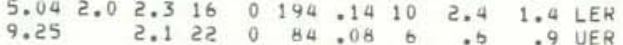

$\begin{array}{llllllllll}7.70 & 2.4 & 17 & 0 & 190 & .15 & 11 & 2.0 & 1.4 & \text { LER }\end{array}$ $\begin{array}{lllllllllll}74.12 & 2.7 & 2.8 & 32 & 0 & 70 & .09 & 5 & .7 & 1.6 & \text { DEP }\end{array}$

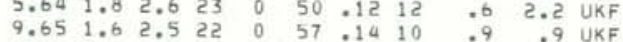
$5.612 .52 .824 \quad 0 \quad 101: 16 \quad 11$ :8 8.9 NKF

\begin{tabular}{lllllllllll}
8.34 & 2.0 & 2.4 & 26 & 0 & 108 & .12 & 8 & .7 & .8 & .8 \\
\hline UER
\end{tabular}

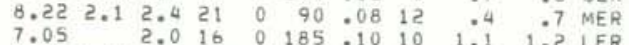

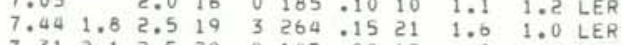

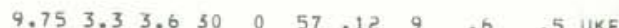

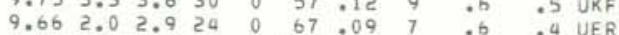
$\begin{array}{llllllllll}8.71 & 2.1 & 2.8 & 25 & 0 & 117 & .09 & 7 & .6 & .5 \\ 1.5 & 1.0 & 2.1 & 10 & 0 & 63 & .10 & 3 & .0 & .3\end{array}$

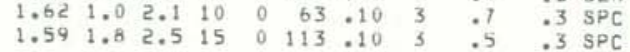

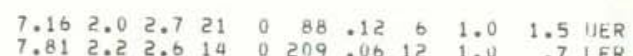

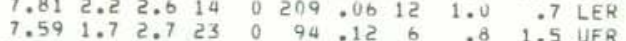

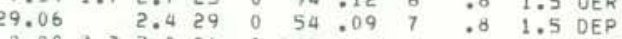
$\begin{array}{lllllll} & 0.7 & 169 & .20 & 18 & 1.6 & 2.7 \\ \text { HEA }\end{array}$ $\begin{array}{lllllllllll}7.38 & 1.9 & 2.8 & 25 & 0 & 98 & .13 & 8 & .8 & 1.5 \\ 9.18 & 1.7 & 2.6 & 22 & 0 & 77 & .13 & 8 & .8 & \end{array}$

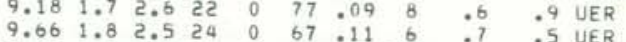

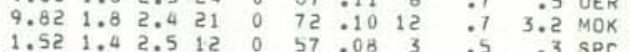
$\begin{array}{lllllllllllll}9.65 & 1.7 & 2.7 & 22 & 0 & 72 & .11 & 7 & .7 & .7 & \text { POL }\end{array}$ $\begin{array}{llllllllll}7.54 & 1.6 & 2.5 & 17 & 0 & 96 & .10 & 10 & .7 & 1.5 \\ 7.92 & 2.9 & 3.7 & 29 & 0 & 70 & .10 & 7 & .5 & \\ 7.25 R\end{array}$

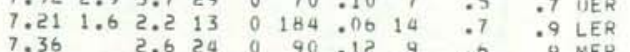

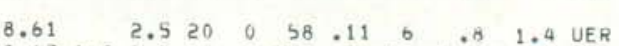

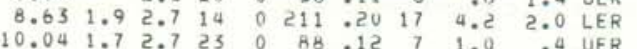




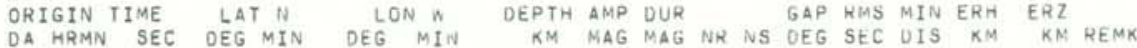
1976 NOV $9 \begin{array}{llllllllllllllllll}1533 & 3.39 & 19 & 20.60 & 155 & 4.14 & 8.06 & 3.8 & 4.1 & 32 & 0 & 107 & .12 & 9 & .9 & .6 & .6 & \text { MER }\end{array}$

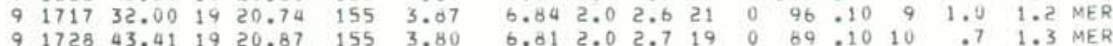

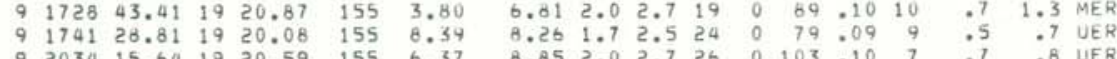

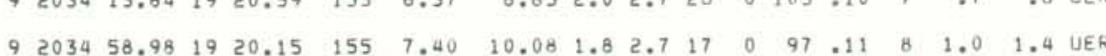

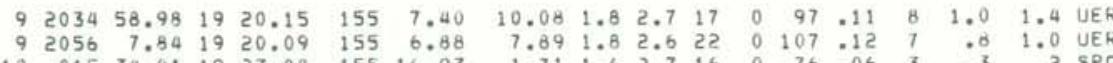

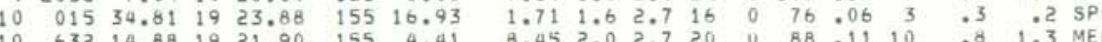

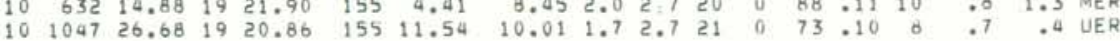

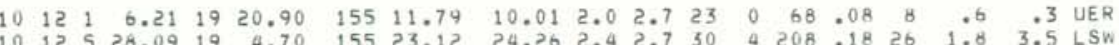

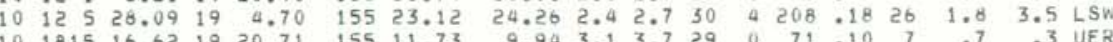

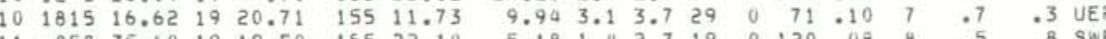

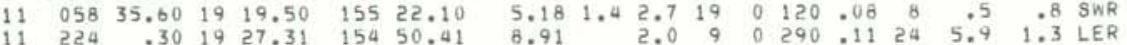

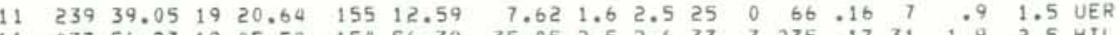

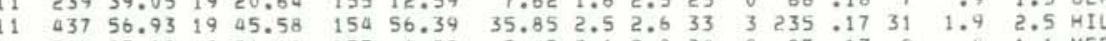

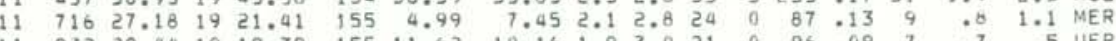

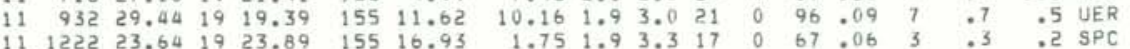
$11 \quad 1236 \quad 10.711921 .09 \quad 155 \quad 3.53$

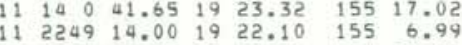
$.66 \quad 154 \quad 55.37$

$12 \quad 22018.69 \quad 1925.75 \quad 15454.64$ 12 टट2 $44.231927 .56 \quad 15450.71$ $12 \quad 319 \quad 12.691927 .28 \quad 155 \quad 26.81$

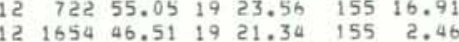

1220721.451956 .64015536 .00

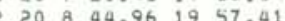
$\begin{array}{lllllll}13 & 918 & 49.51 & 19 & 13.99 & 155 & 17.17\end{array}$

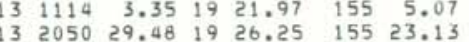

$14 \quad 419 \quad 23.06 \quad 19 \quad 25.52 \quad 155 \quad 16.52$

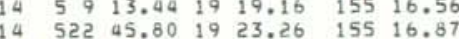
$\begin{array}{lllllll}14 & 120 & 51.05 & 19 & 21.30 & 155 & 2.32\end{array}$

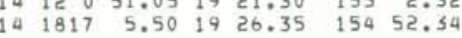

$14 \quad 2040 \quad 36.96 \quad 19 \quad 20.18 \quad 155 \quad 8.52$ $142325 \quad 14.40 \quad 1923.66 \quad 155 \quad 16.89$

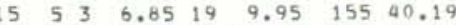
$\begin{array}{lllllll}15 & 917 & 7.99 & 19 & 20.80 & 155 & 13.02\end{array}$ $15 \quad 1035 \quad 59.09 \quad 1921.07 \quad 155 \quad 8.43$

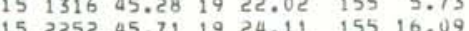
$\begin{array}{lllllllllll}0.36 & 2.7 & 3.2 & 28 & 0 & 70 & .11 & 9 & .0 & .7 & .7 \\ 7.59 R\end{array}$

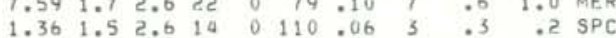

ORIGIN TIME LAT N LON W
DA HRMN SEC DEG MIN DEF MIN

1976 NOV $16 \quad 040 \quad 34.83 \quad 19 \quad 24.14 \quad 155 \quad 16.14$ $\begin{array}{lllllll}16 & 056 & 29.30 & 19 & 22.38 & 155 & 4.87 \\ 16 & 223 & 35.43 & 19 & 22.59 & 155 & 4.70\end{array}$

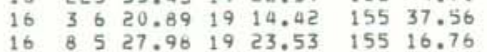
$\begin{array}{llllll}16 & 1123 \quad 22.09 \quad 19 \quad 23.38 \quad 155 \quad 16.98\end{array}$ $\begin{array}{llllllll}6 & 12 & 3 & 48.16 & 19 & 17.80 & 155 & 16.22\end{array}$

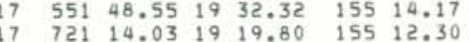
$\begin{array}{lllllll}17 & 1213 & 8.59 & 19 & 23.05 \quad 155 \quad 16.84\end{array}$ $\begin{array}{lllllll}17 & 1232 & 43.66 & 19 & 20.45 & 155 & 13.62\end{array}$ $18 \quad 3458.48 \quad 1920.56 \quad 15519.33$ $\begin{array}{llllllll}18 & 3 & 720.46 & 19 & 26.87 & 155 & 23.60\end{array}$

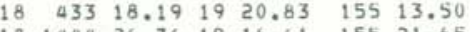
$\begin{array}{llllllll}18 & 1444 & 26.76 & 19 & 16.61 & 155 & 21.65\end{array}$ $18 \quad 155541.88 \quad 1923.66 \quad 15458.15$

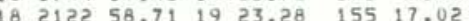

$\begin{array}{lllllll}18 & 2224 & 43.77 & 19 & 23.87 & 155 & 16.89\end{array}$ $\begin{array}{llrllll}19 & 318 & 8.70 & 18 & 59.10 & 155 & 22.58 \\ 19 & 352 & 14.10 & 19 & 23.55 & 154 & 58.13\end{array}$ $19 \quad 352 \quad 14.101923 .55 \quad 15458.13$ $19 \quad 1613 \quad 52.84 \quad 19 \quad 18.21 \quad 155 \quad 16.84$

$192254 \quad 37.19 \quad 1920.11 \quad 155 \quad 13.47$ $\begin{array}{lllllll}20 & 711 & 1.33 & 19 & 23.34 & 155 & 17.07 \\ 20 & 23 & 37.02 & 19 & 24.10 & 155 & 15.72\end{array}$ दि 238827.021924 .100 155 15.72 ट1 $25753.321919 .98 \quad 155 \quad 7.76$

$\begin{array}{lllllll}21 & 2035 & 13.51 & 19 & 22.23 & 155 & 6.72\end{array}$ $21 \quad 2046 \quad 44.06 \quad 1923.03 \quad 155 \quad 4.79$

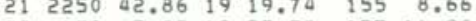

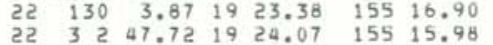

$\begin{array}{lllllll}22 & 513 & 50.38 & 19 & 20.56 & 155 & 7.44\end{array}$

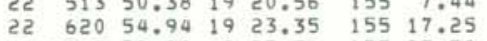
$2210532.00 \quad 1925.56 \quad 155 \quad 23.50$

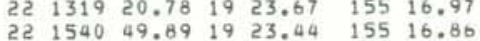
$\begin{array}{lll}10 & 155 \quad 6.29\end{array}$ $23 \quad 332 \quad 47.70 \quad 1922.76 \quad 155 \quad 4.86$

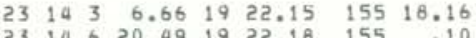
$23 \quad 1412 \quad 14.97 \quad 20 \quad 10.09 \quad 155 \quad 50.10$ $\begin{array}{lllllll}22 & 1831 & 19.81 & 19 & 19.86 & 155 \quad 7.12\end{array}$

DEPTH AMP DUR
KM MAG MAG NR NS DEP RMS SEC DIS KM KM KM REMK $\begin{array}{lllllllllll}1.61 & 1.5 & 2.7 & 12 & 0 & 119 & .07 & 3 & .5 & .3 & \text { SPC }\end{array}$ $9.203 .6 \quad 4.1 \quad 32 \quad 0 \quad 88.12 \quad 9 \quad 08$

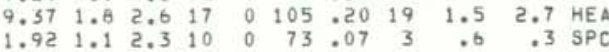
$\begin{array}{llllllllllll}1.89 & 2.1 & 3.0 & 13 & 0 & 63 & .07 & 3 & .5 & .3 & \mathrm{SPC}\end{array}$ $\begin{array}{lllllllllll}.09 & 1.7 & 2.9 & 17 & 0 & 127 & .11 & 5 & .8 & 1.7 & \mathrm{KO}\end{array}$

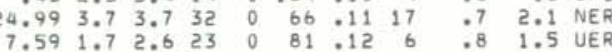
$\begin{array}{lllllllllll}1.96 & 2.1 & 3.0 & 16 & 0 & 53 & .14 & 3 & .8 & .5 & \text { SPC }\end{array}$

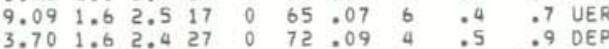
$\begin{array}{llllllllll}8.78 & 1.6 & 2.5 & 21 & 0 & 57.10 & 7 & .7 & 1.9 & \text { SWP }\end{array}$ $\begin{array}{llllllllllllllll}8.27 & 2.0 & 2.7 & 21 & 0 & 74 & .11 & 11 & .7 & 1.3 & \text { UKF }\end{array}$ $\begin{array}{lllllllllll}9.65 & 2.3 & 3.1 & 27 & 0 & 56 & -10 & 7 & .6 & .4 & \text { UER }\end{array}$

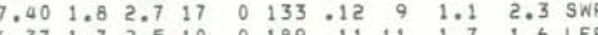

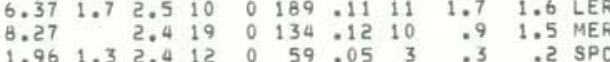
$\begin{array}{rrrrrrrrrrr}3.79 & 3.0 & 3.8 & 24 & 0 & 69 & .13 & 3 & .7 & 1.0 & \mathrm{SPC} \\ 31.13 & 2.9 & 2.9 & 31 & 0 & 227 & .10 & 33 & 1.8 & 3.2 & \mathrm{DIS}\end{array}$ $\begin{array}{lllllllll}4.97 & 2.4 & 12 & 0 & 191 & 11 & 11 & 1.6 & 1.2 \\ 0 & \text { LER }\end{array}$

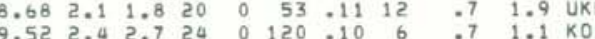

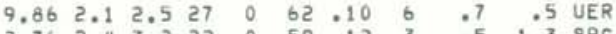

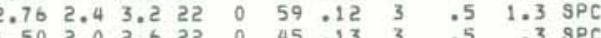

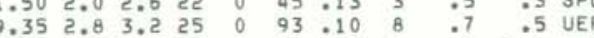

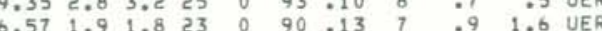

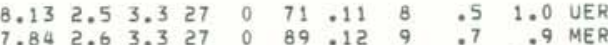

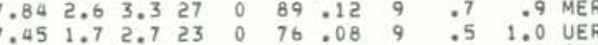

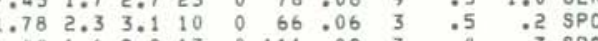

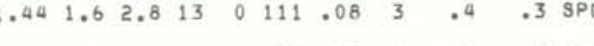

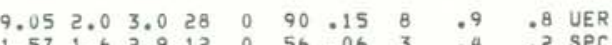

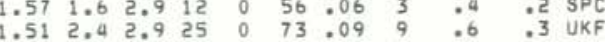
$\begin{array}{lllllllllll}2.00 & 1.8 & 2.8 & 13 & 0 & 70 & .06 & 3 & .4 & 1.1 & \text { SPC }\end{array}$

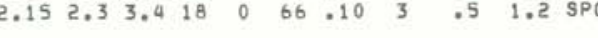

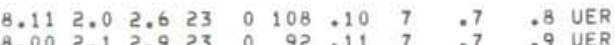
$\begin{array}{lllllllllll}0.03 & 2.4 & 3.1 & 17 & 0 & 90 & -16 & 20 & 1.2 & .6 & \text { KON }\end{array}$

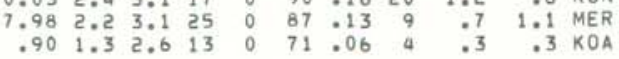
$\begin{array}{llllllrlllll}1.22 & 1.6 & 2.7 & 10 & 0 & 70 & .03 & 4 & .1 & .1 & \text { KOA }\end{array}$ 
HVO EARTHQUAKE SUMMARY LIST

PAGE 45 ORIGIN TIME LAT N LON W DEPTH AMP DUR GAP RMS MIN ERH ERZ YEAR MON DA HRMN SEC DEG MIN DEG MIN KM MAG MAG NR NS DEG SEC OIS KM KM REMK 1976 NOV $23 \quad 1541 \quad 47.61 \quad 1925.99 \quad 15453.66$

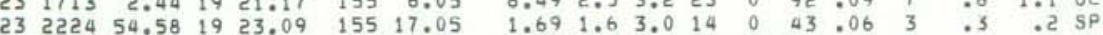

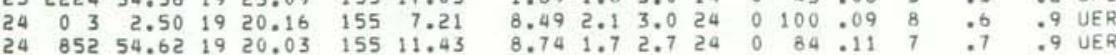
$\begin{array}{llllllllllllllllll}24 & 1157 & 37.56 & 19 & 20.94 & 155 & 6.25 & 8.39 & 2.2 & 3.2 & 23 & 0 & 96 & .11 & 7 & .8 & .8 & \text { UER }\end{array}$

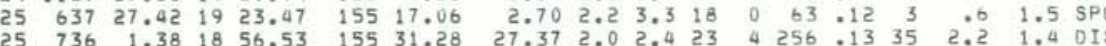
$25 \quad 843 \quad 33.53 \quad 1921.79 \quad 15515.34$

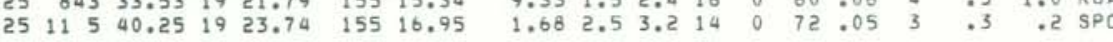

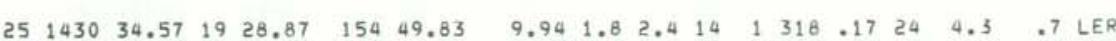

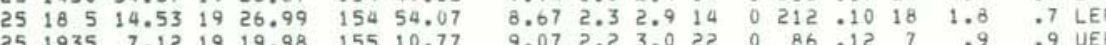

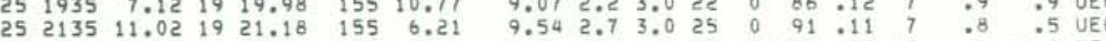

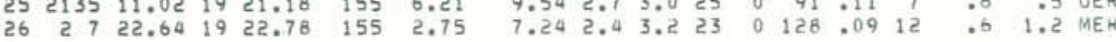

$26 \quad 349 \quad 14.70 \quad 19 \quad 24.25 \quad 155 \quad 16.07$ $26 \quad 635 \quad 59.15 \quad 1920.96 \quad 155 \quad 13.06$ 26 1015 58.54 1924.87 $155 \quad .80$ $26 \quad 1317 \quad 4.61 \quad 19 \quad 19.19 \quad 155 \quad 13.78$

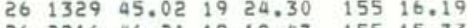

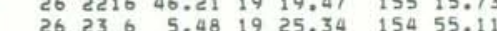
$27 \quad 335 \quad 34.051920 .41 \quad 15511.58$

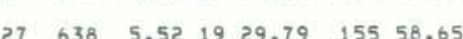
$\begin{array}{rrrrrrr}27 & 638 & 5.52 & 19 & 29.79 & 155 & 58.65 \\ 27 & 927 & 38.80 & 19 & 22.46 & 155 & 5.10\end{array}$ $27 \quad 1241 \quad 14.98 \quad 1925.42 \quad 155 \quad 25.45$ $27 \quad 1915 \quad 36.46 \quad 1923.89 \quad 155 \quad 16.88$

$\begin{array}{llllllll}28 & 256 & 42.01 & 19 & 23.37 & 155 & 16.91\end{array}$ $\begin{array}{lllllll}28 & 539 & 52.58 & 19 & 20.31 & 155 & 12.84\end{array}$ $\begin{array}{lllllll}28 & 1214 & 16.32 & 19 & 23.53 & 155 & 17.17\end{array}$ $28 \quad 2021 \quad 5.24 \quad 19 \quad 17.59 \quad 15521.53$

$\begin{array}{llllllll}29 & 5 & 1 & 27.55 & 19 & 21.16 & 155 & 6.42 \\ 29 & 7 & 6 & 30.61 & 19 & 18.85 & 155 & 13.58\end{array}$ $29 \quad 93 \quad 47.43 \quad 1927.09 \quad 15451.98$ $29103625.351921 .22 \quad 15513.4$

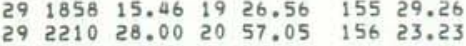
$\begin{array}{lllllll}30 & 120 & 37.03 & 19 & 19.28 & 155 & 13.87\end{array}$ $\begin{array}{lllllll}30 & 1746 & 10.06 & 19 & 19.46 & 155 & 11.39 \\ 30 & 1818 & 45.29 & 19 & 19.34 & 155 & 11.28\end{array}$

$\begin{array}{lllllll}30 & 1937 & 30.25 & 19 & 19.52 & 155 & 16.21\end{array}$

DEC $1 \quad 020 \quad 25.96 \quad 19 \quad 20.75 \quad 155 \quad 13.63$ $\begin{array}{lllllllllllll}1.61 & 2.1 & 3.2 & 18 & 0 & 114 & .07 & 3 & .3 & .2 & \text { SPC }\end{array}$

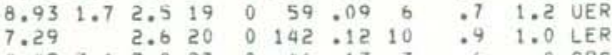

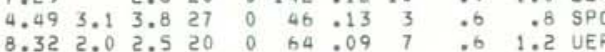

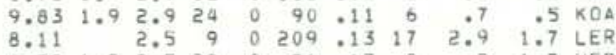
$\begin{array}{rrrrrrrrrrrr}8.58 & 1.7 & 2.5 & 22 & 0 & 76 & .13 & 8 & .9 & 1.7 & \text { UER } \\ 23.32 & 2.5 & 3.3 & 15 & 1 & 301 & .04 & 81 & 7.1 & 10.4 & \text { DIS }\end{array}$

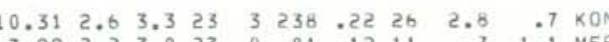

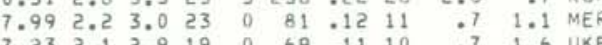
$\begin{array}{rrrrrrrrrrr}7.23 & 2.1 & 2.9 & 19 & 0 & 69 & .11 & 10 & .7 & 1.6 & \text { UKF } \\ 1.70 & 1.4 & 2.8 & 12 & 0 & 78 & .06 & 3 & .4 & .2 & \text { SPC } \\ 9.80 & 2.1 & 3.1 & & & & & & & & \end{array}$ $9.802 .13 .126 \quad 0 \quad 48.1112$.5 .4 UKF $\begin{array}{lllllllllll}2.02 & 1.6 & 2.9 & 18 & 0 & 64 & .10 & 3 & .5 & 1.3 & \text { SPC } \\ 8.01 & 1.6 & 2.8 & 24 & 0 & 68 & 111 & 7 & 7 & 1.1 & \text { UER }\end{array}$

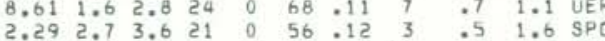
$\begin{array}{rrrrrrrrrrr}9.55 & 2.3 & 2.8 & 24 & 0 & 58 & .11 & 1.1 & .7 & .5 & \text { UKF } \\ 7.52 & 2.0 & 3.4 & 24 & 0 & 122 & .13 & 9 & .8 & 1.3 & \text { SWR }\end{array}$

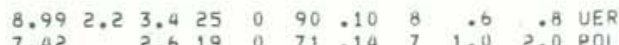

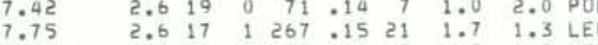
$\begin{array}{rrrrrrrrrrr}9.68 & 1.6 & 2.8 & 16 & 0 & 57 & .06 & 7 & .7 & 1.9 & \text { UER } \\ 8.21 & 2.5 & 13 & 0 & 316 & .10 & 23 & 5.4 & 1.0 & \text { LER }\end{array}$

$\begin{array}{lllllllllll}10.18 & 1.9 & 2.6 & 22 & 0 & 68 & .13 & 12 & .7 & .9 & \text { UKF }\end{array}$

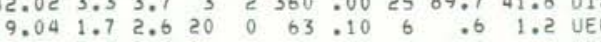

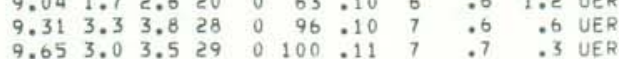
$\begin{array}{llllllllllll}9.60 & 2.5 & 3.4 & 32 & 0 & 94 & .11 & 5 & .6 & .7 & \text { KOA }\end{array}$

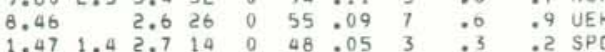
$\begin{array}{llllllllllll}1.73 & 1.4 & 2.6 & 12 & 0 & 115 & .05 & 3 & .4 & .2 & \text { SPC }\end{array}$
HVO EARTHQUAKE SUMMARY LIST

PAGE 46

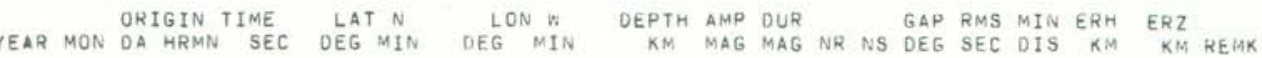

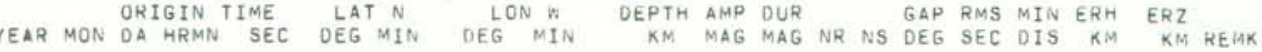

$\begin{array}{llllllll}1976 & \text { DEC } 1 & 444 & 17.47 & 19 & 21.64 & 155 & 15.23\end{array}$ 62259.481921 .6425515 .23 $1057 \quad 37.57 \quad 1920.35 \quad 155 \quad 13.42$
19

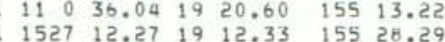

$\begin{array}{lllllll}2 & 015 & 33.29 & 19 & 23.63 & 155 & 17.18\end{array}$

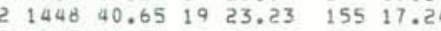
$1613 \quad 44.5419 \quad 22.06 \quad 155 \quad 6.75$
250

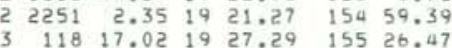

$3 \quad 859 \quad 30.21 \quad 19 \quad 27.99 \quad 155 \quad 26.31$

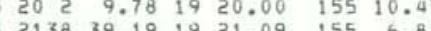
322324.31 19 23.64 15516.89

$34114.24 \quad 1959.34 \quad 155 \quad 43.82$
350 $35050.021920 .05 \quad 1558.13$
6.95 $5250 \quad 29.66 \quad 19 \quad 23.25 \quad 155 \quad 16.94$

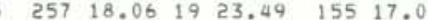

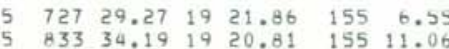

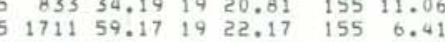
$1753 \quad 31.631920 .33 \quad 155 \quad 13.09$
50.03 $213919.841924 .81 \quad 15525.01$
0

$\begin{array}{lllllll}6 & 047 & 31.78 & 19 & 22.35 & 155 & 6.45 \\ 6 & 258 & 47.30 & 19 & 20.40 & 155 & 7.03\end{array}$

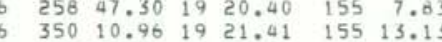
$610 \quad 12.33 \quad 19 \quad 19.26 \quad 15513.46$ $626 \quad 58.13 \quad 19 \quad 21.53 \quad 155 \quad 7.61$

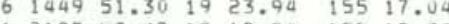
$\begin{array}{lllllll}6 & 2127 & 37.47 & 19 & 19.24 & 155 & 12.38\end{array}$ 6.25150 .6019
23.60 $\begin{array}{ll}14 & 17.78 \quad 1925.05 \quad 155 \\ 14 & 24.40\end{array}$

$\begin{array}{lllllll}7 & 1435 & .04 & 19 & 19.59 & 155 & 16.57\end{array}$

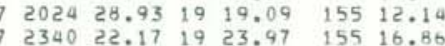

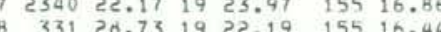
$\begin{array}{lllllll}8 & 331 & 20.73 & 19 & 22.19 & 155 & 16.44 \\ 8 & 550 & 32.12 & 19 & 19.87 & 155 & 12.50\end{array}$

$\begin{array}{lllllll}8 & 552 & 10.89 & 19 & 20.75 & 155 & 13.63\end{array}$

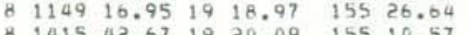
$\begin{array}{lllllll}8 & 1415 & 42.67 & 19 & 20.09 & 155 & 10.57 \\ 8 & 1850 & 26.35 & 19 & 25.54 & 155 & 16.73\end{array}$

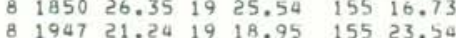

$\begin{array}{llllll}21 & 2 & 42.43 \quad 19 & 23.99 \quad 155 & 16.26\end{array}$

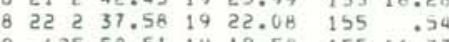

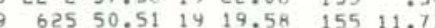

$9.403 .133 .832 \quad 0 \quad 61.1040 .5$. 3004

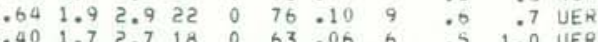

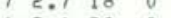

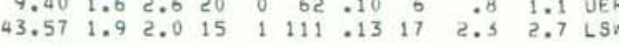
$\begin{array}{lllllllllll}1.45 & 1.4 & 2.6 & 14 & 0 & 61 & .07 & 3 & .4 & .2 & \text { SPC }\end{array}$

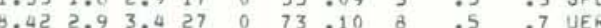

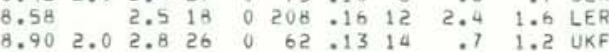
$\begin{array}{llllllllll}11.59 & 2.7 & 22 & 0 & 64 & .13 & 14 & .9 & .4 & \text { UKF }\end{array}$

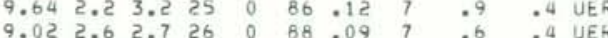

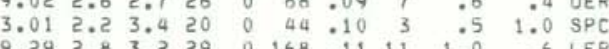

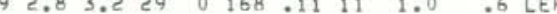
$\begin{array}{lllllllllll}2.89 & 2.7 & 2.8 & 29 & 5 & 143 & .15 & 37 & .7 & 1.0 & \mathrm{KOH}\end{array}$

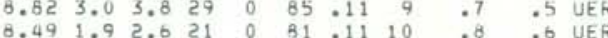

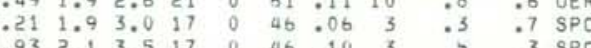
$\begin{array}{llllllll}6.77 & 1.9 & 3.1 & 22 & 0 & 77 & .13 & 7\end{array}$

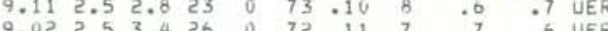
$\begin{array}{lllllllllll}7.67 & 2.7 & 18 & 0 & 65 & .11 & 6 & .8 & 1.7 & \text { UER }\end{array}$

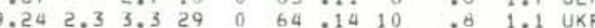

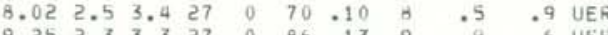

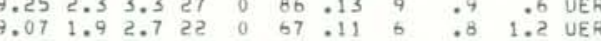

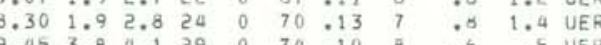
$\begin{array}{lllllllllll}1.77 & 1.9 & 2.6 & 17 & 0 & 61 & .09 & 3 & .4 & .3 & \text { SPC }\end{array}$ $\begin{array}{llllllllllll}3.88 & 1.7 & 2.7 & 24 & 0 & 91 & .13 & 7 & .8 & 1.6 & .3 & \text { UER }\end{array}$

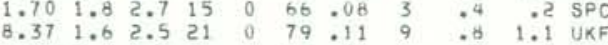

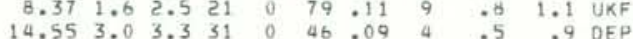

$\begin{array}{lllllllllll}6.49 & 1.5 & 2.4 & 20 & 0 & 99.14 & 5 & .9 & 1.6 & \times 04\end{array}$

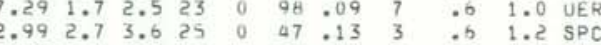
$\begin{array}{lllllllllll}13.90 & 1.8 & 2.2 & 23 & 0 & 52 & .11 & 4 & .0 & 1.1 & \text { DEP }\end{array}$

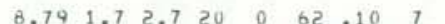

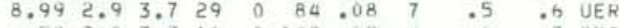

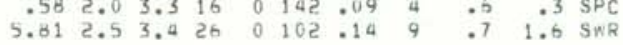
$\begin{array}{lllllllllll}2.00 & 1.8 & 2.6 & 14 & 0 & 100 & .14 & 5 & .9 & .0 & \mathrm{SPC}\end{array}$

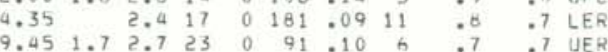

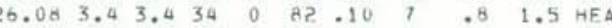




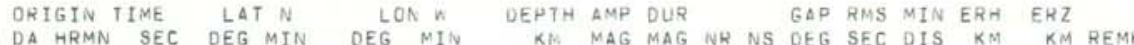
976 DEC $9652 \quad 26.911921 .16 \quad 15513.11$

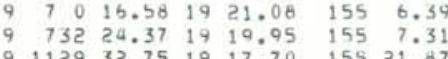
$91345 \quad 55.47 \quad 19$
$23.41 \quad 155 \quad 16.90$

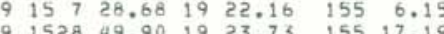
41528 व9.90 1923.75 155 17.19 $9.22930 .121919 .76 \quad 15526.57$

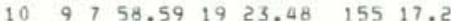

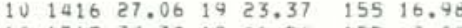
$10 \quad 171533.1919 \quad 41.54 \quad 155 \quad 2.02$ 10172959.801943 .14 155 ट.ट5 10 1750 10.371927 .07 155 29.45

$11 \quad 1255 \quad 47.62 \quad 1922.79 \quad 155 \quad 6.46$ $111751 \quad 47.04 \quad 1920.75 \quad 155 \quad 12.0$ $1253956.541412 .14 \quad 15528.15$ $121210456.801913 .62 \quad 15527.69$

$\begin{array}{llllllll}12 & 14 & 5 & 44.83 & 19 & 24.89 & 155 & 16.13\end{array}$

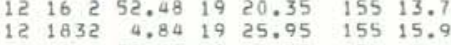
$\begin{array}{lllllll}12 & 1841 & 37.39 & 19 & 19.77 & 155 & 8.10 \\ 12 & 2336 & 38.55 & 19 & 21.49 & 155 & 3.73\end{array}$ $\begin{array}{lllllll}12 & 2357 & 29.50 & 19 & 25.13 & 155 & 24.70\end{array}$

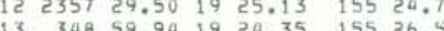
$\begin{array}{llllllll}13 & 5 & 0 & .12 & 19 & 23.42 & 155 & 17.00\end{array}$ $\begin{array}{rrrrrrrr}13 & 6 & 8 & 17.28 & 19 & 19.21 & 155 & 12.05 \\ 13 & 1726 & 42.07 & 19 & 20.20 & 155 & 7.42\end{array}$

$13 \quad 2241 \quad 58.50 \quad 19 \quad 21.24 \quad 155 \quad 13.44$ $\begin{array}{llllllll}14 & 4 & 6 & 22.77 & 19 & 22.38 & 154 & 50.00\end{array}$ $14 \quad 636 \quad 30.37 \quad 1919.83 \quad 155 \quad 12.63$

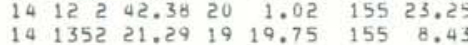
$\begin{array}{lllllll}14 & 1725 & 32.35 & 19 & 24.80 & 155 & 28.70\end{array}$ $\begin{array}{rrrrrrr}15 & 120 & 24.52 & 19 & 27.67 & 155 & 21.64 \\ 15 & 330 & 9.83 & 19 & 26.11 & 154 & 53.03\end{array}$ $15 \quad 440 \quad 42.9419 \quad 19.60$ 155 11.94 $15 \quad 2210 \quad 34.16 \quad 19 \quad 17.22 \quad 155 \quad 21.67$

$\begin{array}{lllllllll}16 & 5 & 4 & 15.78 & 19 & 32.32 & 155 & 26.82\end{array}$

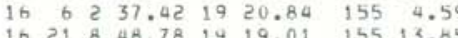
16212135.551925 .32 155 17.05

$\begin{array}{lllllll}17 & 535 & 16.12 & 19 & 25.57 & 155 & 16.50\end{array}$ $\begin{array}{rrrrrrr}17 & 339 & 14.61 & 19 & 25.69 & 155 & 16.70 \\ 17 & 1916 & 54.17 & 19 & 20.40 & 155 & 7.72\end{array}$ $\begin{array}{lllllllllll}8.00 & 2.1 & 3.1 & 29 & 0 & 57 & .12 & 6 & .6 & 1.0 & \text { UER }\end{array}$

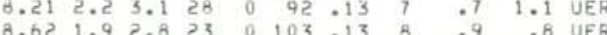

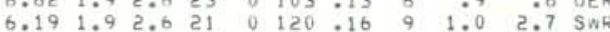

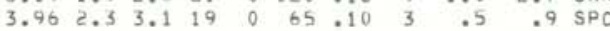
$\begin{array}{lllllllllllll}8.26 & 2.2 & 3.4 & 24 & 0 & 107 & .11 & 7 & .7 & .7 & \text { UER }\end{array}$

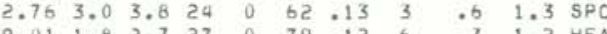

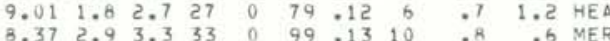

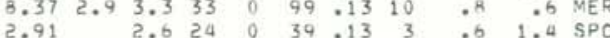

$\begin{array}{lllllllllll}1.82 & 2.1 & 3.0 & 20 & 0 & 46 & .12 & 3 & .6 & .3 & \mathrm{SPC}\end{array}$

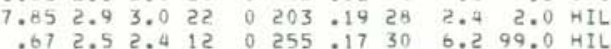
$9.752 .22 .126 \quad 0072.11120 .0 \% 9$ UKF

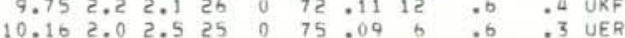

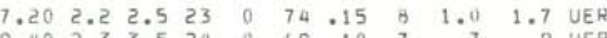

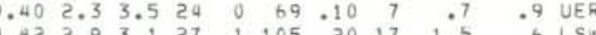

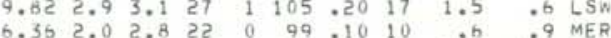

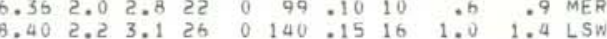

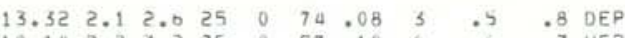

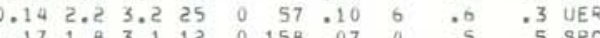

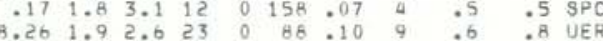

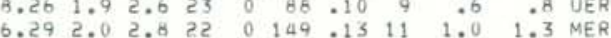

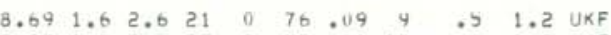

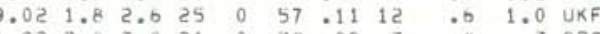

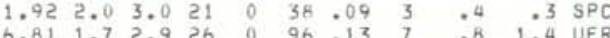

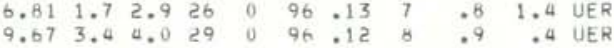
$\begin{array}{lllllllllll}9.28 & 1.9 & 2.5 & 23 & 0 & 56 & 10 & 6 & .7 & .9 & \text { UER }\end{array}$

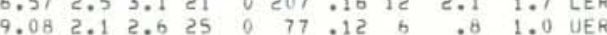

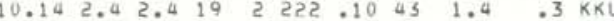
$8.752 .22 .826 \quad 0 \quad 81.1010 \quad .5$.6 UER $\begin{array}{lllllllllllll}9.81 & 2.2 & 2.9 & 32 & 0 & 42 & .13 & 13 & .7 & .4 & \text { UKF }\end{array}$

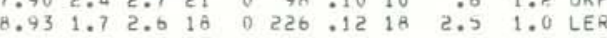
$9.012 .93 .533 \quad 0 \quad 89.12 \quad 6 \quad .7 \quad 0.3$ UER

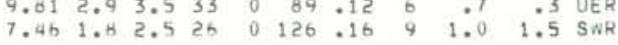
$\begin{array}{rrrrrrrrrrr}25.15 & 2.2 & 2.5 & 31 & 0 & 67 & .10 & 17 & .7 & 1 . R & \text { NER }\end{array}$

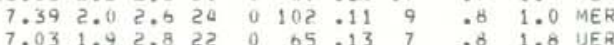

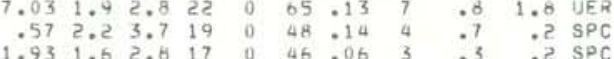
$\begin{array}{lllllllllll}.46 & 1.7 & 3.2 & 15 & 0 & 126 & .16 & 4 & 1.0 & .6 & \mathrm{SPC}\end{array}$

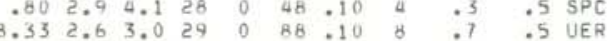

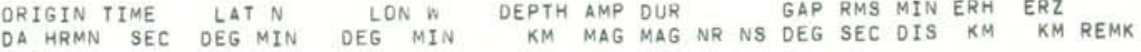
1976 DEC $17 \quad 2031 \quad 43.29 \quad 1920.00 \quad 155 \quad 6.92$

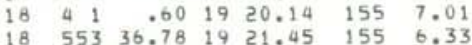
18 612 27.811921 .10 155 7.75 $\begin{array}{lllllll}18 & 814 & 39.99 & 19 & 20.27 & 155 & 7.49\end{array}$ $\begin{array}{lllllll}18 & 814 & 39.99 & 19 & 20.27 & 155 & 7.49 \\ 18 & 857 & 47.82 & 19 & 13.44 & 155 & 30.07\end{array}$ 18 1050 39.491923 .96 155 15.3

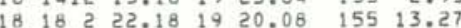
$\begin{array}{lllllll}19 & 110 & 33.88 & 19 & 21.66 & 155 & 7.83\end{array}$ $19 \quad 145 \quad 7.14 \quad 1921.38 \quad 155 \quad 8.29$ 19165036.911920 .391550 .04 $20 \quad 117 \quad 53.651920 .26 \quad 155 \quad 6.60$ $\begin{array}{lllllll}20 & 1246 & 1.42 & 19 & 21.41 & 155 & 7.95\end{array}$ $20 \quad 1253 \quad 7.491921 .39 \quad 155 \quad 7.56$ 20133013.981921 .81 155 15.10 $201932 \quad 28.541923 .15 \quad 15525.4$

$\begin{array}{lllllll}20 & 2317 & 55.16 & 19 & 21.41 & 155 & 2.32\end{array}$ $\begin{array}{lllllll}21 & 224 & 19.42 & 19 & 23.87 & 155 & 16.96\end{array}$ $\begin{array}{lllllll}21 & 245 & 32.26 & 19 & 19.55 & 155 & 15.80\end{array}$ 21 $52934.491920 .91 \quad 15512.98$

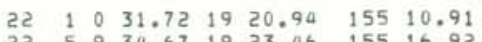
$\begin{array}{llllll}5 & 234.67 & 19 & 23.46 & 155 & 16.92\end{array}$

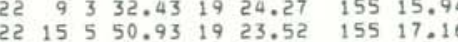
$23 \quad 426 \quad 6.76 \quad 19 \quad 20.15 \quad 15512.20$ $\begin{array}{lllllll}23 & 730 & 24.96 & 19 & 19.27 & 155 & 26.68\end{array}$ $23 \quad 1125 \quad 3.65 \quad 1919.34 \quad 155 \quad 15.67$ $\begin{array}{lllllll}23 & 1349 & 45.80 & 19 & 27.19 & 154 & 53.42\end{array}$

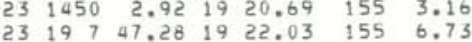

$\begin{array}{lllllll}23 & 2141 & 47.75 & 19 & 27.46 & 154 & 53.39\end{array}$ $\begin{array}{llllll}23 & 221251.4519 & 27.24 & 154 & 53.06\end{array}$ $24 \quad 133 \quad 42.001929 .42 \quad 15454.94$ $\begin{array}{lllllll}24 & 1150 & 31.73 & 19 & 24.38 & 155 & 16.17 \\ 24 & 22 & 20.45 & 19 & 23.36 & 155 & 16.96\end{array}$ $\begin{array}{lllllll}25 & 640 & 3.89 & 19 & 20.29 & 155 & 8.43\end{array}$

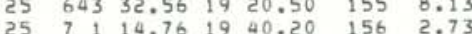
$\begin{array}{lllllll}25 & 813 & 7.45 & 19 & 22.54 & 155 & 4.87\end{array}$

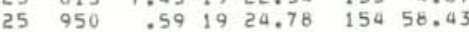

$\begin{array}{llllllll}25 & 22 & 0 & 21.48 & 19 & 23.10 & 155 & 17.31\end{array}$

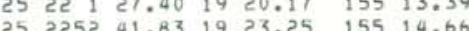

$\begin{array}{lllllllllll}9.49 & 3.2 & 3.7 & 31 & 0 & 109 & .09 & 7 & .7 & \text {.5 UER }\end{array}$ 8.472 .12 .828 0 $85: 12 \quad 7 \quad .7 \quad 99$ UER $\begin{array}{lllllllllll}8.36 & 2.5 & 2.8 & 29 & 0 & 78 & .11 & 9 & .6 & .7 & \text { UER } \\ 6.79 & 2.3 & 3.0 & 25 & 0 & 75 & .12 & 8 & .7 & 1.1 & \text { UER }\end{array}$ $\begin{array}{llllllllllll}8.91 & 2.4 & 2.8 & 28 & 0 & 93 & .13 & 8 & .9 & .6 & .6 & \text { UER }\end{array}$

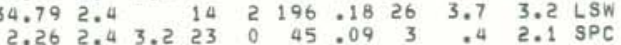

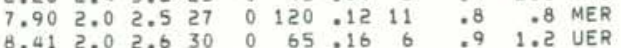
$\begin{array}{lllllllllll}6.53 & 2.8 & 3.3 & 14 & 0 & 71 & .10 & 9 & .8 & 1.5 & \text { UER }\end{array}$

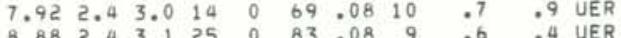

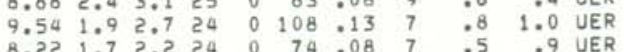

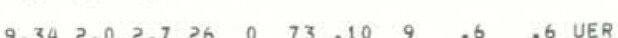

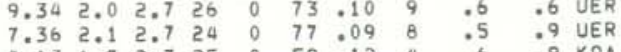
$8.131 .72 .725 \quad 0059.12 \quad 4 \quad .6 \quad .9$ KOA

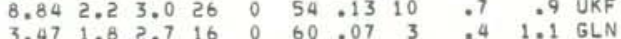

$\begin{array}{lllllllllll}6.65 & 2.1 & 2.7 & 23 & 0 & 152 & .09 & 12 & .8 & .7 & \text { MER }\end{array}$

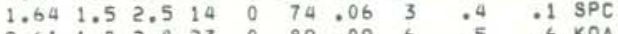

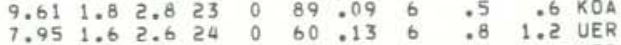

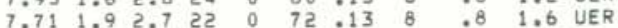

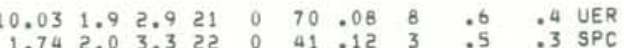

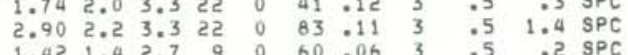

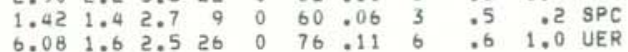
$\begin{array}{llllllllll}8.86 & 2.3 & 3.1 & 29 & 0 & 80 & .17 & 6 & .9 & 1.4 \\ 7 & \text { HEA }\end{array}$ $\begin{array}{llllllllll}7.59 & 2.4 & 18 & 0 & 106 & .07 & 6 & .5 & 1.0 & \mathrm{KOA}\end{array}$ $\begin{array}{lllllllllll}8.83 & 2.4 & 3.1 & 21 & 0 & 231 & .14 & 19 & 2.3 & .7 & \text { LER }\end{array}$

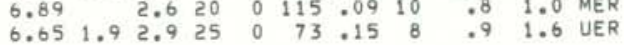
$\begin{array}{llllllllll}8.41 & 1.7 & 2.3 & 13 & 0 & 231 & .16 & 19 & 3.4 & 1.3\end{array}$ LER

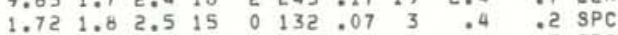
$\begin{array}{llllllllll}1.86 & 1.7 & 2.9 & 18 & 0 & 62.08 & 3 & .4 & .3 & \mathrm{SPC}\end{array}$ $\begin{array}{llllllllllll}8.00 & 1.8 & 2.5 & 25 & 0 & 77 & .11 & 9 & .7 & .6 & \text { UER } \\ 9 & .53 & 3 & 3.4 & 25 & 0 & 80 & .09 & 9 & .7 & .4 & \text { UER }\end{array}$

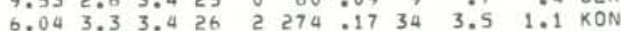

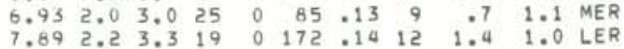
$\begin{array}{lllllllllll}11.83 & 1.7 & 2.3 & 24 & 0 & 50 & .06 & 3 & .5 & .2 & \text { LPC }\end{array}$

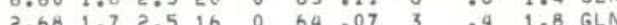

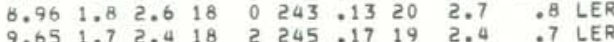




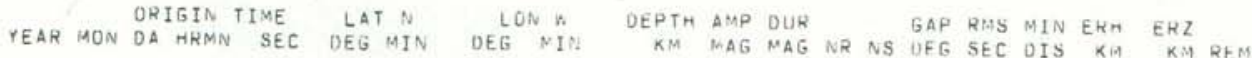

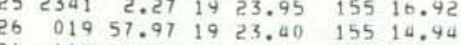
$26 \quad 113 \quad 52.07 \quad 19 \quad 19.76 \quad 155 \quad 11.41$ $26 \quad \begin{array}{lllll}229 & 27.16 & 19 & 19.52 & 155 \\ 26 & 52.67\end{array}$

$26 \quad 632 \quad 44.4019 \quad 23.16 \quad 155 \quad 14.54$ $26 \quad 64931.721923 .23 \quad 15514.55$ 26175331.541925 .9315516 .87 20 $1835 \quad 51.58 \quad 14 \quad 25,92 \quad 155 \quad 28.49$

$\begin{array}{lllllll}26 & 1843 & 17.19 & 19 & 22.82 & 153 & 25.07\end{array}$ $\begin{array}{llllllllll}26 & 2343 & 9.16 & 19 & 47.76 & 155 & 58.54\end{array}$ 27 326 19.731918 .74 155 23.17 $27 \quad 41520.581923 .5015526 .04$

$\begin{array}{lllllll}27 & 526 & 2.45 & 19 & 23.31 & 155 & 14.00\end{array}$

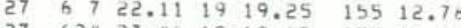
ह7 624 27.46 $1919.45 \quad 15516.55$ $27,121354.0119$ 24.00 15514.84

$\begin{array}{lllllll}27 & 1637 & 24.48 & 19 & 23.22 & 155 & 17.11\end{array}$

or $\quad 27 \quad 1730 \quad 1.841920 .36 \quad 155 \quad 13.44$

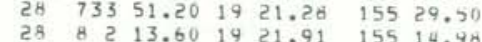

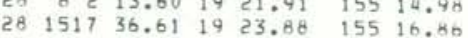
$28 \quad 1624 \quad 41.58 \quad 14 \quad 18.38 \quad 155 \quad 23.50$ 28 $1937 \quad 4.91 \quad 19 \quad 19.2115513 .16$ $29 \quad \begin{array}{llllll}159 & 18.76 & 19 & 22.00 & 155 & 18.17\end{array}$ $29 \quad 34531.311923 .32 \quad 13517.19$ $\begin{array}{llllllll}29 & 4 & 0 & 7.59 & 19 & 23.31 & 155 & 17.07\end{array}$ $2945211.121929 .40 \quad 15449.05$

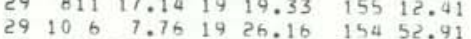
29111025.46 19 23.51 135 34.42 $29124218.23 \quad 19 \quad 19.80 \quad 155 \quad 11.17$

$29 \quad 15 \quad 16.401923 .12 \quad 15514.61$ $291810 \quad 0.63 \quad 19 \quad 19.84 \quad 15511.26$ $291926 \quad 26.92 \quad 1923.48 \quad 155 \quad 14.49$ 24 2033 $20.16 \quad 1920.53 \quad 155 \quad 11,37$

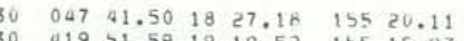
31 व4? 9.411917 .52 155 15.43 $31 \quad 184152.01 \quad 1923.04 \quad 155 \quad 17.45$

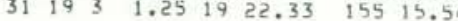

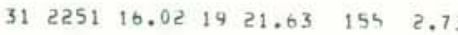

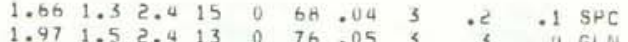

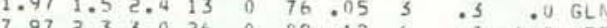
8.351 .92 .619 0 74.0940 .71090 UER

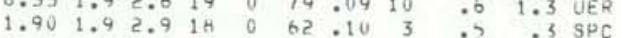
$\begin{array}{lllllllllll}3.52 & 1.7 & 2.5 & 14 & 0 & 59 & 0.116 & 3 & .5 & 1.2 & \text { GLV }\end{array}$

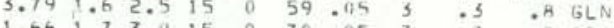

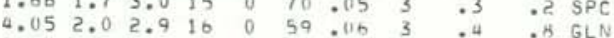

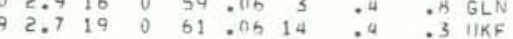
$\begin{array}{lllllllllll}9.56 & 1.7 & 2.8 & 22 & 0 & 56 & 07 & 10 & .4 & .6 & \text { UKF }\end{array}$ 5.661 .53 .519 O 250.19 4e 3.01 .6 KON

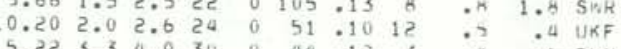
(4) GLN

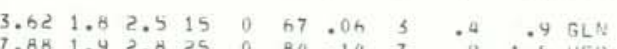

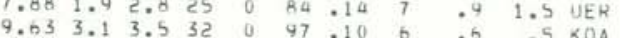

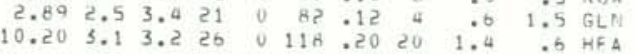
$\begin{array}{lllllllllll}1.59 & 1.3 & 2.2 & 11 & 0 & 59 & .06 & 5 & .4 & .5 & 5 P C\end{array}$

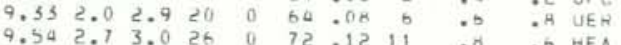

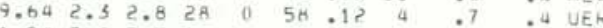

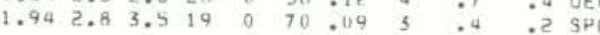
$\begin{array}{llllllllllllll}5.15 & 2.1 & 2.7 & 19 & 0 & 107 & 00 & 4 & .5 & .8 & 5 \mathrm{wm}\end{array}$

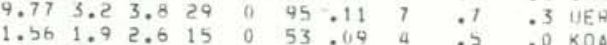

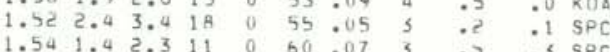

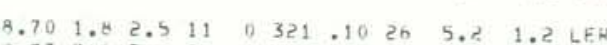

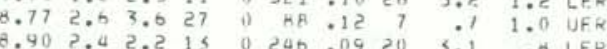

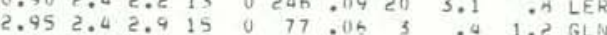

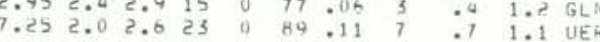
$\begin{array}{lllllllllll}3.54 & 2.1 & 2.9 & 14 & 0 & 61 & .05 & 3 & .5 & .8 & G L\end{array}$

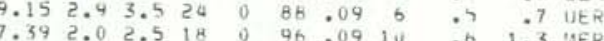

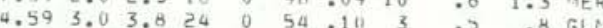

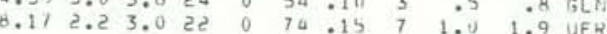

$11.663 .44 .190 \quad 316.074223 .5$.0 DIS

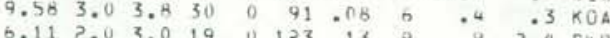

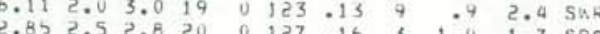

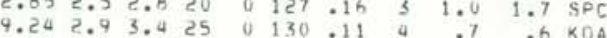

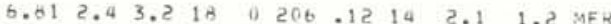


TABLE 6.

OR IGIN TIME YEAR MON DA HRMN SEC DEG MIN 1976

$\begin{array}{rrrrrr}1 & 0 & 2 & 48.09 & 18 & 50.09 \\ 1 & 854 & 19.82 & 18 & 40.44 \\ 1 & 1536 & 47.31 & 19 & 21.71 \\ 7 & 016 & 39.36 & 19 & 18.69 \\ 7 & 144 & 33.33 & 19 & 27.20\end{array}$

$11 \quad 14 \quad 5 \quad 40.17 \quad 19 \quad 30.77$ $\begin{array}{llllll}15 & 1241 & 45.31 & 19 & 24.55\end{array}$

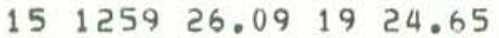
$\begin{array}{llllll}16 & 1929 & 13.84 & 19 & 22.49\end{array}$ $\begin{array}{llllll}16 & 20 & 0 & 3.85 & 19 & 21.29\end{array}$

$\begin{array}{llllll}18 & 449 & 28.37 & 19 & 21.73\end{array}$ $\begin{array}{lllllll}18 & 14 & 5 & 10.92 & 19 & 21.77\end{array}$ $\begin{array}{llllll}18 & 1413 & 37.39 & 19 & 21.65\end{array}$ $\begin{array}{lllll}18 & 2357 \quad 46.47 \quad 1922.56\end{array}$ $21 \quad 114121.241922 .02$

$\begin{array}{lllll}23 & 247 \quad 40.05 \quad 1921.63\end{array}$

$\begin{array}{llllll}23 & 820 & 14.11 & 19 & 21.82\end{array}$

$\begin{array}{lllll}27 & 535 & 51.38 & 19 & 21.20\end{array}$

$\begin{array}{llllll}27 & 2226 & 28.16 & 19 & 20.28\end{array}$

$\begin{array}{llllll}29 & 1019 & 56.47 & 19 & 22.74\end{array}$

$\begin{array}{llllll}31 & 20 & 7 & 26.96 & 19 & 22.10\end{array}$ FEB

$\begin{array}{llllll}3 & 1642 & 13.45 & 19 & 22.68\end{array}$

$\begin{array}{llllll}3 & 2050 & 58.46 & 19 & 21.67\end{array}$

$\begin{array}{llllll}5 & 1149 & 4.48 & 19 & 21.42\end{array}$

$\begin{array}{llllll}5 & 1919 & 16.71 & 19 & 20.45\end{array}$

$\begin{array}{lllll}7 & 2248 & 12.26 & 19 & 19.77\end{array}$

$\begin{array}{lllll}10 & 1820 & 0.55 \quad 19 & 20.33\end{array}$

$\begin{array}{lllll}12 & 618 & 26.21 & 19 & 21.67\end{array}$

$\begin{array}{lllll}13 & 049 & 51.86 & 19 & 31.33\end{array}$

18184052.611925 .82

$\begin{array}{lllll}20 & 1951 & 14.91 & 20 & 24.95\end{array}$

$\begin{array}{lllll}24 & 550 & 19.19 & 19 & 22.20\end{array}$

$241442 \quad 32.631923 .27$

$\begin{array}{llllll}24 & 1918 & 30.56 & 19 & 21.12\end{array}$

$\begin{array}{llllll}25 & 1348 & 20.37 & 19 & 22.17\end{array}$

$\begin{array}{lllll}28 & 2355 & 25.89 & 19 & 22.42\end{array}$ $\begin{array}{llllll}29 & 1941 & 58.83 & 19 & 20.28\end{array}$

$\begin{array}{llllll}\text { MAR } & 6 & 1223 & 4.59 & 19 & 21.60\end{array}$

$\begin{array}{lllll}7 & 1625 & 34.64 & 19 & 21.78\end{array}$

$95517.66 \quad 1918.54$

$\begin{array}{lllll}10 & 1832 & 40.95 & 19 & 20.30\end{array}$

114242.231921 .60

$13616 \quad 16.38 \quad 19 \quad 8.69$

$191324 \quad 30.321921 .32$

$\begin{array}{llllll}20 & 1313 & 19.86 & 19 & 19.83\end{array}$

$\begin{array}{lllll}21 & 931 & 33.54 & 19 & 22.23\end{array}$

$\begin{array}{llllll}21 & 2031 & 24.60 & 19 & 20.22\end{array}$

$\begin{array}{llllll}22 & 2048 & 26.94 & 19 & 21.85\end{array}$
LON W DEG MIN

1554.68

1552.80

$155 \quad 15.08$

15520.66

15453.21

$155 \quad 16.17$

$155 \quad 17.59$

$155 \quad 17.60$

1556.53

$154 \quad 59.61$

$155 \quad 15.30$

1557.01

1557.62

$155 \quad 5.92$

1557.21

1554.98

1557.92

1556.17

1556.64

15459.64

1554.83

$155 \quad 5.95$

$155 \quad 15.18$

1558.12

1557.11

$155 \quad 7.46$

$155 \quad 6.87$

1553.51

15557.70

15516.46

$156 \quad 1.29$

$155 \quad 6.65$

1554.81

1557.96

1556.35

$155 \quad 6.36$

1550.61

$155 \quad 1.95$

1557.17

15520.65

$155 \quad 7.13$

$155 \quad 15.45$

15530.14

1553.43

1556.53

$155 \quad 15.26$

1558.61

1555.03
DEPTH AMP DUR

GAP RMS MIN ERH ERZ

KM MAG MAG NR NS DEG SEC UIS KM

KM REMK
$3.929 .5 \mathrm{PPL}$ 6.014 .1 PPL

$.4 .5 \mathrm{KOA}$

$.7 \quad .4$ SWR

.9 .5 LER

24.494 .24 .232

$14.21 \quad 4.4 \quad 4.5 \quad 35$

$16.07 \quad 4.5 \quad 4.7 \quad 34$

$8.43 \quad 3.7 \quad 4.0 \quad 29$

$\begin{array}{llll}2.18 & 3.2 & 3.8 & 25\end{array}$

$9.70 \quad 3.6 \quad 3.8 \quad 31$

$9.35 \quad 3.6 \quad 4.0 \quad 30$

$\begin{array}{lllll}7.03 & 3.6 & 3.7 & 31\end{array}$

$7.94 \quad 3.6 \quad 3.5 \quad 32$

$8.48 \quad 4.14 .224$

$\begin{array}{lllll}8.96 & 3.7 \quad 4.0 & 31\end{array}$

$8.913 .2 \quad 3.526$

$8.693 .6 \quad 3.8 \quad 31$

$9.25 \quad 4.0 \quad 4.131$

$8.664 .7 \quad 4.9 \quad 33$

$\begin{array}{llll}7.52 & 3.3 & 3.9 & 28\end{array}$

$8.74 \quad 3.4 \quad 3.7 \quad 30$

$9.78 \quad 3.5 \quad 3.8 \quad 29$

8.143 .13 .628

$9.27 \quad 3.23 .628$

$\begin{array}{lllll}7.82 & 3.2 & 3.7 & 27\end{array}$

$9.50 \quad 2.8 \quad 3.5 \quad 25$

$\begin{array}{llll}7.25 & 3.2 \quad 3.8 \quad 28\end{array}$

$\begin{array}{lllll}9.68 & 3.6 & 3.1 & 27\end{array}$ $\begin{array}{llll}.69 & 2.8 & 4.1 & 21\end{array}$

$31.75 \quad 5.14 .6 \quad 5$ 8.594 .24 .229

$\begin{array}{llll}7.82 & 3.2 & 3.6 & 23\end{array}$

$9.38 \quad 3.13 .5 \quad 31$

$8.98 \quad 3.9 \quad 4.2 \quad 31$

61.1113

.71 .8 GLN

.5 .8 DEP

.61 .0 DEP

.7 .6 UER

1.2 1.5 LEK

$0 \quad 68.09 \quad 8$

0206.1212

$0 \quad 60.09 \quad 4$

$0 \quad 76.10$

$0 \quad 73.09$

$\begin{array}{llll}0 & 71 & .107\end{array}$

0178.1210

$0 \quad 82.139$

$0 \quad 79.10$

$0 \quad 91.11$

$0 \quad 107.10$

$0 \quad 184.1410$

82.139

72.117

61.08

71.12

96.08

\section{.5}

.6

.5

.3 KOA

.5

1.3

.7 UER

.7 MER

.8 .6 MER

$.6 \quad .7$ UER

$.7 \quad .5$ UER

0

1.2

.5 UER

.7 .9 MER

.6 . . . MER

$.4 .3 \mathrm{KOA}$

.7 .9 UER

.6 . 4 UER

$8.12 \quad 3.3 \quad 3.6 \quad 25$

$9.13 \quad 3.13 .5 \quad 27$

$6.04 \quad 3.5 \quad 4.0 \quad 29$

$8.243 .7 \quad 4.030$

$\begin{array}{llll}0 & 103.098\end{array}$

.5

. 8 UER

.6 .6 UER

.8 .8 MER

2.1. $4 \mathrm{KON}$

.61 .2 SPC

$9.37 \quad 2.8 \quad 3.5 \quad 30$

$9.052 .8 \quad 3.6 \quad 26$

$8.98 \quad 3.1 \quad 3.6 \quad 25$

$11.26 \quad 3.5 \quad 3.5 \quad 28$

8.113 .94 .129

$9.213 .1 \quad 3.5 \quad 27$

0162.0083

$\begin{array}{rrrr}0 & 71 & .11 & 8 \\ 0 & 129 & 11 & 9\end{array}$

$0 \quad 76.119$

073.09

34.0

$.6 \quad .7$ UER

.1 1.0 MER

.7 .4 UER

.5 .4 UER

.6 .8 UER

.8 . 5 UER

.7 1.1 MEK

.6 . 6 UER

.5 .4 SWR

$9.78 \quad 3.3 \quad 3.6 \quad 20$

$8.42 \quad 3.2 \quad 3.6 \quad 27$

$8.75 \quad 3.3 \quad 3.5 \quad 29$

55
$.9 \quad .7$ UER

$.7 .8 \mathrm{KOA}$

$1.0 .4 \mathrm{LSW}$

.8 1.0 MER

.7 .5 UER

$.81 .6 \mathrm{KOA}$

.6 .7 UER

.7 .6 MER 
ORIGIN TIME LAT N LON W YEAR MON DA HRMN SEC DEG MIN DEG MIN

1976 MAR $23 \quad 15 \quad 6 \quad 3.24 \quad 19 \quad 18.82$ $\begin{array}{lllll}26 & 844 & 47.75 & 19 & 20.67\end{array}$ $295952.00 \quad 1921.83$ $\begin{array}{llll}30 & 1452 & 10.26 \quad 19 & 20.01\end{array}$

$A P R \quad 2 \quad 814 \quad 6.501920 .62$

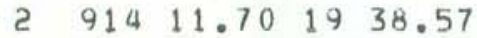

$\begin{array}{llllll}2 & 955 \quad 3.26 & 19 & 19.86\end{array}$

$\begin{array}{lllll}3 & 143 \quad 42.22 & 19 & 23.72\end{array}$

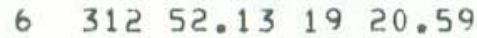

$11 \quad 1535 \quad 49.18 \quad 19 \quad 20.95$

$\begin{array}{lllll}12 & 726 & 23.45 \quad 19 & 18.94\end{array}$ 1422136.021927 .63 $\begin{array}{llllll}20 & 711 & 3.11 & 19 & 21.70\end{array}$ $21 \quad 1813 \quad 34.49 \quad 18 \quad 47.70$ 22 $554 \quad 4.92 \quad 1931.50$

$\begin{array}{lllll}22 & 833 \quad .97 \quad 19 \quad 18.57\end{array}$ $\begin{array}{lllll}23 & 1229 & 53.77 & 19 & 21.88\end{array}$ $\begin{array}{llllll}26 & 2144 & 41.56 & 19 & 23.26\end{array}$ MAY 118524.851918 .43

$9 \quad 614 \quad 43.37 \quad 1921.55$

$\begin{array}{lllll}10 & 827 & 4.45 & 19 & 23.27\end{array}$

$1255511.56 \quad 1920.33$

$\begin{array}{llllll}17 & 145 & 6.09 & 19 & 19.78\end{array}$

$172054 \quad 49.19 \quad 19 \quad 19.66$

$\begin{array}{lllll}18 & 616 & 41.31 & 19 & 19.76\end{array}$

$\begin{array}{lllll}19 & 1712 & 5.53 \quad 19 & 19.80\end{array}$

$\begin{array}{llllll}21 & 1953 & 9.83 & 19 & 21.08\end{array}$

$22 \quad 1852 \quad 8.44 \quad 1921.68$

$\begin{array}{llllll}23 & 1731 & 20.18 & 19 & 20.44\end{array}$

$232324 \quad 7.6320 \quad 54.93$

$\begin{array}{llllll}28 & 12 & 8 & 45.51 & 19 & 23.03\end{array}$

$\begin{array}{lllll}31 & 627 & 21.09 & 20 & 14.77\end{array}$

$31832 \quad 9.9920 \quad 15.86$

JUN $42250 \quad 51.27 \quad 1921.54$

$\begin{array}{llllll}7 & 1039 & 37.57 & 19 & 19.15\end{array}$

$\begin{array}{lllll}10 & 015 & 3.85 & 19 & 20.07\end{array}$

$\begin{array}{llllll}10 & 22 & 5 & 5.23 & 19 & 19.78\end{array}$

$\begin{array}{llllll}14 & 1417 & 15.19 & 19 & 24.25\end{array}$

$\begin{array}{lllll}16 & 917 & 15.92 & 19 & 21.81\end{array}$

$\begin{array}{llllll}18 & 12 & 1 & 11.60 & 19 & 22.53\end{array}$

$\begin{array}{llllll}20 & 3 & 6 & 23.81 & 19 & 11.44\end{array}$

$\begin{array}{llllll}21 & 1539 & 5.68 & 19 & 22.49\end{array}$

$\begin{array}{llllll}21 & 1816 & 27.24 & 19 & 22.15\end{array}$

$\begin{array}{lllllll}21 & 19 & 3 & 34.10 & 19 & 22.27\end{array}$

$\begin{array}{llllll}21 & 1936 & 29.86 & 19 & 22.10\end{array}$

$\begin{array}{lllllll}21 & 20 & 0 & 34.66 & 19 & 22.34\end{array}$ $\begin{array}{llllll}21 & 201512.03 \quad 19 & 22.81\end{array}$ $\begin{array}{llllll}21 & 2241 & 19.86 & 19 & 22.36\end{array}$
15518.38

$155 \quad 10.80$

15515.19

1557.32

1556.50

$155 \quad 59.93$

15512.98

15517.02

$155 \quad 12.54$

1553.56

15521.56

$154 \quad 53.07$

$155 \quad 15.25$

$155 \quad .30$

15518.64

15520.64

$155 \quad 5.15$

$155 \quad 16.96$

15523.46

1557.95

$155 \quad 4.48$

15511.66

15516.26

1558.60

1557.48

$155 \quad 7.74$

$155 \quad 6.06$

$155 \quad 15.11$

$155 \quad 6.81$

$\begin{array}{lll}156 & 23.40\end{array}$

1554.84

$155 \quad 53.55$

$155 \quad 46.17$

1557.10

$155 \quad 15.64$

1557.32

$155 \quad 6.51$

15515.84

$155 \quad 4.84$

$155 \quad 6.06$

$155 \quad 32.81$

$\begin{array}{lll}155 & 13.27\end{array}$

$155 \quad 12.92$

$155 \quad 13.13$

15512.95

$155 \quad 13.23$ $155 \quad 5.04$

15512.75

DEPTH AMP DUR

GAP RMS MIN ERH

ERZ

KM MAG MAG NR NS DEG SEC DIS KM KM REMK

$\begin{array}{llllllll}9.48 & 3.7 & 3.9 & 30 & 0 & 84 & .13 & 8\end{array}$

$9.432 .9 \quad 3.5 \quad 18$

$9.73 \quad 3.7 \quad 4.0 \quad 27$

$9.50 \quad 3.4 \quad 3.7 \quad 29$

$9.37 \quad 4.6 \quad 4.6 \quad 33$

$6.64 \quad 3.4 \quad 3.6 \quad 24$

$8.413 .6 \quad 3.928$

$3.913 .0 \quad 3.6 \quad 32$

$9.213 .4 \quad 3.7 \quad 29$

$\begin{array}{llll}5.33 & 3.4 & 3.7 & 26\end{array}$

$9.41 \quad 3.0 \quad 3.5 \quad 27$

$8.16 \quad 3.3 \quad 3.529$

$9.50 \quad 3.5 \quad 3.7 \quad 31$

49.814 .64 .222

$11.84 \quad 3.4 \quad 3.6 \quad 32$

$9.76 \quad 3.4 \quad 3.7 \quad 26$

8.954 .24 .328

5.403 .13 .826

6.633 .24 .031

$9.72 \quad 3.43 .6 \quad 30$

$\begin{array}{rrr}0 & 74 & .08 \\ 0 & 59 & .12 \\ 0 & 101 & .10\end{array}$

$0 \quad 101.10$

2 221.2129

$0 \quad 72.09$

$0 \quad 37.16 \quad 3$

0

67.137

$99.14 \quad 10$

0103.128

$0 \quad 244.15 \quad 19$

$\begin{array}{llll}0 & 60 & .11 \quad 4\end{array}$

0278.0963

$\begin{array}{lll}0 & 61 & 14 \quad 15\end{array}$

$0 \quad 109.11$

$\begin{array}{lll}0 & 771\end{array}$

044.10

$0 \quad 107.17$

$\begin{array}{lllll}7.73 & 3.5 & 3.6 \quad 32\end{array}$

$9.26 \quad 3.7 \quad 4.231$

$9.60 \quad 3.6 \quad 3.8 \quad 32$

$9.513 .0 \quad 3.6 \quad 28$

8.633 .54 .032

$094.12 \quad 10$

$\begin{array}{llll}0 & 77.107\end{array}$

091.095

$0 \quad 78.0910$

$\begin{array}{rrr}0 & 102.128\end{array}$

$\begin{array}{lllll}9.29 & 3.8 & 4.131\end{array}$

$9.27 \quad 3.2 \quad 3.5 \quad 31$

$\begin{array}{lllll}10.51 & 3.7 & 4.0 & 31\end{array}$$$
\text { (0) }
$$

9.384 .004 .031

0 $96.12 \quad 8$

$\begin{array}{llll}0 & 94 & .117\end{array}$

$0 \quad 60.09$

$\begin{array}{llll}0 & 101 & .117 \\ 2 & 206 & .14169\end{array}$

$\begin{array}{lllll}8.11 & 3.3 & 3.7 & 26\end{array}$

$\begin{array}{llll}31.66 & 3.6 & 3.39\end{array}$

$2.68 \quad 4.13 .9 \quad 6$

$9.37 \quad 4.14 .432$

$31.40 \quad 3.5 \quad 3.3 \quad 35$$$
\begin{aligned}
& 0 \\
& 0 \\
& 2
\end{aligned}
$$$$
98.099
$$

$8.55 \quad 3.3 \quad 3.7 \quad 32$

$9.55 \quad 3.2 \quad 3.7 \quad 33$

$4.373 .2 \quad 3.924$

$7.79 \quad 3.4 \quad 3.6 \quad 31$

$7.50 \quad 3.43 .6 \quad 28$$$
\begin{aligned}
& 0 \\
& 0 \\
& 0 \\
& 0 \\
& 0
\end{aligned}
$$

$10.643 .13 .5 \quad 23$ $6.802 .93 .6 \quad 26$

$\begin{array}{llll}5.39 & 3.1 & 3.5 & 28\end{array}$

$6.15 \quad 3.4 \quad 3.7 \quad 27$

6.063 .13 .620

$$
\begin{aligned}
& 0 \\
& 0 \\
& 0 \\
& 0 \\
& 0
\end{aligned}
$$

$5.30 \quad 3.3 \quad 3.926$

$9.38 \quad 3.2 \quad 3.5 \quad 30$ 56

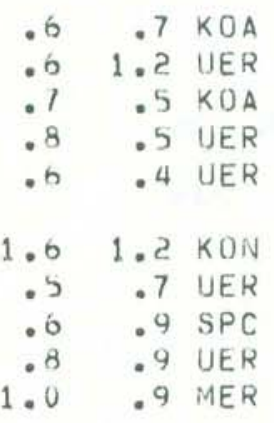

$.7 .8 \quad$ SWR

2.3 .9 LER

$.5 .5 \mathrm{KOA}$

4.89 .0 PPL

.8 .3 NEK

.7 .4 SWR

.7 .6 MER

.4 .6 SPC

.81 .6 SWR

.5 .3 UER

$.5 .8 M E R$

.6 .7 UER

$.5 .4 \mathrm{KOA}$

.6 . 2 UER

.8 . .6 UER

.8 .6 UER

.8 . .5 UER

.5 .2 KOA

.8 .5 UER

3.21 .6 DIS

.6 MER

$9.7 \mathrm{KOH}$

5.6 DIS

.5 UER

1.3 DEP

.7 .6 UER

.6 . . U UER

.81 .0 SPC

.5 . .5 MER

.6 .9 UER

$\begin{array}{llll}4.87 & 2.8 & 3.6 & 28\end{array}$ 6
$1.3 .6 \mathrm{LSW}$

$.5 \quad 1.0$ UER

.5 .5 IJER

.41 .0 UER

.8 2.6 UER

$\begin{array}{lll}.6 & .7 & \text { UEER } \\ .7 & .6 & M E R \\ .1 & .9 & \text { UER }\end{array}$ 
ORIGIN TIME LAT N LON W DEPTH AMP DUR GAP RMS MIN ERH ERZ YEAR MON DA HRMN SEC DEG MIN DEG MIN 1976 JUN 22 $3439.56 \quad 19 \quad 22.64$ $\begin{array}{lllll}22 & 341 & 9.92 & 19 & 23.13\end{array}$ $\begin{array}{lllll}23 & 052 & 25.03 & 19 & 21.58\end{array}$ $\begin{array}{lllll}24 & 1947 & 53.85 \quad 19 & 20.31\end{array}$ $\begin{array}{lllll}25 & 129 & 52.49 & 19 & 23.27\end{array}$

JUL $\quad 5 \quad 140 \quad 43.57 \quad 19 \quad 24.07$

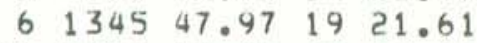
$\begin{array}{llllll}11 & 2326 & 10.83 & 19 & 21.11\end{array}$ $\begin{array}{lllllll}14 & 2 & 5 & 2.37 & 19 & 23.21\end{array}$ $14 \quad 1414 \quad 38.07 \quad 1922.65$

$\begin{array}{lllll}15 & 854 & 39.83 & 19 & 21.66\end{array}$ $\begin{array}{llllll}16 & 111 & 28.75 & 19 & 10.70\end{array}$ $\begin{array}{llllll}18 & 2331 & 25.59 & 19 & 19.83\end{array}$ $22 \quad 240 \quad 53.05 \quad 19 \quad 22.63$ $\begin{array}{lllll}22 & 2149 & 45.42 & 19 & 23.77\end{array}$

$23 \quad 1938 \quad 17.12 \quad 1920.05$ $24 \quad 122 \quad 14.65 \quad 19 \quad 13.35$ $\begin{array}{llllll}24 & 2346 & 30.98 & 19 & 23.34\end{array}$ $\begin{array}{llllll}27 & 714 & 26.85 & 19 & 22.27\end{array}$ $\begin{array}{lllll}27 & 2037 & 12.69 & 19 & 23.49\end{array}$

$\begin{array}{llllll}28 & 16 & 3 & 19.82 & 19 & 23.73\end{array}$ $\begin{array}{llllll}30 & 59 & 43.69 & 19 & 20.40\end{array}$ $\begin{array}{lllll}30 & 2319 & 14.05 & 19 & 20.27\end{array}$

$\begin{array}{llllll}31 & 1651 & 36.05 & 19 & 24.28\end{array}$

AUG $11639 \quad 18.04 \quad 19 \quad 23.58$

$\begin{array}{lllll}2 & 637 & 1.42 & 19 & 21.52\end{array}$

$\begin{array}{lllll}3 & 523 & 31.91 & 19 & 26.50\end{array}$

$\begin{array}{lllll}6 & 338 & 55.38 & 19 & 22.71\end{array}$

$\begin{array}{lllll}7 & 131 & 45.35 & 19 & 21.29\end{array}$

$\begin{array}{lllll}7 & 1142 \quad 19.32 \quad 19 & 19.17\end{array}$

$\begin{array}{llllll}9 & 13 & 9 & 33.49 & 19 & 23.37\end{array}$

$\begin{array}{llllll}10 & 19 & 3 & 47.59 & 19 & 19.17\end{array}$

$\begin{array}{llllll}14 & 17 & 1 & .37 & 19 & 21.44\end{array}$

$\begin{array}{llll}16 & 231 & 8.43 \quad 1921.89\end{array}$

$26 \quad 31943.60 \quad 1921.78$

$\begin{array}{lllll}28 & 1859 & 55.45 \quad 19 & 21.29\end{array}$

$\begin{array}{lllll}29 & 2036 & 25.54 & 19 & 27.89\end{array}$

$\begin{array}{llllll}31 & 1041 & 8.47 & 19 & 23.42\end{array}$

$\begin{array}{llllll}31 & 14 & 0 & 38.53 & 19 & 19.89\end{array}$

$\begin{array}{lllll}2 & 1020 & 26.76 & 19 & 21.18\end{array}$

$\begin{array}{lllll}3 & 116 & 55.89 & 19 & 19.77\end{array}$

$\begin{array}{llllll}3 & 1523 & 20.02 \quad 19 & 22.93\end{array}$

$\begin{array}{lllllll}3 & 22 & 3 & 41.40 & 19 & 28.89\end{array}$

$\begin{array}{lllll}4 & 1310 & 54.24 & 19 & 19.79\end{array}$

6534
$65.20 \quad 19 \quad 26.46$

$\begin{array}{lllll}6 & 1919 \quad .38 & 19 & 21.23\end{array}$

$\begin{array}{lllllll}7 & 221 & 16.28 & 19 & 22.20\end{array}$

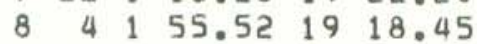

15514.46

15514.60

$155 \quad 15.54$

15511.90

$155 \quad 16.91$

1553.59

15515.27

15513.35

15514.55

15512.76

$155 \quad 7.67$

$155 \quad 32.65$

1557.64

1554.62

15514.85

$155 \quad 8.39$

$155 \quad 35.54$

$155 \quad 14.74$

$155 \quad 4.89$

15514.57

$155 \quad 23.92$

$155 \quad 6.66$

15512.19

15515.86

15514.80

$155 \quad 15.11$

$154 \quad 53.67$

$155 \quad 5.58$

1552.69

15511.98

15514.62

15513.31

1558.07

$155 \quad 3.90$

15515.06

$155 \quad 13.39$

$154 \quad 52.91$

15529.67

1557.01

1553.20

15510.60

15524.70

15450.99

15516.32

15454.22

15516.05

$155 \quad 4.73$

15520.83
KM MAG MAG

DEG SEC DIS KM

KM REMK

$2.53 \quad 3.2 \quad 4.0 \quad 32$

$\begin{array}{lllll}5.37 & 3.0 & 3.5 & 30\end{array}$

$\begin{array}{llll}2.17 & 2.9 & 3.7 & 29\end{array}$

$9.23 \quad 3.6 \quad 3.8 \quad 29$

$\begin{array}{llll}3.77 & 2.5 & 3.5 & 25\end{array}$

$8.08 \quad 3.1 \quad 3.6 \quad 21$

$9.413 .2 \quad 3.528$

$9.48 \quad 3.2 .3 .5 \quad 31$

$\begin{array}{llll}4.89 & 3.3 & 3.7 & 25\end{array}$

$\begin{array}{llll}4.88 & 3.1 & 3.8 & 25\end{array}$

$\begin{array}{lllll}7.81 & 3.3 & 3.5 & 28\end{array}$

$9.32 \quad 3.4 \quad 4.0 \quad 27$

$9.20 \quad 3.7 \quad 4.1 \quad 34$

4.823 .23 .631

$9.55 \quad 3.7 \quad 4.0 \quad 32$

$10.32 \quad 3.4 \quad 3.5 \quad 29$

$\begin{array}{llll}5.03 & 3.3 & 3.9 & 28\end{array}$

$9.39 \quad 4.0 \quad 4.132$

$4.70 \quad 3.6 \quad 4.0 \quad 30$

$9.67 \quad 3.5 \quad 3.3 \quad 31$

$8.04 \quad 3.8 \quad 4.133$

$8.623 .0 \quad 3.531$

$\begin{array}{llll}4.61 & 3.3 & 3.8 & 29\end{array}$

$6.213 .6 \quad 3.8 \quad 25$

$9.873 .2 \quad 3.5 \quad 29$

$\begin{array}{lllll}8.88 & 3.0 & 3.5 & 27\end{array}$

$8.12 \quad 3.2 \quad 3.6 \quad 30$

$\begin{array}{lllll}6.59 & 3.3 & 3.8 & 27\end{array}$

9.063 .13 .823

$\begin{array}{ll}50 & .15 \\ 46 & .11 \\ 62 & .16 \\ 76 & .10 \\ 45 & .14\end{array}$

.6

.4

.6

.5

$0 \quad 85.12$

$\begin{array}{lllll}4.99 & 3.4 & 3.7 & 30\end{array}$

$10.12 \quad 3.5 \quad 3.8 \quad 32$

$8.443 .2 \quad 3.6 \quad 29$

$7.442 .43 .5 \quad 18$

$10.153 .6 \quad 4.031$

$\begin{array}{llll}0 & 98 & 10 & 7\end{array}$

9.663 .03 .630

$7.682 .93 .5 \quad 18$

$10.18 \quad 4.0 \quad 4.135$

9.053 .43 .830

074.107

$\begin{array}{llll}0 & 71 & .10 \quad 9\end{array}$

$7.62 \quad 3.5 \quad 4.0 \quad 31$

$8.90 \quad 3.1 \quad 3.5 \quad 33$

$9.57 \quad 2.9 \quad 3.5 \quad 19$

$\begin{array}{lllll}7.62 & 3.5 & 3.8 & 27\end{array}$

$9.62 \quad 3.9 \quad 4.0 \quad 33$

$\begin{array}{llll}7.97 & 3.5 & 4.0 & 30\end{array}$

040.1313

$\begin{array}{llll}0 & 110.127\end{array}$

4.523 .64 .226

$8.28 \quad 3.5 \quad 4.0 \quad 32$
.7

1. 0

.5

.5

6

.6

.7

.7

072.148

$\begin{array}{llll}0 & 136 & .18 & 23\end{array}$

$98.10 \quad 8$

90.139

$0 \quad 56.14$

$0 \quad 80 \quad .10 \quad 10$

$\begin{array}{llll}0 & 115.19 & 17\end{array}$

045.123

83.12

46.12

0.66 .12

0104.09

075.13

0109.12

$0 \quad 62.105$

$0 \quad 227.0919$

) $76.11 \quad 8$

0140.1112

$045.13 \quad 3$

0114.0810

$0 \quad 59.10$

$0 \quad 55.09 \quad 6$
0

$\begin{array}{llll}0 & 258 \quad .14 \quad 19\end{array}$

0118.1111

\begin{tabular}{|c|c|c|c|c|c|c|}
\hline & 45 & .14 & 3 & .7 & 1.0 & SPC \\
\hline 0 & 119 & .12 & 11 & 1.0 & .8 & MER \\
\hline 0 & 62 & .09 & 4 & .5 & .6 & $K O A$ \\
\hline 0 & 55 & .09 & 6 & .5 & .4 & UER \\
\hline & 55 & .12 & 3 & .6 & .7 & GLN \\
\hline & 97 & .14 & 8 & .7 & 1.0 & UER \\
\hline & 72 & .14 & 8 & .7 & .8 & UER \\
\hline & 136 & .18 & 23 & 1.3 & 1.4 & LSW \\
\hline & 98 & .10 & 8 & .8 & .5 & UER \\
\hline J & 90 & .13 & 9 & .8 & .5 & MER \\
\hline & 56 & .14 & 3 & .5 & .7 & GLN \\
\hline ) & 80 & .10 & 10 & .6 & .3 & UER \\
\hline 0 & 115 & .19 & 17 & 1.2 & .5 & HEA \\
\hline 0 & 45 & .12 & 3 & 5. & .6 & GLN \\
\hline 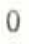 & 83 & .12 & 9 & .9 & .5 & MER \\
\hline & 46 & .12 & 3 & .5 & .6 & GLN \\
\hline 0 & 66 & .12 & 7 & .6 & .5 & UKF \\
\hline 0 & 104 & .09 & 7 & .5 & .4 & UER \\
\hline$)$ & 75 & .13 & 6 & .6 & .6 & UER \\
\hline 0 & 109 & .12 & 3 & .5 & .6 & SPC \\
\hline & 85 & .12 & 6 & .6 & 1.2 & GLN \\
\hline & 62 & .10 & 5 & .6 & .4 & KOA \\
\hline & 227 & .09 & 19 & 1.4 & .5 & LER \\
\hline & 76 & .11 & 8 & .6 & .6 & MER \\
\hline 0 & 140 & .11 & 12 & .8 & .8 & MER \\
\hline & 98 & .10 & 7 & .7 & .9 & UER \\
\hline ) & 45 & .13 & 3 & .5 & .6 & GLN \\
\hline 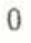 & 74 & .10 & 7 & .6 & .2 & UER \\
\hline$)$ & 71 & .10 & 9 & .5 & .6 & UER \\
\hline 0 & 114 & .08 & 10 & .7 & .6 & $M E R$ \\
\hline 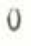 & 59 & .10 & 4 & .6 & .3 & KOA \\
\hline 0 & 55 & .09 & 6 & .5 & .3 & UER \\
\hline 0 & 258 & .14 & 19 & 3.2 & 1.0 & LER \\
\hline 0 & 40 & .13 & 13 & .6 & .3 & UKF \\
\hline 0 & 110 & .12 & 7 & .9 & .6 & UER \\
\hline 0 & 118 & .11 & 11 & .8 & .5 & MER \\
\hline
\end{tabular}

.8 .8 UKF

3.2 .9 LER

.5 .4 KOA

1.7 .7 LER

$.71 .0 \mathrm{KOA}$

.7 .6 MER .5 .3 SWR $\begin{array}{lllll}8.83 & 3.2 & 3.8 & 27\end{array}$ 
ORIGIN TIME LAT N YEAR MON DA HRMN SEC DEG MIN

1976

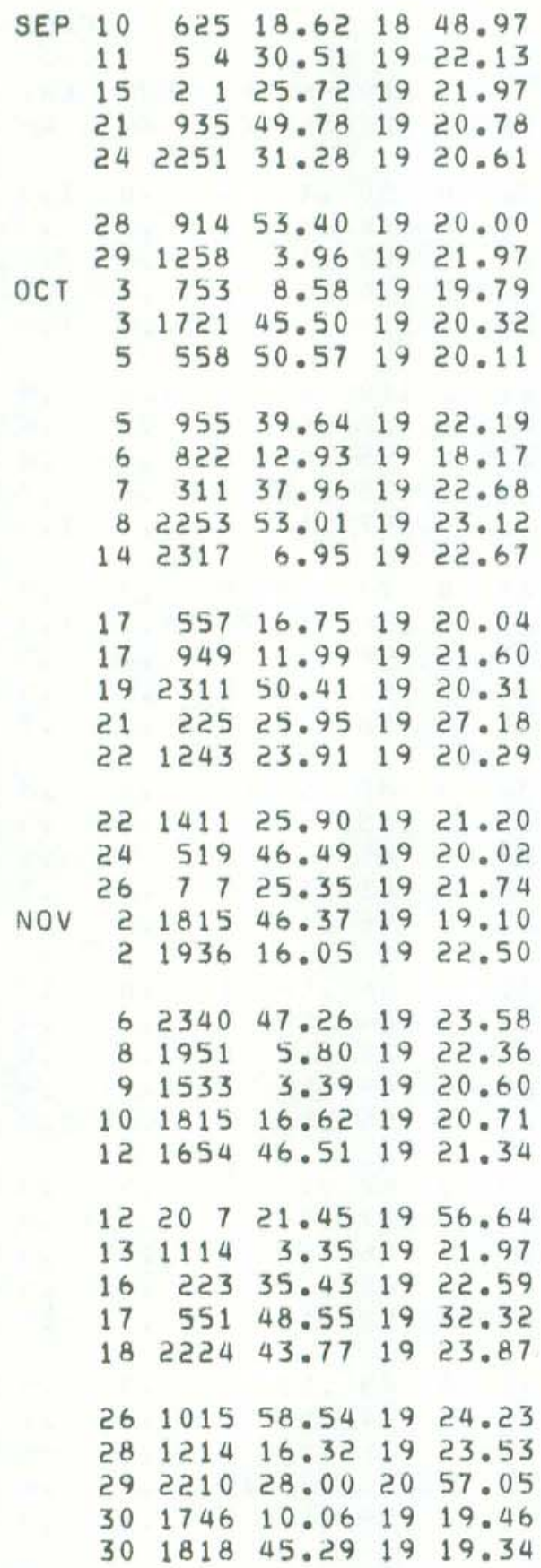

LON W DEG MIN

15513.54

1554.22

$155 \quad 5.05$

1556.54

$155 \quad 6.68$

15513.13

$155 \quad 5.10$

1557.26

$155 \quad 8.02$

1556.59

1554.79

$155 \quad 23.49$

$155 \quad 5.39$

1556.72

1555.12

$155 \quad 8.27$

$155 \quad 6.07$

1556.99

15451.82

15511.42

$155 \quad 3.33$

$155 \quad 8.19$

15515.18

15513.42

1555.86

15525.04

$155 \quad 6.00$

1554.14

15511.73

1552.46

15536.00

$155 \quad 5.07$

1554.70

$155 \quad 14.17$

15516.89

$155 \quad 15.87$

15517.17

15623.23

15511.39

15511.28

15515.23

1558.13

15517.02

$155 \quad 7.61$

15516.86

15510.57

15517.19

15512.04
DEPTH AMP DUR

GAP RMS MIN ERH ERZ KM MAG MAG NR NS DEG SEC DIS KM KM REMK

\begin{tabular}{llllllllll}
36.33 & 3.2 & 4.0 & 28 & 2 & 266 & .14 & 53 & 2.1 & 8.1 \\
\hline$P P L$
\end{tabular} $\begin{array}{lllllllllll}7.61 & 3.6 & 4.0 & 28 & 0 & 94 & .10 & 10 & .6 & .7 & \text { MER }\end{array}$

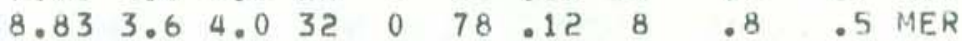
$\begin{array}{llllllllll}9.32 & 3.2 & 3.8 & 29 & 0 & 97 & .11 & 7 & .7 & .5\end{array}$ $\begin{array}{llllllllll}8.95 & 2.9 & 3.7 & 32 & 0 & 99 & .11 & 7 & .7 & .5\end{array}$

$\begin{array}{llllllllllll}10.04 & 3.7 & 4.2 & 34 & 0 & 68 & .10 & 7 & .6 & .2 & \text { UER }\end{array}$

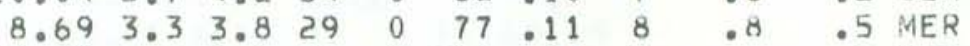
$\begin{array}{llllllllll}9.29 & 3.4 & 3.6 & 31 & 0 & 107 & .11 & 8 & .8 & .5\end{array}$ $9.55 \quad 3.1 \quad 3.6 \quad 31 \quad 0 \quad 84.10 \quad 9 \quad 0.6 \quad .3$ UER $\begin{array}{llllllllll}9.45 & 3.8 & 4.1 & 30 & 0 & 112 & .11 & 7 & .8 & .5\end{array}$

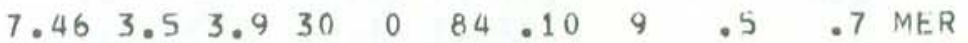
$\begin{array}{lllllllllll}6.08 & 2.6 & 3.6 & 30 & 0 & 109 & .18 & 9 & .9 & 2.0 & \text { SWR }\end{array}$ $\begin{array}{llllllllll}9.26 & 3.5 & 3.9 & 31 & 0 & 79 & .10 & 8 & .6 & .4\end{array}$

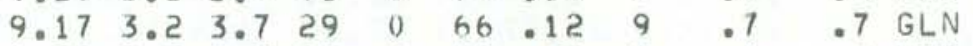

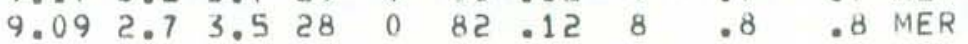

\begin{tabular}{llllllllll}
9.33 & 2.7 & 3.6 & 29 & 0 & 82 & .10 & 9 & .6 & .4 \\
\hline & 4 UER
\end{tabular} $\begin{array}{llllllllll}8.78 & 2.6 & 3.6 & 26 & 0 & 83 & .12 & 7 & .7 & .9\end{array}$ UER

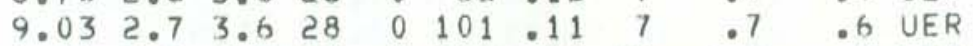
$\begin{array}{lllllllllll}8.48 & 2.9 & 3.5 & 30 & 0 & 267 & .09 & 22 & 1.7 & .5 & \text { LER }\end{array}$ $\begin{array}{lllllllllll}10.38 & 3.4 & 4.1 & 12 & 0 & 104 & .16 & 8 & 1.4 & 2.0 & \text { UER }\end{array}$

\begin{tabular}{|c|c|c|c|c|c|c|c|c|c|c|}
\hline 8.19 & 3.5 & 3.9 & 28 & 0 & 113 & .11 & 11 & .9 & .6 & MER \\
\hline 9.32 & 2.6 & 3.5 & 26 & 0 & 84 & .10 & 9 & .7 & .5 & UER \\
\hline 9. & 3.3 & 3.6 & 27 & 0 & 62 & .11 & 4 & .7 & .5 & KO \\
\hline 1 & 3.7 & 4.0 & 29 & 0 & 73 & .08 & 7 & .5 & .2 & UE \\
\hline .74 & 3.3 & 3.7 & 29 & 0 & 71 & .12 & 7 & .7 & .7 & $A$ \\
\hline
\end{tabular}

$9.75 \quad 3.3 \quad 3.6 \quad 30 \quad 0 \quad 57.12 \quad 9 \quad .6 \quad .5$ UKF $\begin{array}{llllllllll}7.92 & 2.9 & 3.7 & 29 & 0 & 70 & .10 & 7 & .5 & .7\end{array}$ UER $\begin{array}{llllllllll}8.06 & 3.8 & 4.1 & 32 & 0 & 107 & .12 & 9 & .9 & .6\end{array}$ MER $\begin{array}{lllllllllll}9.94 & 3.1 & 3.7 & 29 & 0 & 71 & .10 & 7 & .7 & .3 & 3 \text { UER }\end{array}$ $\begin{array}{llllllllll}7.12 & 3.5 & 4.1 & 31 & 0 & 148 & .13 & 12 & 1.0 & .7\end{array}$

$\begin{array}{llllllllll}11.20 & 3.5 & 26 & 0 & 145 & .14 & 28 & 1.9 & .7 & \mathrm{KOH}\end{array}$ $\begin{array}{lllllllllll}9.18 & 3.7 & 4.1 & 28 & 0 & 77 & .11 & 8 & .7 & .6 & \text { MER }\end{array}$ $9.20 \quad 3.6 \quad 4.1320088 .12 \quad 9 \quad .6 \quad .5$ MER $\begin{array}{lllllllllll}24.99 & 3.7 & 3.7 & 32 & 0 & 66 & .11 & 17 & .7 & 2.1 & \text { NER }\end{array}$

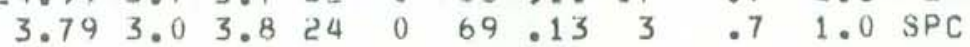

$\begin{array}{lllllllllll}4.49 & 3.1 & 3.8 & 27 & 0 & 46 & .13 & 3 & .6 & .8 & \text { SPC }\end{array}$

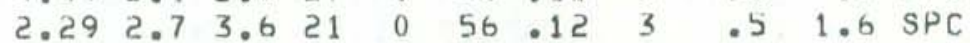
$42.023 .3 \quad 3.7 \quad 3 \quad 2 \quad 360.002589 .7 \quad 41.8$ UIS

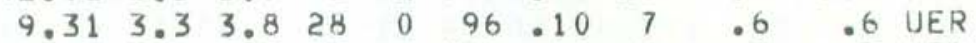
$\begin{array}{lllllllllll}9.65 & 3.0 & 3.5 & 29 & 0 & 100 & .11 & 7 & .7 & .3 & \text { UER }\end{array}$

$9.40 \quad 3.1 \quad 3.8 \quad 32 \quad 0 \quad 61.10 \quad 4 \quad .5 \quad .6 \quad$ KOA $\begin{array}{lllllllllll}8.82 & 3.0 & 3.8 & 29 & 0 & 85 & .11 & 9 & .7 & .5 & \text { UER }\end{array}$

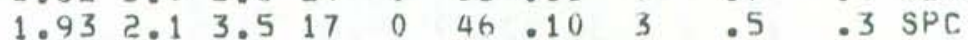
$9.453 .8 \quad 4.129 \quad 0 \quad 74.10 \quad 8 \quad .6 \quad .5$ UER $\begin{array}{lllllllllll}2.99 & 2.7 & 3.6 & 25 & 0 & 47 & .13 & 3 & .6 & 1.2 & \text { SPC }\end{array}$

$\begin{array}{llllllllllll}8.99 & 2.9 & 3.7 & 29 & 0 & 84 & .08 & 7 & .5 & .6 & \text { UER }\end{array}$ $\begin{array}{llllllllll}2.76 & 3.0 & 3.8 & 24 & 0 & 62 & .13 & 3 & .6 & 1.3\end{array}$ SPC

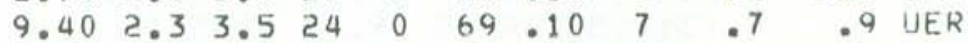




\begin{tabular}{|c|c|c|c|c|c|c|c|c|c|c|c|c|c|c|c|c|c|c|c|}
\hline & & OR. & IGIN T & IME & LA & $T \quad N$ & & $N W$ & DEPTH & AMP & DUR & & & GAP & RMS & MIN & ERH & $E R Z$ & \\
\hline YEAR & MON & DA & HRMN & SEC & $D E G$ & MIN & $D E G$ & MIN & $K M$ & MAG & MAG & NR & NS & DEG & SEC & DIS & $K M$ & $K M$ & REMK \\
\hline 1976 & DEC & 13 & 1726 & 42.07 & 19 & 20.20 & 155 & 7.42 & 9.67 & 3.4 & 4.0 & 29 & 0 & 96 & .12 & 8 & .9 & .4 & UER \\
\hline & & 15 & 440 & 42.94 & 19 & 19.60 & 155 & 11.94 & 9.81 & 2.9 & 3.5 & 33 & 0 & 89 & .12 & 6 & .7 & .3 & UER \\
\hline & & 16 & 2121 & 35.55 & 19 & 25.32 & 155 & 17.03 & .57 & 2.2 & 3.7 & 19 & 0 & 48 & .14 & 4 & .7 & .2 & SPC \\
\hline & & 17 & 339 & 14.61 & 19 & 25.69 & 155 & 16.70 & .80 & 2.9 & 4.1 & 28 & 0 & 48 & .10 & 4 & .3 & .5 & SPC \\
\hline & & 17 & 2031 & 43.29 & 19 & 20.00 & 155 & 6.92 & 9.49 & 3.2 & 3.7 & 31 & 0 & 109 & .09 & 7 & .7 & .5 & UER \\
\hline & & 18 & 41 & .60 & 19 & 20.14 & 155 & 7.01 & 9.39 & 4.8 & 4.9 & 32 & 0 & 104 & .09 & 7 & .6 & .5 & UER \\
\hline & & 26 & 2343 & 9.16 & 19 & 47.76 & 155 & 58.54 & 7.82 & 2.9 & 3.5 & 19 & 0 & 220 & .19 & 42 & 3.8 & 1.6 & KUN \\
\hline & & 27 & 415 & 20.58 & 19 & 23.61 & 155 & 14.75 & 5.22 & 3.3 & 4.0 & 30 & 0 & 44 & .12 & 3 & .4 & .6 & GLN \\
\hline & & 27 & 624 & 27.46 & 19 & 19.45 & 155 & 16.53 & 9.63 & $3 \cdot 1$ & 3.5 & 32. & 0 & 97 & .10 & 6 & .6 & .5 & KOA \\
\hline & & 28 & 1517 & 36.61 & 19 & 23.88 & 155 & 16.86 & 1.94 & 2.8 & 3.5 & 19 & 0 & 70 & .09 & 3 & .4 & .2 & SPC \\
\hline & & 28 & 1937 & 4.91 & 19 & 19.21 & 155 & 12.16 & 9.77 & 3.2 & 3.8 & 29 & 0 & 95 & .11 & 7 & .7 & .3 & UER \\
\hline & & 29 & 811 & 17.14 & 19 & 19.33 & 155 & 12.41 & 8.77 & 2.6 & 3.6 & 27 & 0 & 88 & .12 & 7 & .7 & 1.0 & UER \\
\hline & & 29 & 1644 & 25.63 & 19 & 19.84 & 155 & 11.26 & 9.15 & 2.9 & 3.5 & 24 & 0 & 88 & .09 & 6 & .5 & .7 & UER \\
\hline & & 29 & 1926 & 26.92 & 19 & 23.48 & 155 & 14.49 & 4.59 & 3.0 & 3.8 & 24 & 0 & 54 & .10 & 3 & .5 & .8 & GLN \\
\hline & & 30 & 047 & 41.50 & 18 & 27.18 & 155 & 20.11 & 11.66 & 3.8 & 4.1 & 9 & 0 & 316 & .07 & 92 & 25.3 & .0 & DIS \\
\hline & & 30 & 419 & 51.59 & 19 & 19.52 & 155 & 15.93 & 9.58 & 3.0 & 3.8 & 30 & 0 & 91 & .08 & 6 & .4 & .3 & KOA \\
\hline
\end{tabular}




\section{TILT INSTRUMENTATION}

In addition to the seismic network, a network of spirit-level tilt stations (dry), borehole tiltmeters, and water-tube (wet) tilt stations is maintained. The network is located on the summits and flanks of Kilauea and Mauna Loa Volcanoes. In December 1976 the tilt network consisted of:

73 spirit level tilt stations (dry)

8 borehole tiltmeters

10 water-tube tilt stations (wet)

1 continuous recording Ideal-Arrowsmith tiltmeter

Dry and wet tilt stations are generally occupied at irregular intervals. Critical stations are measured more frequently than the entire network. Digital borehole tiltmeters data are telemetered by VHF radio and recorded at the observatory. An Ideal-Arrowsmith mercury-pool capacitor-type tiltmeter with a $1 \mathrm{~m}$ base is located at the Uwekahuna vault, and the analog signal is recorded at the observatory. 
Tilting of the ground around the summit of Kilauea is monitored daily by a short-base water-tube tiltmeter in Uwekahuna Vault, and at irregular intervals it is measured on a regional scale by means of a network of field tilt-bases and a portable water-tube tiltmeter. The attitude of the ground surface at each tilt-base is reported in terms of north-south and east-west tilt coordinates. Both coordinates at each station were arbitrarily set equal to 500 when measurements at that station were begun. Increasing tilt coordinates correspond to northward and eastward tilting of the earth's surface; that is, to a relative subsidence toward the north and east. A oneunit change in coordinate corresponds to a tilting of 1 microradian (1 $\mathrm{mm}$ per $\mathrm{km}$ ) in the direction indicated.

Location of and essential data on each tiltmeter station are listed in table 8 .

Table 7.--Tilt Coordinates at Uwekahuna

\begin{tabular}{|c|c|c|}
\hline Date (1976) & $\mathrm{N}-\mathrm{S}$ & $\mathrm{E}-\mathrm{W}$ \\
\hline $\begin{array}{r}\text { Jan } 4 \\
11 \\
18 \\
25\end{array}$ & $\begin{array}{l}573 \\
569 \\
567 \\
564\end{array}$ & $\begin{array}{l}402 \\
401 \\
399 \\
401\end{array}$ \\
\hline $\begin{array}{rr}\text { Feb } \quad 1 \\
8 \\
15 \\
22 \\
29\end{array}$ & $\begin{array}{l}562 \\
560 \\
569 \\
558 \\
575\end{array}$ & $\begin{array}{l}401 \\
399 \\
398 \\
398 \\
396\end{array}$ \\
\hline $\begin{array}{r}\quad 7 \\
14 \\
21 \\
28\end{array}$ & $\begin{array}{l}556 \\
557 \\
556 \\
556\end{array}$ & $\begin{array}{l}396 \\
392 \\
393 \\
391\end{array}$ \\
\hline $\begin{array}{rr}\text { Apr } & 4 \\
11 \\
18 \\
25\end{array}$ & $\begin{array}{l}556 \\
557 \\
558 \\
558\end{array}$ & $\begin{array}{l}389 \\
388 \\
390 \\
387\end{array}$ \\
\hline $\begin{array}{r}2 \\
9 \\
16 \\
23 \\
30\end{array}$ & $\begin{array}{l}558 \\
559 \\
560 \\
561 \\
561\end{array}$ & $\begin{array}{l}388 \\
388 \\
390 \\
387 \\
385\end{array}$ \\
\hline $\begin{array}{r}\text { Jun } 6 \\
13 \\
20\end{array}$ & $\begin{array}{l}562 \\
561 \\
560\end{array}$ & $\begin{array}{l}384 \\
382 \\
382\end{array}$ \\
\hline 27 & $\begin{array}{c}559 \\
\mathbf{6 1}\end{array}$ & 383 \\
\hline
\end{tabular}


Table 7.--Tilt Coordinates at Uwekahuna (Continued)

\begin{tabular}{|rll|}
\hline Date $(1976)$ & N-S & E-W \\
\hline JuI 4 & 560 & 380 \\
11 & 559 & 382 \\
18 & 558 & 387 \\
25 & 558 & 385 \\
Aug 1 & 558 & 380 \\
8 & 558 & 382 \\
15 & 558 & 379 \\
22 & 556 & 377 \\
29 & 582 & 389 \\
Sep 5 & 549 & 399 \\
12 & 545 & 408 \\
19 & 540 & 412 \\
26 & 536 & 412 \\
Oct 3 & 535 & 410 \\
10 & 534 & 407 \\
17 & 536 & 402 \\
24 & 537 & 397 \\
31 & 538 & 393 \\
7 & 539 & 388 \\
14 & 539 & 386 \\
21 & 541 & 382 \\
28 & 541 & 381 \\
Dec & 543 & 379 \\
12 & 543 & 377 \\
19 & 543 & 369 \\
26 & 544 &
\end{tabular}


Table 8.--U.S. Geological Survey water-tube tiltmeter stations in Hawaii

\begin{tabular}{|c|c|c|c|c|c|c|}
\hline \multirow{2}{*}{ Station } & \multirow{2}{*}{ Symbol } & \multicolumn{2}{|c|}{ Location } & \multirow{2}{*}{$\begin{array}{c}\text { Frequency } \\
\text { of } \\
\text { reading }\end{array}$} & \multirow{2}{*}{$\begin{array}{c}\text { Base } \\
\text { length } \\
\text { M }\end{array}$} & \multirow{2}{*}{ Description } \\
\hline & & $\begin{array}{l}\text { Lat. N. } \\
\text { Deg. Min. }\end{array}$ & $\begin{array}{l}\text { Long. W. } \\
\text { Deg. Min. }\end{array}$ & & & \\
\hline Tree Molds & TM & $19-26.3$ & $155-17.3$ & & 50.79 & NS. and EW. \\
\hline Sand Sp1t & SS & $19-24.1$ & $155-16.8$ & & 25.40 & $\begin{array}{c}\text { Equilateral } \\
\text { triangle. }\end{array}$ \\
\hline Keamoku & Kea & $19-25.1$ & $155-19.0$ & & 47.55 & do \\
\hline Ahua & & & & & & \\
\hline Kamokukolau & Kam & $19-22.7$ & $155-16.6$ & & 50.79 & do \\
\hline K1puka Nene & $\mathrm{KN}$ & $19-19.4$ & $155-16.7$ & & 47.73 & do \\
\hline H1lina Pal1 & HP & $19-18.2$ & $155-18.6$ & & 47.73 & do \\
\hline $\begin{array}{c}\text { Kapapala } \\
\text { Ranch }\end{array}$ & Kap & $19-20.5$ & $155-23.8$ & & 50.79 & do \\
\hline Mehana & M & $19-26.2$ & $155-14.3$ & & 25.00 & do \\
\hline Uwekahuna & U & $19-25.5$ & $155-17.4$ & & 50.79 & do \\
\hline $\begin{array}{c}\text { Uwekahuna } \\
\text { Vault }\end{array}$ & & $19-25.4$ & $155-17.6$ & Da1ly & 3.48 & NS. and EW. \\
\hline
\end{tabular}


Table 9. Tilt coordinates and changes at bases around Kilauea caldera. (See fig.10)

\begin{tabular}{|c|c|c|c|c|c|c|}
\hline \multirow[b]{2}{*}{ Uwekahuna (U on fig.10) } & \multirow{2}{*}{$\begin{array}{c}\begin{array}{c}\text { Date } \\
(\text { i } 976)\end{array} \\
11 \text { May }\end{array}$} & \multicolumn{2}{|c|}{${ }_{\mathrm{N}-\mathrm{S}}^{\text {Tilt coordinates }}$} & \multicolumn{2}{|c|}{$\begin{array}{c}\text { Rate }\left(10^{-6} \mathrm{rad} / \mathrm{mo}\right) \\
\text { and direction of } \\
\text { tilting since } \\
\text { last reading }\end{array}$} & $\begin{array}{c}\text { Date of last } \\
\text { reading } \\
(1975)\end{array}$ \\
\hline & & 597.6 & 346.8 & 8.96 & $1773.7^{\circ} \mathrm{W}$ & $19 \mathrm{Dec}$ \\
\hline Tree Molds (TM) & 11 May & $494 \cdot 7$ & 513.3 & 1.50 & $\mathrm{~N} 47.7^{\circ} \mathrm{W}$ & $16 \mathrm{Dec}$ \\
\hline Sand Spit (SS) & $14 \mathrm{Apr}$ & 805.8 & 855.2 & 12.69 & $\mathrm{~S} 44.0^{\circ} \mathrm{E}$ & $18 \mathrm{Dec}$ \\
\hline Keamoku (Kea) & $13 \mathrm{Apr}$ & 576.5 & 496.6 & 7.49 & $\mathrm{~S} 33.9^{\circ} \mathrm{E}$ & $17 \mathrm{Dec}$ \\
\hline Ahua Kamokukolau (Kam) & 14 Apr & 703.6 & $554 \cdot 9$ & 12.14 & $\mathrm{~N} 6.8^{\circ} \mathrm{W}$ & $18 \mathrm{Dec}$ \\
\hline Kipuka Nene (KN) & $21 \mathrm{Apr}$ & 156.2 & 576.7 & 4.65 & $\mathrm{~N} 41.0^{\circ} \mathrm{W}$ & $15 \mathrm{Dec}$ \\
\hline Hilina Pali (HP) & $15 \mathrm{Apr}$ & 309.7 & 550.8 & 6.80 & $\mathrm{~N} 30.9^{\circ} \mathrm{W}$ & $15 \mathrm{Dec}$ \\
\hline Kapapala (Kap) & $13 \mathrm{Apr}$ & 513.9 & 517.4 & 3.10 & $.569 .0^{\circ} \mathrm{E}$ & $17 \mathrm{Dec}$ \\
\hline Mehana (M) & 12 Apr & 548.8 & 560.2 & 3.39 & $57.8^{\circ} \mathrm{H}$ & $16 \mathrm{Dec}$ \\
\hline
\end{tabular}




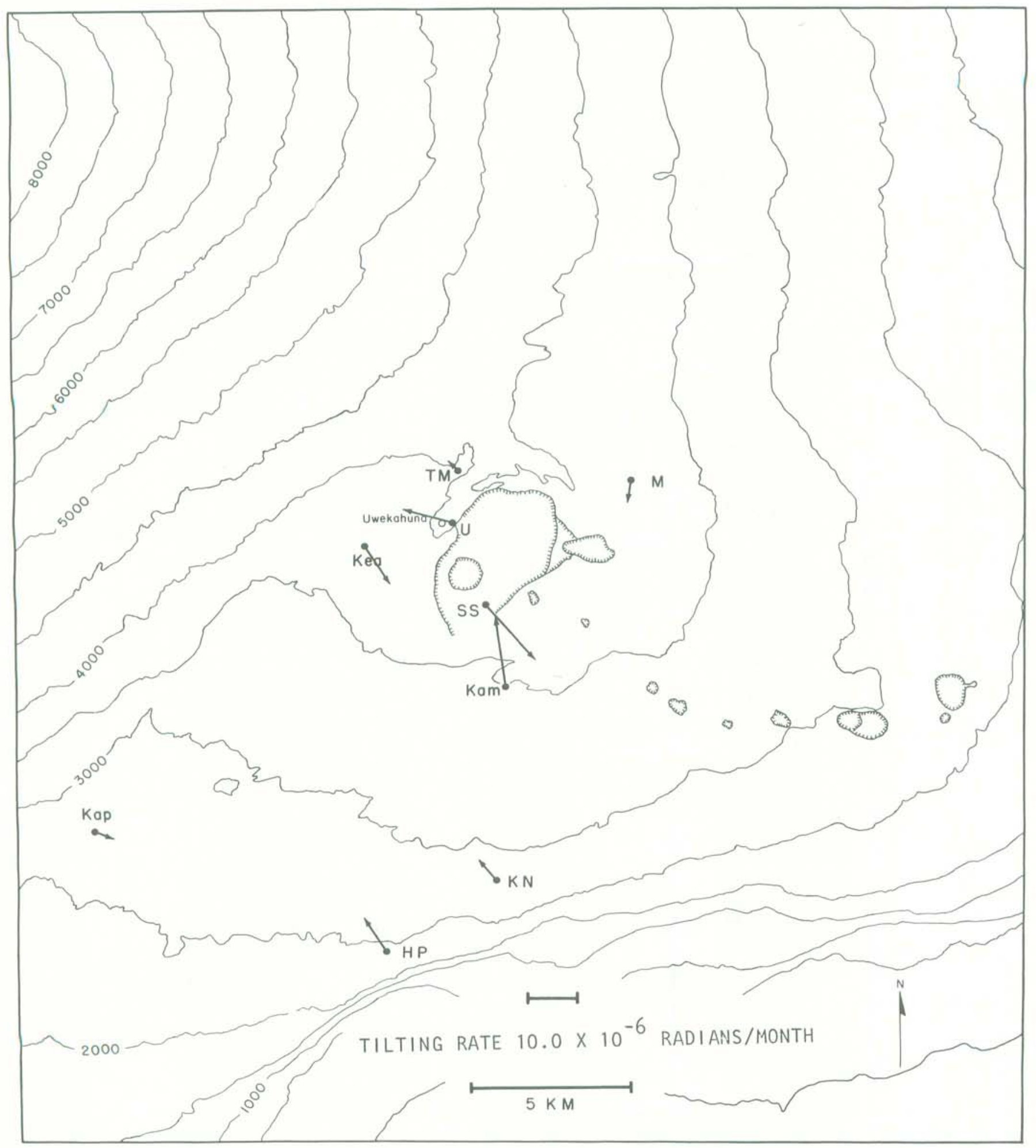

Figure 10 December 1975 to April 1976 tilting of the ground around Kilauea Caldera. The vector depicting tilt at a given tilt base points in the direction of maximum relative subsidence, and its length is proportional to the rate of tilting during the measurement interval. Closed circles represent field tilt bases; open circles, short-base watertube tiltmeters. See Table 8 for explanation of abbreviations. 
Table 10. Tilt coordinates and changes at bases around Kilauea caldera. (See fig.11)

\begin{tabular}{|c|c|c|c|c|c|c|}
\hline \multirow{2}{*}{$\begin{array}{c}\text { Tilt base } \\
\text { Uwekahuna (U on } \mathrm{fig} \cdot 11 \text { ) }\end{array}$} & \multirow[t]{2}{*}{$\begin{array}{c}\text { Date } \\
(1976)\end{array}$} & \multicolumn{2}{|c|}{$\begin{array}{c}\text { Tilt coordinates } \\
\mathrm{N}-\mathrm{S} \\
\mathrm{E}-\mathrm{W}\end{array}$} & \multicolumn{2}{|c|}{$\begin{array}{l}\text { Rate }\left(10^{-6} \mathrm{rad} / \mathrm{mo}\right) \\
\text { and direction of } \\
\text { tilting since } \\
\text { last reading }\end{array}$} & \multirow{2}{*}{$\begin{array}{c}\text { Date of last } \\
\text { reading } \\
(1976)\end{array}$} \\
\hline & & & Not Oc & This & $\operatorname{pch}$ & \\
\hline Tree Molds (TM) & & & Not Oc & d This & $\mathrm{sch}$ & \\
\hline Sand Spit (SS) & & & Not $\mathrm{Oc}$ & dhis & $\operatorname{cch}$ & \\
\hline Keamoku (Kea) & & & Not $\mathrm{Oc}$ & This & $\mathrm{ch}$ & \\
\hline Ahua Kamokukolau (Kam) & & & Not $\mathrm{Oc}$ & dhis & $\operatorname{ch}$ & \\
\hline Kipuka Nene (KN) & 28 Sep & 164.6 & 564.0 & 2.82 & $1 \mathrm{~N} 57.8^{\circ} \mathrm{W}$ & $21 \mathrm{Apr}$ \\
\hline Hilina Pali (HP) & & & Not $\mathrm{Oc}$ & d This $\mathrm{E}$ & ch & \\
\hline Kapapala (Kap) & $27 \mathrm{Sep}$ & $514 \cdot 7$ & 522.1 & 0.85 & $1180.0^{\circ} \mathrm{E}$ & $13 \mathrm{Apr}$ \\
\hline Mehana (M) & $27 \mathrm{Sep}$ & 535.9 & 558.6 & 2.32 & $37.2^{\circ} \mathrm{W}$ & 12 Apr \\
\hline
\end{tabular}




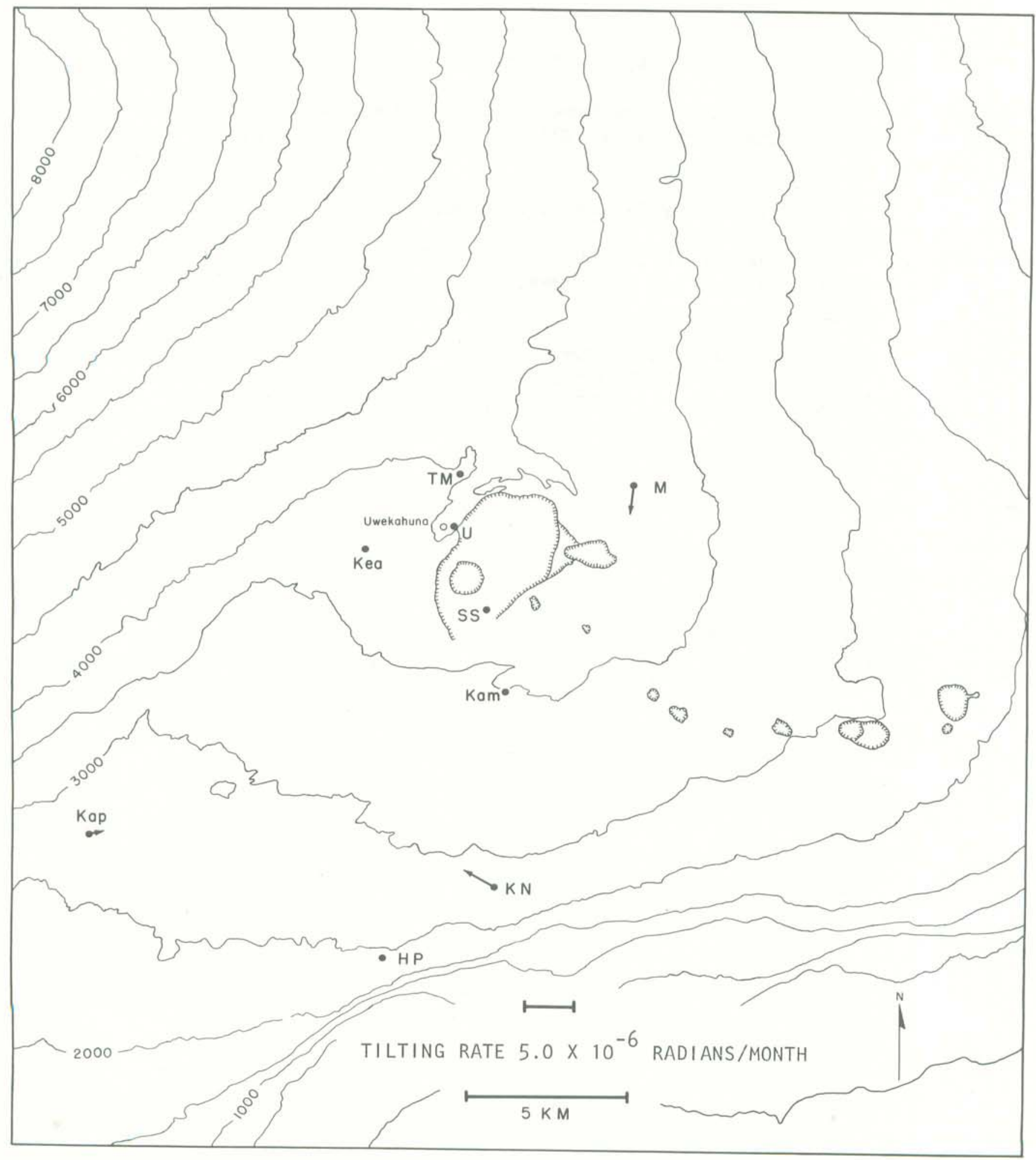

Figure 11 April to September 1976 tilting of the ground around Kilauea Caldera. 


\section{REFERENCES CITED}

Crosson, R. S., 1976, Velocity structure below the Island of Hawaii from earthquake modelling, (abstract), EOS Trans. Am. Geoph. Union, 57, 961.

Koyanagi, R. Y., Stevenson, P., Endo, E. T., and Okamura, A. T., Hawaiian Volcano Observatory Summary 74, January to December 1974.

Lockwood, J. P., Koyanagi, R. Y., Tilling, R. I., Holcomb, R. T., and Peterson, D. W., 1976, Mauna Loa threatening: Geotimes, v. 21, no. 6, p. $12-15$.

Tilling, R. I., Koyanagi, R. Y., Lipman, P. W., Lockwood, J. P., Moore, J. G., and Swanson, D. A., 1976, Earthquakes and related catastrophic events, Island of Hawaii, November 29, 1975 -- a preliminary report: U.S. Geol. Survey Circ. 740,33 p. 